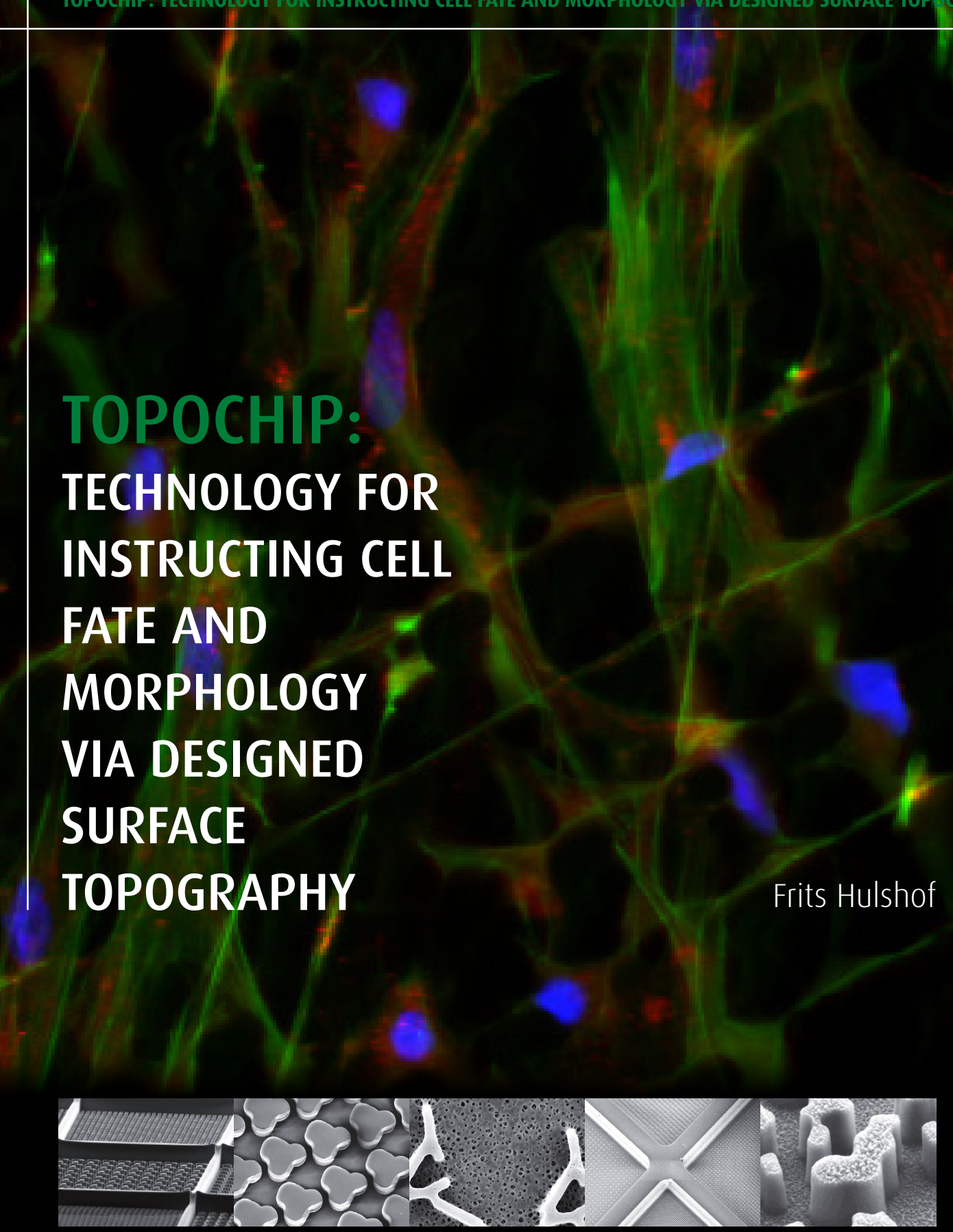




\section{TOPOCHIP: TECHNOLOGY FOR INSTRUCTING CELL FATE}

AND MORPHOLOGY VIA DESIGNED SURFACE TOPOGRAPHY 
The research described in this thesis was financially supported by the NanoNext NL initiative, cluster $6 \mathrm{C}$

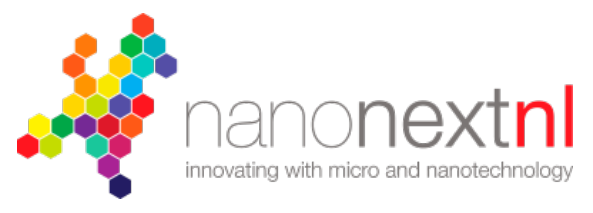

The printing of this thesis was sponsored by:

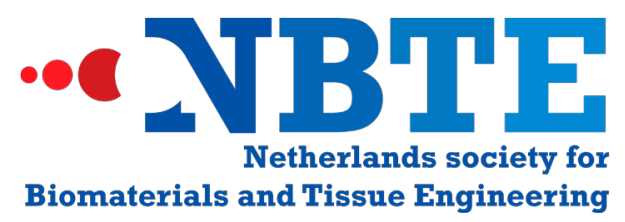

Topochip: Technology for Instructing Cell Fate and Morphology via Designed Surface Topography

Frits Hulshof

PhD Thesis, with references and summaries in English and Dutch

University of Twente, Enschede, The Netherlands

Mei 2016

Cover design and thesis layout by Ties Horneman

Printed by Gildeprint, Enschede, The Netherlands 


\title{
TOPOCHIP: TECHNOLOGY FOR INSTRUCTING CELL FATE AND MORPHOLOGY VIA DESIGNED SURFACE TOPOGRAPHY
}

\author{
PROEFSCHRIFT
}

ter verkrijging van

de graad van doctor aan de Universiteit Twente,

op gezag van de rector magnificus,

prof H. Brinksma,

volgens besluit van het College voor promoties

in het openbar te verdedigen op

donderdag 26 mei 201612.45

door

Godefridus Fransciscus Bernardus Hulshof

geboren op 13 januari 1986

te Enschede 
Dit proefschrift is goedgekeurd door de promotoren:

Prof. Dr. J. de Boer

Prof. Dr. D. Stamatialis

(c) 2016 Frits Hulshof

ISBN: 978-90-365-4122-0

DOI: $10.3990 / 1.978903654120$ 


\section{THESIS COMMITTEE}

Chairman

Promoters

Assistant promoter

Members
Prof. Dr. Ir. J.W.M. Hilgenkamp

Prof. Dr. D. Stamatialis

Prof. Dr. J. de Boer

Prof. Dr. C.A. van Blitterswijk

Prof. Dr. D.W. Grijpma

Prof. Dr. Ir. P. Jonkheijm

Prof. Dr. R. Masereeuw

Prof. Dr. M.J.T. Reinders

Dr. B.J. Papenburg 


\section{TABLE OF CONTENTS}

\section{Chapter 1}

General Introduction

\section{Chapter 2}

High-content imaging 16

\section{Chapter 3}

Mining for osteogenic surface topographies:

In silico design to in vivo osseo-integration

\section{Chapter 4}

Designed polystyrene microtopographies inhibit

osteogenic and adipogenic differentiation of hMSCs.

\section{Chapter 5}

New insights into the effects of biomaterial chemistry

and topography on morphology and function of

kidney epithelial cells

\section{Chapter 6}

High-throughput screening of cell instructive effects

by designed nanoscale topography

\section{Chapter 7}

Discussion and outlook

\section{Appendix 1}

Micropatterning polystyrene: a high-quality method to obtain large area and high density topographies.

\section{Appendix 2}

Pluripotency inducing topography for tissue culture:

a Technology assessment on the translation of designed topography towards a cell-culture product. 
CHAPTER 1

\section{GENERAL INTRODUCTION}




\section{Modern biomaterials}

A biomaterial is any matter, surface, or construct that interacts with living systems. Biomaterials are used to make all sorts of medical applications, from relatively simple things such as band-aids and stitching thread to complex devices such as artificial joints and heart-valves. Many different materials can be biomaterials such as natural and synthetic polymers, metals, ceramics and glasses. Historically, for biomedical design, the first objective was biocompatibility of the material or the prevention of rejection by the host body. Throughout the years the properties of biomaterials have had to meet higher demands. Successful integration into the host body became expected. Next, some biomaterials should only perform a temporary function in the host body, slowly degrading while the native tissue rebuilds. These days biomaterials are developed with the objective to aid healing of the damaged tissue by instructing repair with mechanical and biological cues.

Biomaterials are also used in vitro as cell culture substrates in life sciences. Biological sciences use relatively artificial materials for their in vitro work such as high molecular weight polystyrene and glass. These materials have been used for decades due to their low cost and good optical properties for microscopy and there is obviously reluctance to switch from a working system. Unfortunately most cells lose functional properties ex vivo. By advancing cell culture biomaterials it should be possible to keep cells functional ex vivo for extended amounts of time. For example, maintaining the pluripotency of stem cells in vitro is the objective of many studies (1-3).

\section{Biomaterial-cell interactions}

To improve tissue engineering products, implants and tissue culture substrates it is vital to understand and improve cell-biomaterial interactions. These interactions are governed by material parameters such as material stiffness (4), surface chemistry $(5)$, degradability $(6,7)$ and surface topography. Inspiration for these parameters are the mechanical and chemical properties from the native tissues from which the cells are derived. For example, stem cells differentiate towards cell types on substrates that have a corresponding stiffness to the native tissue of the differentiated cells (8).

\section{Surface topography}

In this thesis we focus on the effects of surface topography on cells and tissues. Already, clinical efficacy of applying topography to improve implants is established. For example, titanium bone implants are sand-blasted and acid etched to create micro and nano roughness to improve implant performance (9). Even though there is clear evidence of clinical effects, the underlying bio-physical mechanisms and molecular pathways are not evident. Rational, controlled design of surface topography, made possible by microfabrication techniques, enables more systematic investigation of topographical effects. In the last decade there have been several studies showing the various effects of designed topographies, fabricated in different scales in cell differentiation and morphology. Dalby et. al. showed that a moderately disordered spacing of pits with a diameter of $120 \mathrm{~nm}$ induced an osteogenic response of human mesenchymal stromal cells (hMSC), which did not occur when the same pits were spaced in a ordered or completely random fashion (10). The disordered spacing of the pits induced formation of bone nodules and a small upregulation of osteogenic genes. Additionally, culture on the topography led to the activation of molecular pathways of which some are also activated when the cells are exposed to dexamethasone, which is a osteogenic supplement. Li et, al. showed that microgrooves with a depth of $3 \mu \mathrm{m}$ and a width of $10 \mu \mathrm{m}$ are 
able to improve the reprogramming efficiency of fibroblasts to induced pluriotent stem cells, compared to planar surfaces (11). The increased efficiency was caused by a decrease of histone deacetylase activity and an increase in the WDR5 histone methyltransferase subunit expression. The same study showed that when the elongated cell morphology with aligned actin fibers, visible on the microtopographies was replicated in fibroblasts cultured on aligned sub-micrometer sized elect spun fibers, it resulted in similar increase of reprogramming efficiency. This is a clear example of correlation between cell morphology and downstream effect of a molecular pathway, which shows that cells can be mechanically regulated by topographies of varying scales.

\section{High-throughput screening}

Even though the controlled design of topographies has greatly benefitted the studies for cell-topography interactions, this approach has not yet improved our knowledge of the biophysical mechanisms and downstream pathways sufficiently to apply topographies to improve biomedical devices and tissue culture substrates. To identify topography that benefit certain biological processes, in a cost and time efficient manner, we require high-throughput screening (HTS) of topographies. HTS for surface topographies with beneficial biological instructive effect is very similar to screening for other biomaterial properties $(1,12$, 13). Figure 1 shows a scheme of the components of a high-throughput biomaterial image-based screening experiment. Such an experiment is usually initiated to tailor biomaterial properties to improve response of cells and tissues. Before a screening experiment can be started two things have to be established: a suitable, miniaturized materials library and a robust biological assay.

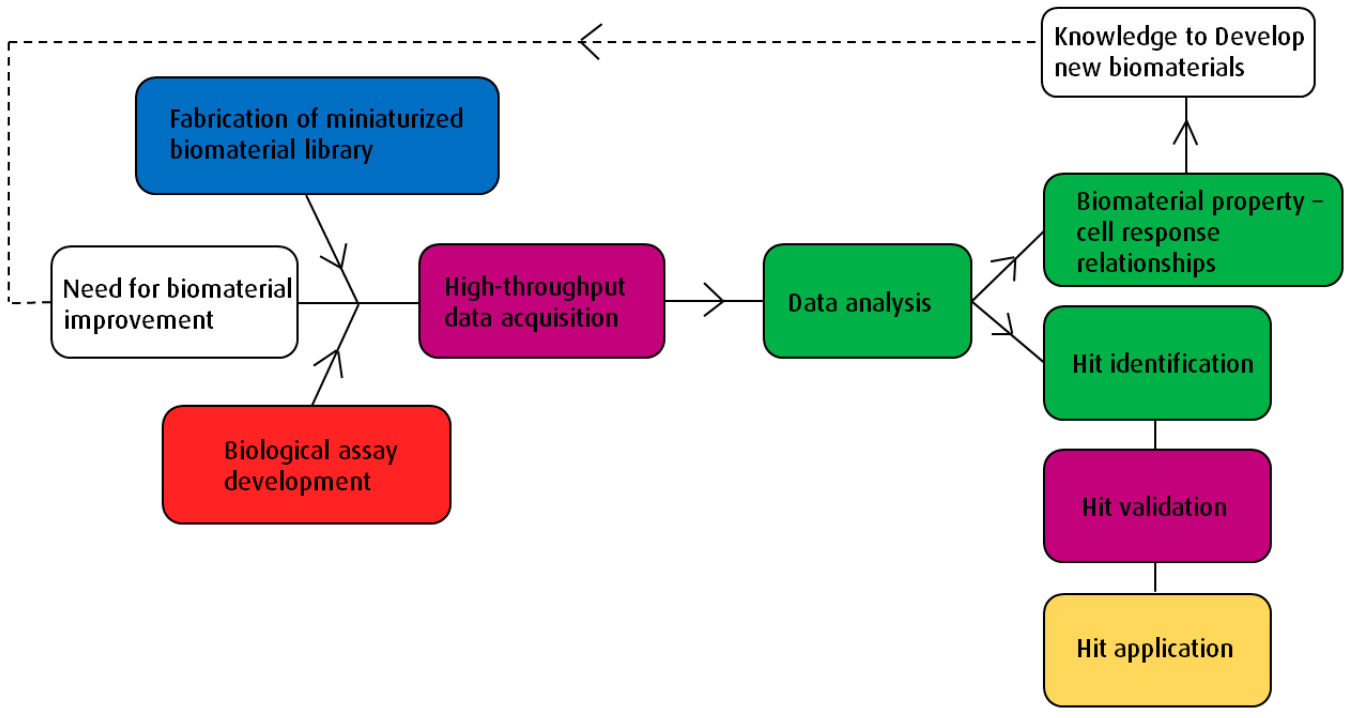

Figure 1: Experimental workflow of biomaterial high-throughput screening for cell responses.

The creation of a screening platform with designed surface topographies requires microfabrication techniques such as photolithography which originate from the micro-electronics industry. Photolithography is the transfer of geometric patterns into a photosensitive material via selective exposure to light. The resoIution of photolithography is limited by the wavelength used, which in practice means several hundreds 
of nanometers in contact printing used in research. For improved resolutions, down to the scale of tens of nanometers, beam writing techniques such as ion beam or electron beam lithography can be used. Unfortunately, beam writing techniques often suffer from long writing times which severely limits the size of the writing area. To create topographical features on polymers suitable for biological experiments, hot embossing (14) is often used. In the hot embossing process a mould, which is usually created by photolithography, is pressed into a heated polymer film to create an imprint. Most thermoplasts can be used for hot embossing. The resolution of hot embossing is mainly limited by the resolution of the mould. The main difficulty in hot embossing is the successful demoulding without damaging both the mould and thermoplastic film, which can be difficult with hard and brittle polymers such as PS. In our work we use specialized Ormostamp ${ }^{\circledR}$ moulds to emboss PS (Appendix 1).

The second requirement for HTS of biomaterials is a biological assay with a robust readout. For this a suitable cell model is required, which should mimic the cells that interact with the eventual application such as a endothelial cells if one aims to improve stent materials. Both primary cells and cell lines can be viable. Even though a primary cell type is less artificial compared to the in vivo situation, it is often in limited supply and can also be very heterogeneous which adds additional 'noise' to the dataset generated by the screening experiment. Alternatively, a cell line can be used, which is more artificial but has the advantages of being cheaper to maintain and consisting of a more homogenous population. Moreover, cell lines are more suitable to transfect with transgenes that carry reporter constructs to create reporter cell lines. Reporter cell-lines greatly improve the data quality of HTS as there is no need for staining with labeled antibodies. The readout usually consists of the staining of an expressed protein with a fluorescently labeled antibody to show the change of protein level and/or location in the cells which can be observed by microscopy.

With a biomaterial library and a robust sensitive assay, the screening experiment can be performed with suitable HTS imaging system to create the raw images. This imaging process and the basics of image analysis are covered in Chapter 2.

The dataset generated by HTS can be queried in various ways. Firstly, the substrates with the best biological performance can be identified as 'hits'. These hit substrates should be validated to remove false positives that occur in HTS. Validation experiments are preferably performed with scaled up versions of the hit substrates. For example, in the case of variation in surface chemistry, the substrates may have to be synthesized on a larger scale. For surface topographies the structure has to be applied to a larger surface area. True positive hits are candidates for application in medical devices or tissue culture products. The dataset can also be used to infer relationships between surface chemistry or surface feature shape parameters and biological readouts such as cell morphology and marker expression. This information can provide important clues to uncover the underlying biophysical mechanisms which can aid in the development of future biomaterials.

There are several examples in literature in which the authors take advantage of the vast design space provided by microfabrication technologies to screen large numbers of topographies for cell response. Kolind et. al. fabricated tantalum coated silicon chips which contained 169 distinct surfaces with topographies that concisted of $2.4 \mu \mathrm{m}$ high squares and circles with various spacings and 'sharkfin like' topographies from rectangles with various spacings (15). The systematic study revealed that larger spacing of the microstructures increased proliferation. The microtopographies also affected the morphology and number of focal adhesions. Li et. al. created a PDMS chip with microscale feature ranging between 2 and $15 \mu \mathrm{m}$ (16). The 71 different features consisted of anisotropic shapes (linear, circular or angular gratings, triangles and rectangles) or isotropic shapes (dots, grids and squares). The Chip was used to investigate the effect 
microtopographies on neuronal outgrowth. The results from their screening experiment showed that the anisotropic topographies promote axonal extension relative to the isotropic topographies.

To perform HTS of microtopographies our group developed the Topochip. The Topochip contains 2176 unique topographical features in duplicate, which are randomly selected from an in silico library of more than 150 million topographies, generated by combining the primitive shapes: triangle, rectangle and circle. By combining the primitives in varying numbers and sizes, any conceivable shape can be created. A proof of principle screening experiment with Topochips fabricated from poly lactic acid showed that topographies were able to induce hMSC proliferation and osteogenic marker expression (17). Furthermore the cellular responses could be correlated to the design parameters.

In this thesis we use the state of the art Topochip HTS platform to investigate bioactive topographies on materials which are similar to the intended (biomedical) application. The first aim of the thesis is to identify and further study microtopographies for the improvement of several distinct applications: an orthopedic bone implant, a membrane for a bio-artificial kidney device and tissue culture plastic ware. Because topographies are also able to instruct cells at the nanoscale. The second aim of this thesis is to extend the screening of microtopographies for cell response to nanotopographies with the development of the Nano-Topochip.

\section{Outline of thesis.}

Chapter 2 will discuss high-throughput, high-content imaging. A large part of the work in this thesis is based on the Topochip HTS platform, which relies heavily on the imaging techniques and image analysis methods described in this chapter. This chapter is a clear tutorial and highlights the many possibilities, advantages and disadvantages that come with high-throughput imaging.

The first experimental chapter (Chapter 3) will demonstrate how the titanium coated Topochip is used to identify osteogenic topographies. The osteogenic topographies are evaluated by studying their effect on osteogenic differentiation of hMSC in vitro and on the performance of titanium bone implants in vivo. Furthermore we will use the screening system for the inference of relationships between topographical feature shapes, cell morphology and cell differentiation, analogous to identification of drug identification by computational chemistry.

The second experimental chapter (Chapter 4) will evaluate the effect of the osteogenic topographies identified in Chapter 3, fabricated in polystyrene (PS), on hMSC osteogenesis and adipogenesis. Several phenotypic assays will be used to characterize the instructive effect of the microtopographies. Finally the role of surface chemistry in the cell-instructive effect of topographies will be investigated by comparing the results obtained with the PS topographies to the data from titanium topographies.

The third experimental chapter (Chapter 5) will cover a study on the effects of surface topography on kidney epithelial cell monolayers with the aim of improving membranes of bio-artificial kidney devices. For this, several bio-active topographies will be fabricated on porous PolyetherSulphone-Polyvinylpyrrolidone (PES-PVP) membranes by phase separation micro-moulding. Cell adhesive coatings will be tested which will be required to culture cells on the PES-PVP membranes which normally inhibit cell adhesion due to their resistance to biofouling. The effect of surface topography the cell monolayer formation will be evaluated by imaging kidney epithelial cells cultured on the membranes. 
The fourth and final experimental chapter (Chapter 6) will present the fabrication of the NanoTopochip which has topographical features of lateral dimensions between $100 \mathrm{~nm}$ and $2 \mu \mathrm{m}$, which is more than 50 times smaller than the micro-Topochip. For the fabrication of the nano features on a large area of $4 \mathrm{~cm}^{2}$, deep UV lithography, normally only used in chip industry, is applied. The effect the nanotopographies on u2OS cell morphology will be investigated in a high-throughput screening experiment and compared to the micro-Topochip.

Chapter 7 will present a general discussion of the thesis findings and an outlook of the work.

A technology assessment on concept of applying microtopographies to tissue culture plastics will be performed in Appendix 2. 


\section{Literature}

1. Gobaa S, Hoehnel S, Roccio M, Negro A, Kobel S, Lutolf MP. Artificial niche microarrays for probing single stem cell fate in high throughput. Nature methods. 2011 Nov;8(11):949-55. PubMed PMID: 21983923. Epub 2011/10/11. eng.

2. Chen W, Shao Y, Li X, Zhao G, Fu J. Nanotopographical Surfaces for Stem Cell Fate Control: Engineering Mechanobiology from the Bottom. Nano today. 2014 Dec 1;9 (6):759-84. PubMed PMID: 25883674. Pubmed Central PMCID: PMC4394389. Epub 2015/04/18. Eng.

3. Li Y, Chu JS, Kurpinski K, Li X, Bautista DM, Yang L, et al. Biophysical regulation of histone acetylation in mesenchymal stem cells. Biophysical journal. 2011 Apr 20; 100(8):1902-9. PubMed PMID: 21504726. Pubmed Central PMCID: PMC3077706. Epub 2011/04/21. eng.

4. Engler AJ, Sen S, Sweeney HL, Discher DE. Matrix elasticity directs stem cell lineage specification. Cell. 2006 Aug 25;126(4):677-89. PubMed PMID: 16923388. Epub 2006/08/23. eng.

5. Algahtani MS, Scurr DJ, Hook AL, Anderson DG, Langer RS, Burley JC, et al. High- throughput screening for biomaterials discovery. Journal of controlled release: official journal of the Controlled Release Society. 2014 Sep 28;190:115-26. PubMed PMID: 24993427. Epub 2014/07/06. eng.

6. Raza A, Lin CC. The influence of matrix degradation and functionality on cell survival and morphogenesis in PEG-based hydrogels. Macromolecular bioscience. 2013 Aug;13 (8):1048-58. PubMed PMID: 23776086. Pubmed Central PMCID: PMC3819142. Epub 2013/06/19. eng.

7. Khetan S, Guvendiren M, Legant WR, Cohen DM, Chen CS, Burdick JA. Degradation-mediated cellular traction directs stem cell fate in covalently crosslinked threedimensional hydrogels. Nature materials. 2013 May;12(5):458-65. PubMed PMID: 23524375. Pubmed Central PMCID: PMC3633615. Epub 2013/03/26. eng.

8. Swift J, Ivanovska IL, Buxboim A, Harada T, Dingal PC, Pinter J, et al. Nuclear lamin-A scales with tissue stiffness and enhances matrix-directed differentiation. Science (New York, NY). 2013 Aug 30; 341(6149): 1240104. PubMed PMID: 23990565. Pubmed Central PMCID: PMC3976548. Epub 2013/08/31. eng.

9. Feller L, Jadwat Y, Khammissa RA, Meyerov R, Schechter I, Lemmer J. Cellular Responses Evoked by Different Surface Characteristics of Intraosseous Titanium Implants. Biomed Res Int. 2015;2015:171945. PubMed PMID: 25767803. Pubmed Central PMCID: PMC4341860. Epub 2015/03/15. Eng.

10. Dalby MJ, Gadegard N, Oreffo RO. Harnessing nanotopography and integrin-matrix interactions to influence stem cell fate. Nature materials. 2014 Jun;13(6):558-69. PubMed PMID: 24845995. Epub 2014/05/23. eng.

11. Downing TL, Soto J, Morez C, Houssin T, Fritz A, Yuan F, et al. Biophysical regulation of epigenetic state and cell reprogramming. Nature materials. 2013 Dec;12(12): 1154-62. PubMed PMID: 24141451. Epub 2013/10/22. eng.

12. Mei Y, Saha K, Bogatyrev SR, Yang J, Hook AL, Kalcioglu ZI, et al. Combinatorial development of biomaterials for clonal growth of human pluripotent stem cells. Nature materials. 2010 Sep;9(9):768-78. PubMed PMID: 20729850. Pubmed Central PMCID: PMC3388774. Epub 2010/08/24. eng.

13. Khan F, Tare RS, Kanczler JM, Oreffo RO, Bradley M. Strategies for cell manipulation and skeletal tissue engineering using high-throughput polymer blend formulation and microarray techniques. Biomaterials. 2010 Mar;31(8):2216-28. PubMed PMID: 20056271. Epub 2010/01/09. eng.

14. Chou SY, KrausS PR, Renstrom PJ. Nanoimprint lithography. Journal of Vacuum Science gamp; Technology B. 1996;14(6):4129-33.

15. Kolind K, Dolatshahi-Pirouz A, Lovmand J, Pedersen FS, Foss M, Besenbacher F. A combinatorial screening of human fibroblast responses on microstructured surfaces. Biomaterials. 2010 Dec;31(35): 9182-91. PubMed PMID: 20832853. Epub 2010/09/14. eng.

16. Li W, Tang QY, Jadhav AD, Narang A, Qian WX, Shi P, et al. Large-scale topographical screen for investigation of physical neural-guidance cues. Scientific reports. 2015; 5:8644. PubMed PMID: 25728549. Pubmed Central PMCID: PMC4345323. Epub 2015/03/03. eng. 
17. Unadkat HV, Hulsman M, Cornelissen K, Papenburg BJ, Truckenmuller RK, Carpenter AE, et al. An algorithmbased topographical biomaterials library to instruct cell fate. Proceedings of the National Academy of Sciences of the United States of America. 2011 Oct 4;108(40):16565-70. PubMed PMID: 21949368. Pubmed Central PMCID: PMC3189082. Epub 2011/09/29. eng. 
CHAPTER $\mathbf{2}$

\section{HIGH-CONTENT IMAGING}

Frits Hulshof ${ }^{1}$, Er Liu 2,4, Andrea Negro5, Samy Gobaa ${ }^{5}$, Matthias Lutolf5, Prabhas V. Moghe 2,3 and Hugo Fernandes.

'Department Tissue Regeneration, MIRA Institute for Biomedical Technology and Technical Medicine, University of Twente, Enschede, The Netherlands.

${ }^{2}$ Department of Biomedical Engineering, ${ }^{3}$ Department of Chemical \& Biochemical Engineering,

${ }^{4}$ La Jolla Bioengineering Institute, San Diego, CA, USA 92121.

${ }^{5}$ Laboratory of Stem Cell Bioengineering and Institute of Bioengineering, Ecole Polytechnique Fédérale

de Lausanne, Lausanne, Switzerland. 


\section{Scope}

High-content imaging $(\mathrm{HCl}$ ) plays a pivotal role in high-throughput screening (HTS) of biological responses to biomaterials. Many of the imaging and image analysis techniques discussed in this chapter were used throughout this thesis. This chapter gives a brief introduction to the basic principles of high-content imaging by explaining the most commonly used imaging techniques, describing the general structure of an image analysis pipeline and providing a summary of available $\mathrm{HCl}$ software. In particular the initial steps of image analysis such as image correction, segmentation and feature extraction is explained in detail. Finally, an overview is provided of landmark examples of $\mathrm{HCl}$ and HTS in biomaterials science and future prospects.

\section{Origins of high-content imaging}

The first person to observe micro-organisms with a microscopic device was the Dutchman Anthon van Leeuwenhoek [1]. He was able to make and polish tiny lenses of high curvature which were the frontrunners of the modern microscope. Robert Hooke, the English father of microscopy, later on improved on the design and confirmed van Leeuwenhoek's findings. Since then, light microscopy has undergone several developments leading to modern light microscopy. The first use of fluorescence microscopy in biology was in 1881 when the bacteriologist Paul Ehrlich used fluorescin to observe the aqueous humour in the eye. The first immuno-staining was performed in 1950 by Melvin Kaplan [2]. Green Fluorescent protein (GFP) was the first fluorescent protein used in biology. GFP was isolated in 1961 by Shimomura and co-workers from jellyfish and it was cloned by Prisher in 1992. The ability to either use antibody conjugated fluorophores or to clone GFP into chimeric proteins led to the implementation of the fluorescence microscope in modern biology. High-content imaging ( $\mathrm{HCl}$ ) is the automation of fluorescence microscopy whereby the manual interpretation of images is replaced by computer algorithms.

The origins of $\mathrm{HCl}$ can be traced to the drug screening industry where it was developed more than fifteen years ago. The possibility to automatically acquire images from thousands of individual wells, which contain various compounds, became paramount to the pharmaceutical industry. Prior to automation, one of the major hurdles reside on the analyses and interpretation of images by human experts, therefore introducing unwanted variations in addition to the time required to analyze large data sets.

The drug screening industry realized that $\mathrm{HCl}$ has several advantages over other more commonly used high-throughput techniques such as fluorescence assisted cell sorting (FACS) and gene expression profiling with microarrays or next generation sequencing. The mentioned techniques often lack the ability to resolve the spatial and temporal features [3]. HCl not only provides the needed spatial and temporal features but also enables the tracking of organelles or even specific receptors. The academic world quickly caught on with the pharmaceutical industry in the development of $\mathrm{HCl}$ [4]. One of the great advantages of imaging is the ability to analyze single cells instead of averages of cell populations. This enhances the measurement of biological events, since a cell population is never homogenous and taking the average data of a population masks interesting phenotypes of individual cells. The data generated by $\mathrm{HCl}$ is more consistent, output can be standardized, and it can be stored in online databases, where it can be easily retrieved and interrogated [5]. A disadvantage of imaging is that is can be difficult to obtain strong statistical power in discriminating a dataset. To overcome this, it is vital to achieve clear and distinct readouts with strong controls. Therefore, significant attention has to be payed to the development of robust assays. Another disadvantage is the sheer size of the datasets resulting from large-scale, $\mathrm{HCl}$ experiments. Not 
only is the size of the raw data often daunting but the amount of data is also multiplied by every correction step. The average server computer is often unable to handle the data within a realistic timeframe, creating the need for a cluster computer setup and effective data logistics.

\section{High-content imaging techniques}

Most of the conventional $\mathrm{HCl}$ systems are based on fluorescence microscopy. The basic principle of these image based-assays is very simple: intensity quantification and relative locations of fluorophores in the specimen. This means that it is possible to visualize many different biological molecules, since fluorophores can be conjugated to many different substrates such as proteins, nucleotides and lipids. Alternatively, fluorescent molecules can be indirectly bound to targets by using labelled antibodies or other peptides with specific binding properties. Figure 1 shows examples of fluorescent markers used in HCl. Alternatively, reporter cell lines can be used which make $\mathrm{HCl}$ experiments more robust for two reasons. Firstly, there is no need for immuno-staining, which removes possible heterogeneity. Secondly, cell-line populations are generally more homogenous than other (primary) cell types.
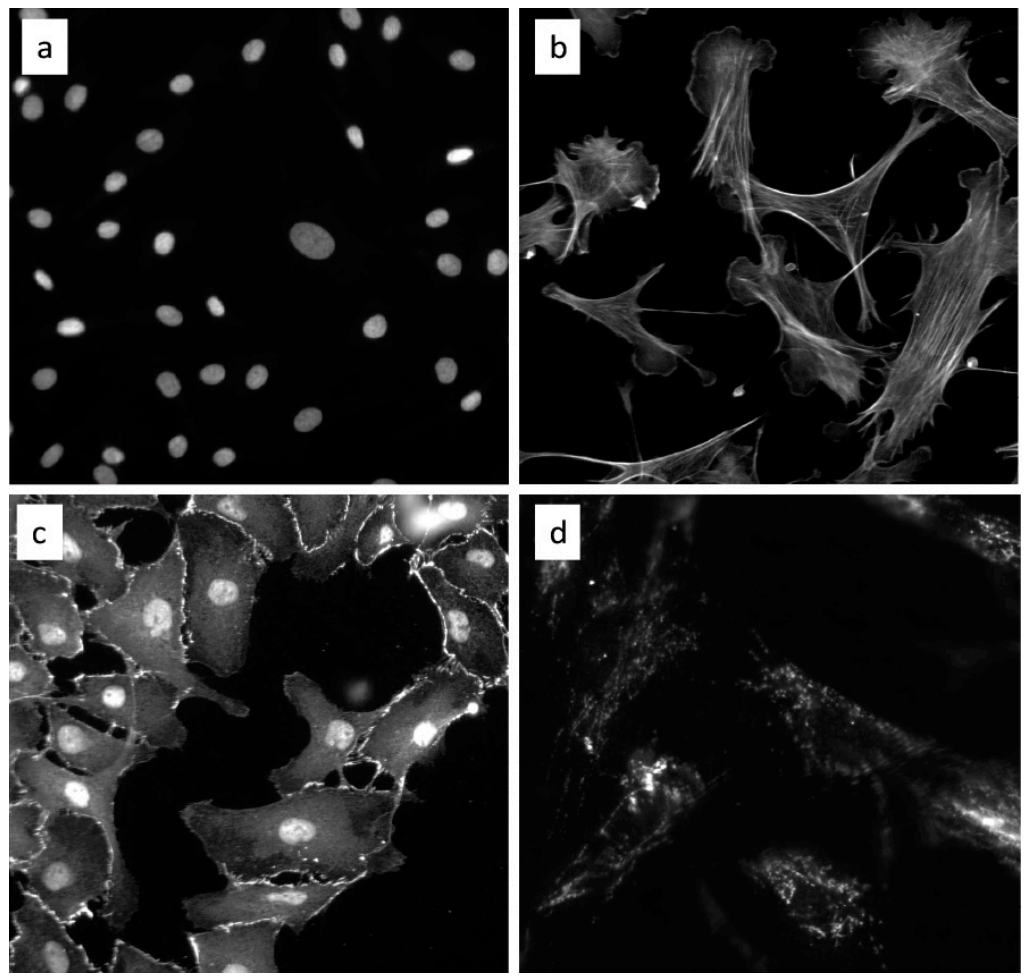

Figure 1: Images of various cells stained with commonly used markers. a: 4',6-diamidino-2-phenylindole (Dapi) marks the nuclei of cell by binding to DNA. It is commonly used to quantify cells or as nuclear counterstain. b: Phalloidin is a peptide toxin that specifically binds filamentous actin. Phalloidin, coupled to a fluorophore, is a very useful tool to visualize actin stress fibers and lamellipodia and it is also used to observe cell shape. c: Zona Occludens 1 (Z01) is a protein that is part of tight-junction complexes. Immuno-staining $Z 01$ is used to visualize tight junctions and the interconnectivity of cell growing in a monolayer. d: tissue non-specific alkaline phosphatase (ALP) is a protein that is often used as an early marker for osteogenic differentiation. 
Currently the most common instrument for fluorescence microscopy is an inverted fluorescence widefield microscope. For high-throughput screens such microscopes need to be fully automated. A fully motorized stage and a filter cube turret or filter wheels are essential. Alternatively, when an assay is designed on a multi-well plate platform, an automated microscope, specifically designed for $\mathrm{HCl}$ can be advantageous, since these are optimized for multi-well plates and can also facilitate live cell imaging when fitted with life cell imaging options. Line-scanning-microscopes, which were originally developed for the imaging tissue slides in pathology have been fitted with fluorescence imaging capabilities to make them interesting for life science. These systems can be interesting for HTS because they can image very large areas (multiple $\mathrm{cm}^{2}$ ) in short amounts of time to generate large datasets. The line-scanning microscopes usually have the drawbacks of a sacrifice in resolution, and very strict demands for sample dimensions.

Another instrument suitable for $\mathrm{HCl}$ is the confocal microscope. Confocal microscopy is able to increase the image quality, compared to wide-field systems. Since the confocal system only detects light of a single z plane, it is possible to make image stacks of cells. Such image stacks can be de-convolved by specific algorithms which are able to determine the points from which the scattered light originates. This enables the study of subcellular features in high detail. The resolution in 'conventional' fluorescence microscopy techniques is limited by the diffraction limit of light. In effect, this means that objects that are smaller than 100-200 nm cannot be distinguished. Super resolution fluorescence microscopy techniques are currently not suitable for High-throughput applications because of their long image acquisition times.

Most high-throughput experiments are done on fixed samples. Even though fixation introduces certain artefacts in the sample [6], it greatly simplifies the imaging process and makes it more robust. The option to use live cell imaging provides new possibilities as well as new challenges. For example, it enables the study of changes in protein localization or dynamic processes such as receptor internalization. For live cell imaging, the imaging conditions have to be optimal in order to not expose the cells or tissue to excessive light, which would cause photo bleaching and photo-toxicity.

Imaging of cells and tissues on biomaterials brings additional challenges. Often material-cell interfaces do not form the necessary flat surface that is essential for high-quality fluorescence imaging. Uneven surfaces cause parts of the object to be out of focus and can also cause problems for the autofocus systems in automated setups. Often there is a need to study the material-cell interface in a 3D environment. A 3D environment is prone to all of the above-discussed problems for uneven surfaces and it is therefore recommended the use a confocal system to capture the added z-plane typical of a 3D system. A priori design of 3D systems with materials surfaces and structures suitable for $\mathrm{HCl}$ is critical for good image acquisition. As mentioned earlier a flat surface is preferable, and it also greatly simplifies automation if single units in an array have a uniform size and are spaced in a rational and consistent manner.

\section{The image analysis pipeline}

When the 'raw' images have been acquired by the microscope, the image analysis takes place. Typically this data goes through a so-called image analysis 'pipeline' which starts with the raw data and ends with a dataset or even a first overview of the results. Between the beginning and the end of the pipeline, there are several steps. A typical pipeline contains elements which perform the tasks shown in figure 2 . These tasks or steps are described in this chapter except for the data analysis which is not covered in detail here, because it relies heavily on mathematics, bioinformatics and advanced statistics. The first step, in the pipeline is generally the image correction or image pre-processing step. In this step the dataset is corrected for systematic errors caused by the imaging equipment, errors caused by the limitations of the assay and bio- 
logical variance. Flat field correction is often required to compensate for uneven illumination of the sample caused by the spherical shape of the light bundle. This can be corrected by calculating the light curvature from a reference image and using the calculated curve to correct the dataset. Alternatively, algorithms which quantify the abnormal lighting from the dataset itself and correct the uneven illumination can be used. Other systemic errors such as well-to-well variation and plate-to-plate variation have to be corrected. Well-to-well variation is often corrected by the median polish method which corrects the data set stepwise by the median value in the data. In general, the higher the quality of the raw data, the less pre-processing required. The main function of the pre-processing stage is to increase the quality of the subsequent image segmentation and to make the segmentation more robust. Once the images have been corrected, they are ready for segmentation. Segmentation is a process of partitioning an image into multiple non-intersecting regions (often called regions of interest or Rols) such that each region is homogenous and the union of no two adjacent regions is homogenous [7].

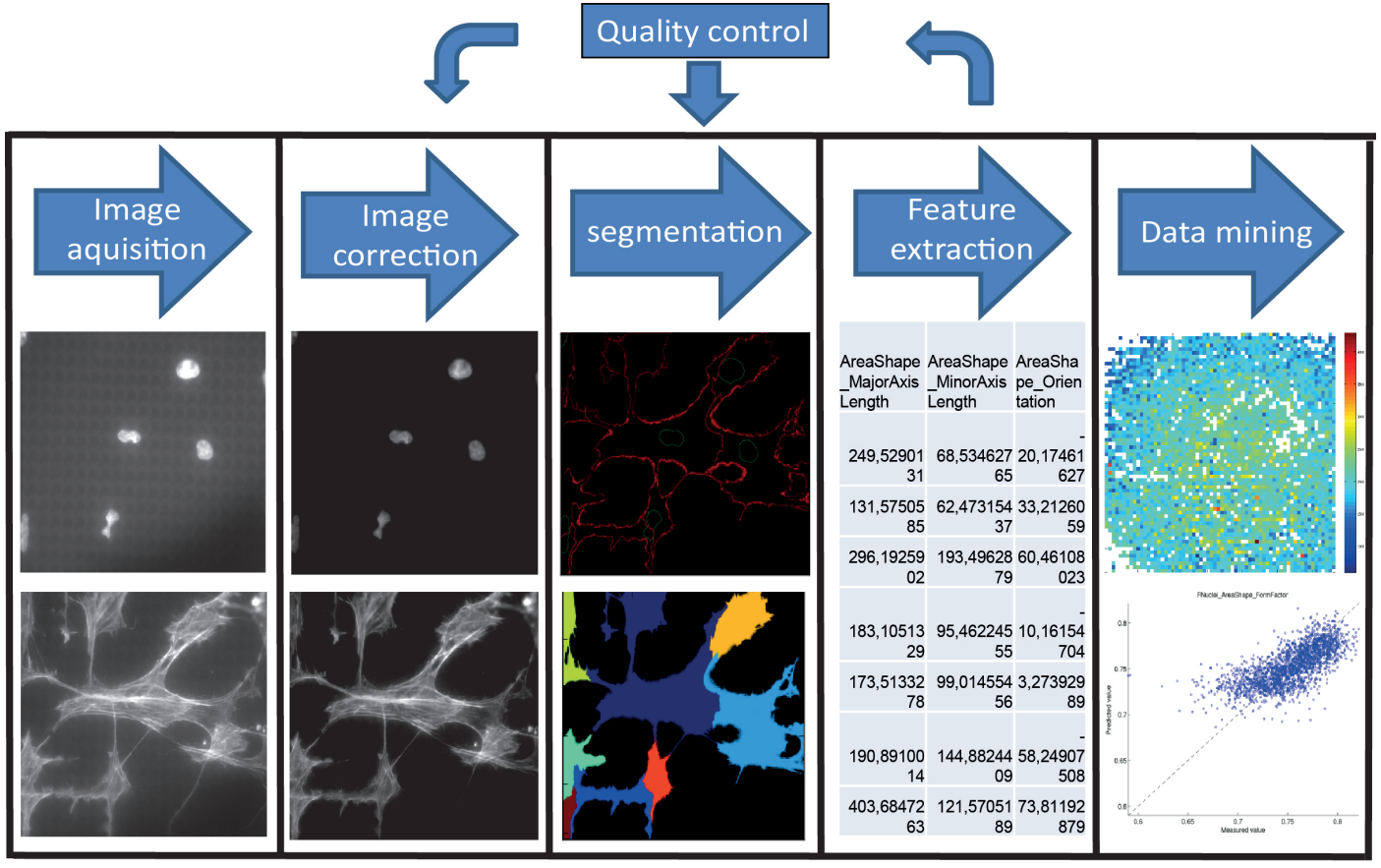

Figure 2: Schematic overview of an image analysis pipeline. Images of hMSCs with nuclear and filamentous actin stains.

Segmentation images created with cell profiler.

In this step, it is determined which aspects of the images will be quantified. Effectively the pixels within the image are divided into different populations on the basis of their intensity and (relative) positions. simple examples of quantifiable features include cell number or number of nuclei for a proliferation assay, for which a nuclear probe such as 4',6-diamidino-2-phenylindole (Dapi) is commonly used (Fig. 1a). Generally, in $\mathrm{HCl}$, more complex features such as details in cell morphology, cell orientation and marker localization in phenotypic screens are studied. These complex details in cell morphology are sometimes also termed morphometric descriptors. Examples of these are average cell area, perimeter, and relative length of major and minor axes [8]. Filamentous actin, directly labelled with a actin-GFP plasmid transfection or indirectly labelled with phalloidin (Fig. 1b), for example, can be used to measure these cell morphological parameters. The expression of certain markers can be measured, such as Alkaline phosphatase (Fig. d) which is associated with bone differentiation of hMSCs or Zona Occludens 1 (Fig 1c), which part of func- 
tional tight-junctions in epithelial cell-layers. Correct image segmentation is very important as errors in this step lead to faulty metrics in the feature extraction step.

During the feature extraction step, the regions that have been identified in the segmentation step are measured and collected in datasets or databases. In this step, many metrics such as relative shapes of cells or subcellular features, intensities, intensity distributions and texture can be measured. Examples from the literature include: the determination of cell numbers on polymer surfaces [9-11], details in cytoskeletal morphology [8, 12], sub-cellular features [5, 13], cytoskeletal features [14] and autophagy [15].

Between different steps in the pipeline, it is important to implement some form of quality control to assess the quality of the assay, the segmentation and the data set as a whole. The performance and the sensitivity of the assay has to be determined. There are many statistical methods that can be used [16]. One of the most common methods to predict assay performance is the $Z^{\prime}$ factor. The $Z^{\prime}$ factor is able to compare different assays and screens by using control wells ( $\left.Z^{\prime}\right)$ and sample wells ( $Z$ ).

To assess the sensitivity of an assay, most often the minimum significance ratio (MSR) is calculated. The MSR gives a measure for the reproducibility of potency values. When the quality of the assay is within the desired range it is ready for analysis. In the final step, the dataset can be analyzed to identify hits, look for correlations between parameters and look for other trends and relationships. The data mining of large datasets requires powerful analysis software tools and advanced statistics.

\section{Software}

In the years since $\mathrm{HCl}$ first made its way into academic research, many groups developing the technology also developed their own image analysis software. Several groups also published the capabilities of their software and made them freely available to the scientific community. Examples of free available packages developed by academic groups include HCDC-HITS [17], Micropilot [18] (also requires Labview commercial software) and EB/Image [19].

In 'medium' high-throughput approaches, research groups often use different software packages for different stages of the image analysis pipeline. For example, image acquisition is performed using software from the microscope manufacturer, while image correction and data analysis are performed using other software. In contrast, when research groups perform high-throughput experiments on a regular basis, it is more common to have the whole pipeline or workflow automated by the same software package to reduce manual work to a minimum.

Microscope manufacturers have also started providing comprehensive software packages for HCI with their microscope products. Often these software packages work well, but lack flexibility. Often the source-code is not open source, meaning that it is impossible to modify the software freely.

Examples of open source software programs that are often used for certain elements of the image analysis pipeline are Imagej and Cell Profiler. Many groups use shared or self-build plug-ins for Imagej to handle many pre-processing, segmentation and measurement steps [15]. Image) is a very flexible, light-weight, open source platform for image handling and analysis [20]. However, creating and editing scripts for Image J does require some simple knowledge of programming which the majority of biological scientists do not have. Fortunately, there is a large community of imaging experts who write and freely share their plug-ins for Imagej to add functionality and help those scientists with insufficient experience in programming.

Cell Profiler is a program that was developed as a flexible tool to make image processing and analysis more accessible to those scientists without comprehensive programming experience [21]. Cell profiler 
allows the user to design image analysis pipelines by combining many different modules which are able to apply correction, segmentation and feature extraction steps on large image sets. Every module is customizable to improve flexibility and new features are added in through regular updates.

\section{High-content imaging in materiomics}

In the last few years of $\mathrm{HCl}$ the emphasis has shifted from increasing screening capacity (quantity) to increasing content and combining technologies (quality) [22]. An example of combining technologies is the merge of high-throughput small RNA's assays and $\mathrm{HCl}$. Genome wide libraries of small interfering RNA's (siRNA) enable the selective knockdown of expressed proteins. Multi-parametric imaging has been used to associate specific protein deficiencies with cell phenotypes [12]. To increase the specificity of $\mathrm{HCl}$ assays, FRET sensors are increasingly being used. For example FRET sensors have been used to detect live cell caspase activation. Combining several caspase sensors enabled the observation of multiple decisive stages of cell death [23].

The trend of multiplexing $\mathrm{HCl}$ with other technologies is clearly present in the biomaterials field, enabling high-throughput biomaterials research or "materiomics". The field of materiomics comprises the study of structure-activity relations of an array of materials with physiochemical and biological properties. As biomaterials have evolved from design of a small number of disparate materials to larger libraries of combinatorial engineered materials, $\mathrm{HCl}$ approaches can now be more widely deployed to dissect cell-biomaterial interactions. Several groups in the biomaterials field have proposed various methods on the use of $\mathrm{HCl}$ of cells. A brief summary of selected methods appears below. The methods chosen vary from straightforward methods to determine cell numbers attached to substrates, for example, to more complex methods to determine cell morphology and expression of molecular markers.

For example, the Anderson group has published several articles on the applications of their polymer microarrays for HTS. Applications include the investigation of the clonal growth of embryonic stem cells [24], embryonic body adhesion [9] and Islet of Langerhans attachment [10].

Laser scanning cytometry (LSC) [25] was used to study cell adhesion and expression of markers of certain cell types on various polymers. In essence, LSC is similar to flow cytometry in that it identifies individual cells before measurements. The main difference is that LSC works on cells on flat surfaces instead of in a capillary flow. As a technique it is suitable for (semi) high-throughput quantitative imaging of flat surfaces, allowing rapid quantification of cell numbers and cellular fluorescence intensities. However, LSC lacks the resolution and optical sectioning of confocal microscopy necessary for ultra-structural analysis of subcellular features.

The Bradley group also used microarrays of spotted polymer blends to study cell-biomaterial interactions in High-throughput formats. Instead of LSC, an automated inverted fluorescence microscope is used to determine cell numbers on different polymer blends. Examples of the cellular parameters quantified in these studies include: the attachment of K562 human erythroleukimic suspension cells [26], L929 mouse fibrosarcoma cells [11] and human skeletal stem cells on polymer blend spots [27]. In contrast to these approaches, biomaterial-cell interactions have been extracted by discerning complex cellular parameters such as various intensity, texture, and geometric details of cellular and intracellular morphology and organization [8]. This work is an effective example of quantitative measurements of complex cellular parameters in the context of biomaterial research. In this study, GFP reporter cell lines were used in lieu of analysing fixed cells labelled with antibody-based immuno-staining and various GFP plasmids were used to stain different adhesion and cytoskeletal molecules in the cell such as actin, microtubules, plasma membrane 
and focal adhesions. Fixed cells were imaged by a motorized confocal microscope. Using image processing, the various cytoskeletal elements were segmented, measured and combined to create morphometric descriptors. These morphometric descriptors were able to describe many features of the cells, which could then be coupled to the substrate material properties. In subsequent work similar, morphometric descriptors - based on the actin cytoskeleton - were used to forecast stem cell lineage fates that were induced by various substrata and growth factors [14].

High-throughput $\mathrm{HCl}$ has been used to study the effects of microtopographies on multiple cellular parameters such as marker expression [28] and cell morphology. Previous work concerning the biological effects of nanoscale [29] and microscale topographies [30] also used fluorescence microscopy, but not in a high-throughput, high-content fashion. To enable HTS, cells were cultured on a polymeric surface, containing thousands of randomly generated topographies (Topochip) and imaged by fluorescence microscopy. The various topographies in 300x300 $\mu^{2}{ }^{2}$ "microwells" (TopoUnits) were arrayed 66 by 66 on a $2 \times 2 \mathrm{~cm}^{2}$ chip. Because of the constant dimensions in the Topochips design, the automated microscope (BD Pathway 435), which is optimized for well-plates, could be programmed to automatically image every TopoUnit in multiple channels. This could be achieved by creating a "coordinate map" of the TopoUnits and perfectly aligning the Topochip placed in the microscope to this map. The resulting images were analysed using a custom cell profiler pipeline. Analysis showed significant effects of the different microtopographies on cell morphology and ALP expression of hMSCS [28]. The Topochip HTS platform is the key tool used in this thesis to identify and study bioactive micro-topograhies.

Finally, HCl has been used to study cell - biomaterial interactions on soft hydrogel microwell arrays [31]. A motorized fluorescence microscope with life cell imaging capabilities was used. This made it possible to obtain phase contrast images of the arrays at early time points, before fixing the cells for immuno-staining at final time-points. Human mesenchymal stromal cells and mouse neural stem cells were stained for various markers to quantify differentiation into different lineages. The marker intensities were quantified and used as a measure of differentiation.

\section{Future prospects for high-content imaging techniques in materiomics}

The impact of material-based combinatorial libraries will be understood only with the advent of methods that can rapidly evaluate the impact of those thousands of combinations in cell fate. Currently, as the biological field entered the 'omics' era, many imaging techniques are being integrated in more high-throughput and/or high-content formats. Some of these techniques have recently been published and show promise for application in the biomaterials field. In this final part of the chapter, advances in two of these methods - FLIM FRET and Raman spectroscopy - will be briefly reviewed.

There are many ways to detect FRET; the two most common approaches are spectral radiometric imaging and fluorescence lifetime imaging (FLIM) [32]. Spectral radiometric imaging detects FRET by measuring intensities and is straightforward to implement instrumentally and has relatively short times. The major drawback is that the complete experimental setup has to be extensively calibrated, from the optical system itself to the actual sample. FLIM detects FRET by measuring the lifetime, rather than the intensity of the fluorescent signal, leading to a more robust measurement because it is usually independent of fluorophore concentration, excitation and detection efficiencies. However, FLIM has the drawbacks of relatively longer acquisition times and a requirement for more specialized instrumentation. In spite of these shortcomings, FLIM FRET has recently been applied in a high-throughput, high-content approach for drug discovery [32]. In this work, both high-throughput well-formats and multiplexed high-content setups were explored. A platereader was modified with a Nipkow disk for optical sectioning and used to study the dimerization of 
HIV-1 gag proteins in a High-throughput manner. This approach of modifying technology designed for HTS to quickly measure detailed sub cellular events is an example of the development of novel $\mathrm{HCl}$ technology. The application of FRET to study protein interactions or develop biosensors in high-throughput could provide complementary insights into intracellular biological signalling processes during active cellular remodelling on biomaterials. For example, a FRET calcium biosensor [33] could be used to measure the calcium content of progenitor cells on a biomaterial library as a metabolic or differentiation readout of developing cells (e.g. neurons or cardiomyocytes) in stimulatory micro-niches.

Raman spectrometry/spectroscopy, a widely used analytical tool in physics, chemistry and material science, has recently been explored for biomedical applications such as cancer diagnosis [34] and stem cell and regenerative medicine [35]. Raman scattering, originating from the inelastic scattering of a photon, is intrinsically weak and barely detectable by PMTs or CCD cameras that are generally used in optical imaging and microscopy. As a result, several techniques have been developed to enhance Raman signals to an extent that is suitable for imaging applications, including coherent anti-Stokes Raman spectroscopy (CARS) imaging that employs multiple photons to address the molecular vibration and produces a signal where the emitted waves are coherent with one another [36], surface-enhanced Raman spectroscopy (SERS) imaging which utilizes nanoparticles (sometimes called SERS tags or SERS dots) as signal enhancement agents [37], and single-walled carbon nanotubes (SWNT) imaging which utilizes its intense intrinsic Raman scatter at $1593 \mathrm{~cm}^{-1}$ [38]. In the case of high-content/HTS where multiple biomarkers are usually needed to probe cell systems, SERS based imaging is especially relevant due to its enhanced multiplexing capabilities. In general, nanoparticle-based SERS tags as labels for antibodies or other ligands offer several potential advantages over traditional fluorophores [39]. First, like nanoparticle quantum dots, SERS tags are more photo-stable than conventional fluorescence dyes. Second, the nanoparticle resonance, and thus excitation wavelength, can be tuned by changing the size and shape of the nanoparticle, allowing the fabrication of NIR-excitable SERS tags that can reserve the space for fluorescent imaging at visible range. Finally, by using various Raman compounds that exhibit distinct Raman spectra, SERS tags can be prepared that have unique spectral signatures, thereby allowing a high level of multiplexing within a relatively narrow spectral range. As of now, SERS-based Raman imaging, although mostly only demonstrated in proof-of-concept experiments, could provide additional chemical/compositional information to aide future high-content screening research. Information can also be contextualized around the cell phenotypes on different biomaterials, for example, by attaching various molecular recognition agents to Raman tags to enable simultaneous probing of multiple biological events (cell survival, proliferation, apoptosis, differentiation, etc) 


\section{Literature}

1 Masters, B. R. (2008). History of the Optical Microscope in Cell Biology and Medicine. Encyclopedia of life sciences.

2 Masters, B. R. (2010). The Development of Fluorescence Microscopy. Encyclopedia of life sciences.

3 Kobel, S. \& Lutolf, M. (2010). High-throughput methods to define complex stem cell niches. Biotechniques, 48 , ix-xxii.

4 Bickle, M. (2010). The beautiful cell: high-content screening in drug discovery. Anal Bioanal Chem, 398, 219-26.

5 Conrad, C., Erfle, H., Warnat, P., Daigle, N., Lorch, T., Ellenberg, J., Pepperkok, R. \& Eils, R. (2004). Automatic identification of subcellular phenotypes on human cell arrays. Genome Res, 14, 1130-6.

6 Bhadriraju, K., Elliott, J. T., Nguyen, M. \& Plant, A. L. (2007). Quantifying myosin light chain phosphorylation in single adherent cells with automated fluorescence microscopy. BMC Cell Biol, 8, 43.

7 Pal, N. R. \& Pal, S. K. (1993). A Review on Image Segmentation Techniques. Pattern Recognition, 26,1277-1294.

8 Treiser, M. D., Liu, E., Dubin, R. A., Sung, H. J., Kohn, J. \& Moghe, P. V. (2007). Profiling cell-biomaterial interactions via cell-based fluororeporter imaging. Biotechniques, 43, 361-6, 368.

9 Yang, J., Mei, Y., Hook, A. L., Taylor, M., Urquhart, A. J., Bogatyrev, S. R., Langer, R., Anderson, D. G., Davies, M. C. \& Alexander, M. R. (2010). Polymer surface functionalities that control human embryoid body cell adhesion revealed by high-throughput surface characterization of combinatorial material microarrays. Biomaterials, 31, 8827-38.

10 Mei, Y., Hollister-Lock, J., Bogatyrev, S. R., Cho, S. W., Weir, G. C., Langer, R. \& Anderson, D. G. (2010). A high-throughput micro-array system of polymer surfaces for the manipulation of primary pancreatic islet cells. Biomaterials, 31, 8989-95.

11 Pernagallo, S., Diaz-Mochon, J. J. \& Bradley, M. (2009). A cooperative polymer-DNA microarray approach to biomaterial investigation. Lab Chip, 9, 397-403.

12 Fuchs, F., Pau, G., Kranz, D., Sklyar, O., Budjan, C., Steinbrink, S., Horn, T., Pedal, A., Huber, W. \& Boutros, M. (2010). Clustering phenotype populations by genome-wide RNAi and multiparametric imaging. Mol Syst Biol, 6, 370.

13 Bakal, C., Aach, J., Church, G. \& Perrimon, N. (2007). Quantitative morphological signatures define local signaling networks regulating cell morphology. Science, 316, 1753-6.

14 Treiser, M. D., Yang, E. H., Gordonov, S., Cohen, D. M., Androulakis, I. P., Kohn, J., Chen, C. S. \& Moghe, P. V. (2010). Cytoskeleton-based forecasting of stem cell lineage fates. Proc Natl Acad Sci U S A, 107, 610-5.

15 Kriston-Vizi, J., Lim, C. A., Condron, P., Chua, K., Wasser, M. \& Flotow, H. (2010). An automated high-content screening image analysis pipeline for the identification of selective autophagic inducers in human cancer cell lines. J Biomol Screen, 15, 869-81.

16 Inglese, J., Johnson, R. L., Simeonov, A., Xia, M., Zheng, W., Austin, C. P. \& Auld, D. S. (2007). High-throughput screening assays for the identification of chemical probes. Nat Chem Biol, 3, 466-79.

17 Kozak, K., Bakos, G., Hoff, A., Bennett, E., Dunican, D., Davies, A., Kelleher, D., Long, A. \& Csucs, G. (2010). Workflow-based software environment for large-scale biological experiments. J Biomol Screen, 15, 892-9.

18 Conrad, C., Wunsche, A., Tan, T. H., Bulkescher, J., Sieckmann, F., Verissimo, F., Edelstein, A., Walter, T., Liebel, U., Pepperkok, R. \& Ellenberg, J. (2011). Micropilot: automation of fluorescence microscopy-based imaging for systems biology. Nature Methods, 8, 246-U89.

19 Pau, G., Fuchs, F., Sklyar, O., Boutros, M. \& Huber, W. (2010). EBlmage--an R package for image processing with applications to cellular phenotypes. Bioinformatics, 26, 979-81.

20 Abramoff, M. D., Magelhaes, P. J. \& Ram, S. J. (2004). Image Processing with Imagej. Biophotonics International, 11, 36-42.

21 Carpenter, A. E., Jones, T. R., Lamprecht, M. R., Clarke, C., Kang, I. H., Friman, O., Guertin, D. A., Chang, J. H., Lindquist, R. A., Moffat, J., Golland, P. \& Sabatini, D. M. (2006). CellProfiler: image analysis software for identifying and quantifying cell phenotypes. Genome Biol, 7, R100.

22 Mayr, L. M. \& Bojanic, D. (2009). Novel trends in high-throughput screening. Curr Opin Pharmacol, 9, 580-8. 
23 Joseph, J., Seervi, M., Sobhan, P. K. \& Retnabai, S. T. (2011). High-throughput ratio imaging to profile caspase activity: potential application in multiparameter high content apoptosis analysis and drug screen-ing. PLoS One, 6, e20114.

24 Mei, Y., Saha, K., Bogatyrev, S. R., Yang, J., Hook, A. L., Kalcioglu, Z. I., Cho, S. W., Mitalipova, M., Pyzocha, N., Rojas, F., Van Vliet, K. J., Davies, M. C., Alexander, M. R., Langer, R., Jaenisch, R. \& Anderson, D. G. (2010). Combinatorial development of biomaterials for clonal growth of human pluripotent stem cells. Nat Mater, 9, 768-78.

25 Pozarowski, P., Holden, E. \& Darzynkiewicz, Z. (2006). Laser scanning cytometry: principles and applications. Methods Mol Biol, 319, 165-92.

26 Pernagallo, S., Unciti-Broceta, A., Diaz-Mochon, J. J. \& Bradley, M. (2008). Deciphering cellular morphology and biocompatibility using polymer microarrays. Biomed Mater, 3, 034112.

27 Khan, F., Tare, R. S., Kanczler, J. M., Oreffo, R. 0. \& Bradley, M. (2010). Strategies for cell manipulation and skeletal tissue engineering using high-throughput polymer blend formulation and microarray techniques. Biomaterials, 31, 2216-28.

28 Unadkat, H. V., Hulsman, M., Cornelissen, K., Papenburg, B. J., Truckenmuller, R. K., Carpenter, A. E., Wessling, M., Post, G. F., Uetz, M., Reinders, M. J., Stamatialis, D., Van Blitterswijk, C. A. \& De Boer, J. (2011). An algorithm-based topographical biomaterials library to instruct cell fate. Proc Natl Acad Sci U S A, 108, 16565-70.

29 Dalby, M. J., Gadegaard, N., Tare, R., Andar, A., Riehle, M. O., Herzyk, P., Wilkinson, C. D. \& Oreffo, R. O. (2007). The control of human mesenchymal cell differentiation using nanoscale symmetry and disorder. Nat Mater, 6, 997-1003.

30 Lovmand, J., Justesen, J., Foss, M., Lauridsen, R. H., Lovmand, M., Modin, C., Besenbacher, F., Pedersen, F. S. \& Duch, M. (2009). The use of combinatorial topographical libraries for the screening of enhanced osteogenic expression and mineralization. Biomaterials, 30, 2015-22.

31 Gobaa, S., Hoehnel, S., Roccio, M., Negro, A., Kobel, S. \& Lutolf, M. P. (2011). Artificial niche microarrays for probing single stem cell fate in high throughput. Nat Methods, 8, 949-55.

32 Kumar, S., Alibhai, D., Margineanu, A., Laine, R., Kennedy, G., Mcginty, J., Warren, S., Kelly, D., Alexandrov, Y., Munro, I., Talbot, C., Stuckey, D. W., Kimberly, C., Viellerobe, B., Lacombe, F., Lam, E. W., Taylor, H., Dallman, M. J., Stamp, G., Murray, E. J., Stuhmeier, F., Sardini, A., Katan, M., Elson, D. S., Neil, M. A., Dunsby, C. \& French, P. M. (2011). FLIM FRET technology for drug discovery: automated multiwell-plate highcontent analysis, multiplexed readouts and application in situ. Chemphyschem, 12, 609-26.

33 Mank, M., Reiff, D. F., Heim, N., Friedrich, M. W., Borst, A. \& Griesbeck, 0. (2006). A FRET-Based Calcium Biosensor with Fast Signal Kinetics and High Fluorescence Change. Biophysical Journal, 90, 1790-1796.

34 Zhang, Y., Hong, H. \& Cai, W. (2010). Imaging with Raman spectroscopy. Curr Pharm Biotechnol, 11, 654-61.

35 Schulze, H. G., Konorov, S. 0., Caron, N. J., Piret, J. M., Blades, M. W. \& Turner, R. F. (2010). Assessing differentiation status of human embryonic stem cells noninvasively using Raman microspectroscopy. Anal chem, 82, 5020-7.

36 Evans, C. L. \& Xie, X. S. (2008). Coherent anti-stokes Raman scattering microscopy: chemical imaging for biology and medicine. Annu Rev Anal Chem (Palo Alto Calif), 1, 883-909.

37 Schlucker, S. (2009). SERS microscopy: nanoparticle probes and biomedical applications. Chemphyschem, 10, $1344-54$.

38 Hong, H., Gao, T. \& Cai, W. (2009). Molecular Imaging with Single-Walled Carbon Nanotubes. Nano Today, 4, 252-261.

39 Nolan, J. P. \& Sebba, D. S. (2011). Surface-enhanced Raman scattering (SERS) cytometry. Methods Cell Biol, $102,515-32$. 


\section{MINING FOR OSTEOGENIC SURFACE TOPOGRAPHIES: \\ IN SILICO DESIGN TO IN VIVO OSSEO-INTEGRATION}

Frits Hulshof 1,2, Bernke Papenburg ${ }^{3}$, Aliaksei Vasilevic², Marc Hulsman ${ }^{4}$, Yiping Zhao ${ }^{3}$, Marloes Levers ${ }^{3}$, Natalie Fekete ${ }^{3}$, Meint de Boer ${ }^{6}$, Huipin Yuan ${ }^{7}$, Shantanu Singh ${ }^{5}$, Mark-Anthony Bray ${ }^{5}$, David J. Logan ${ }^{5}$, Marcel Reinders ${ }^{4}$, Anne E. Carpenter ${ }^{5}$, Clemens van Blitterswijk8 ${ }^{8}$, Dimitrios Stamatialis', Jan de Boer ${ }^{2}$

1MIRA Institute for Biomedical Technology and Technical Medicine, Department of Biomaterials Science and Technology, University of Twente, Enschede, The Netherlands;

${ }^{2}$ MERLN Institute for Technology-Inspired Regenerative Medicine, Department of Cell Biology -inspired Tissue Engineering, Maastricht, The Netherlands;

${ }^{3}$ Materiomics BV, Maastricht, The Netherlands;

${ }^{4}$ Delft Bioinformatics Lab, Delft University of Technology, Delft, The Netherlands;

IImaging Platform, Broad institute of MIT and Harvard, Cambridge, MA, USA;

${ }^{6} \mathrm{MESA}$ +Institute for Nanotechnology, University of Twente, Enschede, The Netherlands;

${ }^{7}$ Xpand biotechnology BV, Bilthoven, The Netherlands;

${ }^{8}$ MERLN Institute for Technology-Inspired Regenerative Medicine, Department of Complex Tissue Regeneration, University of Maastricht, Maastricht, The Netherlands. 


\section{Abstract}

Surface topography modulates cellular structure and functions such as proliferation, orientation, morphology, gene expression and differentiation. Modifying biomaterials surfaces with topography is a viable strategy to improve biomedical implant performance. We have identified osteogenic topographies to improve titanium (Ti) bone implants. Human mesenchymal stromal cells (hMSCs) were cultured on Ti-coated 'Topochips' which contain thousands of unique topography designs. Using highthroughput, high-content imaging we determined the effect of each topography on alkaline phosphatase (ALP) expression, cytoskeletal organization, and nuclear morphology. Topographies inducing high levels of ALP expression were fabricated on larger substrates and proofed to upregulate a selection of osteogenic markers and enhance mineralization. In a rabbit model, these osteogenic topographies showed improved bone bonding comparable to, and in one case, slightly better than the clinical benchmark: Straumann sand blasted, acid etched (SLA) topography. Moreover, we find that ALP expression correlates to both the design parameters of the topographies as well as the shape of the cells. Together, this work demonstrates the power of high-throughput screening to design and identify surface topographies.

\section{Introduction}

The use of biomaterials in biomedical applications has greatly improved the quality of life for many patients. The development of stents, pacemakers, orthopedic implants and catheters has been critical for successful clinical treatments. Nevertheless, each of these treatments is occasionally met with general complications such as biomaterial associated infections (1) or more specific problems such as micro-motion in the case of bone implants (2) or thrombosis in the case of stents (3). These and other biomaterials and devices need ongoing improvements but developing and identifying superior biomaterials for a specific application is a non-trivial challenge. There are many biomaterial properties that affect biological systems, such as surface charge, wettability, degradability, bulk stiffness, surface stiffness, surface chemistry, topography, etc..

Surface topography (in both micro- and nanometer scale) has been shown to evoke many in vitro cellular responses such as differentiation and proliferation of hMSCs $(4,5)$, differentiation of neural progenitor cells (6), and morphological changes in pre-osteoblastic cells (7). Methodological screening has identified topographies that affect fibroblast proliferation (8), pre-osteoblast mineralization (9) and neural guidance (10). As a tailorable biomaterial property for medical devices, surface topography has two major advantages. Firstly, it is relatively easy to translate to a clinical application because the necessary fabrication technologies already exist and it is relatively easy to conform to strict medical device regulation, unlike for chemical modifications or the addition of biologicals such as recombinant proteins (11). Secondly, surface topography has already proven to improve the performance of implants such as orthopedic implants and dental implants in the clinic. Recent works clearly showed that topography can improve osseo-integration $(12,13)$. In fact, it has become common practice that, for example hip implants are sand blasted and/or acid etched in order to obtain suitable topography which improves osseo-integration (14). However, these techniques lack control and often introduce steps that change the chemistry of the material (like acid etching).

Surface Topography is interconnected with other biomaterial properties, such as surface chemistry and can be difficult to tailor as an individual property. The knowledge and understanding of the underlying biological mechanisms of tissue-biomaterial interactions is generally empirical, and relatively limited. Because of this inherent complexity and lack of predictive theoretical framework, high-throughput combinatorial screening (HTCS) of biomaterial properties and of material-cell interactions are very appealing technolo- 
gies. HTCS has already uncovered culture substrates for maintenance of stem cell pluripotency $(15,16)$ and differentiation of human multipotent stromal cells (hMSCs) (17-19). Despite these advantages no systematic studies including high numbers of well-designed topographies have been conducted to determine the biological effect of surface topography.

Current micro-fabrication technologies allow specific control of surface topography to fabricate libraries of topographies for HTCS such as the 'TopoChip' high-throughput screening platform (19). Here, we apply the Topochip to design, produce and screen thousands of randomly generated topographies for improved osseo-integration. In order to design shapes in an unbiased way, an in silico library of more than 150 million topographies was generated by combining three primitive shapes: triangle, rectangle and circle. By combining the primitives in varying numbers and sizes, we can create any conceivable shape (Fig. 1a). From this library, 2176 topographical features were randomly selected and physically constructed in duplicate in $290 \times 290 \mu^{2} \mathrm{~m}^{2}$ 'TopoUnits' on a $2 \times 2 \mathrm{~cm}^{2}$ titanium-coated 'TopoChip' (Fig.1b,c) to match the application in titanium bone implants.

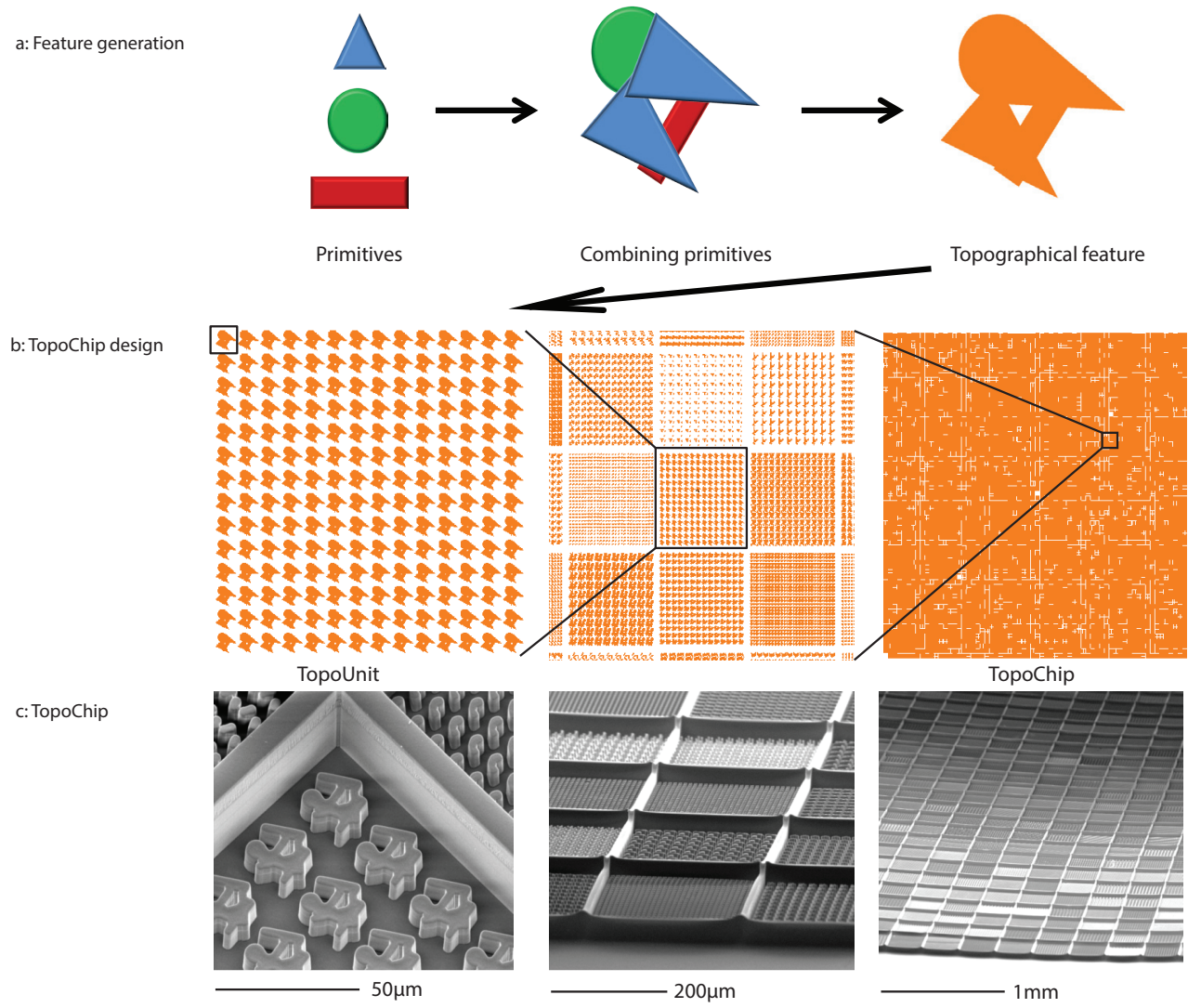

Figure 1: Topochip design. a: From left to right, the three primitive shapes, circle, triangle and rectangle are combined in varying numbers and dimensions, to form a random shape of a topographical feature. Range of parameters within the algorithm allows a theoretical library of topographies with 150 million different possible shapes. b: For the Topochip design 2176 topographies are randomly selected from the theoretical library and are arrayed in 66 by 66 in $290 \times 290 \mu \mathrm{m}^{2}$ TopoUnits. From left to right the illustrations zoom out from a single TopoUnit to a complete $2 \times 2 \mathrm{~cm}^{2}$ Topochip. c: Below the illustrations are SEM images with similar scaling. 
Screening hMSCs as a cell model allowed identification of instructive osteogenic topographies. In addition, by monitoring the effects of design parameters, we could infer relationships between topographical feature shapes, cell morphology and cell differentiation, analogous to identification of drug identification by computational chemistry (20). HMSCs were used as a cell model to identify osteogenic topographies as they are often used to study cell-biomaterial interactions $(21,22)$. Furthermore, it is already established that hMSCs will differentiate into osteoblasts and deposit bone tissue when implanted on a ceramic scaffold in vivo $(23,24)$. For screening and identification of the osteogenic designed topographies, we use a set of robust imaging and analytical tools. The osteogenic potential of candidate topographies was thoroughly evaluated in vitro and in vivo using a rabbit model.

\section{Results}

\section{Characterization of hMSCs on titanium-coated TopoChips}

To prepare for combinatorial screening, we first characterized the response of hMSCs (with verified multilineage potential, see Supplementary Fig. S1) to titanium-coated TopoChips, as we aimed to improve titanium bone implants. Fabrication of the Topochips included hot embossing of poly lactic acid (PLA) films and fabrication quality and reproducibility was confirmed by scanning electron microscopy (SEM) (Fig. 1c). We added a $200 \mathrm{~nm}$ layer of titanium, thin enough to preserve the topographical features (3 $\mathrm{\mu m}$ minimum dimensions) and cultured hMSCs on the Topochips for 5 days. We observed a wide variety of both nuclear and actin morphologies in response to the microtopographies (Fig. 2). Within a TopoUnit (indicated by yellow box in Figure 2a), the cells respond qualitatively in a very similar way.

\section{Screening identifies topographies that induce ALP expression of hMSCS}

After determining that the hMSCs respond well to the titanium-coated Topochips, a full Topochip screen was performed. Tissue non-specific alkaline phosphatase (ALP) was chosen as a screening marker for osteogenic differentiation. ALP is crucial for mineralization of bone because it generates the inorganic phosphate needed for hydroxyapatite crystallization (25). HMSCs were seeded on 8 titanium-coated TopoChips and cultured for 5 days after which the nuclei, filamentous actin cytoskeleton and ALP where fluorescently labeled. Before identification of potential hits, several quality control parameters were verified such as uniform cell distribution and inter-chip reproducibility. The amount of cells per unit follows a normal distribution associated with the stochastic cell seeding process (Fig. 3a). Cell morphology is highly reproducible between multiple Topochips used in the screen (Supplementary Fig. S2). To identify osteogenic topographies, ALP intensity was measured (see materials and methods) which resulted in a measurement distribution shown in Fig. 3b. The topographies inducing ALP intensity values at the ends of the $S$ curve were selected as positive and negative hit topographies: 15 topographies were selected consisting of 5 low intensity topographies and 10 high intensity topographies (Fig. 3c). The non-patterned (flat) surface was among the group of topographies with the lowest ALP intensity levels and was included as control. Positive hits had 2 to 3 times higher ALP intensity than the negative hits. In conclusion, the screening experiment led to the identification of a set of topographies which are able to modulate ALP expression. 
a

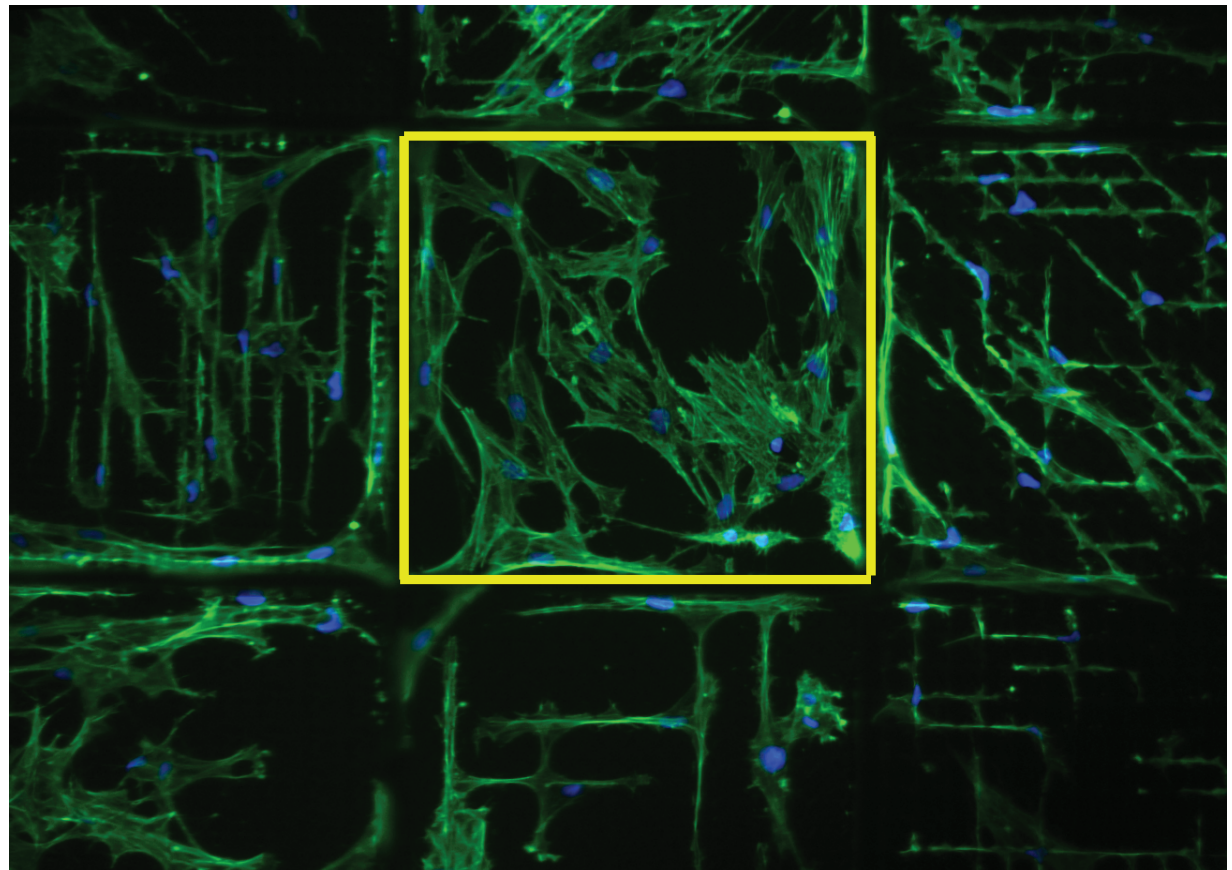

$100 \mu \mathrm{m}$

b
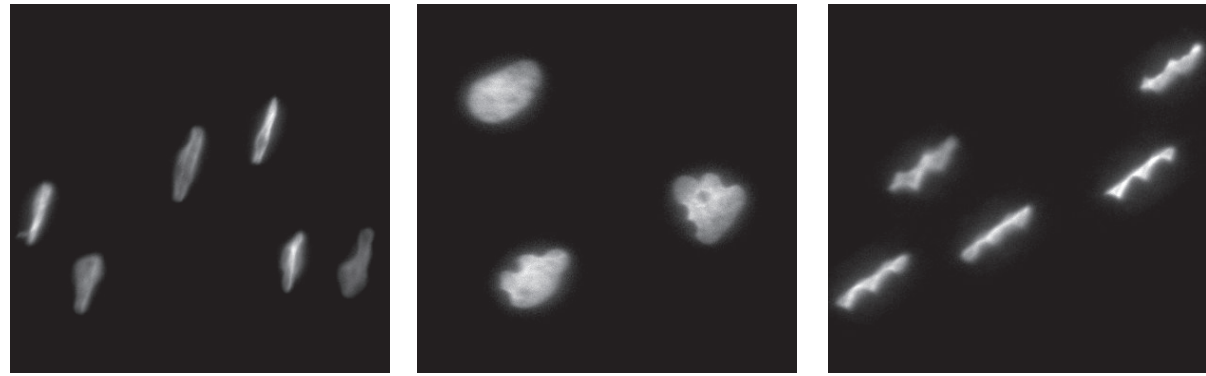

Figure 2: Effects of topography on hMSC. a: Fluorescent image from a random area on Topochip seeded with hMSCS.

The filamentous actin is shown in green and the nuclei in blue. The yellow square indicates a TopoUnit. b: Fluorescent images of nuclear morphologies which were severely affected by the topographies.

Figure 3:Identification of osteogenic topographies by HTS a: Cell distribution per chip in the screen. b: Distribution of ALP intensity corrected to actintegrated intensity. c: Average integrated Alp intensities of TopoUnits of selected hit topographies. d: Typical topographical shapes of hit topographies. e: Typical hMSC morphologies on hit topographies. 


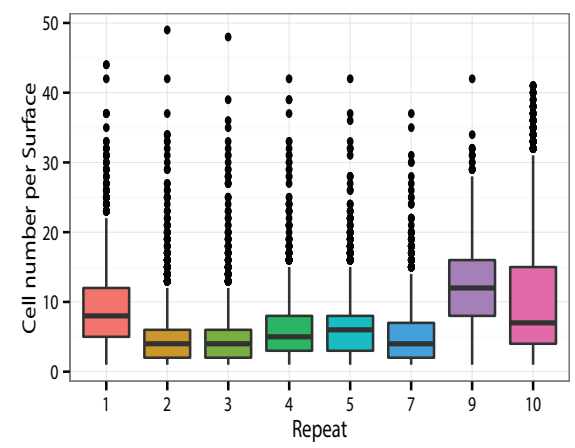

b

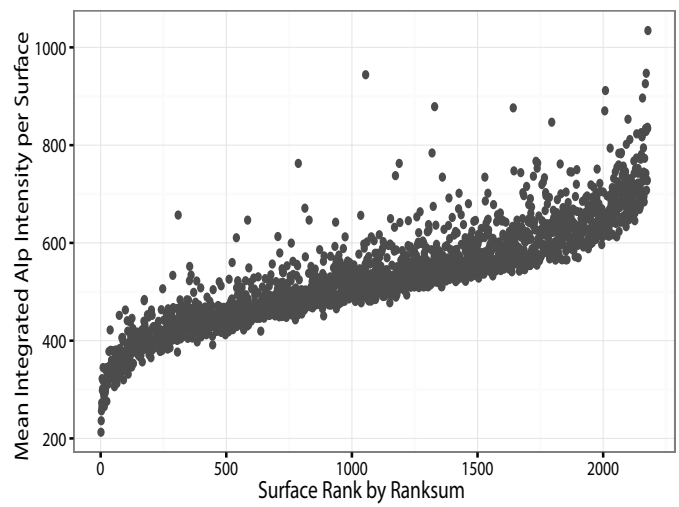

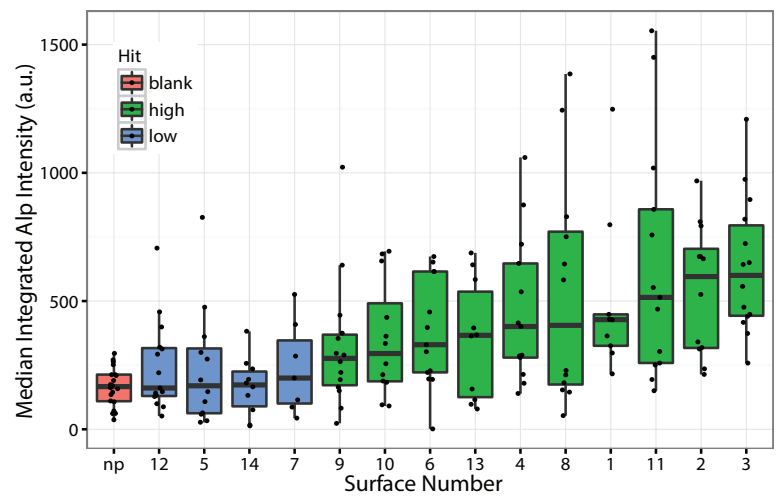

moderate spacing: positive

d

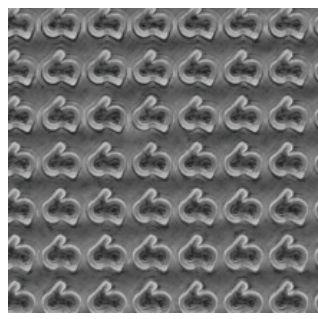

little spacing: negative

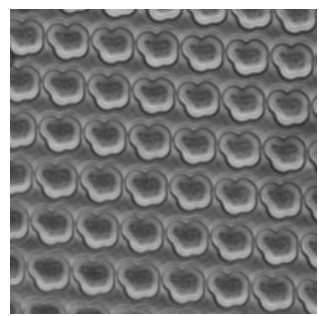

large spacing:

negative

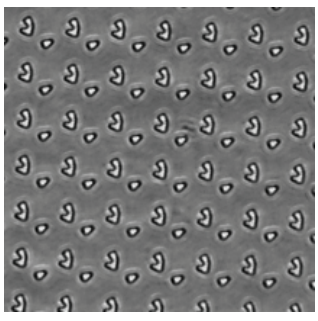

$100 \mu \mathrm{m}$
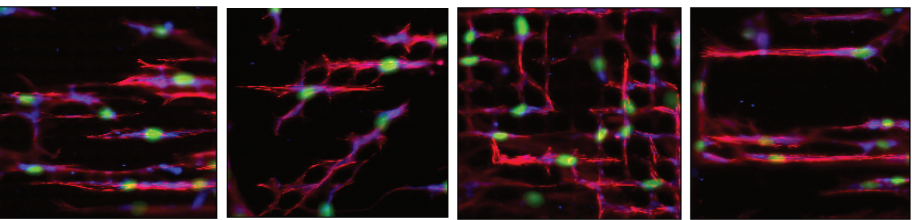

negative hits: Spread cells
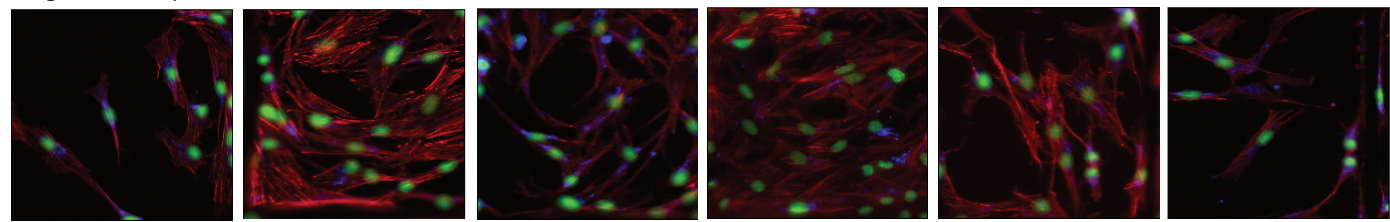

$100 \mu \mathrm{m}$ 


\section{ALP expression correlates with topographical shape parameters and cell morphology}

Visual inspection of the topographies that affect ALP expression hinted at certain trends. Low scoring units have two classes of topographies: relatively small features of several micrometers with very large spacing between the features (more than $15 \mu \mathrm{m}$ ) and average to large features of 10 to $30 \mu \mathrm{m}$ with very little spacing of only several micrometers (Fig. 3d). Both types of topographies instruct similar cell morphologies: generally flat spread cells, similar to a non-patterned surface. The high scoring features are generally average to large features, 10 to $30 \mu \mathrm{m}$, with moderate spacing of 5 to $10 \mu \mathrm{m}$. The cells are confined between the structures, are relatively dense and narrow compared to cells on a flat surface (Fig. 3e). These initial observations prompted us to utilize the Topochip screen data to identify relationships between ALP expression and cell morphology. To investigate this, one hundred surfaces with either the highest or the lowest median per surface ALP integrated intensity (the sum of all pixel intensities) were used. From the classification tree plot (Fig. 4a) we found a combination of cell shape parameters, measured by Cell Profiler (26), such as cell solidity, Euler Number and compactness as being able to separate the two types of surfaces. The Random Forest Classification algorithm, as described in the Methods section, was employed to create a robust model which can predict ALP expression based on cell shapes. The ROC curve of the predicted model (Fig. 4b) showed a tested accuracy of 70\%. In line with earlier visual observation, solidity and compactness were the most important cell shape features that were able to predict ALP expression (Fig. 4c) and they could be used to clearly separate the two surface classes (Fig. 4d). This figure clearly shows that cell solidity is best correlated with the separation between positive and negative hits which is in agreement with the morphological description of the positive cells in Figure 3e. Low cell solidity (calculated as 'the proportion of pixels in the convex hull that are also in the object') is caused by the fact that the cells are located around the features which to the software appear as large holes within the cell bodies.

To further illustrate the clear morphological difference between positive and negative hits, cell morphology of two of the positive hits, \#4 and \#8, and one negative hit, \#7, was analyzed in more detail by confocal microscopy (Fig. 4e) and was assessed by staining the nuclei and filamentous actin. In agreement with the screening experiment, the cells on the positive hit topographies are confined between the structures, leading to cells resembling a network of tubes. Cross sections of confocal image stacks (Fig. 4f) clearly show that the tube-like cells on the topographies are on average double the height (9.4 and $12 \mu \mathrm{m})$ of the cells on a flat surface $(4.8 \mu \mathrm{m})$ and on the negative hit surface $(6 \mu \mathrm{m})$. It could be speculated that the topographies affect hMSC differentiation by providing a vertical site of adherence in the form of sides/ walls of the topographies confining the cells and preventing flattening.

Our results show that cell morphology and ALP expression are correlated. Previously published Topochip data has shown that certain cell morphological parameters such as nuclear shape and cell area can be directly correlated to the design parameters of the topographies (20). Together these two findings, (1) feature design predicts cell morphology and (2) cell morphology predicts ALP expression, suggest that feature design parameters might be able to predict ALP expression directly. To investigate this further we selected 100 surfaces with the highest ALP expression and 100 surfaces with lowest ALP expression. A classification tree algorithm was used to characterize the data set (Fig. 5a). 
a

Positive

2 (EulerNumber

$-25.625 \geq-25.625$
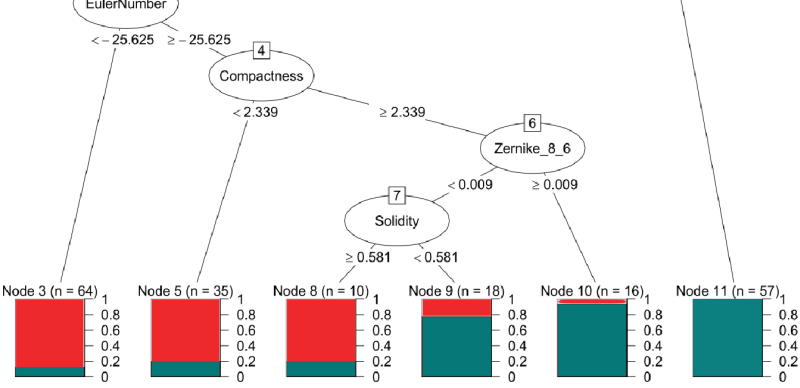

b

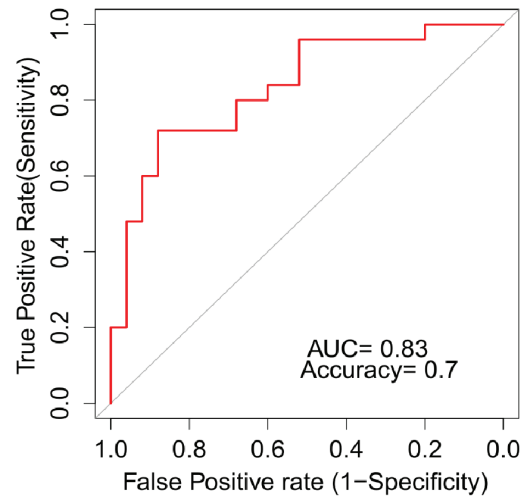

C

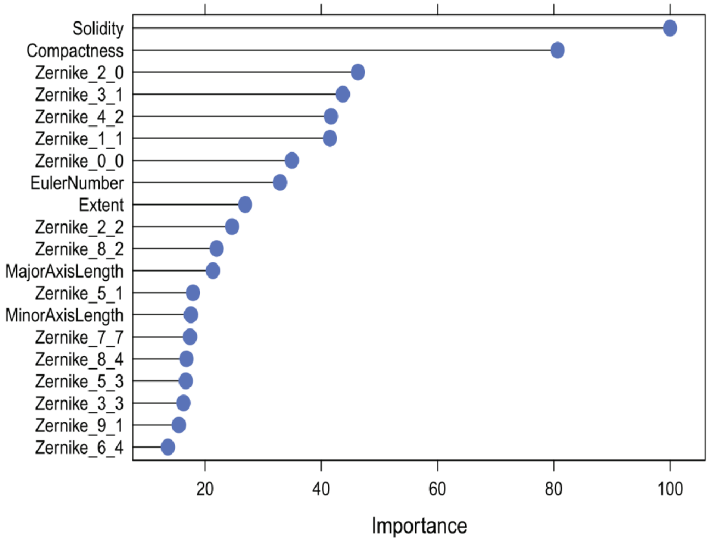

np

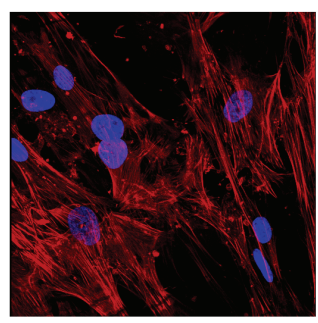

\#4

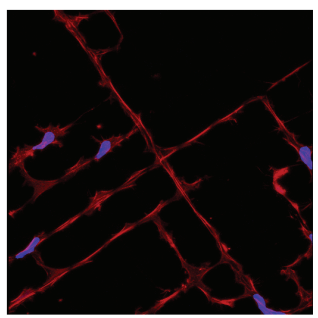

d

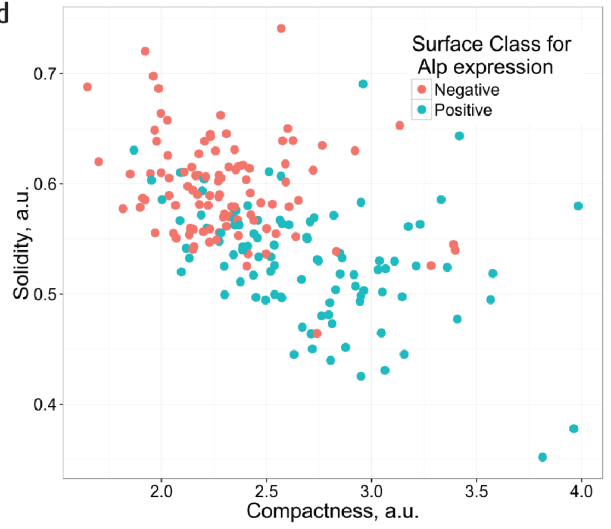

\#8

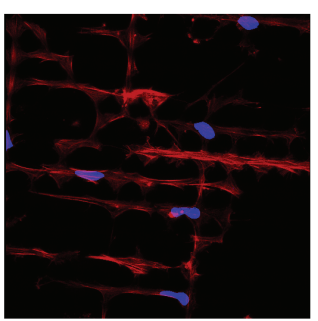

\#7

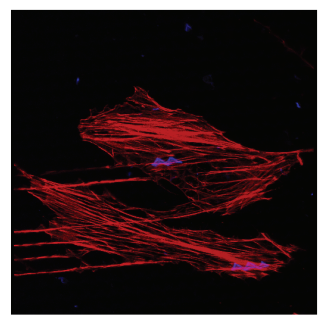

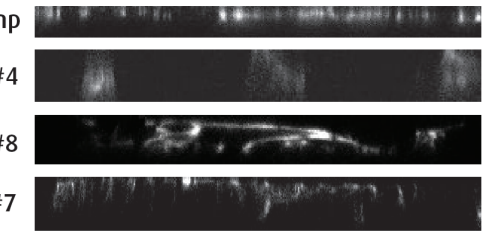

$4.8 \mu \mathrm{m}$

$12 \mu \mathrm{m}$

$9.4 \mu \mathrm{m}$

$6 \mu \mathrm{m}$

Figure 4: Prediction of ALP expression by cell shape. a: Classification Tree for distinguishing negative and positive surfaces by cell shape parameters. b: ROC curve for Random Forest classification algorithm. c: Importance of cell morphology parameters for prediction ALP expression. d: Separation of ALP positive/negative surfaces in 2 most predictive cell shape parameters. e: Confocal images of hMSCS cultured on positive hit topographies \#4, \#8 and negative hit, \#7. Filamentous actin in stained red, nuclei in blue. f: Cross sections of cell on topographies, showing the height of the cells. 
a

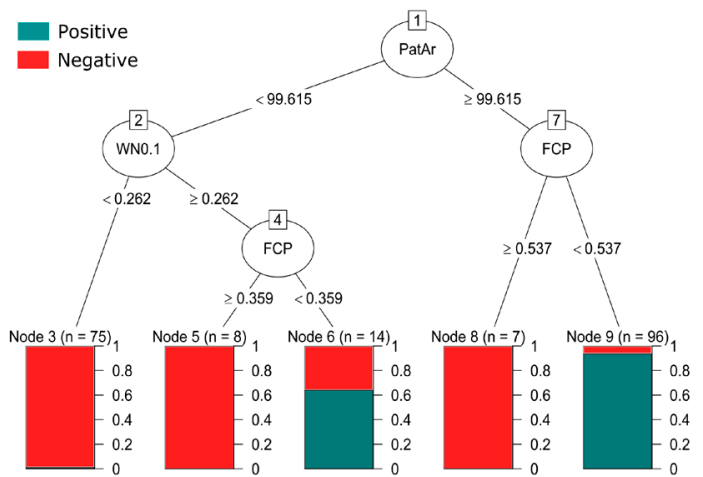

C

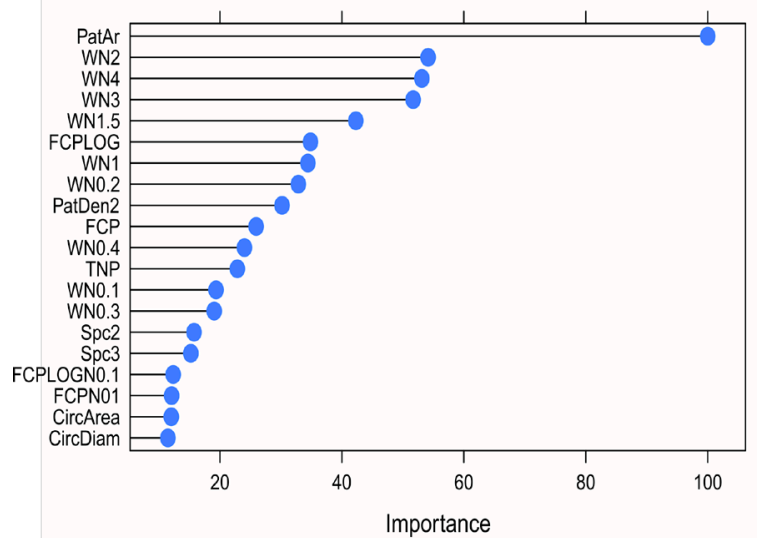

b

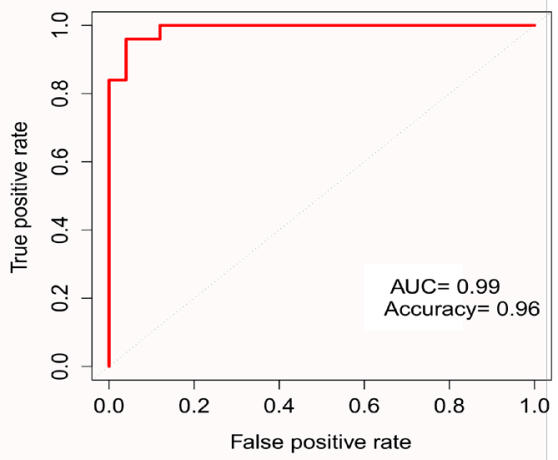

d

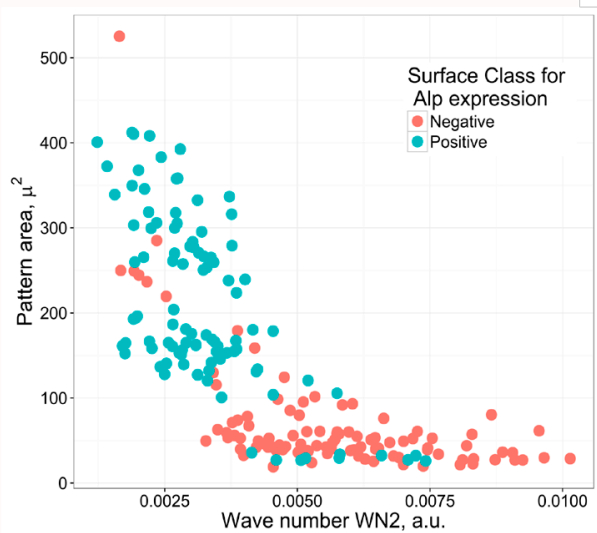

Figure 5: Prediction of ALP expression by surface design a: Classification Tree for distinguishing negative and positive surfaces by surface design parameters. b: ROC curve for Random Forest classification algorithm. c: Importance of surface design parameters for prediction ALP expression. d: Separation of ALP positive/negative surfaces by feature area (PatAr) and Wavenumber 2 (WN2). The two predictive important surface parameters.

Furthermore, to create a robust model, a Random Forest classification analysis was applied. The accuracy of the obtained model was $90 \%$ and the area under the ROC curve was $98 \%$. The obtained model with surface design parameters was extremely accurate (Fig. 5b). From these plots we found that the pattern area, feature density and Wavenumber (WN) parameters are the most relevant features to discriminate surface classes based on ALP expression (Fig. 5c). The WN energy fraction is an abstract number that combines feature shape, size and spacing. The two most important surface features obtained in the model are able to separate the data (Fig. 5d). In this figure it is visible that almost all of the positive hits have a high pattern area (PatAr), confirming our initial visual inspection of the positive features indicating a minimum feature size is required for the cells to be morphologically affected. A combination of the proper pattern area and density were able to induce ALP expression. The WN parameters show that the shape also plays a role but it is not as important as the two previous parameters, which again is in agreement with previous findings that feature size and spacing are more important for inducing osteogenic response than other shape parameters (9). 


\section{Designed topographies instruct differentiation in hMSCs in vitro}

High-throughput screening is a powerful tool to study many parameters in parallel. However, the screening approach usually identifies a fraction of false positives and false negatives. To eliminate these false positive/negative hits, validation experiments have to be performed. To facilitate more conventionally sized in vitro experiments, the hit-topographies, identified with the 290x290 $\mathrm{mm}^{2}$ TopoUnits, were reproduced on discs with a diameter of $1.5 \mathrm{~cm}$ (Fig. 6a). This scaling-up provides the surface area required for validation experiments such as flow cytometry, mineralization and gene expression studies. First, several imaging experiments in which we quantified ALP intensities in the same fashion as the screening experiment were performed to eliminate false positives and false negatives and to identify the most promising topographies. The initial selection was narrowed down to 7 hit-topographies (4 positive, 3 negative, data not shown). To rule out a possible bias introduced by cell size difference appearing in image intensity quantification, ALP expression was also quantified by flow cytometry (Fig. 6b). hMSCs were cultured on 7 titanium hit topographies for 5 days without osteogenic supplements. Tissue culture polystyrene (TCPS) with and without osteogenic medium (medium supplemented with dexamethasone) served as controls. All topographies with high ALP expression have statistically higher percentage of cells positive for ALP (24-28\%) when compared to both the TCPS non-patterned (flat) control (13\%) and the titanium flat control (18\%). Besides, 3 out of 4 ALP-positive surfaces display a similar or higher percentage of ALP positive cells (26-28\%) than the dexamethasone supplemented positive TCPS control (25\%), showing the instructive potential of these topographies in vitro. The negative topographies did not enhance ALP expression, but they did not inhibit ALP expression either. This suggests that perhaps the topography effect is more powerful at amplifying osteogenesis than of inhibiting it.

ALP expression is an early marker for osteogenesis. To investigate the long-term effects of topography, in-vitro mineralization of hMSCs was studied on 3 topographies: two positive \#4, \#8 and one negative \#7. Because orthopedic implant surfaces are exposed to an osteogenic environment when implanted in vivo, this mineralization experiment was performed in osteogenic medium. Tetracyclin was used to fluorescently stain the mineralized matrix of hMSCs which were cultured for 5 weeks on the three topographies (\#4, \#7, \#8) and non-patterned titanium (Fig. 6c). Two topographies, \#4 and \#8 with high AlP expression in earlier experiments, show a 2 to 3 fold increase in mineralized matrix compared to the non-patterned (np) titanium. Topography \#7, which was the highest of the negative hits in the flow cytometry experiment, shows increased mineralization but not statistically significant as determined by ANOVA post hoc testing. To show that the enhanced mineralization induced by the selected topographies is indeed a relevant osteogenic event, gene expression was studied by aPCR (Fig. 6d). RNA was isolated at the 5 week mineralization time point. The osteogenic markers osteocalcin (Ocn), osteopontin (Opn) and bone saleoprotein (Bsp) all showed large differences in gene expression on the positive screening hits (\#4, \#8) of 13-and 8 -fold respectively. The negative hit, \#7, also upregulated the late osteogenic markers significantly, although less so than the positive hits.

In conclusion, the in vitro validation experiments confirmed the osteogenic potential of the positive hit topographies, \#4 and \#8. The negative topography, \#7, showed more intermediate results, in between the positive hits and the non-patterned surface. 


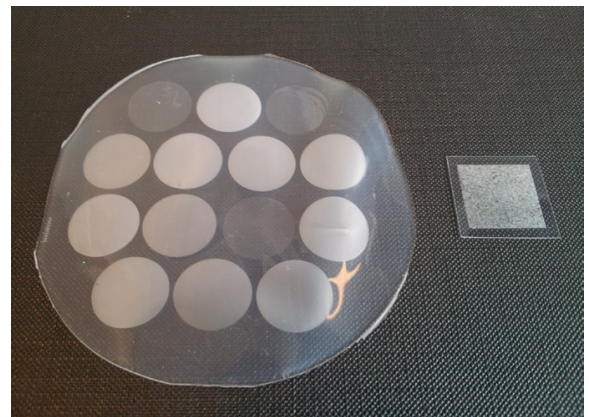

\#4

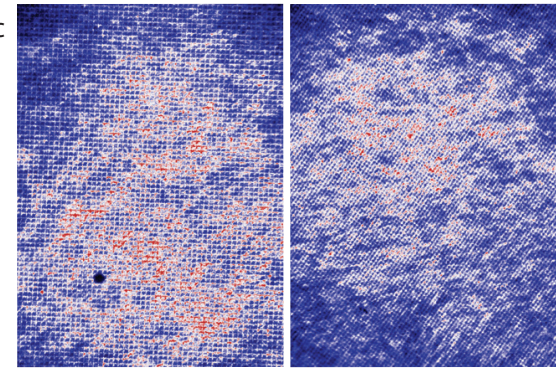

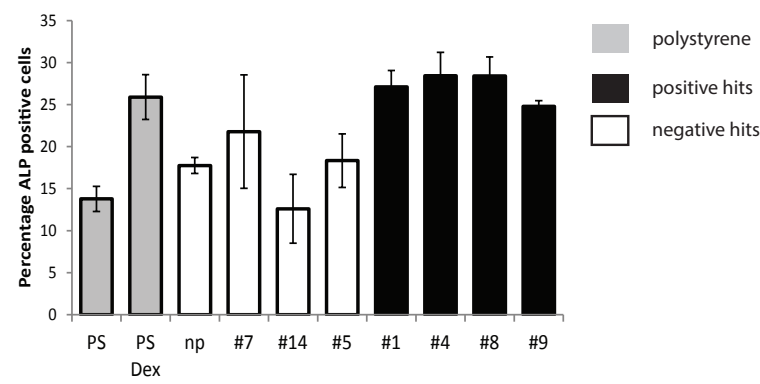

\#7

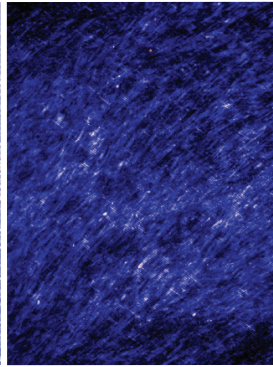

np

np basic

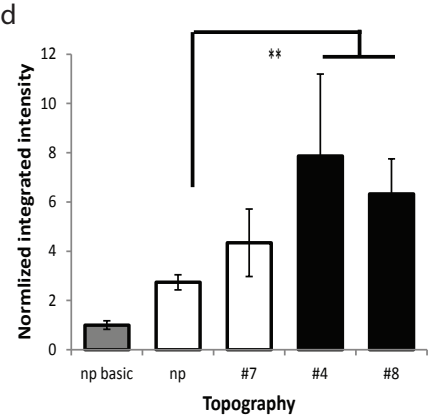

basic medium

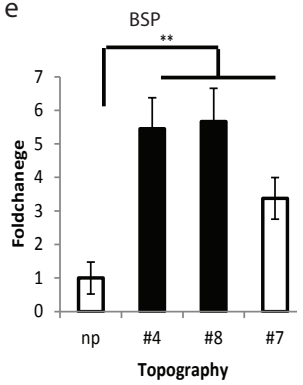

positive hits
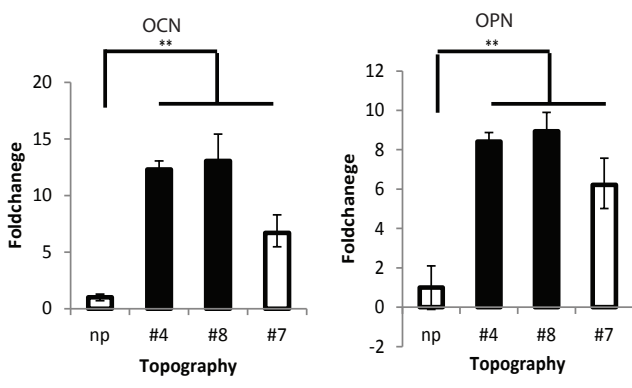

**ANOVA $p<0.05$ as indicated comparison

Error bars are standard deviation

Figure 6: Validation and characterization of Hit Topographies. a: 7 topographies selected from the $2 \times 2 \mathrm{~cm}^{2} \mathrm{chip}$ on the right were applied on a 4 inch silicon wafer in circles with a diameter of $1.5 \mathrm{~cm}$. After hot embossing the resulting discs fit in a 12-wellplate. b: Flow cytometry of hMSCS cultured on hit topographies for 5 days in basic medium. Bars show the mean percentage of ALPpositive cells. ALP-positive cells are defined as those cells expressing surface ALP at a higher median fluorescence intensity relative to the specific isotype control. Means are based on three measurements. c: Representative images of mineralization of hMSCs, visualized after 5 weeks of culture by tetracyclin. d: Integrated intensities were quantified of 15 images from 3 samples for each topography. Bars show mean integrated intensities, error bars show standard deviation. e: Qpcr on hMSCS cultured on hit topographies. Osteopontin (OSP), Osteocalcin (OSN) and Bone saleo protein (BSP) transcripts were quantified after 5 weeks of mineralization. 


\section{Designed topographies improve osseo-integration in vivo}

To confirm that the topographies inducing an osteogenic response of hMSCs in vitro also enhance osseointegration in vivo we performed in vivo experiments using a rabbit model $(27,28)$. In this model experimental implants are fixed into the proximal part of the femur. To this end, three positive hits (\#1, \#4 and \#8), one negative hit (\#5) were included, combined with the non-patterned titanium as a negative control. As a positive control and clinical benchmark one of the best documented surfaces in dental implantology is included: the SLA surface $(29,30)$, which has micro-roughness created by sand-blasting combined with chemical modification through acid etching. All in vitro work was done with titanium-coated PLA. To demonstrate topographical effects in a truly clinically relevant substrate and scale, for the in vivo work, the topographies were fabricated into solid titanium implants using micromachining technologies (Fig. 7a) (see methods). The resulting topographies have high resolution and reproducibility, comparable to the Ti-coated PLA topographies (Fig. 7b). In comparison to the Ti-coated PLA features, the solid Ti features have bigger scallops, caused by the etching process which has a relatively large etching step size of $1 \mu \mathrm{m}$. The implants were surgically inserted into pre-drilled holes created in the femurs of the rabbits. After 4 and 8 weeks the rabbits were sacrificed and the femurs were fixated for pull-out testing and histology.

First, osseo-integration was assessed by pull-out testing, a mechanical test which measures the force required for detachment of the implant (Supplementary Fig. S4a). High pull-out forces correlate with increased fixation to the bone. In all cases, except topography \#1, the pull-out force increases from week 4 to week 8 (Fig. 7c). The measured pull-out force is a good indicator of osseo-integration, which increases over time. The non-patterned negative control has the lowest pull out force at both 4 and 8 weeks after implantation. As expected, any roughness of the implants improves attachment to the bone compared to a very smooth surface. The positive control SLA has higher pull-out force than the smooth surface. Topography \#4 and \#5 have the same pull-out force as the SLA clinical benchmark at the 8 weeks' time-point. One the topographies, \#8 performed a lot better, after 8 weeks, than the SLA surface and was the only surface to perform statistically better than the non-patterned implant.

As a secondary method to assess in vivo response to the topographical implants, the bone-implant contact percentage (\%BIC) was quantified (Fig. 7d). Non-patterned implants have low mean BIC of around 10\% at both 4 weeks and 8. Most non-patterned samples have large areas of fibrous tissue (Fig. 7e). Despite high variation between the animals, the SLA implants and the osteogenic hit topographies, \#1 \#4 \#8, all have a higher BIC at 4 weeks and a significantly higher BIC than the np implant at 8 weeks. Images suggest up to $80 \%$ BIC for these implants at 8 weeks (Fig. 7f). The most striking result is the extremely poor BIC of negative hit topography \#5. Close inspection of the images shows lots of fibrous tissue at both 4 and 8 weeks (Fig. 7g). Additionally, small titanium pieces from the topography can be seen in the tissue close to implant. These pieces have broken off from the implant possibly caused by the high aspect ratio of the rod-like topographies. Clearly, there is limit to the aspect ratio of the microstructures that can be implanted without breakage. Taken together the mechanical testing and BIC assessment show that the osteogenic topographies perform similar to the SLA implants and a lot better than the non-patterned control. 

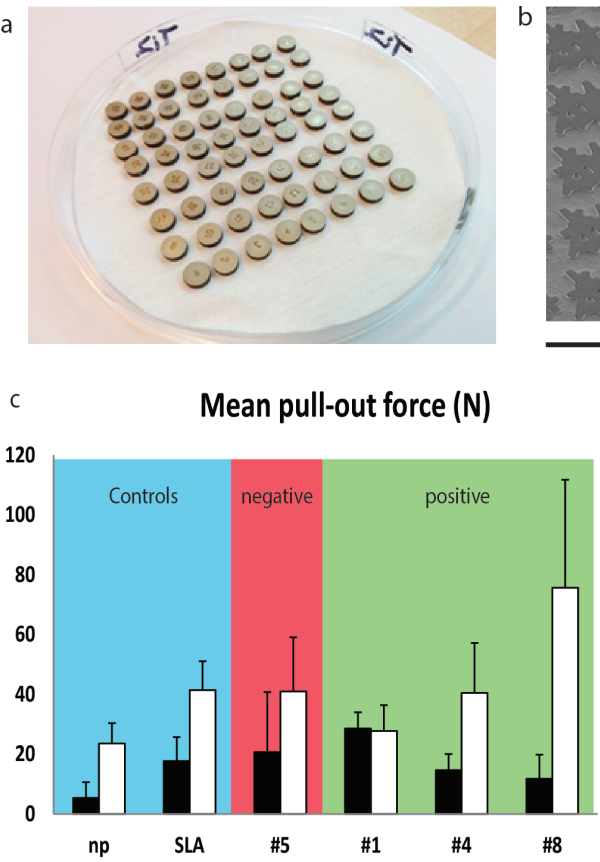

e non-patterned 4 weeks

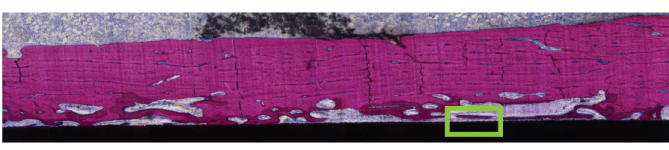

\section{$1 \mathrm{~mm}$}

f SLA 8 weeks

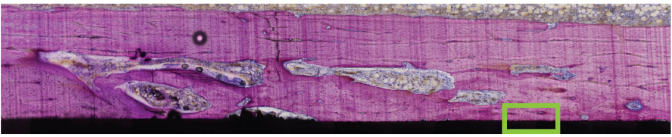

g Topography \#5 4 weeks

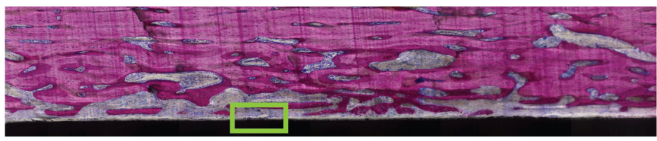

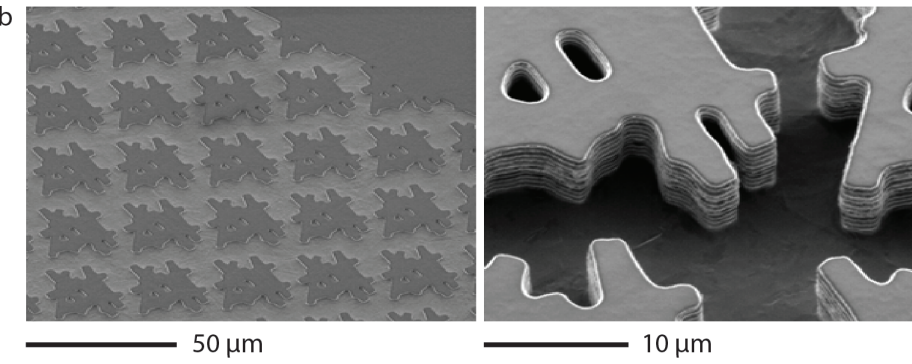

Bone contact \%
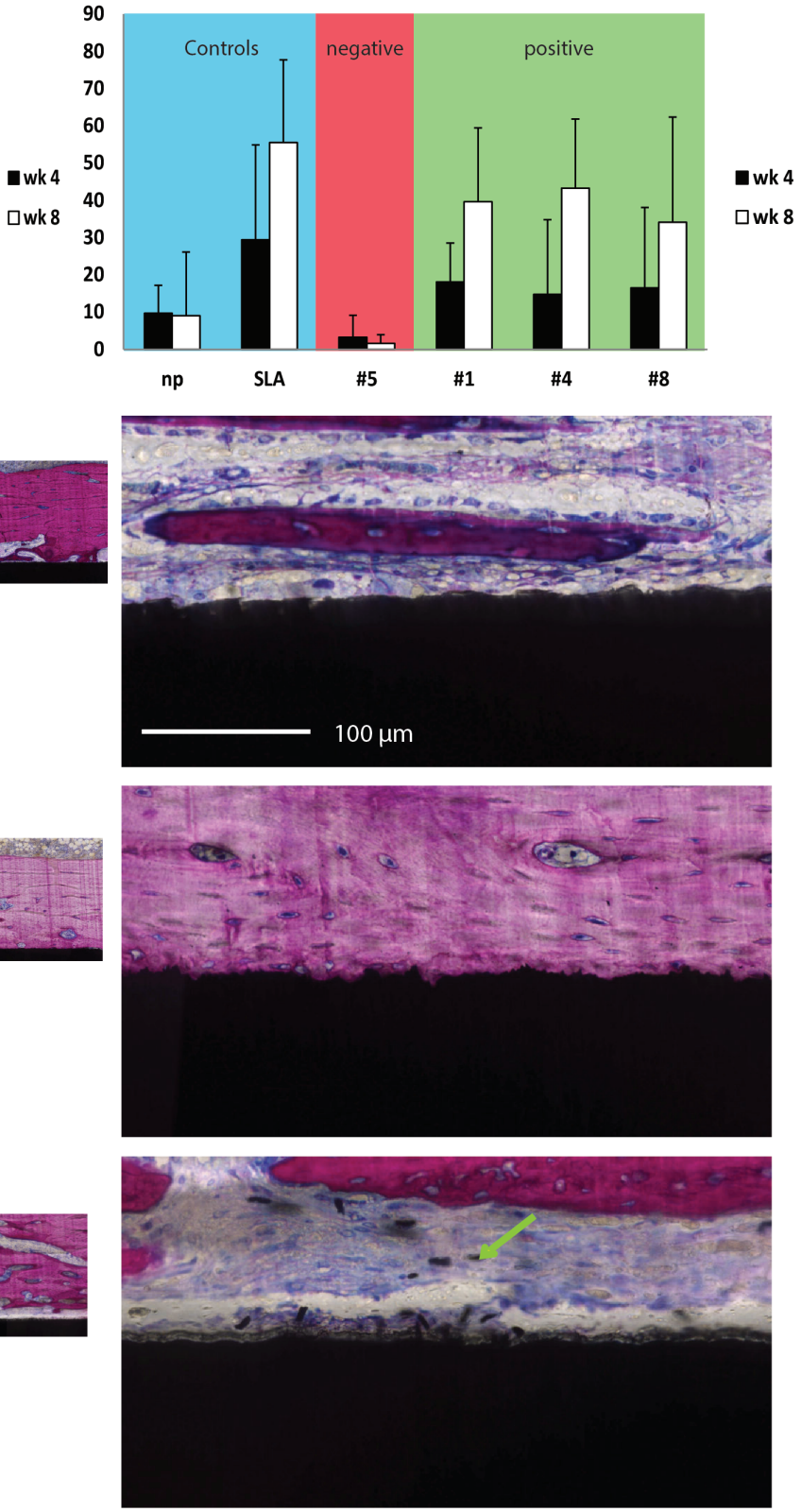
Figure 7: Titanium implant characterization and in vivo results. a: Overview of titanium implants. b: SEM images of micro-machined solid titanium topographies. c: Mean pull-out force $(N)$ of titanium implants. Error bars represent the standard deviation. Controls are non-patterned (nр) as a negative control, and the clinical sand blasted, acid etched benchmark as positive control (SLA). Topography \#5 was a negative hit. The positive hits are \#1, \#4 and \#8. d: Mean Bone-implant contact percentage of the implanted topographies. e: Representative histology images of a bone slice with a non-patterned substrate after 4 weeks of implantation. f: Representative images of a bone slice with a SLA substrate after 8 weeks of implantation. g: Representative images of a bone slice with a \#5 topography substrate after 4 weeks of implantation. $e, f, g$ : On the left there are overviews of the entire width of the implant. On the right there are magnified areas from the implants, indicated with a green box in the left image. A green arrow indicates one of the titanium pieces in the tissue.

\section{Discussion/ Outlook}

Surface topography has significant biological effect on both cells and tissues. For biomedical applications, creating micro roughness with 'non-designed' techniques such as sand-blasting and acid etching can improve clinical outcome (31). Advances in micro-fabrication technology allow precise control over surface topography to the point where surfaces can be covered by specific shapes of defined sizes. HTS is ideal to take advantage of the vast design space provided by new fabrication technologies. Our TopoChip platform enables high-throughput, image based screening of thousands of topographies for cellinstructive effects. A defining feature of the Topochip is its versatility in terms of material choice, enabling the use of a wide variety of clinically relevant biomaterials.

Here, osteogenic topographies identified by the screen showed enhanced in vitro mineralization of hMSCS. Mineralization was confirmed to be an osteogenic effect determined by high upregulation of osteogenic genes OSP, OSC and BSP during the mineralization process. Depending on the assay, the osteogenic topographies perform as good or even slightly better with respect to osseo-integration when compared to the SLA clinical benchmark. In the mechanical tests the positive hit topographies had a similar effect as the SLA surface. The BIC measurements showed high difference between the osteogenic topographies and the negative hit from the screen. The low BIC of the negative hit is probably be due to inflammatory response initiated by small pieces of titanium broken off, as has been reported previously (32). The non-patterned surface is clearly evoking biological responses that are very different from all the topographies. In the HTS experiment it was among the surfaces with the lowest ALP expression. In the in vitro experiments it had the lowest mineralization and expression of osteogenic genes. Finally it performed poorly in the mechanical testing and BIC measurements. It turns out that a completely smooth titanium surface is quite an extreme surface for a cell or a tissue. Literature also reports that microstructured and submicron titanium structured substrates are more osteogenic than flat surfaces in vitro $(33,34)$ and improve outcome in vivo $(35)$. Our positive hit topographies performed equally well as the SLA implant in the mechanical testing and seemed slightly better in the bone contact quantification. However, the SLA implants have a few disadvantages over the designed topographies. Because the sand-blasting is not completely controlled it could create topography with high aspects ratio's that might break of and end up in the tissue as is seen in one of the designed topographies. Additionally, the grit used for the sand-blasting might get embedded in the material and cause cytotoxic effects $(36,37)$. For these reasons it is possible that an improvement of implant stability of designed topographies over the currently used SLA implants will only become apparent after a longer implantation time.

In this work we focused on enabling clinical translation instead of exploring underlying mechanisms. still, our data might provide further insights for existing hypotheses regarding cell-topography 
response mechanisms and mechano-transduction. The dimensions of the topographies proved to be the determining factor for increasing ALP expression. The patterns which prevent flattening of the cells due to confinement by the vertical sides of the features, in which the cells are able to form tube like networks, have the most osteogenic effect. Alignment of hMSCs into more thin elongated cells by micro-grooves increases histone acetylation (38). In addition, Inhibition of histone deacetylases (HDACS) activity was previously shown to increase ALP expression and mineralization of hMSCS (39). Perhaps the osteogenic topographies work through a similar mechanism since the cells on these topographies also have long, thin and aligned morphology. There is some evidence that suggests that calcium dependent Wnt signaling responsible for the effect of topography on titanium, where Wnt5a enhances osteogenesis through a positive feedback with integrins and BMP signaling. (40). YAP/TAZ activity is increased in cells exposed to mechanical stresses, such as stretching, and when exposed to edges and curvatures (41). YAP/TAZ is part of mechano-sensitive signal transduction complex (42) involved in osteogenic differentiation which is thought to be regulated by F-Actin capping proteins. However, YAP/TAZ activity is also strongly dependent on cell-cell contact. Cell-cell contact is minimalized on the osteogenic topographies, while it is probably no longer strongly reduced in fully confluent cell multi-layers which occur during end-point mineralization. The screen was performed under a subconfluent cell density, while the mineralization experiment endpoint was a cell multilayer. This shows that topography influences the cells from sparse to very high densities which suggest a molecular mechanism that is active independent of cell-density.

Osteogenesis was chosen as a biological event to demonstrate the validity of HTS of topographies to improve biomedical implants. This approach is not restricted to osteogenesis, but can be applied to optimize other biomaterial surfaces. A screen has a high chance of success when key components are available: an applicable biomaterial, a relevant cell model and an image-based readout. The screening system is also very suitable to identify optimal topographies for in vitro applications such as specialized cell culture substrates. The Topochip has been used to study macrophage differentiation, stromal immune cell differentiation and thrombocyte activation for vascular applications such as stents and vascular grafts (unpublished data).

This work is a strong demonstration of the power of bio-informatic tools in biomaterial discovery. The model, created from the screening data, was able to predict ALP expression based on topographical parameters with an accuracy of $98 \%$, which is extremely accurate. The cell morphology instructed by the feature dimensions correlate with ALP expression. Topographical feature parameters are able to induce ALP expression in a highly predictable manner. The predictive power of the feature shape was much stronger than the cell morphology. This could be due to imperfections of image segmentation or it is because we lack the right morphology descriptors. Information of the feature design parametercell response relationship, provided by our approach, offers the possibility to use iterative rounds of screening to further optimize biomaterial surfaces. Ultimately, the use of bioinformatics to analyze cell-response to large libraries of topographies should enable us to decipher the topographical 'language' to instruct cells. Even though we expect this topographical language to be very cell and material specific it will offer significant new insights to mechano-biology. The osteogenic topographies that were identified in this work will serve as model substrates for transcriptomics to investigate the underlying mechano-biological mechanisms of the osteogenic response.

In conclusion, this work clearly demonstrated that it is indeed feasible to identify biologically relevant biomaterial properties in a screening fashion that makes use of miniaturized experimental conditions and up-scale these to clinically relevant size and materials. In this way it shows the value of HTS in biomaterials, also known as Materiomics (43). Our data suggests that the topographies have both 
short term and long term osteogenic effects. Further studies will be required to look into the underlying mechanisms. This work is a strong first example of HTS of biomaterials that yields data on preclinical efficacy and serves as a proof of principle for future studies.

\section{Materials and methods}

\section{Fabrication}

\section{Topochip Design and fabrication.}

The in silico design of the Topochip is generated using an algorithm that combines the primitive shapes: triangle, circle and rectangle to generate complex shapes, as described (19). The in silico design was used to create a chromium mask for photolithography. The micro patterns were etched from the silicon wafer, by directional reactive ion etching (DRIE), generating a silicon master mould for hot embossing. To facilitate demoulding, the master was coated with a 'non-sticky' layer of perfluorodecyltrichlorosilane (FOTS) (ABCR, AB111155). The master was subsequently used for hot embossing of PLA films (250 $\mathrm{mm}$ thick) (Folienwerk Wolfen $\mathrm{GmbH}$ ) using an Obducat nanoimprint device. Imprinting was carried out at $120^{\circ} \mathrm{C}$ with 30 bars of pressure for $10 \mathrm{~min}$. Demoulding took place after cooling down to $80^{\circ} \mathrm{C}$. The PLA TopoChips and up-scaled surfaces were coated with $200 \mathrm{~nm}$ Titanium by sputter coating at a sputtering rate of $6 \mathrm{~nm} /$ minute.

\section{Titanium implant fabrication}

The Ti implants were fabricated using micromachining technologies. An outline of the fabrication scheme is illustrated in supplementary Figure S3a. Annealed Ti plates of $99.6 \%$ purity of $150 \mathrm{~mm} \times 150 \mathrm{~mm}$ and thickness of $2 \mathrm{~mm}$ were bought from Goodfellow, UK. (A) Ti plates were first cut by laser into $100 \mathrm{~mm}$ diameter substrates to adapt to the $100 \mathrm{~mm}$ cleanroom facility in the nanolab of MESA+ Institute of Nanotechnology. After a pre-polish step using polishing paste, the Ti substrates were final polished to achieve a roughness of about $10 \mathrm{~nm}$ by chemical mechanical polishing (CMP Mecapol E460, Cabot Semisperse 25 slurry : $\mathrm{H}_{2} \mathrm{O}=1: 2$ ) for about $60 \mathrm{~min}$. (B) After ultrasonic cleaning in demi (DI) water for $60 \mathrm{~min}$, in acetone for $30 \mathrm{~min}$ and in isopropanol (IPA) for $30 \mathrm{~min}, 780 \mathrm{~nm} \mathrm{SiO}$ x was deposited by plasma enhanced chemical vapor deposition (PECVD, Oxford Plasmalab system 80). (C) The lithography step was performed by using positive photoresist OIR 908-12 (Arch Chemical, Inc). After exposure (EVG 620) and development (OPD 4262, Arch Chemical, Inc), the Ti substrates were hard baked on a hot plate at $120^{\circ} \mathrm{C}$ for $30 \mathrm{~min}$. (D) The $\mathrm{SiO}_{x}$ layer was etched by directional reactive ion etching (DRIE, Adixen AMS $100 \mathrm{DE}$ ) for 2 min at a $\mathrm{C}_{4} \mathrm{~F}_{8}$ flow of $20 \mathrm{sccm}$, He flow of $150 \mathrm{sccm}, \mathrm{CH}_{4}$ flow of $15 \mathrm{sccm}$, inductively coupled plasma (ICP) power of 2800 $\mathrm{W}$, capacitively coupled plasma (CCP) power of $350 \mathrm{~W}$ and at a temperature of $-10^{\circ} \mathrm{C}$. After stripping the photoresist in Oxygen $\left(\mathrm{O}_{2}\right.$ ) plasma (Tepla 300E), (F) the Ti substrates were etched using an alternation of an etching step of the etchant combination of $\mathrm{Cl}_{2} / \mathrm{BCl}_{3} / \mathrm{Ar}$ plasma and an oxidation step by $\mathrm{O}_{2}$ plasma (Oxford Plasmalab system 100). The etching step was performed at an etchant $\mathrm{Cl}_{2} / \mathrm{BCl}_{3} / \mathrm{Ar}$ flow of 50/50/20 sccm, ICP of $2000 \mathrm{~W}$, CCP of $100 \mathrm{~W}$ and pressure of 10 mTorr. The oxidation step was performed at an $\mathrm{O}_{2}$ flow of $30 \mathrm{sccm}$, ICP of $500 \mathrm{~W}$, CCP of $15 \mathrm{~W}$ and pressure of 33 mTorr. An alternation of $45 \mathrm{sec}$ etching time and $15 \mathrm{sec}$ oxidation time results in a Ti etch rate of $1 \mu \mathrm{m} / \mathrm{min}$ and therefore 10 cycles were performed to receive a structure depth of about $10 \mu \mathrm{m}$. The substrate temperature was kept at $50^{\circ} \mathrm{C}$ for both steps. $(\mathrm{G})$ The $\mathrm{SiO}_{\mathrm{x}}$ masking layer was removed in 2 min using the same method as in step (D). 
Individual Ti samples were labelled by laser from the backside of the substrates and subsequently cut out by electric discharge machining (EDM, Charmilles, Switzerland). The fabricated Ti substrates were coated beforehand with AZ 9260 resist for surface protection. Polytetrafluoroethylene (PTFE) caps were designed and fabricated CNC machining to avoid interlocking from lateral bone attachment as well as for the easiness of fixture attachment for tensile testing. The illustrations of a Ti implant with its PTFE cover are shown in supplementary Figure S2b. To obtain a tight fit of the PTFE cover to the Ti implant, the PTFE cover has an outer diameter of $7.05 \mathrm{~mm}$, wall thickness of $0.4 \mathrm{~mm}$ and inner height of $1.90 \mathrm{~mm}$. A through hole of $4 \mathrm{~mm}$ diameter was made for fixture fixation during tensile test.

\section{In vitro experiments}

\section{Cell culture}

HMSCS were cultured in basic medium consisting of oxMEM (Gibco) supplemented with 10\% fetal bovine serum (Lonza), $2 \mathrm{mM}$ I-glutamine (Gibco), $0.2 \mathrm{mM}$ ascorbic acid (Sigma Aldrich) and $100 \mathrm{U} / \mathrm{ml}$ penicillin + $100 \mathrm{~g} / \mathrm{ml}$ streptomycin (Gibco). Osteogenic medium contains basic medium with $10^{-8} \mathrm{M}$ dexamethasone (Sigma Aldrich) and $0.01 \mathrm{M}$ ß-glycerol phosphate (Sigma Aldrich). Adipogenic consisted of DMEM (Gibco) supplemented with $100 \mathrm{U} / \mathrm{ml}$ penicillin $+100 \mu \mathrm{g} / \mathrm{ml}$ streptomycin (Gibco), 10\% foetal bovine serum (Lonza), $0.2 \mathrm{mM}$ Indomethacin (Sigma Aldrich), $0.5 \mathrm{mM}$ IBMX (Sigma Aldrich,) $10^{-6} \mathrm{M}$ dexamethasone (Sigma Aldrich) and $10 \mu \mathrm{g} / \mathrm{ml}$ Insulin (human, Sigma Aldrich). In all experiments, medium was refreshed every two days.

\section{Topochip staining and imaging}

Prior to seeding, titanium-coated Topochips are wetted for a minimum of 2 days in basic medium at $37^{\circ} \mathrm{C}$ and at in a $5 \% \mathrm{CO}_{2}$ atmosphere; wetting is prolonged due to hydrophobic properties of patterned surfaces. For the screening experiment eight Topochips were seeded with passage 4 hMSCs (Lonza) at a density of 3000 cells $/ \mathrm{cm} 2$. After 5 days of culture in basic medium, the cells were washed in phosphate buffered (PBS, Sigma Aldrich) and fixated in 70\% ethanol and stained for ALP, cell nuclei and cytoskeleton. After fixation the cells were incubated with blocking buffer, consisting of 2\% bovine serum albumin (BSA, Sigma Aldrich) in PBS for 30 minutes followed by primary antibody incubation of 2 h with 1:50 anti-ALP (sc137213, Santa Cruz Biotech) in blocking buffer. After washing with PBS 4 times, the cells were incubated with 1:100 Goat-anti-mouse-Alexa594 (Life Technologies) in blocking buffer for $1 \mathrm{~h}$, followed by 4 further PBS washings and incubation of 1:100 Phalloidin-Alexa488 (Life Technologies) in blocking buffer. Next, after washing with PBS 4 times nuclear staining with 1:10000 4',6-Diamidino-2-phenylindole (Dapi, Life technologies) was done. Finally the Topochips were mounted on coverslips with Mowiol 4-88 (Sigma Aldrich) after washing with PBS for two times.

After sample drying the chips were imaged using a BD Pathway 435 automated fluorescence microscope. A total of 4356 images (one for each TopoUnit) were acquired for each chip and fluorescence channel. All images were combined into a large montage of 62,964 by 62,964 pixels (3964 megapixels). 


\section{Image analysis}

Image analysis is described in detail elsewhere (20). In summary, custom matlab scripts were used for flat-field correction, image normalization and background subtraction. Quality control features were calculated and used to detect outliers. From the large montage each original image was cropped into separate images of $800 \times 800$ pixels. For each of the TopoUnit images, Cell Profiler (26) was used to obtain the cell morphology. To prevent influence of the walls on the cell morphology measurements, cell touching the walls were filtered out of the dataset. The DAPI channel was used to recognize nuclei, using the Otsu Adaptive thresholding method. Subsequently, each of these nuclei was used to initiate the search for a corresponding cell, by making use of the phalloidin-Alexa488 staining by Otsu adaptive thresholding and the provided propagation algorithm. Measurements were performed on the resulting shapes. Within the defined individual cell areas, ALP intensities were determined by measuring the maximum ALP intensity, integrated ALP intensity and integrated ALP intensity corrected for actin intensity. Hit rankings were made by using the Mann-Whitney $U$ test.

\section{Statistical analysis}

To be able to identify the surface design parameters that can influence ALP expression, we selected 100 surfaces with the highest ALP expression and 100 surfaces with the lowest ALP expression. To classify topographies that had a positive or negative effect on the Integrated Intensity of the ALP signal, we used classification trees algorithms from "party" package (44) implemented in R ver. 3.1 .2 (45). The classification tree was visualized by this "party" package (46). To further create a robust predictive model we assessed the behaviour of multiple machine learning algorithms (data not shown) and selected the Random Forest algorithm (47) that gave the highest accuracy on the tested data set. In order to have a training set for testing accuracy of the model, data set was split in to 2 parts. The first part contained $3 / 4$ of the data and was used for model training and the remaining $1 / 4$ was used for model testing. Models were trained with 10 fold cross validation in the "caret" package (48).

\section{Flow cytometry}

HMSCS were seeded at a density of 3500 cells $/ \mathrm{cm}^{2}$ on enlarged surfaces of the selected patterns and cultured for 5 days in basic hMSC medium without osteogenesis-stimulating compounds. As a control to the patterned surfaces, cells were also cultivated on non-patterned (flat) surfaces in basic hMSc medium with or without dexamethasone. The medium was refreshed every 2 days. Following the cell culture period, the cells were harvested and stained with an anti-human ALP antibody that was then labeled with a Phycoerythrin (PE)-labeled specific secondary antibody. Flow cytometry was performed immediately after staining using a FACSCalibur (BD Biosciences) and its CellQuest software (BD Biosciences).

\section{Tetracyclin staining.}

After 5 weeks of minteralizaion in osteogenic medium, 1\% teatrcyclin was added to the medium for 24 hours before fixation in 10\% formalin (Sigma). 5 images from each sample, with 3 samples for each topography were imaged with a BD Pathway 435 automated fluorescence microscope. Integrated intensities were quantified using imagej software (49). 


\section{QPCR}

Total RNA was isolated by using a RNA isolation kit (Bioline) according to manufacturer's protocol. $500 \mathrm{ng}$ of RNA was used to prepare cDNA according to manufacturer's instructions in a $20 \mu$ reaction volume (Biorad). Quantitative RT-QPCR was performed in a $20 \mu$ reaction volume with $0.01 \mathrm{nmol}$ of of forward and reverse primers. 40 reaction cycles were performed which consisted of annealing at $60^{\circ} \mathrm{C}$ for 15 seconds and extension at $72^{\circ} \mathrm{C}$ for 15 seconds. Glyceraldehyde 3-phosphate dehydrogenase (GAPDH) was used as a house-keeping gene and relative expression were determined using $\Delta \Delta C T$ method.

\section{In vivo experiments}

\section{Animal surgery}

A total of 24 New Zealand White female rabbits from Dossy Biotechnology Ltd, Chengdu, China, were used. The weight of the animals was between 3000 and $3500 \mathrm{~g}$. Housing of the animals and surgery was performed at Dossy Biotechnology LtD, Chengdu, China. Animals were housed individually in cages in an environment with a temperature $16-28^{\circ} \mathrm{C}$ and a humidity of $45-70 \%$. Animals were fed ad libitum and they had free to access water. Animals were observed per the standard monitoring schedule at Dossy Biotechnology. Before surgery Ti implants were ultrasonically cleaned in acetone for $1 \mathrm{~h}$ and by IPA for $1 \mathrm{~h}$. Energy-dispersive $\mathrm{X}$-ray spectroscopy (EDX) measurement was performed to ensure that implant surface was free from impurities and therefore safe for implantation.

All procedures were approved by the local ethic committee for the use of animals for studies and performed under general anesthesia and sterile conditions. The rabbits were sedated by intravenous application of pentobarbital sodium (3,0 mg/kg body weight).

Operation sites were shaved and sterilized with iodine and 70\% EtOH and the animal was covered with a sterile blanket. Surgery was performed as described earlier $(27,28)$ and illustrated in the supplementary Figure 54 . In short, a $5 \mathrm{~cm}$ incision on the proximal part of the femur was made, penetrating the epidermis, dermis and fascial layers exposing the underlying periosteum. An additional medial-anterior incision was made through the periosteum. The periosteum was elevated with a periosteal elevator. Two holes were made with a $1.0 \mathrm{~mm}$ twist drill (Stryker, Germany), using a custom made drill guide. With a custom made $7.05 \mathrm{~mm}$ diameter bur, a stable leveled site for the implants was made using the same drill guide. The implants were kept in place by a mesh plate (Endomed, The Netherlands). The subcutaneous layers were repositioned and layer by layer sutured with 4-0 silk suture. The surgical site was sterilized with iodine and animals were then placed in their cages and observed until awake and mobile. Wound healing was monitored daily. Rabbits were euthanized with an overdose pentobarbital sodium (>3.0 mg/kg body weight). After euthanasia the femur with implant was removed and cleared from surrounding tissues. Bones with samples were then fixated in 10\% neutral buffered formalin (>72 h) and kept at 70\% EtOH for further experiments. No incidents were registered during the course of animal experiments. 


\section{Tensile test}

The tensile test was performed using mechanical pull bench Adamel-Lhomargy DY-32 installed with a calibrated Sandoo SH-200 load cell of 0-200 N. PMMA cylinders were fabricated and fixed to the Ti implants using Cyanoacrylate glue, see supplementary Figure S5. A through hole was drilled in the PMMA cylinder for connecting a $0.038 \mathrm{~mm}$ wire to the upper clamp. The pulling force was applied at $1 \mathrm{~mm} / \mathrm{min}$ until the implant was removed from the bone.

\section{Histology methods}

Fixated samples were dehydrated in ethanol series and embedded in Methyl methacrylate (MMA). Sections were processed on a histological diamond saw (Leica SP1600) and stained with 1\% Methylene Blue and 0,3\% Basic Fuchsin solution. Three sections across the middle were made for each implant and scanned with a histologic slide scanner (Nanozoomer, Hamamatsu). For all sections the boneimplant contact percentage (\%BIC) was measured. This is defined as all areas with direct bone contact to the implant without gaps or fibrous tissue. All samples were assessed by a blinded observer. To avoid confounding of the data, random measurements from the dataset were verified by two additional independent observers.

\section{Acknowledgements}

JdB, DS and FH gratefully acknowledge the financial support of the NanoNext NL initiative. JdB acknowledges the financial support of the Dutch province of Limburg, AV the European Union's Seventh Framework Program (FP7/2007-2013) (grant agreement 289720).YZ thanks Peter Linders and Ite-Jan Hoolsema from the Nanolab. Materiomics BV acknowledges Sip Jan Boorsma, Tom Buitenhuis, Peter Post, Andre Eppingbroek and Roy Kooijman, Techno Centrum voor Onderwijs en Onderzoek, University of Twente, Enschede and Harry Homan and Andre from Abbring, IMDS, Roden. 


\section{Supplementary figures.}

basic medium + ßGP

Mineralization Alizarin red, 5 weeks

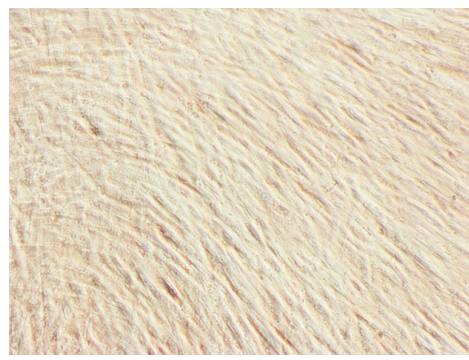

basic medium $+\beta G P$

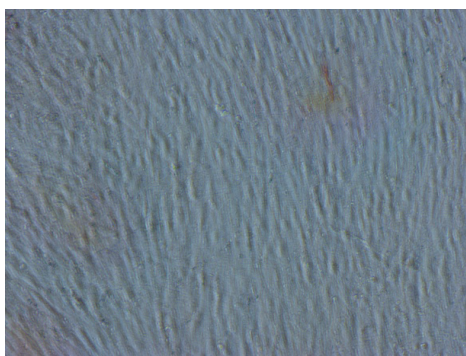

Adipogenesis

Oil red O

3 weeks osteogenic medium

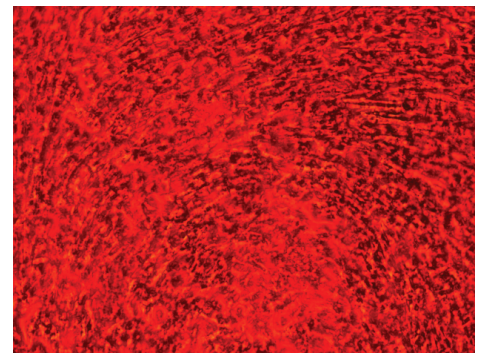

adipogenic medium

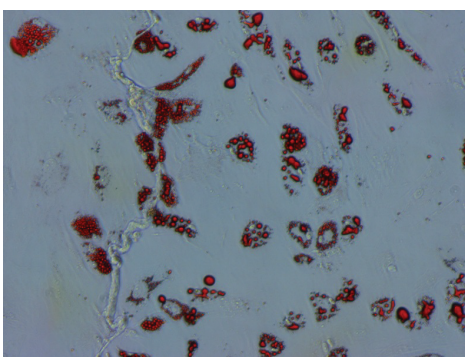

Figure S1: hMSC lineage potential test

a: hMSC differentiation into the osteogenic lineage was tested with a alizarin red mineralization assay. b: hMSC differentiation into the adipogenic lineage was tested with a oil red o staining of lipid vesicles. 

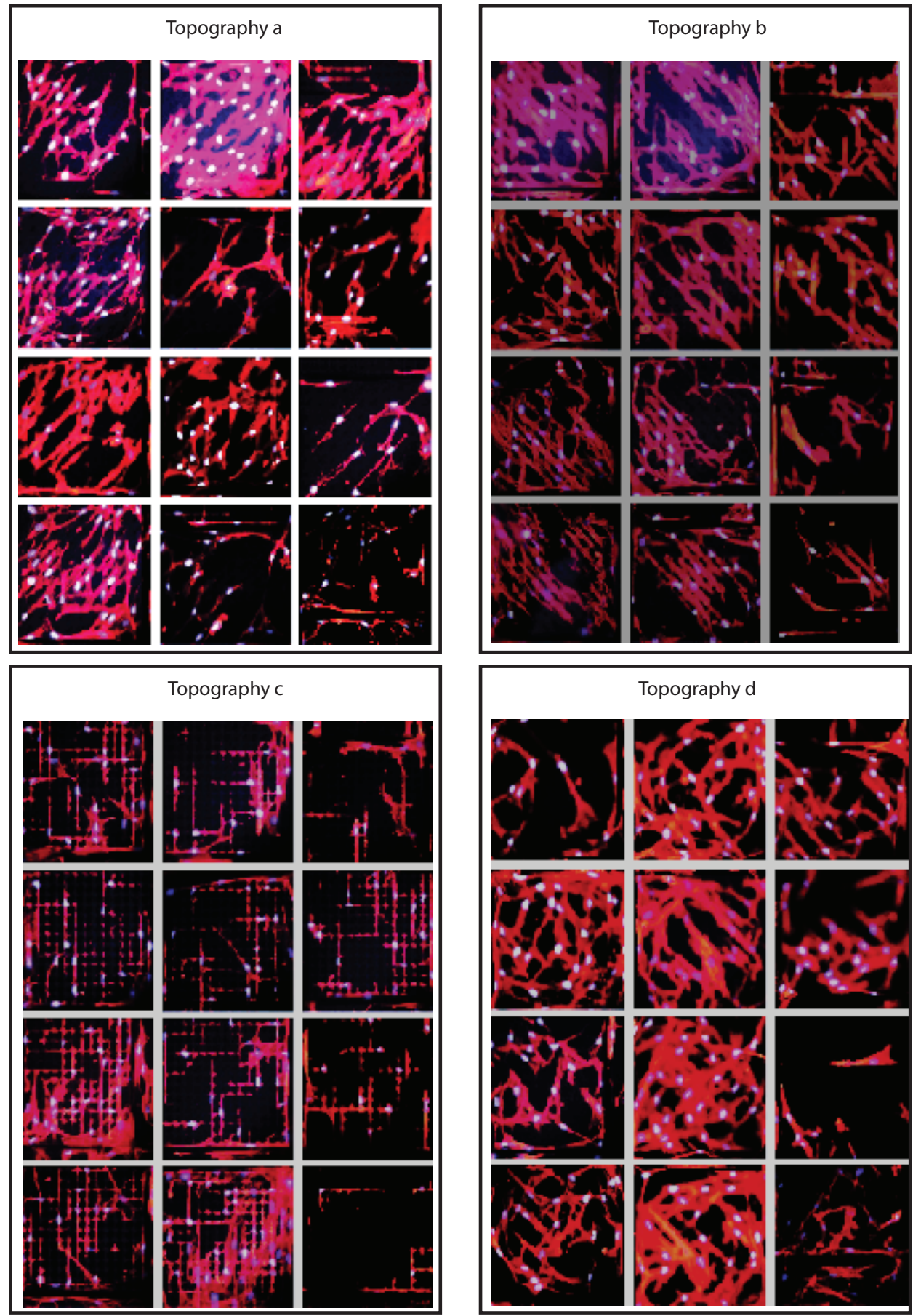

\section{Figure S2: Screen reproducibility}

Images of 4 random TopoUnits a, b, c, d, from the Topochip screen are shown together to demonstrate cell morphology reproducibility between Topochips within the screening experiment. Actin is stained red and the nuclei are light blue. 
a

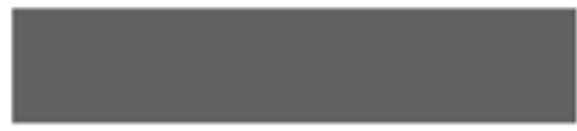

A

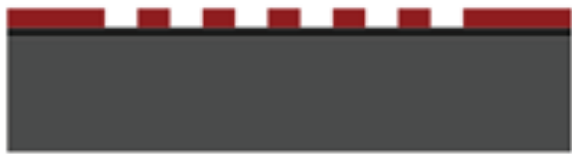

C

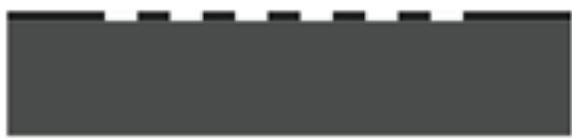

E

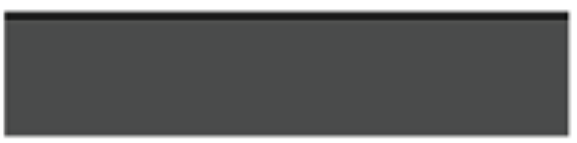

B

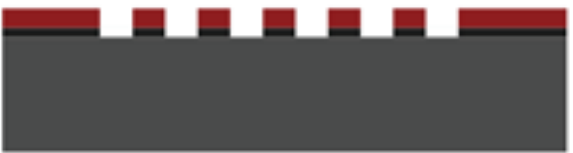

D

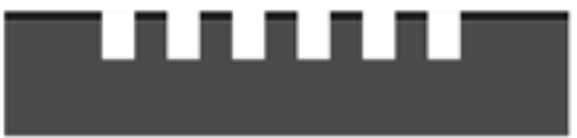

$\mathbf{F}$

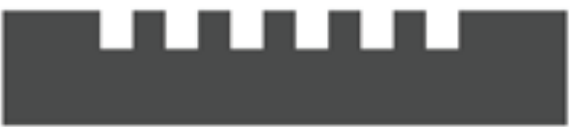

G

$\mathrm{SiO}$

b

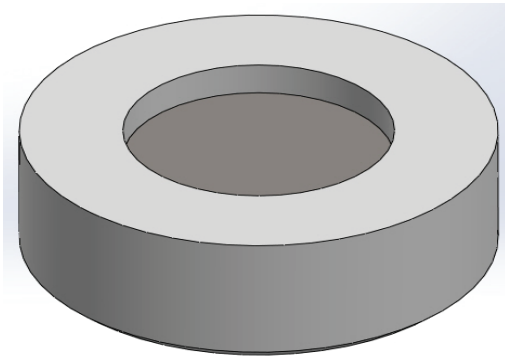

$\mathrm{Ti}$

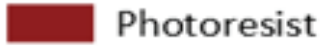

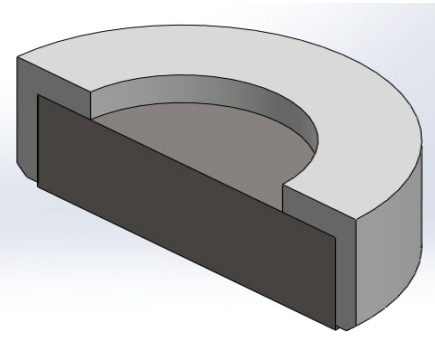

Figure S3: Titanium bone implant fabrication

a: Ti implants fabrication scheme (see materials and methods for full explanation): A: Ti plates were first cut by laser into $100 \mathrm{~mm}$ diameter substrates; B: $780 \mathrm{~nm} \mathrm{SiO}_{x}$ was deposited by plasma enhanced chemical vapor deposition; C: Lithography step was performed by using positive photoresist; $D$ : The SiO ${ }_{x}$ layer was etched by directional reactive ion etching; E: Stripping the photoresist in Oxygen plasma; $\mathrm{F}$ : Ti substrates were etched using an alternation of an etching step of the etchant combination of $\mathrm{Cl}_{2} / \mathrm{BCl}_{3} / \mathrm{Ar}$ plasma and an oxidation step by $\mathrm{O}_{2}$ G: The SiO ${ }_{x}$ masking layer was removed in 2 min using the same method as in step (D).

b: Illustrations of Ti implants and its PTFE cover. 


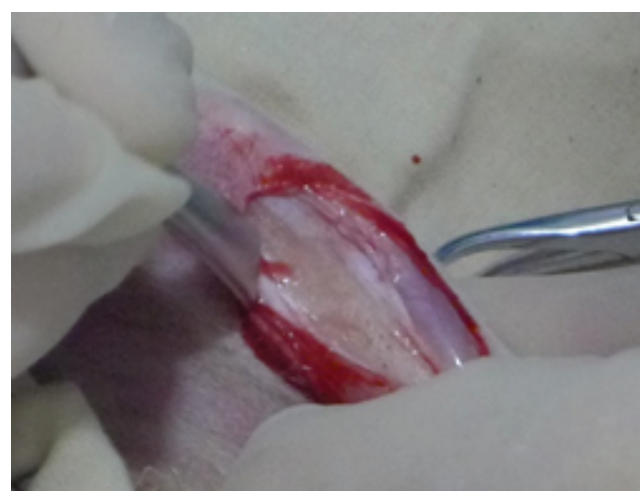

c

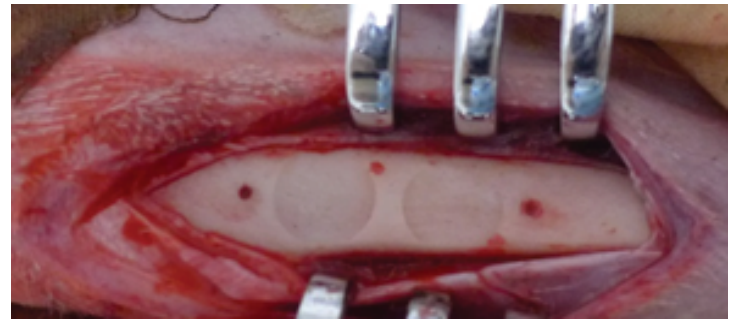

\section{Figure S4: Animal surgery}

a: $5 \mathrm{~cm}$ incision was made for the implantation site. With help of a custom made guide (b) the leveled platforms and 2 holes for fixation of the mesh plate were made (c). The samples covered with Teflon caps are kept in place with the mesh plate (d). b

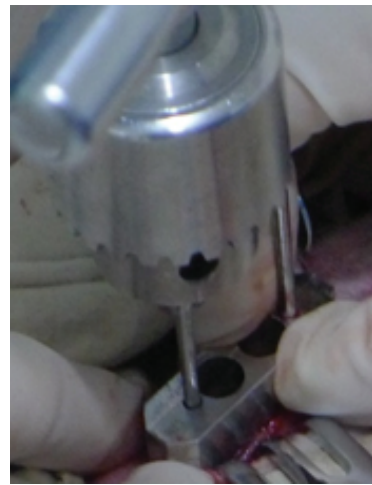

$\mathrm{d}$

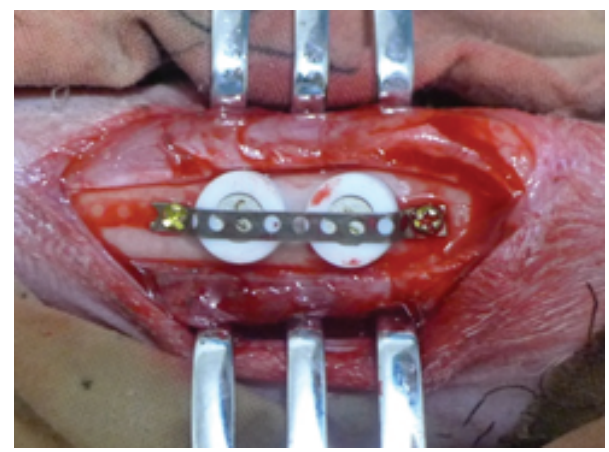

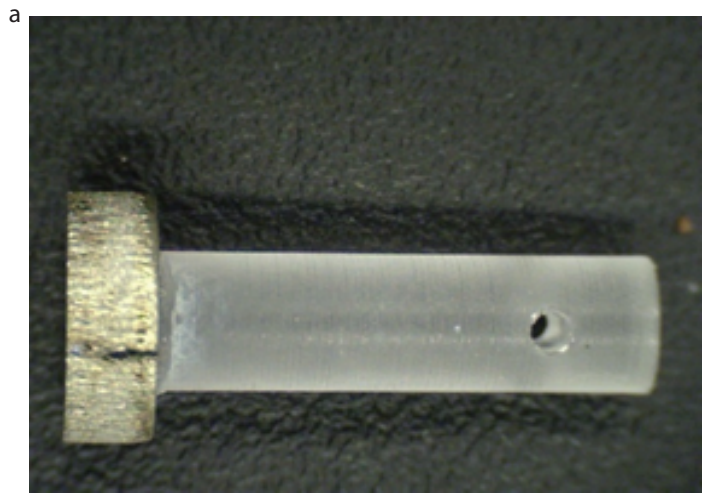

Figure S5: Pull out testing set-up

a: A PMMA cylinder glued to a Ti implant; b: A rabbit femur with Ti implants fixed to a support and clamping jig. 


\section{Literature}

1. Busscher HJ, van der Mei HC, Subbiahdoss G, Jutte PC, van den Dungen JJ, Zaat SA, et al. Biomaterial-associated infection: locating the finish line in the race for the surface. Sci Transl Med. 2012;4(153).

2. Goodheart JR, Miller MA, Mann KA. In vivo loss of cement-bone interlock reduces fixation strength in total knee arthroplasties. Journal of orthopaedic research : official publication of the Orthopaedic Research Society. 2014;32(8):1052-60.

3. Buth J, Laheij RJ. Early complications and endoleaks after endovascular abdominal aortic aneurysm repair: report of a multicenter study. J Vasc Surg. 2000;31(1 Pt 1):134-46.

4. Dalby MJ, Gadegaard N, Tare R, Andar A, Riehle MO, Herzyk P, et al. The control of human mesenchymal cell differentiation using nanoscale symmetry and disorder. Nature materials. 2007 Dec;6(12):997-1003. PubMed PMID: 17891143. Epub 2007/09/25. eng.

5. Song S, Kim EJ, Bahney (S, Miclau T, Marcucio R, Roy S. The synergistic effect of microtopography and biochemical culture environment to promote angiogenesis and osteogenic differentiation of human mesenchymal stem cells. Acta biomaterialia. 2015 Feb 28. PubMed PMID: 25735800. Epub 2015/03/05. Eng.

6. Tan KK, Tann JY, Sathe SR, Goh SH, Ma D, Goh EL, et al. Enhanced differentiation of neural progenitor cells into neurons of the mesencephalic dopaminergic subtype on topographical patterns. Biomaterials. 2015 Mar;43:32-43. PubMed PMID: 25591959. Epub 2015/01/17. eng.

7. Justesen J, Lorentzen M, Andersen LK, Hansen O, Chevallier J, Modin C, et al. Spatial and temporal changes in the morphology of preosteoblastic cells seeded on microstructured tantalum surfaces. Journal of biomedical materials research Part A. 2009 Jun 15;89(4):885-94. PubMed PMID: 18465820. Epub 2008/05/10. eng.

8. Kolind K, Dolatshahi-Pirouz A, Lovmand J, Pedersen FS, Foss M, Besenbacher F. A combinatorial screening of human fibroblast responses on microstructured surfaces. Biomaterials. 2010 Dec;31(35):9182-91. PubMed PMID: 20832853. Epub 2010/09/14. eng.

9. Lovmand J, Justesen J, Foss M, Lauridsen RH, Lovmand M, Modin C, et al. The use of combinatorial topographical libraries for the screening of enhanced osteogenic expression and mineralization. Biomaterials. 2009 Apr;30(11):2015-22. PubMed PMID: 19178942. Epub 2009/01/31. eng.

10. Li W, Tang QY, Jadhav AD, Narang A, Qian WX, Shi P, et al. Large-scale Topographical Screen for Investigation of Physical Neural-Guidance Cues. Scientific reports. 2015;5:8644. PubMed PMID: 25728549. Epub 2015/03/03. eng.

11. Ratcliffe A. Difficulties in the translation of functionalized biomaterials into regenerative medicine clinical products. Biomaterials. 2011;32(18):4215-7.

12. Feller $L$, Jadwat $Y$, Khammissa RA, Meyerov R, Schechter I, Lemmer J. Cellular Responses Evoked by Different Surface Characteristics of Intraosseous Titanium Implants. Biomed Res Int. 2015;2015:171945. PubMed PMID: 25767803. Pubmed Central PMCID: PMC4341860. Epub 2015/03/15. Eng.

13. Schwartz Z, Lohmann CH, Oefinger J, Bonewald LF, Dean DD, Boyan BD. Implant surface characteristics modulate differentiation behavior of cells in the osteoblastic lineage. Adv Dent Res. 1999 Jun;13:38-48. PubMed PMID: 11276745. Epub 2001/03/30. eng.

14. Arlin ML. Survival and success of sandblasted, large-grit, acid-etched and titanium plasma-sprayed implants: a retrospective study. Journal (Canadian Dental Association). 2007 Nov;73(9):821. PubMed PMID: 18028757. Epub 2007/11/22. eng.

15. Mei Y, Saha K, Bogatyrev SR, Yang J, Hook AL, Kalcioglu Zl, et al. Combinatorial development of biomaterials for clonal growth of human pluripotent stem cells. Nature materials. 2010 Sep;9(9):768-78. PubMed PMID: 20729850. Pubmed Central PMCID: PMC3388774. Epub 2010/08/24. eng.

16. Ranga A, Gobaa S, Okawa Y, Mosiewicz K, Negro A, Lutolf MP. 3D niche microarrays for systems-level analyses of cell fate. Nature communications. 2014;5:4324. PubMed PMID: 25027775. Pubmed Central PMCID: PMC4104440. Epub 2014/07/17. eng.

17. Gobaa S, Hoehnel S, Roccio M, Negro A, Kobel S, Lutolf MP. Artificial niche microarrays for probing single stem cell fate in high throughput. Nature methods. 2011 Nov;8(11):949-55. PubMed PMID: 21983923. Epub 2011/10/11. eng. 
18. Khan F, Tare RS, Kanczler JM, Oreffo RO, Bradley M. Strategies for cell manipulation and skeletal tissue engineering using high-throughput polymer blend formulation and microarray techniques. Biomaterials. 2010 Mar;31(8):2216-28. PubMed PMID: 20056271. Epub 2010/01/09. eng.

19. Unadkat HV, Hulsman M, Cornelissen K, Papenburg BJ, Truckenmuller RK, Carpenter AE, et al. An algorithm-based topographical biomaterials library to instruct cell fate. Proceedings of the National Academy of Sciences of the United States of America. 2011 Oct 4;108(40):16565-70. PubMed PMID: 21949368. Pubmed Central PMCID: 3189082. Epub 2011/09/29. eng.

20. Hulsman M, Hulshof F, Unadkat H, Papenburg BJ, Stamatialis DF, Truckenmuller R, et al. Analysis of highthroughput screening reveals the effect of surface topographies on cellular morphology. Acta biomaterialia. 2015 Mar;15:29-38. PubMed PMID: 25554402. Epub 2015/01/03. eng.

21. Pittenger MF, Mackay AM, Beck SC, Jaiswal RK, Douglas R, Mosca JD, et al. Multilineage potential of adult human mesenchymal stem cells. Science. 1999 Apr 2;284(5411):143-7. PubMed PMID: 10102814. Epub 1999/04/02. eng.

22. Bianco P, Robey PG. Stem cells in tissue engineering. Nature. 2001 Nov 1;414(6859):118-21. PubMed PMID: 11689957. Epub 2001/11/02. eng.

23. Yuan H, Fernandes H, Habibovic P, de Boer J, Barradas AMC, de Ruiter A, et al. Osteoinductive ceramics as a synthetic alternative to autologous bone grafting. Proceedings of the National Academy of Sciences. 2010 August 3, 2010;107(31):13614-9.

24. Crane GM, Ishaug SL, Mikos AG. Bone tissue engineering. Nat Med. 1995 Dec;1(12):1322-4. PubMed PMID: 7489417. Epub 1995/12/01. eng.

25. Hessle L, Johnson KA, Anderson HC, Narisawa S, Sali A, Goding JW, et al. Tissue-nonspecific alkaline phosphatase and plasma cell membrane glycoprotein-1 are central antagonistic regulators of bone mineralization. Proceedings of the National Academy of Sciences of the United States of America. 2002;99(14):9445-9.

26. Carpenter AE, Jones TR, Lamprecht MR, Clarke C, Kang IH, Friman 0, et al. CellProfiler: image analysis software for identifying and quantifying cell phenotypes. Genome biology. 2006;7(10):31.

27. Ronold HJ, Ellingsen JE. The use of a coin shaped implant for direct in situ measurement of attachment strength for osseointegrating biomaterial surfaces. Biomaterials. 2002;23(10):2201-9.

28. Monjo M, Lamolle SF, Lyngstadaas SP, Ronold HJ, Ellingsen JE. In vivo expression of osteogenic markers and bone mineral density at the surface of fluoride-modified titanium implants. Biomaterials. 2008;29(28):3771-80.

29. Sennerby L, Persson LG, Berglundh T, Wennerberg A, Lindhe J. Implant stability during initiation and resolution of experimental periimplantitis: an experimental study in the dog. Clin Implant Dent Relat Res. 2005;7(3):136-40.

30. Roccuzzo M, De Angelis N, Bonino L, Aglietta M. Ten-year results of a three-arm prospective cohort study on implants in periodontally compromised patients. Part 1: implant loss and radiographic bone loss. Clinical Oral Implants Research. 2010;21(5):490-6.

31. Mendonca G, Mendonca DB, Aragao FJ, Cooper LF. Advancing dental implant surface technology- from micron- to nanotopography. Biomaterials. 2008;29(28):3822-35.

32. Chen W, Li Z, Guo Y, Zhou Y, Zhang Z, Zhang Y, et al. Wear particles promote reactive oxygen species-mediated inflammation via the nicotinamide adenine dinucleotide phosphate oxidase pathway in macrophages surrounding loosened implants. Cellular physiology and biochemistry : international journal of experimental cellular physiology, biochemistry, and pharmacology. 2015;35(5):1857-67. PubMed PMID: 25871260. Epub 2015/04/15. eng.

33. Olivares-Navarrete R, Hyzy SL, Hutton DL, Erdman CP, Wieland M, Boyan BD, et al. Direct and indirect effects of microstructured titanium substrates on the induction of mesenchymal stem cell differentiation towards the osteoblast lineage. Biomaterials. 2010 Apr;31(10):2728-35. PubMed PMID: 20053436. Pubmed Central PMCID: PMC2821717. Epub 2010/01/08. eng.

34. Zhao G, Raines AL, Wieland M, Schwartz Z, Boyan BD. Requirement for both micron- and submicron scale structure for synergistic responses of osteoblasts to substrate surface energy and topography. Biomaterials. 2007 
Jun;28(18):2821-9. PubMed PMID: 17368532. Pubmed Central PMCID: PMC2754822. Epub 2007/03/21. eng.

35. Bornstein MM, Harnisch H, Lussi A, Buser D. Clinical performance of wide-body implants with a sandblasted and acid-etched (SLA) surface: results of a 3-year follow-up study in a referral clinic. The International journal of oral \& maxillofacial implants. 2007 Jul-Aug;22(4):631-8. PubMed PMID: 17929525. Epub 2007/10/13. eng.

36. Darvell BW, Samman N, Luk WK, Clark RK, Tideman H. Contamination of titanium castings by aluminium oxide blasting. Journal of dentistry. 1995 Oct;23(5):319-22. PubMed PMID: 7560379. Epub 1995/10/01. eng.

37. Martin DC, O'Ryan FS, Indresano AT, Bogdanos P, Wang B, Hui RL, et al. Characteristics of implant failures in patients with a history of oral bisphosphonate therapy. Journal of oral and maxillofacial surgery: official journal of the American Association of Oral and Maxillofacial Surgeons. 2010 Mar;68(3):508-14. PubMed PMID: 20171469. Epub 2010/02/23. eng.

38. Li Y, Chu JS, Kurpinski K, Li X, Bautista DM, Yang L, et al. Biophysical regulation of histone acetylation in mesenchymal stem cells. Biophysical journal. 2011 Apr 20;100(8):1902-9. PubMed PMID: 21504726. Pubmed Central PMCID: PMC3077706. Epub 2011/04/21. eng.

39. de Boer J, Licht R, Bongers M, van der Klundert T, Arends R, van Blitterswijk C. Inhibition of histone acetylation as a tool in bone tissue engineering. Tissue engineering. 2006 Oct;12(10):2927-37. PubMed PMID: 17518660. Epub 2007/05/24. eng.

40. Olivares-Navarrete R, Hyzy SL, Park JH, Dunn GR, Haithcock DA, Wasilewski CE, et al. Mediation of osteogenic differentiation of human mesenchymal stem cells on titanium surfaces by a Wnt-integrin feedback loop. Biomaterials. 2011 Sep;32(27):6399-411. PubMed PMID: 21636130. Pubmed Central PMCID: PMC3350791. Epub 2011/06/04. eng.

41. Aragona M, Panciera T, Manfrin A, Giulitti S, Michielin F, Elvassore N, et al. A mechanical checkpoint controls multicellular growth through YAP/TAZ regulation by actin-processing factors. Cell. 2013;154(5):1047-59.

42. Dupont S, Morsut L, Aragona M, Enzo E, Giulitti S, Cordenonsi M, et al. Role of YAP/TAZ in mechanotransduction. Nature. 2011;474(7350):179-83.

43. Cranford S, Buehler MJ. Materiomics: biological protein materials, from nano to macro. Nanotechnol Sci Appl. 2010;3:127-48. PubMed PMID: 24198478. Pubmed Central PMCID: PMC3781696. Epub 2010/01/01. eng.

44. Therneau T. rpart: Recursive Partitioning and Regression Trees. 2014.

45. Team. RC. R: A language and environment for statistical computing. R Foundation for Statistical Computing, Vienna, Austria. URL http://www.R-project.org/.(2014). 2014.

46. Hothorn T, Hornik K, Zeileis A. Unbiased recursive partitioning: A conditional inference framework. Journal of Computational and Graphical Statistics. 2006 Sep;15(3):651-74. PubMed PMID: ISI:000240705500009. English.

47. Wiener ALaM. Classification and Regression by randomForest. RNews 2002 ( 2(3)):18-22.

48. Kuhn. M. caret: Classification and Regression Training R package version 6.0-37. http://CRAN.R-project.org/ package=caret. 2014

49. Abramoff MD MP, Ram S). Image processing with Imagej. Biophotonics International. 2004 (11):36-42. 


\section{CHAPTER 4}

\section{DESIGNED POLYSTYRENE MICROTOPOGRAPHIES INHIBIT OSTEOGENIC AND ADIPOGENIC DIFFERENTIATION OF HMSCS}

Frits Hulshof ${ }^{12}$, Jan de Boer ${ }^{1}$, Dimitrios Stamatialis ${ }^{2}$

1Department of cell biology inspired tissue engineering, MERLN Institute for Technology-Inspired Regenerative Medicine, University of Maastricht, The Netherlands

2Department of Biomaterials Science and Technology, MIRA Institute for Biomedical Technology and Technical Medicine, University of Twente, Enschede, The Netherlands 


\section{Abstract}

Biomaterial surface properties such as surface chemistry, stiffness and topography influence cell response. In the case of surface topography it is well established that submicron and microstructures, are able to elicit biological responses in various cells. Studies into topography effects on cells use many different materials, most often based on available production methods. In this work we study the instructive effect of Polystyrene (PS) microtopographies on differentiation of hMSCs. Our results show that the microtopographies are able to strongly inhibit osteogenesis by lowering ALP expression and mineralization. Additionally, adipogenesis is inhibited by several microtopography designs, depending on spacing of the features. Cell counting only showed minor differences between non-patterned and patterned areas indicating that the inhibition of differentiation is probably not caused by differences in cell density. Finally, when comparing the differentiation of hMSC cultured on PS topographies to hMSCs cultured on the exact same titanium topographies the contrasting outcomes demonstrate the importance of substrate material choice in these studies.

\section{Introduction}

Medical devices and cell culture substrates can be improved by optimizing cell-biomaterial interactions which are governed by properties such as chemistry, elasticity and topography. In order to do this we have to understand the underlying mechanisms that operate cell response to these individual biomaterial properties. Tailoring surface topography to instruct cell response has been the topic of many studies. Topographical cues can have significant effects such as the potential to direct differentiation of neural progenitor cells (1), Human mesenchymal stromal cells $(\mathrm{hMSCS})(2,3)$ and induced pluripotent stem cells (IPS) (4).

To study topography, many different substrate materials are used. This biomaterial choice is usually determined by the available fabrication methods which were often originally developed for creating microelectromechanical systems (MEMS) and microfluidics, such as silicon and PDMS. Ideally the material choice should be made based on the possibility of applying the topographies on an eventual application, such as titanium (Ti) as a model substrate to modify Ti bone implants. There is increasing evidence that topographically induced effects on one material do not necessarily occur on another material.

In this work we aim to study the effect of biomechanical cues of microtopographies fabricated on polystyrene (PS). Even though PS is difficult to process due to its high stiffness and brittleness it is still the golden standard for tissue culture substrates due to its low cost and excellent optical properties for microscopy. The possibility to compare experimental data from experiments with PS topographies to the large amounts of historical data generated with conventional PS tissue culture plastics will be valuable.

Our previous work has identified a set of designed microtopographies, fabricated in Ti that are able to modulate osteogenic differentiation of hMSCs (Chapter 3). From these, seven topographies were selected here for fabrication in PS. These topographies have a range of size, shape and spacing that influence the morphology of hMSCs in varying ways. The PS topographies were fabricated by hot embossing with specialized Ormostamp ${ }^{\circledR}$ moulds.

The instructive potential of the microtopographies is investigated by observing phenotypical outcomes of hMSC differentiation $(5,6)$. The hMSCs are used as a model system to study topography effects on cells because they are known to be mechano-sensitive, meaning they respond to biomaterial mechanical properties and surface topography and because hMSC osteogenic and adipogenic differentiation are esta- 
blished readouts. In addition, hMSCs show functional response to topography at the nanometer (2), submicron (7) and micrometer-scale (8). As differentiation outcomes, osteogenesis and adipogenesis are investigated. Finally we compare the instructive potential of the PS topographies to the Ti topographies to demonstrate the importance of substrate material choice to study topographical effects.

\section{Materials and methods}

\section{Polystyrene topography fabrication}

Seven Bioactive microtopographies were selected (\# 1 to \#7) from an in-silico library as previously described (9). Polystyrene (PS) topographies were prepared by hot embossing a PS film (Goodfellow, USA) with specialized moulds, as described in (Appendix 1). In summary, the inverse structure of the topographies is produced on silicon by standard photo lithography and deep reactive etching. The silicon mould is used to make a positive mould in poly(dimethylsiloxane) (PDMS). The PDMS mould is required to create a second negative mould in OrmoStamp ${ }^{\circledR}$ hybrid polymer (micro resist technology Gmbh) which serves as the mould for hot embossing of the PS films. The fabrication of the Ormostamp mould is necessary since demoulding PS from silicon is highly problematic and often leads to destruction of the mould or the imprint. Hot embossing was performed with the Obducat T-Nil system with the following conditions: imprinting at $140^{\circ} \mathrm{C}$ for 5 min at 10 Bar, with a demoulding temperature of $80^{\circ} \mathrm{C}$. Before cell culture, the PS topographies were briefly treated with a mild oxygen plasma to improve cell adhesion. Plasma treatment was performed for $30 \mathrm{~s}$ at $75 \mathrm{mTor}, 50 \mathrm{sccm} \mathrm{02,50} \mathrm{W.}$

\section{Titanium coated PLA topography fabrication.}

The micro patterns were etched from the silicon wafer, by directional reactive ion etching (DRIE), generating a silicon master mould for hot embossing. To facilitate demoulding, the master was coated with a 'non-adhesive' layer of perfluorodecyltrichlorosilane (FDTS) (ABCR, AB111155). The master was subsequently used for hot embossing of PLA films (250 $\mu \mathrm{m}$ thick) (Folienwerk Wolfen GmbH) using an obducat nanoimprint device. Imprinting was carried out at $120^{\circ} \mathrm{C}$ with 30 bars of pressure for 10 min. Demoulding took place after cooling down to $80^{\circ} \mathrm{C}$. The PLA TopoChips and up-scaled surfaces were coated with $200 \mathrm{~nm}$ Titanium by sputter coating at a sputtering rate of $6 \mathrm{~nm} /$ minute.

\section{Cell culture}

HMSCs were cultured in basic medium consisting of oxMEM (Gibco) suplemented with 10\% fetal bovine serum (Lonza), $2 \mathrm{mM}$ I-glutamine (Gibco), $0.2 \mathrm{mM}$ ascorbic acid (Sigma Aldrich) and $100 \mathrm{U} / \mathrm{ml}$ penicillin + $100 \mathrm{~g} / \mathrm{ml}$ streptomycin (Gibco). Osteogenic medium contains basic medium with 10-8 $\mathrm{M}$ dexamethasone (Sigma Aldrich) and 0.01 M ß-glycerol phosphate (Sigma Aldrich). Adipogenic consisted of DMEM (Gibco) supplemented with $100 \mathrm{U} / \mathrm{ml}$ penicillin $+100 \mathrm{mg} / \mathrm{ml}$ streptomycin (Gibco), 10\% foetal bovine serum (Lonza), $0.2 \mathrm{mM}$ Indomethacin (Sigma Aldrich), $0.5 \mathrm{mM}$ IBMX (Sigma Aldrich,)10-6 M dexamethasone (Sigma Aldrich) and $10 \mathrm{mg} / \mathrm{ml}$ Insulin (human, Sigma Aldrich). In all experiments, medium was refreshed every two days. 


\section{Cell counting with DAPI}

At the designated time points the samples were fixated for 10 mins in 10\% formalin (Sigma Aldrich). After washing the cells 3 times with PBS they were stained with 1:10000 4',6-diamidino-2-phenylindole (DAPI, from Life Technologies) for 20 mins. After washing the cells 3 more times with PBS they were imaged in bright field and with a DAPI filter set using an EVOS microscope. The bright field images were used to determine the micro-patterned and non-patterned areas. The DAPI channel was used to count the nuclei. For every topography 4 ratios were determined from 3 fields of view by dividing the amount of cells in the non-patterned area by the amount of cells in the microstructured area.

\section{Alizarin red staining}

After 5 weeks of culture the samples were fixated for 20 mins in 10\% formalin (Sigma Aldrich) and washed twice with demi water. Subsequently, the cells were covered by a freshly filtered 2\% aqueous alizarin red (Sigma Aldrich) solution (pH 4.2) for 10 mins. After washing of excess stain with demi water the cells were imaged with a Nikon E600 microscope.

\section{Oil red 0}

After 3 weeks of culture the samples were fixated for 30 mins in 10\% formalin (Sigma Aldrich. After rinsing with demi water followed a 5 min incubation by with 60\% isopropanol and $5 \mathrm{~min}$ in $0.22 \mathrm{\mu M}$ Oil Red 0 solution. After staining, the cells were rinsed with demiwater and imaged with a Nikon E600 microscope. If direct visual inspection was not possible, the Oil Red 0 staining was quantified by extraction with 4\% Igepal (Sigma Aldrich) in isopropanol for 15 mins. Absorption of the extract was measured at $540 \mathrm{~nm}$ using a Thermo Scientific Multiscan 60 platereader and normalized to the positive control (non-patterned polystyrene).

\section{Ca assay}

The calcium assays (BioAssay Systems) were performed according to manufacturer's protocol. Absorption was measured using a Thermo Scientific Multiscan 60 platereader.

\section{Flow cytometry}

HMSCs were seeded at a density of 3500 cells $/ \mathrm{cm}^{2}$ on up-scaled surfaces of three selected patterns and cultured for 5 days in basic hMSC medium without osteogenesis-stimulating compounds. As a control to the patterned surfaces, cells were also cultivated on non-patterned (np) surfaces in basic hMsc medium with or without dexamethasone. The medium was refreshed every 2 days. Following the cell culture period, the cells were harvested and stained with an anti-human ALP antibody that was then labeled with a Phycoerythrin (PE)-labeled specific secondary antibody. Flow cytometry was performed immediately after staining using a FACSCalibur (BD Biosciences) and its CellQuest software (BD Biosciences). 


\section{Results}

To investigate the effect of microtopography and to be able to compare the results to the experimental data available from experiments with standard PS tissue culture plates, a selection of 7 Topographies which are able to modulate hMSC osteogenesis was fabricated in PS films. Differentiation of hMSCs was chosen as a model system to investigate cell response to topography because the availability of functional readouts and the sensitivity of hMSCs to the biophysical signals induced by surface topography.

First, early osteogenic differentiation of hMSCs was assessed by quantifying the amount of tissue nonspecific alkaline phosphatase (ALP) positive cells cultured on the PS topographies after 6 days of culture in medium without osteogenic supplements (Fig. 1a). ALP is a commonly used marker for osteogenic differentiation as it is crucial for mineralization of bone because it generates the inorganic phosphate essential for hydroxyapatite crystallization (10). When cultured in basic medium, 11\% of hMSCs were positive for ALP. Adding dexamethasone increased the percentage of ALP positive cells to $18 \%$. In stark contrast to earlier findings with titanium coated topographies (Chapter 3 ), all the polystyrene topographies strongly down regulated ALP expression with ALP positive percentages that ranges between 1\% and 3\%. Polystyrene microtopographies seem to inhibit hMSCs differentiation, indicated by a down regulation of osteogenic marker expression.

Next, osteogenic differentiation was investigated at a late time point. For this, the PS topographies were used in a 5 week mineralization experiment with hMSCs and the degree of mineralization was assessed by Alizarin red staining (Fig. 1b). Both non-patterned controls (either the bottom of a polystyrene well-plate (+) or a piece of flat polystyrene film (np), which is used for topography fabrication) showed strong mineralization, indicated by the deep red staining. In the negative control, a nonpatterned well plate with hMSCs cultured in basic medium (-), we did not find mineralization. In contrast to the osteogenic titanium topographies the hMSCS cultured on the PS topographies showed very little staining, indicating that the surface topographies very strongly inhibit mineralization. To confirm this remarkable finding, the experiment was repeated with larger PS circular films which contains several smaller circular areas with topographies (Fig. 1c). The circular areas are covered with the topographical patterns, while the area in the middle and between the patterned areas is non-patterned. In agreement with the previous experiment it is clearly visible by the red staining that the non-patterned area is mineralized, while areas with the topographical patterns are not. Closer inspection by microscopy showed that there is actually a sharp boundary between mineralized hMSCs and non-mineralized hMSCs which coincided to the non-patterned and patterned areas respectively (Fig. 1d). These results were confirmed with a calcium assay (Fig. 1e). Total calcium levels on the micro patterned PS were at least three times lower on the topographies, compared to the nonpatterned surface. 
a

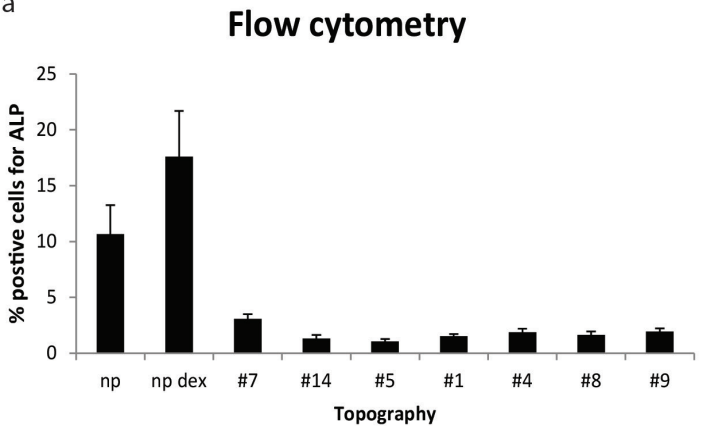

C

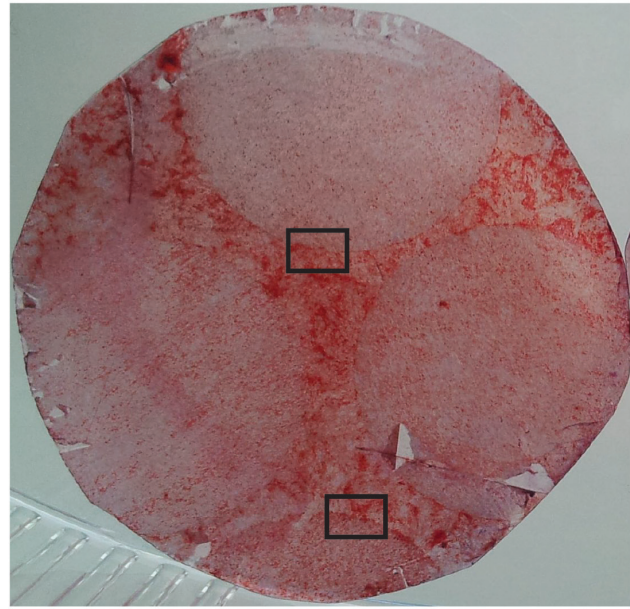

$1 \mathrm{~cm}$

e

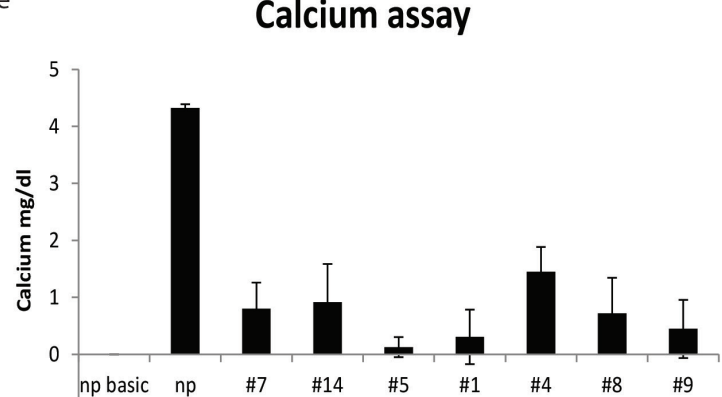

b

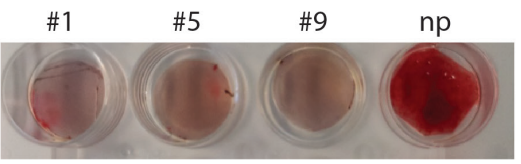

\#8

\#4

\#7

\#14

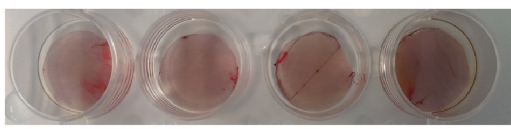

$+$

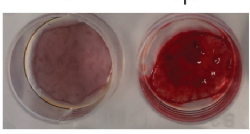

d
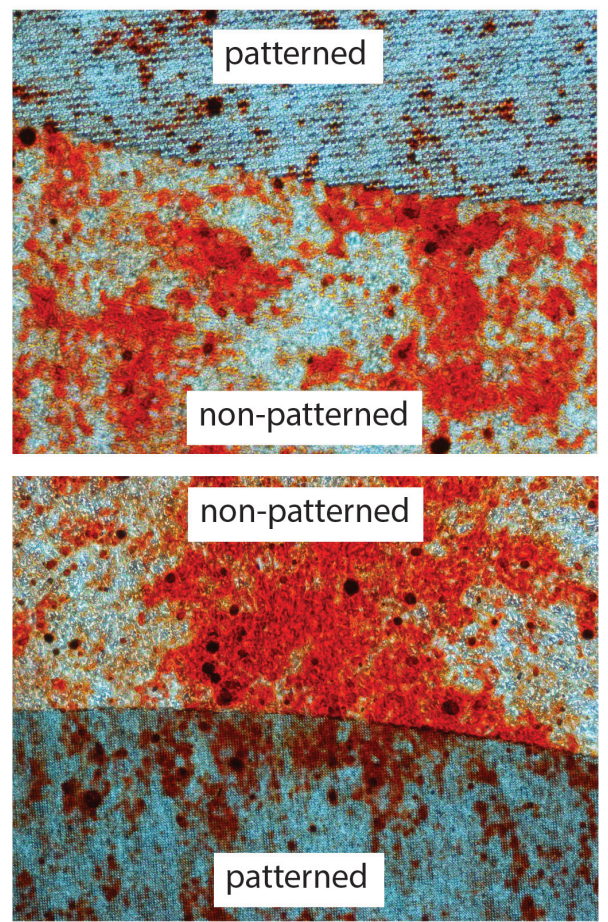

$100 \mu \mathrm{m}$

Figure 1: Polystyrene topographies inhibit the osteogenic response in hMSCs. a: Flow cytometry of hMSCS cultured for 5 days in basic medium on topographies. Bars show the average percentage of ALP positive cells. 10.000 cells were counted for each measurement. Positive control is osteogenic medium. b: Alizarin red staining of hMSCs cultured for 5 week in mineralization medium on topographies. Topographies are applied on $1.5 \mathrm{~cm}$ diameter discs, which fit in a 12 well-plate for cell culture. c: Alizarin red staining of hMSCS cultured for 5 week in mineralization medium on topographies in the same well. Larger $3 \mathrm{~cm}$ diameter discs, which contain several of the $1.5 \mathrm{~cm}$ diameter discs. The black rectangles indicate the area's which are shown in high magnification. d: Micrographs of area's on the large discs, showing the border between non-patterned surface and topographical surface. e: Calcium assay of hMSCS cultured for 5 week in mineralization medium on topographies. 
$\mathrm{np}$

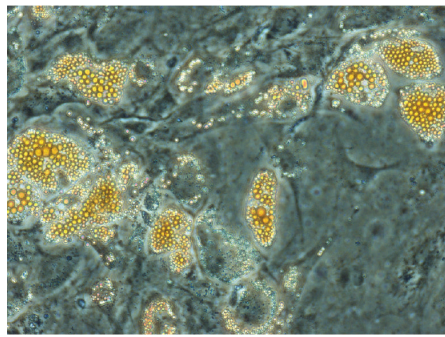

$\# 1$

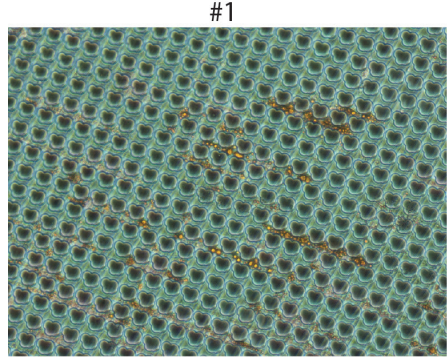

\#7

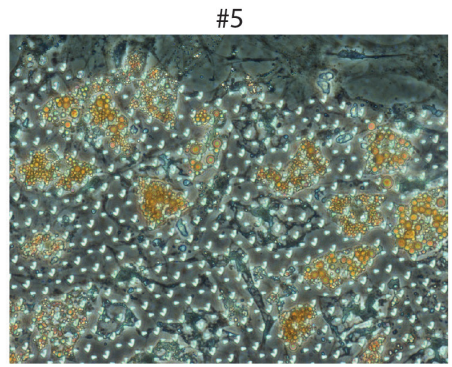

\#8

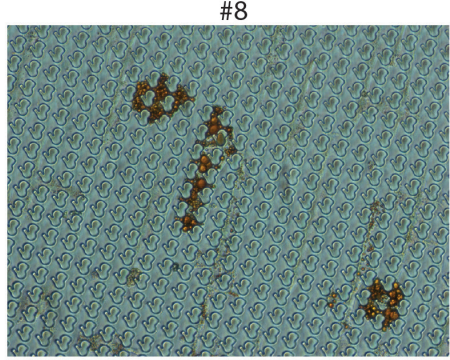

\#4

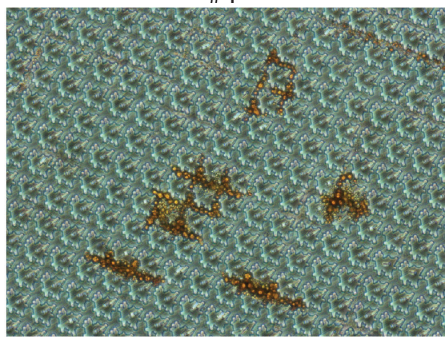

\#9

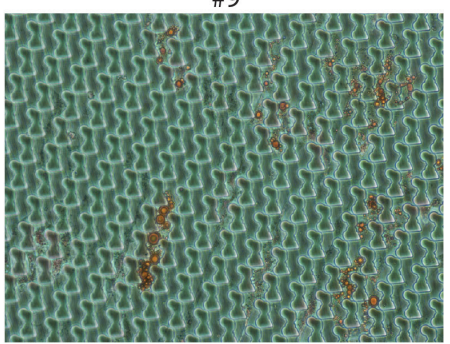

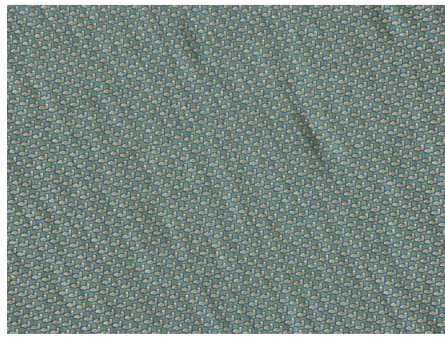

Figure 2: PS topographies inhibit adipogenesis, depending on feature size and spacing.

a: Representative bright field images of hMSCS on topographies after three weeks of culture in adipogenic medium.

After the discovery that PS topographies are able to inhibit mineralization of hMSCs we investigated if adipogenesis of hMSCS was also affected by the microtopographies. After 2 weeks of differentiation in adipogenic medium the lipid vesicles could be observed directly by microscopic images of oil red 0 staining (Fig. 2). The non-patterned polystyrene showed the highest degree of adipogenic differentiation with a high number of cells containing many lipid vesicles. All PS micro structures showed lower amount of cells with vesicles, strongly depending on the spacing of the features. Topographies \#4 and \#5, which have large spacing between topographies, show slightly less cells with lipid vesicles as the non-patterned surface. Topographies \#1, \#8 and \#9, which have moderate spacing between the topographies show about half as much cells with lipid vesicles, compared to the non-patterned surface. Topography \#7, which has very little spacing between the topographies, has no cells with lipid vesicles. It appears that the microtopographies inhibit both osteogenic and adipogenic differentiation of hMSCs. 
a

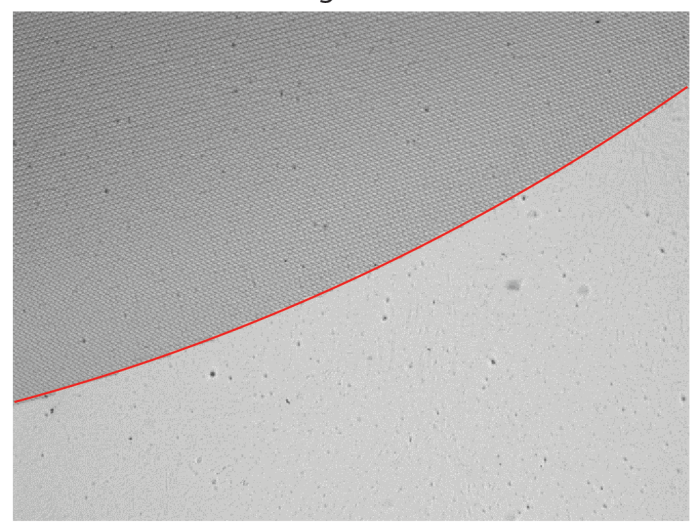

b

Ratios of nucleus quantification at the 2 day time point

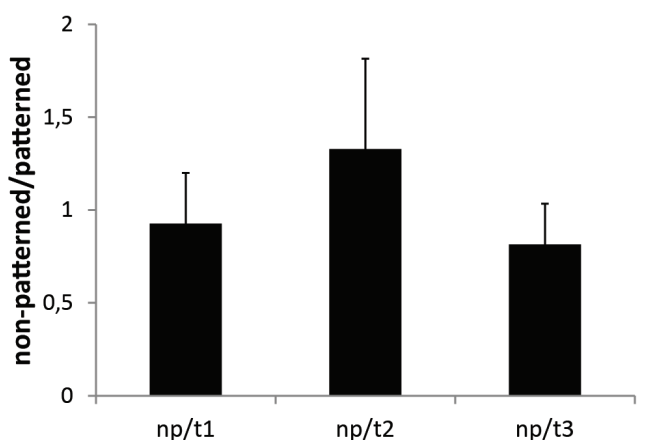

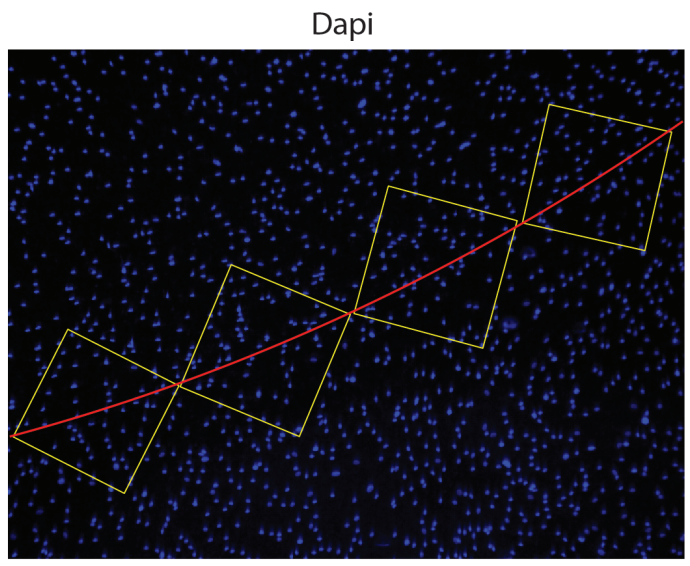

C Ratios of nucleus quantification at the 3 week time point

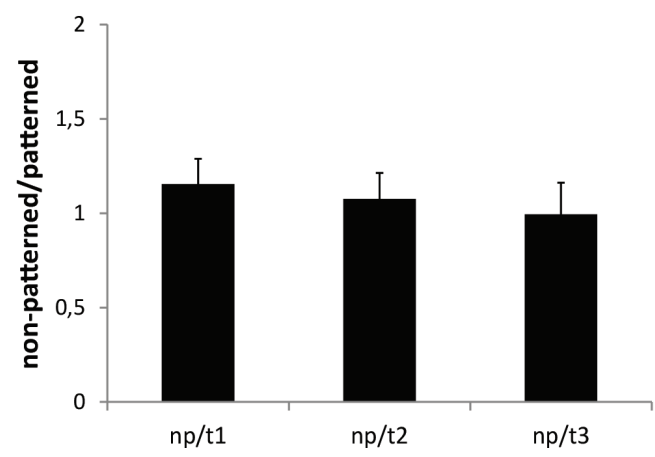

Figure 3: Nuclear quantification of hMSCs cultured on PS topographies a: Method to quantify nuclei. The bright field channel is used to determine the boundary between patterned and non-patterned areas. The boundary is used in the DAPI channel to count cells in adjacent areas with the same surface area to calculate ratios. b: Ratio of cells counted after 2 days of cell seeding. c: Ratio of cells counted after 3 weeks of mineralization.

Cell density has a big influence on hMSC differentiation (11). The finding that polystyrene microtopographies inhibit hMSc differentiation and mineralization could potentially be attributed to possible differences in cell-proliferation between hMSCs on topographies and non-patterned areas. To investigate differences in cell proliferation caused by microtopography, local cell numbers were counted on large PS films with micro-patterned areas. Local cell numbers were quantified by counting the nuclei in multiple adjacent regions along the boundary between non-patterned and patterned areas after staining with 4',6-diamidino-2-phenylindole (DAPI) (Fig. 3a). First the cells were quantified at a 2 day time point to observe cell attachment and early proliferation (Fig. 3b). Ratios between adjacent patterned and non-patterned areas were close to 1, which indicates minor differences in cell number. Secondly, the cell numbers at a 3 week mineralization time point were quantified as well (Fig. $3 \mathrm{c}$ ). There the ratios were also very close to 1 , with a trend for slightly (up to 10\%) more cells on the non-patterned area. These results suggest that there are small differences in cell number. However, we do not expect this small difference to be responsible for the large inhibitory effect of topography on hMSC mineralization. 
a

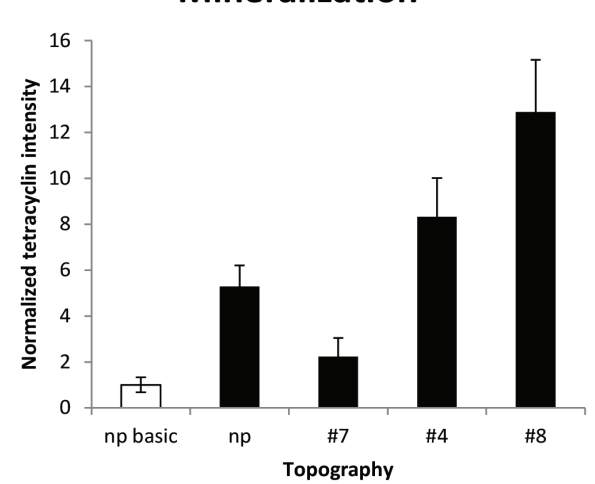

b
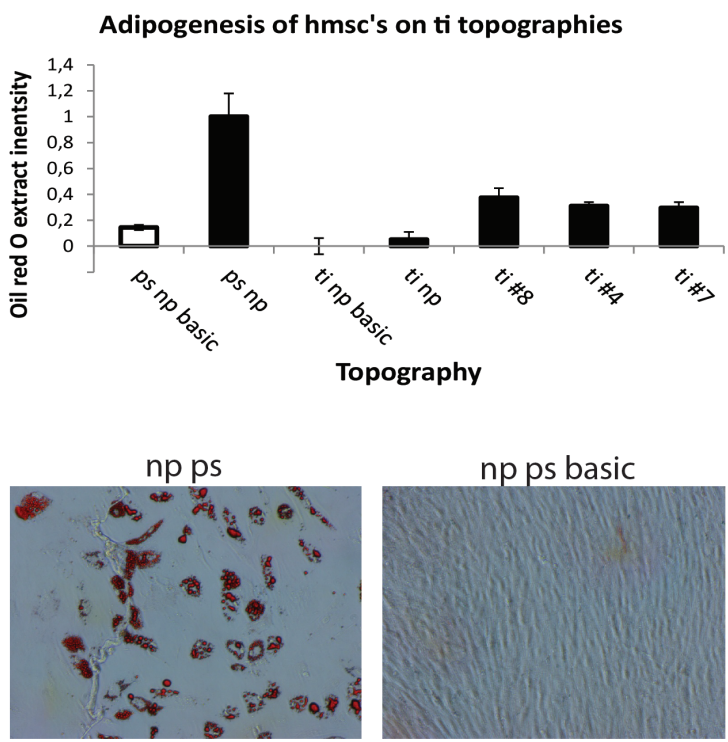

np ps basic

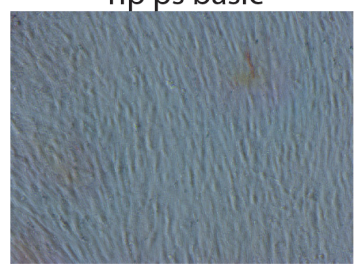

Figure 4: Differentiation of hMSCs on Ti topographies. a: Tetracyclin intensities of hMSCS cultured for 5 weeks on titanium coated PLA $1.5 \mathrm{~cm}$ diameter discs in mineralization medium. Averages are based on 5 images from 3 samples for each experimental group for a total of 15 measurements per group. b: Quantification of adipogenesis on Ti topographies. Respresentative images of the controls are shown for visual confirmation of lipid vesicles.

Originally the topography designs in this work were selected based on their ability to modulate the osteogenic differentiation of hMSCS when they were fabricated in Ti. The osteogenic effect of the Ti designed topographies was confirmed with a mineralization experiment (Fig. 4a). Non-patterned $\mathrm{Ti}$ and cell culture in basic medium (np basic) served as a negative control, while non-patterned Ti in osteogenic medium (np) served as a flat reference. In agreement with earlier studies (Chapter 3), the osteogenic positive hit topographies \#4 and \#8, increase mineralization of hMSCs, compared to non-patterned Ti. Topography \#7, which was not previously identified as osteogenic does not increase mineralization and even slightly decreases mineralization. In the case of osteogenesis the topographical cues are strongly dependent on the surface chemistry.

Next, to once again make a comparison between PS and Ti, hMSC adipogenesis was assessed on Ti topographies after 3 weeks of culture (Fig. 4b). Fat droplet formation could only be assessed indirectly on the Ti coated surfaces due to its opaqueness. Quantification of the eluted oil red 0 staining was normalized to the positive control, non-patterned polystyrene, which had pronounced formation of lipid vesicles. The negative control, non-patterned polystyrene in basic medium, which showed no lipid vesicles, still gave a background measurement of 0.14 . The non-patterned titanium did not show any oil red 0 staining, even less than the negative control. The titanium surfaces all showed very limited adipogenesis, of around 0.3. Taken together these results suggest that titanium coating strongly inhibits adipogenesis and that Ti coated topographies can minimally enhance adipogenesis but that it is still very minimal compared to polystyrene. 


\section{Discussion}

In this work, we demonstrated the instructive power of microscale topographies by showing for the first time total inhibition of mineralization by topography. Even at an early time point, the PS topographies reduce ALP expression in non-osteogenic medium, indicating inhibition of osteogenic differentiation. All topographies in our selection had a similar striking effect even though there is variation in size shape and spacing. One similarity that is shared by all the different feature designs is the height of $10 \mu \mathrm{m}$, which is roughly the diameter of an hMSC if it would occupy a spherical volume. Perhaps, this suggests that strong phenotypical changes can be achieved by topographies at dimensions that are similar to the cells themselves. There is not much literature with which we can directly compare our results due to the use of different materials, feature scales and cell types. One study which investigated the effect PS submicron grooves on rat MSC's showed that all grooved topographies slightly reduced ALP activity of up to $25 \%$, in line with our findings (12).

If one checks literature with other polymers, it is difficult to find any consistent trends concerning the effect of feature sizes and/or spacing optimal for enhancing osteogenesis. Experiments with submicron to several $\mu \mathrm{m}$ topographies made in polyurethane (PU) have shown that hMSCs are predisposed to differentiation towards the osteogenic lineage with small spacing of $1 \mu \mathrm{m}$ (7); submicron grooves fabricated in photo-curable polyurethane acrylate coated with gelatin showed very limited effect on mineralization (13); polymethylmethacrylate (PMMA) $120 \mathrm{~nm}, 100 \mathrm{~nm}$ deep pits with a controlled degree of spacing variance are able to induce osteogenic differentiation of hMSCs (2). No clear trends of topographical feature dimensions or spacing, promoting osteogenic differentiation of hMScs can be distinguished here.

In this study, PS microtopographies can inhibit lipid droplet formation in hMSCs depending on the spacing of the topographic features. If the cells have sufficient space between the features, they are able to differentiate and produce lipid vesicles. In the case of the topography with the minimal spacing, the cells do not fit between the features and are forced to be on top of the features. It seems that the mechanical effects on the cells there strongly inhibits adipogenesis. Other data suggests that PS submicron grooved topography slightly improve adipogenesis of rat MSC's (12). The difference in outcome there can most likely be explained by the difference in height of the PS grooves which was less than a micrometer, at least ten times smaller than the features in our study. When comparing to studies with other polymers our results are in agreement with studies with PU, where either flat or larger spacing of micro-pillars lead to more hMSC adipogenesis (7).

Cell counting experiments indicate that at an early time point are non-statistically significant differences between the patterned and non-patterned areas. These differences are most likely caused by local differences in cell density caused by cell-seeding. At a late time-point there is a trend of slightly more cells on the non-patterned areas, though it is unlikely that this small difference in cell-density has such a dramatic negative effect on the mineralization of hMSCs.

Previously published data and other studies have shown that Ti only slightly increases osteogenic differentiation of hMSCS (Chapter 3) $(14,15)$, most likely due to a difference in surface energy. Surface energy affects protein attachment to the surface which consequently affects the attachment of the cells (16). Our results clearly demonstrate a much stronger material specific effect when surface topography is involved: the same topographies which enhance osteogenesis on Ti substrates are able to inhibit mineralization on a polystyrene substrate. Furthermore, our data indicates that Ti strongly inhibits adipogenesis, regardless of microtopography. In this case the surface chemistry is highly domi- 
nant over the topography effect. The result is not completely surprising given by the fact that Ti promotes an osteogenic phenotype in mesenchymal cells.

On metals, literature suggests that osteogenic differentiation is enhanced by larger spacing of topographies on different feature scales. Tantalum coated silicon used for osteogenesis of Mc3t3 pre-osteoblasts (17) and dental pulp stem cells (18) showed that feature size and spacing and not shape were important. The effects were also a lot more significant with the pre-osteoblasts in comparison to the hMScs. Stainless steel microgrooves has been used to quantify fetal human osteoblasts mineralization (19). The scaling of topography was an order of magnitude larger and showed that spacing of 40 to $80 \mu \mathrm{m}$ improves mineralization.

Topography instructive effects are strongly dependent on the biomaterial surface chemistry. For these reasons It is vital to study topography on cells in the proper biomaterial context. Our study demonstrates for the first time the ability of microtopographies, fabricated in PS, to inhibit osteogenic and adipogenic differentiation of hMSCs. While topography size and spacing does not appear to be a determining factor to the degree of inhibition of osteogenesis, the spacing plays a clear role in the case of adipogenesis. Local cell counting measurements show that the inhibitory effect is most likely not caused by differences in cell density. When we compared these results to results obtained with the same cells and the same topography designs in Ti we come to the conclusion that the PS topographies influence hMSC differentiation in a different and in some cases opposite manner.

\section{Acknowledgements}

JdB, DS and FH gratefully acknowledge the financial support of the NanoNext NL initiative. JdB acknowledges the financial support of the Dutch province of Limburg. 


\section{Literature}

1. Tan KK, Tann JY, Sathe SR, Goh SH, Ma D, Goh EL, et al. Enhanced differentiation of neural progenitor cells into neurons of the mesencephalic dopaminergic subtype on topographical patterns. Biomaterials. 2015 Mar;43:32-43. PubMed PMID: 25591959. Epub 2015/01/17. eng.

2. Dalby MJ, Gadegaard N, Tare R, Andar A, Riehle MO, Herzyk P, et al. The control of human mesenchymal cell differentiation using nanoscale symmetry and disorder. Nature materials. 2007 Dec;6(12):997-1003. PubMed PMID: 17891143. Epub 2007/09/25. eng.

3. Olivares-Navarrete R, Hyzy SL, Park JH, Dunn GR, Haithcock DA, Wasilewski CE, et al. Mediation of osteogenic differentiation of human mesenchymal stem cells on titanium surfaces by a Wnt-integrin feedback loop. Biomaterials. 2011 Sep;32(27):6399-411. PubMed PMID: 21636130. Pubmed Central PMCID: PMC3350791. Epub 2011/06/04. eng.

4. Downing TL, Soto J, Morez C, Houssin T, Fritz A, Yuan F, et al. Biophysical regulation of epigenetic state and cell reprogramming. Nature materials. 2013 Dec;12(12):1154-62. PubMed PMID: 24141451. Epub 2013/10/22. eng.

5. Pittenger MF, Mackay AM, Beck SC, Jaiswal RK, Douglas R, Mosca JD, et al. Multilineage potential of adult human mesenchymal stem cells. Science. 1999 Apr 2;284(5411):143-7. PubMed PMID: 10102814. Epub 1999/04/02. eng.

6. Bianco P, Robey PG. Stem cells in tissue engineering. Nature. 2001 Nov 1;414(6859):118-21. PubMed PMID: 11689957. Epub 2001/11/02. eng.

7. Ahn EH, Kim Y, Kshitiz, An SS, Afzal J, Lee S, et al. Spatial control of adult stem cell fate using nanotopographic cues. Biomaterials. 2014 Mar;35(8):2401-10. PubMed PMID: ISI:000331679200001. English.

8. Li Y, Chu JS, Kurpinski K, Li X, Bautista DM, Yang L, et al. Biophysical regulation of histone acetylation in mesenchymal stem cells. Biophysical journal. 2011 Apr 20;100(8):1902-9. PubMed PMID: 21504726. Pubmed Central PMCID: PMC3077706. Epub 2011/04/21. eng.

9. Unadkat HV, Hulsman M, Cornelissen K, Papenburg B), Truckenmuller RK, Carpenter AE, et al. An algorithmbased topographical biomaterials library to instruct cell fate. Proceedings of the National Academy of Sciences of the United States of America. 2011 Oct 4;108(40):16565-70. PubMed PMID: 21949368. Pubmed Central PMCID: 3189082. Epub 2011/09/29. eng.

10. Hessle L, Johnson KA, Anderson HC, Narisawa S, Sali A, Goding JW, et al. Tissue-nonspecific alkaline phosphatase and plasma cell membrane glycoprotein-1 are central antagonistic regulators of bone mineralization. Proceedings of the National Academy of Sciences of the United States of America. 2002 Jul 9;99(14):9445-9. PubMed PMID: 12082181. Pubmed Central PMCID: PMC123160. Epub 2002/06/26. eng.

11. McBeath R, Pirone DM, Nelson CM, Bhadriraju K, Chen CS. Cell shape, cytoskeletal tension, and RhoA regulate stem cell lineage commitment. Developmental cell. 2004 Apr;6(4):483-95. PubMed PMID: 15068789. Epub 2004/04/08. eng.

12. Wang PY, Li WT, YU J, Tsai WB. Modulation of osteogenic, adipogenic and myogenic differentiation of mesenchymal stem cells by submicron grooved topography. J Mater Sci Mater Med. 2012;23(12):3015-28.

13. Kim J, Kim HN, Lim KT, Kim Y, Seonwoo H, Park SH, et al. Designing nanotopographical density of extracellular matrix for controlled morphology and function of human mesenchymal stem cells. Sci Rep. 2013;3:3552. PubMed PMID: 24352057. Epub 2013/12/20. eng.

14. Zhao G, Raines AL, Wieland M, Schwartz Z, Boyan BD. Requirement for both micron- and submicron scale structure for synergistic responses of osteoblasts to substrate surface energy and topography. Biomaterials. 2007 Jun;28(18):2821-9. PubMed PMID: 17368532. Pubmed Central PMCID: PMC2754822. Epub 2007/03/21. eng.

15. Olivares-Navarrete R, Hyzy SL, Hutton DL, Erdman CP, Wieland M, Boyan BD, et al. Direct and indirect effects of microstructured titanium substrates on the induction of mesenchymal stem cell differentiation towards the osteoblast lineage. Biomaterials. 2010 Apr;31(10):2728-35. PubMed PMID: 20053436. Pubmed Central PMCID: PMC2821717. Epub 2010/01/08. eng. 
16. Davies JE. Understanding peri-implant endosseous healing. Journal of dental education. 2003 Aug;67(8):932-49. PubMed PMID: 12959168. Epub 2003/09/10. eng.

17. Lovmand J, Justesen J, Foss M, Lauridsen RH, Lovmand M, Modin C, et al. The use of combinatorial topographical libraries for the screening of enhanced osteogenic expression and mineralization. Biomaterials. 2009 Apr;30 (11):2015-22. PubMed PMID: 19178942. Epub 2009/01/31. eng.

18. Kolind K, Kraft D, Boggild T, Duch M, Lovmand J, Pedersen FS, et al. Control of proliferation and osteogenic differentiation of human dental-pulp-derived stem cells by distinct surface structures. Acta biomaterialia. 2014;10(2):641-50

19. De Luca AC, Zink M, Weidt A, Mayr SG, Markaki AE. Effect of microgrooved surface topography on osteoblast maturation and protein adsorption. Journal of biomedical materials research Part A. 2015;28(10):35407. 


\section{NEW INSIGHTS INTO THE EFFECTS OF BIOMATERIAL CHEMISTRY AND TOPOGRAPHY ON MORPHOLOGY AND FUNCTION OF KIDNEY EPITHELIAL CELLS}

Frits Hulshof 1,2, Carolien Schophuizen ${ }^{3,4,5}$, Milos Mihajlovic ${ }^{5,6}$, Rosalinde Masereeuw ${ }^{5,6}$, Jan de Boer ${ }^{1}$, Dimitrios Stamatialis².

'Department of Cell Biology inspired Tissue Engineering, MERLN Institute for Technology-Inspired Regenerative Medicine, University of Maastricht, The Netherlands

Department of Biomaterials Science and Technology, MIRA Institute for Biomedical Technology

and Technical Medicine, University of Twente, Enschede, The Netherlands

${ }^{3}$ Department of Pediatric Nephrology, Radboudumc, Nijmegen, The Netherlands

4Department of Physiology, Radboudumc, Radboud Institute for Molecular Life Sciences,

Nijmegen, The Netherlands

${ }^{5}$ Department of Pharmacology and Toxicology, Radboudumc, Radboud Institute for Molecular Life Sciences,

Nijmegen, The Netherlands

${ }^{6}$ Div. Pharmacology, Utrecht Institute for Pharmaceutical Sciences, Utrecht, The Netherlands 


\section{Abstract.}

Increasing incidence of renal pathology in the western world calls for innovative research for the development of cell-based therapies such as a Bio-artificial kidney (BAK) device. To fulfill the multitude of kidney functions, the core component of the BAK is a "living membrane" consisting of a tight kidney cell monolayer with preserved functional organic ion transporters cultured on a polymeric membrane surface. This membrane, on one side, will be in contact with blood and therefore there should have excellent blood compatibility whereas on the other side should facilitate functional monolayer formation. In this work, we investigate in detail the effect of membrane chemistry and surface topography on kidney epithelial cells in order to improve functional monolayer formation. To achieve this, we fabricated designed microtopographies with high resolution and reproducibility on Polystyrene (PS) films and on Polyether Sulphone-Polyvinyl pyrrolidone (PES-PVP) porous membranes. A conditionally immortalized proximal tubule epithelial cell line (ciPTEC) was cultured on both and subsequently, the cell morphology and monolayer formation were assessed. Our results showed that L-dopamine (L-DOPA) coating of the PES-PVP was sufficient to support ciPTEC monolayer formation. The designed microtopographies of both materials affected ciPTEC morphology in different ways. Whereas the PS topographies with large features were able to align the cells in various patterns without significantly disrupting monolayer formation, the PES-PVP topographies with large features easily disrupted the monolayer. PES-PVP membranes with small features and with large spacing supported well the ciPTEC monolayer formation. In addition, the topographical PES-PVP membranes were compatible as a substrate membrane to measure organic cation transporter activity in Transwell ${ }^{\circledR}$ systems. Our study is the first one presenting new insights of the effects of surface topography on the adhesion, proliferation and morphology of kidney epithelial cells.

\section{Introduction}

Surface microtopography has a strong influence on the response of various cell-types to biomaterials (1-4). In fact, applying optimized surface topography to biomedical devices such as implants can improve tissue integration and host response. Optimal biomaterial-cell interaction is also extremely important for the development of bio-artificial organs where artificial membranes are combined with cells and tissues, such as in the bio-artificial kidney (BAK). A key requirement for such a device is the formation of a "living membrane" consisting of a tight kidney cell monolayer with preserved functional organic ion transporters, on suitable artificial membrane surfaces (5). The device should perform blood filtration and replace the endocrine, metabolic and immune-modulatory functions of the kidney $(6,7)$. Therefore, it may reduce the incidence of secondary morbidities in patients, and shorten dialysis duration or frequency, thereby improving the quality of life of patients with end stage renal disease (ESRD) $(8,9)$. In a BAK, one side of the artificial polymeric membrane is in contact with blood and therefore should be highly haemocompatible to prevent blood activation and coagulation, whereas the other membrane side should be cytocompatible and bioactive for adhesion of the renal epithelial cells. The living membrane should allow for transport of nutrients and solutes, including metabolic waste products to the cells for excretion, but also should block immune proteins from reaching the cells (Fig. 1). 


\section{Culture medium}

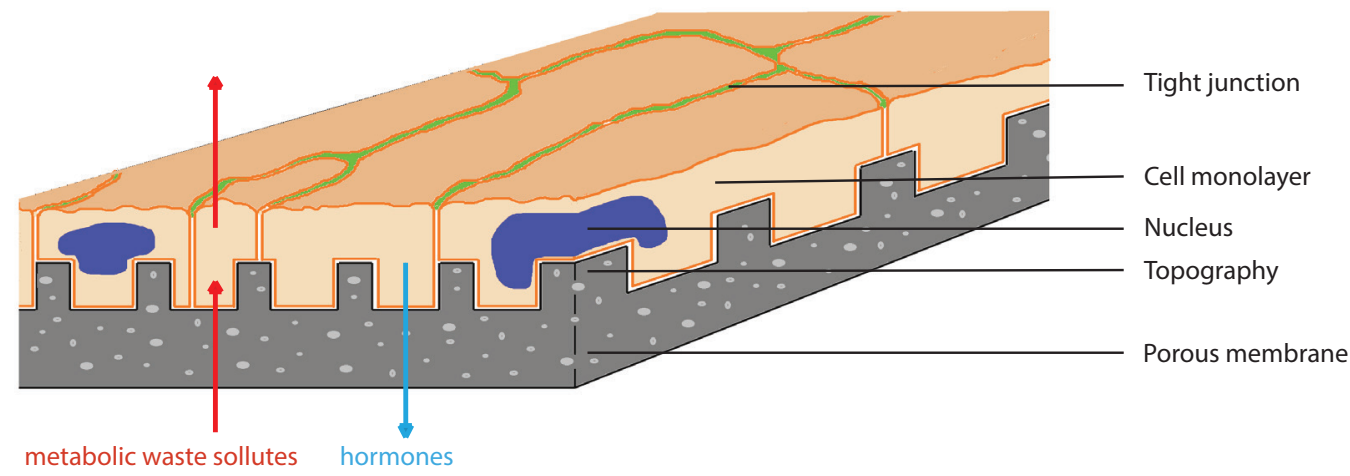

\section{Patient blood}

Figure 1: A 30 scheme of the "living membrane" concept to be applied to a bio-artificial kidney device. Kidney epithelial cells are cultured on a porous polymeric membrane, which separates the cells from components of the immune system in the blood of the patient, while allowing perfusion of blood toxins and cell metabolites. In this way the kidney cell layer is capable of removing small solutes and albumin bound compounds while replacing the endocrine, metabolic and immunemodulatory functions. This work investigates the effect of membrane surface topography to cell adhesion and alignment. Because monolayer quality is often assessed by staining of the tight junctions through fluorescently labelling ZO-1, this is schematically depicted in the figure.

Throughout the years, most studies on the development of a "living membrane" with renal epithelial cells have focused on improving cell adhesion on polyethersulfone (PES) and polysulfone based membranes by application of coatings of extracellular matrix components (10-12). However, in many cases maintenance of a trans-monolayer transport activity and monolayer integrity was not possible (13-17). Recently, we showed proof of concept of a living membrane by applying a double coating of 3,4-dihydroxy-L-phenylalanine (L-DOPA) and human Collagen IV (Coll IV) on PES membranes combined with conditionally immortalized proximal tubule epithelial cells (ciPTEC) (9). Very few studies have focused on the effect of membrane micro- and nanotopography on the kidney cell monolayer formation. Human embryonic kidney cell line (HEK-293) showed enhanced adhesion, proliferation on, and alignment to microstructured substrates (18). Submicron topography in combination with flow-induced shear stress (FSS) enhanced tight junction formation and cell alignment (19), leading to a more in vivo-like phenotype and faster tight junction formation (20). This suggests a correlation between kidney cell morphology and kidney cell function.

In this work, we investigate the effect of surface topography on renal epithelial cells. The ciPTECs were chosen as a cell model to investigate functional monolayer formation, because they possess several renal epithelial phenotypic features, including key transport proteins $(21,22)$. Previous high-throughput experiments performed in our lab using the Topochip screening platform $(4,23)$ identified a set of 7 microtopographies which are able to induce biological effects for various cells. For example, several of these topographies were able to induce strong cell orientation, even though the patterns were very different than those previously used for alignment of various cell types (24-26). Here, we produce these topographies on polystyrene (PS) films via hot embossing, to investigate ciPTEC response to topographies and on PES based porous membranes. The latter are selected due to their non-fouling properties and history as a material used in blood filtration systems, and compatibility with ciPTEC (9). The PES porous microstructured membranes were prepared by phase separation micro-moulding (PSHM), a method for combining surface 
topography and porosity in one step $(27,28)$. To the best of our knowledge, this is the first study that presents the development of a set of microstructured PS and porous PES substrates and investigates in detail the effects of surface topography on the adhesion, proliferation and morphology of kidney epithelial cells.

\section{Materials and Methods}

\section{Fabrication of Polystyrene microstructured films}

To investigate the effect of topography to kidney epithelial cells, 7 microtopographies were selected from an in silico library studied earlier (4). The topographies were produced on PS by hot embossing of a PS film (Goodfellow) as described previously (Appendix 1). Briefly, the inverse structure of the topographies was produced on silicon by standard photolithography and deep reactive etching. The silicon mould was used to make a positive mould on poly(dimethylsiloxane) (PDMS). The PDMS mould was required to create a second negative mould in OrmoStamp hybrid polymer (micro resist technology Gmbh), which serves as the mould for hot embossing the PS films. The fabrication of the Ormostamp ${ }^{\circledR}$ mould was necessary due to the fact that demoulding of PS from silicon often leads to destruction of the mould or the imprint. The hot embossing conditions were $140^{\circ} \mathrm{C}$ for 5 min at pressure of 10 Bar, with a demoulding temperature of $80^{\circ} \mathrm{C}$.

\section{Fabrication of PES based microstructured porous membranes}

Porous microstructured membranes were produced by PSHM. There, the appropriate solution is cast onto a microstructured master mold. Solvent and non-solvent liquid exchange initiates phase separation until the polymer solution contains sufficient non-solvent to precipitate. By selecting the right solvent/non-solvent system with the specific polymer, the porosity of the resulting membrane can be tuned. To increase mould hydrophilicity, a $100 \mathrm{~nm}$ silicon oxide layer was created on the mould by wet oxidation. The method was optimized using Topochip moulds fabricated by photolithography and directed reactive etching (4). The mould contains a pattern that creates a grid of walls with a height of $40 \mu \mathrm{m}$ and a width of $10 \mu \mathrm{m}$. between the walls are topographical structures with discrete shapes and a height of $10 \mu \mathrm{m}$. The walls are suitable structures to test fabrication of features with higher aspect ratios, which can be more challenging.

A Polyethersulphone (Fluka) / Polyvinylpyrrolidinone K90 (Fluka) (PES-PVP) solution was prepared in 1-methyl-2 pyrrolidinone (NMP) (Acros Organics). The solution was degassed and cast on the moulds. Two polymer dope compositions were tested: 10\% PES + 6.6\% PVP + 83.3\% NMP (W/W) and 15\% PES + $10 \%+75 \%$ NMP (W/W). Two non-solvent compositions were tested: demi water and 50\% demi water + $50 \%$ NMP (v/v). Two different phase separation temperatures were tested: room temperature and $1^{\circ} \mathrm{C}$. After casting, the mould with solution was quickly placed in the non-solvent bath. The solvent exchange was allowed to take place until complete demixing, which could last for up to 8 hours. Then, the porous microstructured membranes were washed in demi water. The demi water was refreshed three times a week for at least three weeks to remove all traces of NMP. In most cases the membranes were stored in $70 \%$ ethanol after washing. If the membranes were used immediately after washing, the membranes were sterilized with $70 \%$ ethanol for one hour prior to cell culture. 


\section{Cell culture and microcopy}

The ciPTEC, clone 21.2 were isolated from kidney tissue and cultured as described elsewhere (27). The cells were cultured in DMEM HAMS's F12 medium (Gibco) containing 10\% FCS (Lonza) $5 \mu \mathrm{g} / \mathrm{ml}$ Insulin, $5 \mathrm{\mu g} / \mathrm{ml}$ transferrin, $5 \mathrm{ng} / \mathrm{ml}$ selenium, $36 \mathrm{ng} / \mathrm{ml}$ hydrocortisone, $10 \mathrm{ng} / \mathrm{ml} \mathrm{EGF}$ and $40 \mathrm{pg} / \mathrm{ml}$ tri-iodothyronine. For the cell culture, the PES membranes were coated with either L-DOPA alone for 30 min or with combination of L-DOPA and collagen IV following the procedure described elsewhere (9). Briefly, 3,4-dihydroxyl-L-phenylalanine (L-DOPA) (D9628, Sigma Aldrich) was dissolved at $2 \mathrm{mg} / \mathrm{ml}$ in Tris buffer $10 \mathrm{mM}$ (pH 8.5) for 1 hour at $37^{\circ} \mathrm{C}$. Before culture, the coated membranes were washed with Hanks' balanced salt solution (HBSS; Gibco). The collagen IV coating solution of $50 \mu \mathrm{g} / \mathrm{ml}$ was prepared by dissolving collagen IV (Sigma Aldrich) for $2 \mathrm{~h}$ at $37^{\circ} \mathrm{C}, 5 \% \mathrm{v} / \mathrm{v} \mathrm{CO}_{2}$.

CiPTECS were seeded at a density of 60,000 cells $/ \mathrm{cm}^{2}$ on the membranes and before immuno-staining the cells were washed with HBSS and fixated for 5 min with 2\% paraformaldehyde, 4\% sucrose in HBSS. The cells were permeabilized with 0.3\% Triton X 100 (sigma Aldrich) in PBS for 10 min., before blocking with 2\% FBS, 2\% BSA, 0.1\% Tween in PBS. After blocking, the cells were incubated over-night at $4^{\circ} \mathrm{C}$ with primary antibodies (oxz-1 from Santa Cruz Biotechnology) in blocking solution. The membranes were washed 4 times 10 min. before secondary antibody (Goat anti rabbit-Alexa488 from Invitrogen) incubation of 1 hour at room temperature. After 4 more washing steps and 10 min. incubation in 4',6-diamidino-2-phenylindole (DAPI, from Sigma Aldrich) the membranes with cells were mounted with Mowiol for microscopy on a coverslip. Imaging was performed by a BD Pathway 435 automated fluorescence microscope. Image quantifications were performed by a custom made Cellprofiler pipeline (available on request) (28). The cell alignment was estimated by binning the data in $10^{\circ}$ wide groups and summing the 3 highest bin percentages to show the relative amount of cells aligned within the major $30^{\circ}$ angle.

\section{Scanning Electron Microscopy (SEM)}

The SEM images were taken by Philips XL-30 scanning electron microscope. Before scanning the samples were sputtered with gold for 40 seconds. From the SEM images the pore size was measured with Imagej (29).

\section{Transport measurements}

The trans-epithelial transport of $\left[{ }^{14} \mathrm{C}\right]$-creatinine and $[3 \mathrm{H}]$-inlulin by matured ciPTEC monolayers cultured on either polyester or PES-PVP membranes was measured using a Transwell ${ }^{\circledR}$ culture system. During the preparation of this experiment, membranes (stored in ethanol) were punched using a $12 \mathrm{~mm}$ skin biopsy puncher, and subsequently they were attached to the Transwell ${ }^{\circledR}$ culture system with custommade sealing rings. Then the L-DOPA coating was performed as described. All PES microstructured membranes and the reference Polyester Transwells ${ }^{\circledR}$ (diameter $12 \mathrm{~mm}$, pore size $0.4 \mu \mathrm{m}$ ) were coated with only L-DOPA solution, as described earlier. Mature cell monolayers were washed very carefully in modified Krebs-Henseleit buffer (Sigma-Aldrich, Zwijndrecht, the Netherlands) including $10 \mathrm{mM}$ Hepes (pH 7.4). Subsequently, all membranes were pre-incubated in Krebs-Henseleit - Hepes buffer $(0.5 \mathrm{~mL}$ apically, $1.5 \mathrm{~mL}$ basolaterally) for $2 \mathrm{~h}$ at $37^{\circ} \mathrm{C}, 5 \%(\mathrm{v} / \mathrm{v}) \mathrm{CO}_{2}$. Transport was initiated by the basolateral addition of either $\left[{ }^{14} \mathrm{C}\right]$-creatinine $(0.75 \mu \mathrm{M}, 2 \mu \mathrm{Ci} / \mathrm{ml})$ or $\left[{ }^{3} \mathrm{H}\right]$-inulin $(0.45 \mu \mathrm{M}, 20 \mu \mathrm{Ci} / \mathrm{ml})$ with or without cimetidine $(100 \mu \mathrm{M})$ and metformin (100 $\mu \mathrm{M}$; Sigma-Aldrich Co., Zwijndrecht, The Netherlands) as competitors for organic cation transport (35). At the start of the measurement, a $20 \mu \mathrm{L}$ reference sample was taken from the basolateral exposure compartment. After 30 min of incubation with gentle agitation 
at $37^{\circ} \mathrm{C}$, a $200 \mu \mathrm{L}$ sample was removed from the apical chamber. The activity of $\left[{ }^{3} \mathrm{H}\right]$ and $\left[{ }^{14} \mathrm{C}\right]$ in the samples was determined by liquid scintillation counting (Beckman). The fluxes of $\left[{ }^{14} \mathrm{C}\right]$-creatinine or $\left[{ }^{3} \mathrm{H}\right]$-inulin through the membranes were determined for each separate Transwell ${ }^{\circledR}$. The flux of $\left[{ }^{3} \mathrm{H}\right]$-inulin was used as an internal leakage marker.

\section{Results}

\section{Fabrication of Polystyrene non-porous microstructured films}

Figure 2 presents typical images of the topographies produced on PS by photolithography and hot embossing. Polystyrene was chosen for this study because it is the golden standard in tissue culture and it has beneficial optical properties for microscopy. This set provides a rather broad range of topographic features with round and sharp edges and size and spacing varying between 5 and $30 \mu \mathrm{m}$. The features on the topographies $\mathrm{t} 1$ and $\mathrm{t} 5$ are medium sized $(10-20 \mu \mathrm{m})$ with spacing of 5-10 $\mu \mathrm{m}$ whereas those on the $\mathrm{t} 2$ and t8 topographies are relatively large $(20-30 \mu \mathrm{m})$ with spacing of 5-10 $\mu \mathrm{m}$. The features on the topographies t6 and 7 are relatively small $(<5 \mu \mathrm{m})$ with large spacing of more than $20 \mu \mathrm{m}$ whereas on the topography t3 the features are small with almost no space between them.

t1

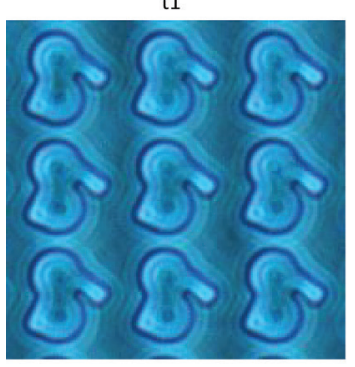

t5

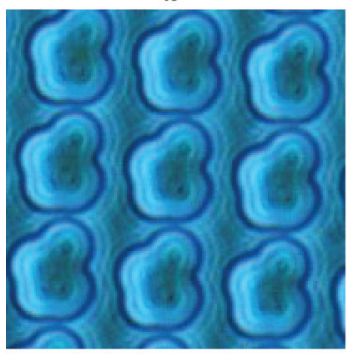

t8

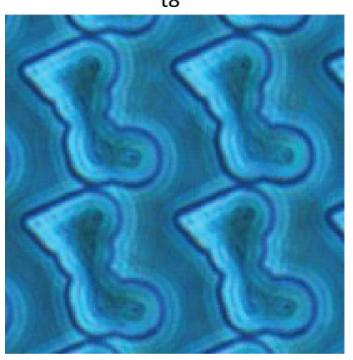

t2

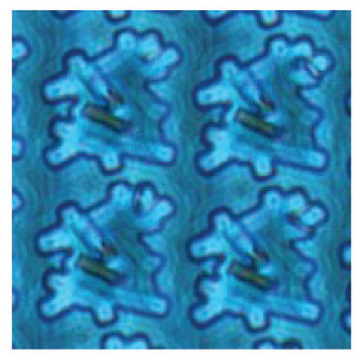

t6

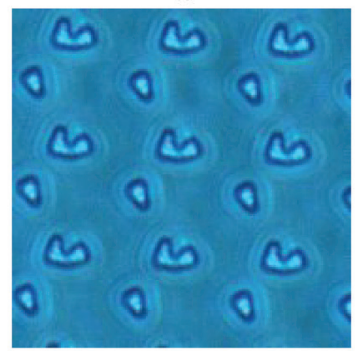

$50 \mu \mathrm{m}$

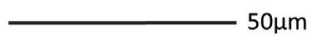

t3

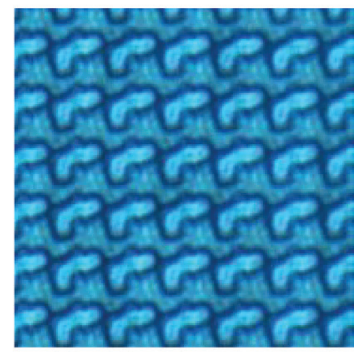

t7

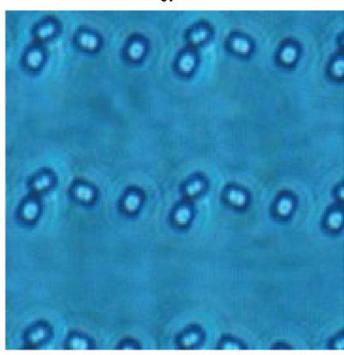

Figure 2: Optical microscopy images of polystyrene topographies produced by hot embossing. 
a

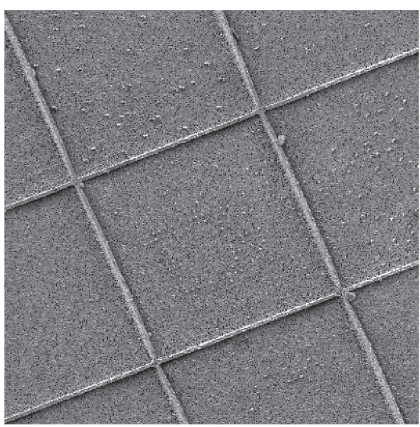

d

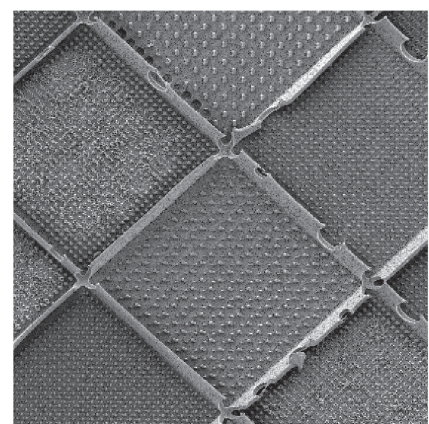

$300 \mu \mathrm{m}$

g

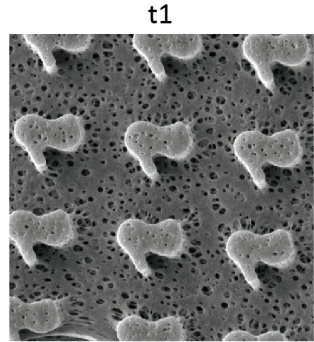

t6

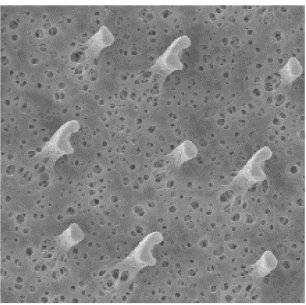

b

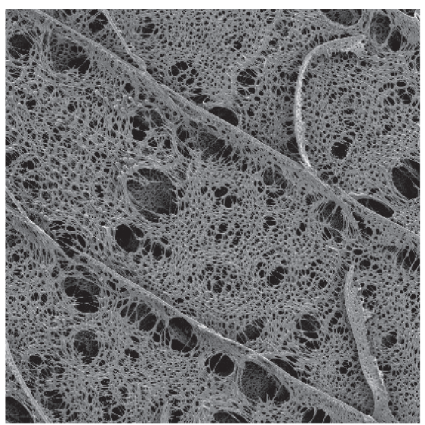

e

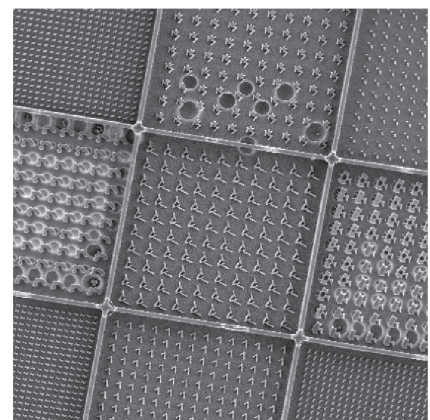

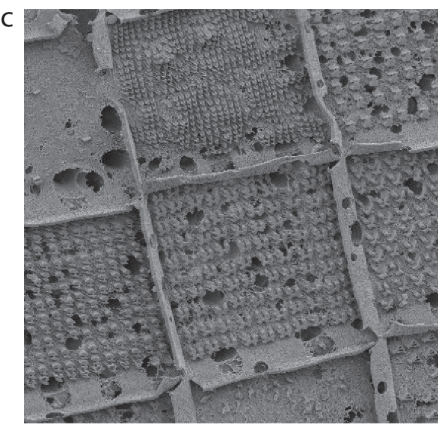

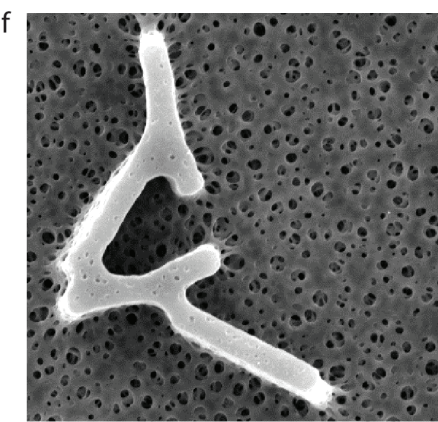

$10 \mu \mathrm{m}$

t3

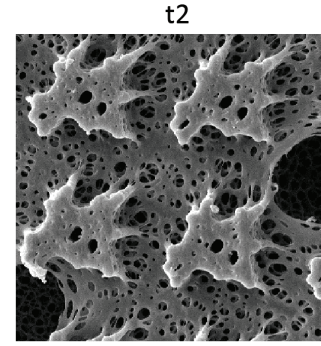

t7
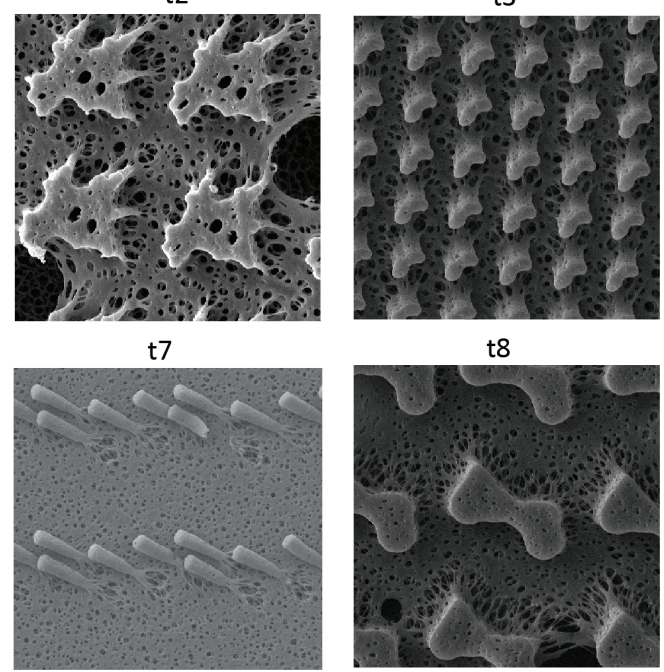

t8

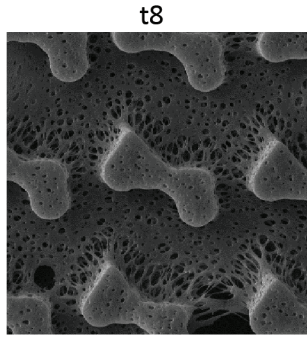

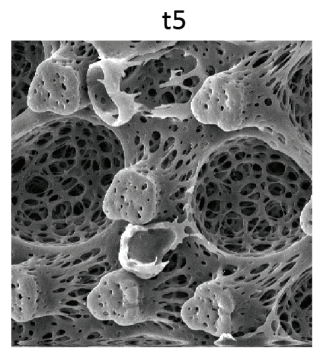

$-10 \mu \mathrm{m}$ h

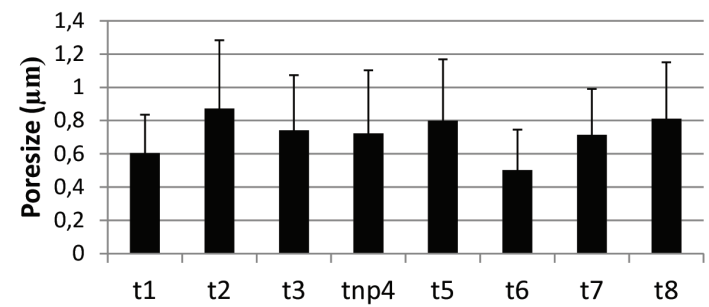




\begin{abstract}
Figure 3: Optimization of PSHM protocol for the fabrication of microstructured PES-PVP porous membranes.
a, b, c: SEM images of membranes with poor quality features due to poor interaction of the polymer dope with the hydrophobic silicon, due to low polymer concentration of 10\% PES/6.6\% PVP and due to phase separation with water as a non-solvent, respectively. d: SEM images of membranes with improved quality features due to phase separation with mixture of 50/50, NMP/demi water as non-solvent produced at $20^{\circ} \mathrm{C}$. e: SEM images of membranes produced via phase separation with $50 \mathrm{v} \% \mathrm{NMP} / 50 \mathrm{v} \%$ demi water at $1^{\circ} \mathrm{C}$. f: SEM image with higher magnification of the best quality topographies on porous membranes. g: SEM images of selected topographies produced on porous membranes. h: The mean pore size of topographical membranes. The error bars represent the standard deviation.
\end{abstract}

\title{
Fabrication of PES based porous microstructured membranes
}

Initial tests showed that the silicon moulds were very hydrophobic and the PES-PVP solutions could not be cast well resulting in films with poor feature replication (Fig. 3a). In fact, the polymer solution could not penetrate into the features and form the microtopographies and walls. To improve the interaction between the mould and the polymer solution, the moulds were coated with a $100 \mathrm{~nm}$ silicon oxide layer, created by wet oxidation. This allowed casting of the solutions successfully to the moulds. The casting of 10\% PES / 6.6\% PVP (w/w) in NMP solution with demi water as a non-solvent did not produce membranes with good quality features. The solution could penetrate the ridges, but the resulting walls were damaged during demoulding (Fig. 3b). The feature replication was also rather poor. Casting of a higher polymer dope concentration of 15\% PES + 10\% PVP in NMP (w/w) improved the quality of the replication of the topographies, but it was not yet satisfactory. In this case large voids were visible in the membranes and walls (Fig. 3c) probably due to the rapid demixing of solvent and non-solvent. To avoid this, we slowed down the demixing process, by using a mixture of 50\% demi water $+50 \%$ NMP (v/v) as a non-solvent. The new membranes have mostly intact walls with small defects and the topographical structures have better defined edges comparable to those produced by hot embossing (Fig. 3d). However, a significant amount of the features were deformed. The replication quality was improved by slowing down the demixing process even further by lowering the temperature of the coagulation bath, from room temperature $\left(20^{\circ} \mathrm{C}\right)$ to $1^{\circ} \mathrm{C}$. This new fabrication protocol of 15\% PES $+10 \%$ PVP (w/W) polymer solution in NMP with non-solvent consisting of a mixture $50 \% / 50 \%(\mathrm{~V} / \mathrm{v})$ demi water/NMP at $1^{\circ} \mathrm{C}$ produced porous microstructured membranes with features of excellent quality in the range of 5 and $30 \mu \mathrm{m}$ (Fig. 3e,f).

Figure $3 g$ shows representative SEM images of the 7 microstructured membranes used for the cell culture experiments (22). The pore size of the membranes was estimated via analysis of the SEM images. The membranes have large pores with a mean pore diameter of $0.8 \mu \mathrm{m}$ with rather large standard deviation of $0.34 \mu \mathrm{m}$ and there is no significant difference in pore size between the various microstructured membranes (Fig. 3h). This pore size is expected to allow free transport of water and uremic toxins through the membrane while it prevents the infiltration of the kidney epithelial cells, which have size of more than $20 \mu \mathrm{m}$ (22). The dimensions of topographies on PES membranes are quite similar to those on PS with a slightly lower resolution due to the porosity and shrinkage of $13.3 \%$ caused during the PSHM. For some of the larger topographies, for example $\mathrm{t} 2$ and $\mathrm{t5}$, there are occasionally crater shaped structures with a diameter between 10 and $20 \mu \mathrm{m}$ and a depth of 5-10 $\mu \mathrm{m}$ close to the topographies (Fig. 3g). These small artefacts are not frequent and they should not have a significant effect on the topography response. The crater like structures have the similar porosity to the rest of the membrane surface and are not expected to affect the transport properties of the membranes. 


\section{Cell culture on PS films}

Surface topography affects morphology and function of many cell types (31). In the case of kidney epithelial cells, other studies have indicated that topography can induce alignment resulting in a more in vivolike phenotype and faster tight junction formation (19). Here, the CiPTECs were cultured for one week on the 7 microstuctured PS films and a non-patterned polystyrene film, as a reference. The morphology of the cells was visualized by staining filamentous actin with phalloidin, tight junctions with Zona Occludens 1 (Z01) and the DNA with Dapi. Z01 is a critical component of tight junctions which enable cells to form an impermeable barrier to solutes $(32,33)$. Because of this, it acts as a marker for cell polarity and integrity of the cell monolayer.

The ciPTEC form confluent cell layers on all PS films (Fig. 4a). The PS substrates support the adhesion and proliferation of ciPTECs well, without the need of applying any coatings. Besides, the surface topography strongly influences cell morphology. For the topographies with relatively large features (t1, t2, t5 and t8) the cells attach between the microstructures, become rather thin and elongated and form a grid-like pattern which aligns them. For the topographies with smaller features (t3, t6 and t7) the cells align without creating grid-like patterns and do not alter the cell morphology much compared to the non-patterned surface, tnp4. The cells are guided by the smaller topographies but are not confined by them.

The cell alignment in the confluent monolayers was quantified by measuring the nuclear alignment with Cell Profiler software (29) (Fig. 4b). Two of the topographies (t3 and t5) show high degrees of alignment. Representative images of nuclei and corresponding alignment distributions are shown in Figure $4 \mathrm{c}$ and $4 d$, respectively. Clearly visible are the high alignment of t3, low alignment of $t 6$ and alignment on the non-patterned surface tnp 4 . The t3 has the highest alignment of 54\% of cells within $10^{\circ}$ of the main axis. This is higher than the previously reported alignment of ciPTEC on grated topographies, which was 35\% (20). This demonstrates that properly designed microscale structures can achieve alignments that are the same or better than grated nano-topographies. It is important to note that the cells on the non-patterned substrate (tnp4) also show considerable alignment due to the fact that cells in confluent layers tend to align in bundles on a 2-dimensional surface. However this alignment is not always in the same direction. In fact the overall alignment of cells over a larger surface of more than a $1 \mathrm{~cm}^{2}$, is around $31 \%$ within $10^{\circ}$ of the major axis. Finally other topographies such as t6 induce much lower cell organization and alignment suggesting that perhaps the features there disturb the formation of confluent cell monolayer.
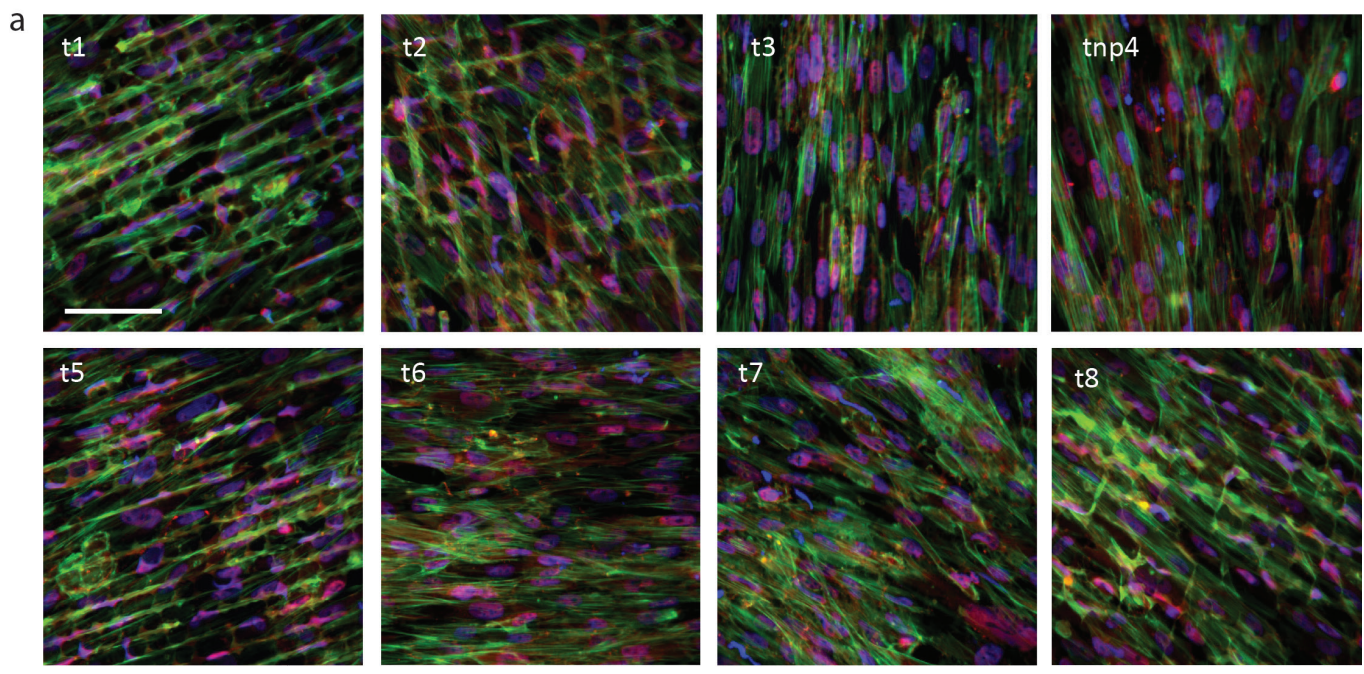
b

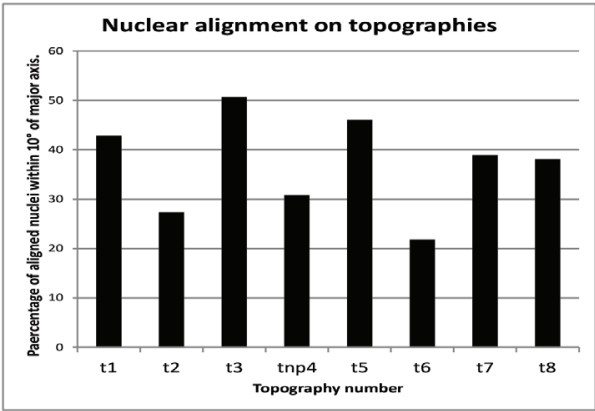

C

t3

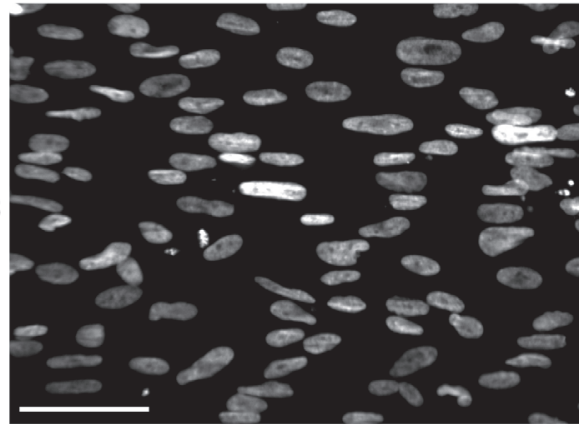

t6

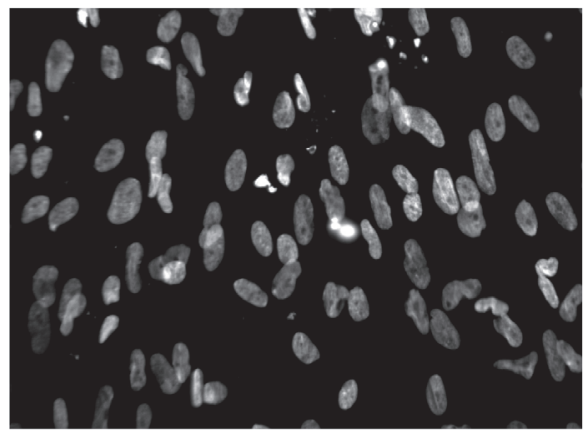

tnp4

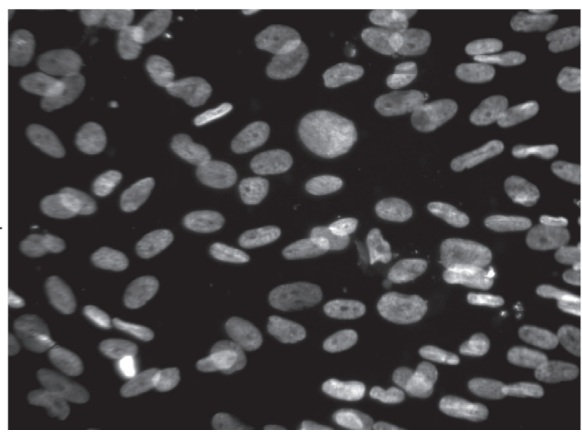

d
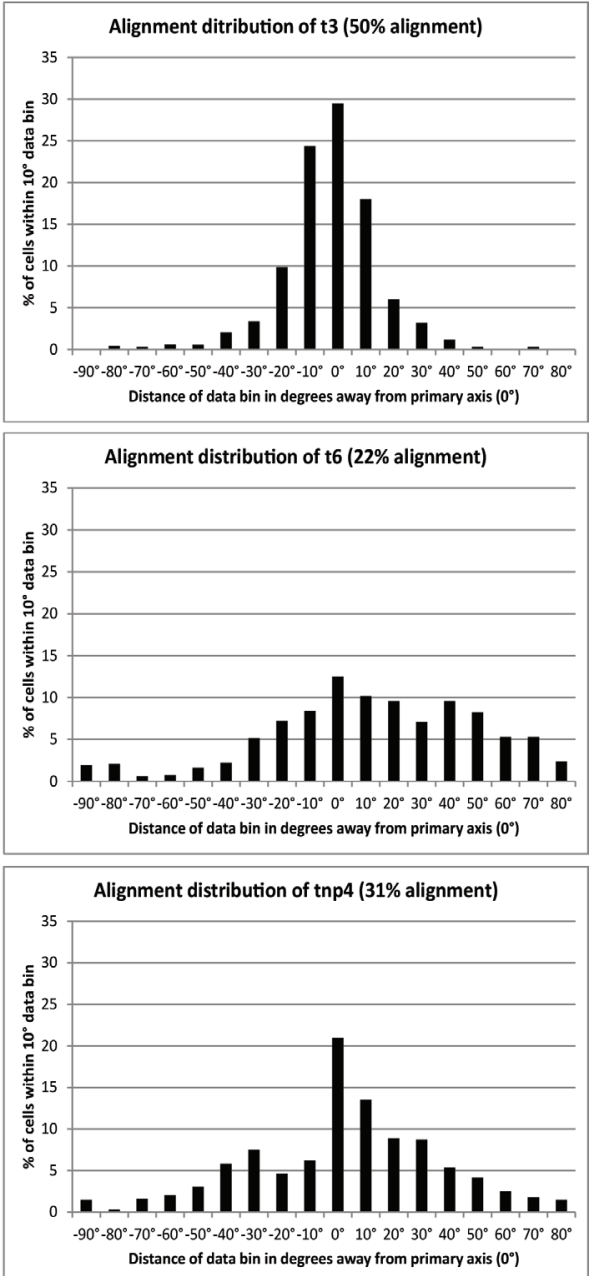

Figure 4: Morphology and alignment analysis of ciPTEC on polystyrene topographies.

a: Fluorescence microscopy images of ciPTEC cultured on PS topographies, stained for DNA (blue), Filamentous actin (green), 20-1 (red). Scale bars are $100 \mu \mathrm{m}$.

b: Quantification of alignment of nuclei of ciPTEC within $10^{\circ}$ of the major axis on topographies. c: representative fluorescence images of nuclei which were used for quantification. d: distributions of alignment corresponding to nuclei of (b). 


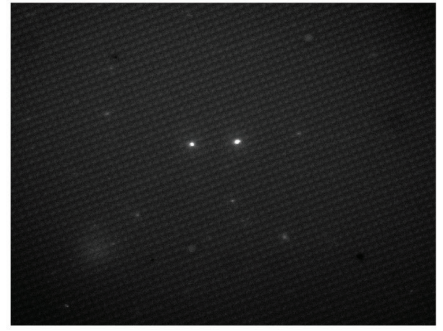

$600 \mu \mathrm{m}$ b

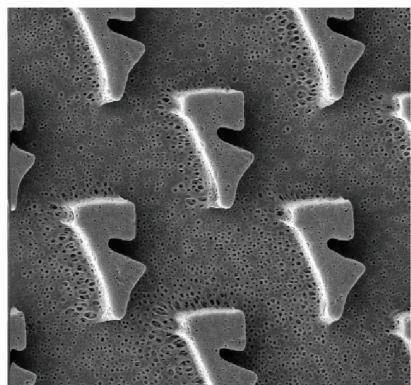

- $10 \mu \mathrm{m}$

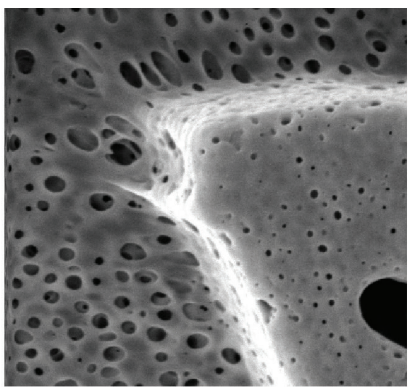

$5 \mu \mathrm{m}$

C
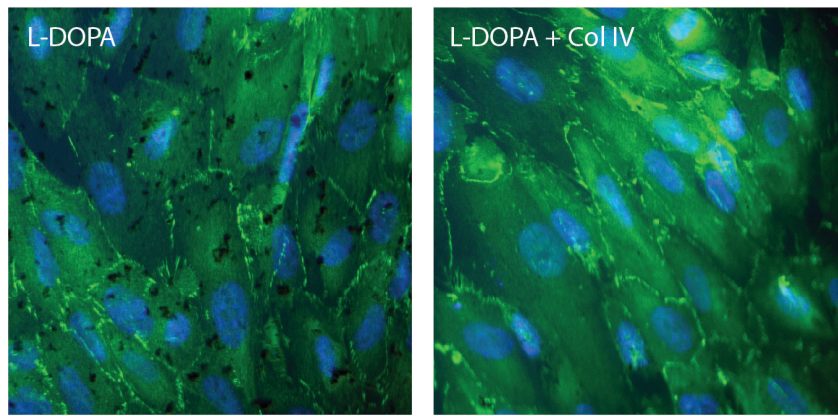

$100 \mu \mathrm{m}$

d
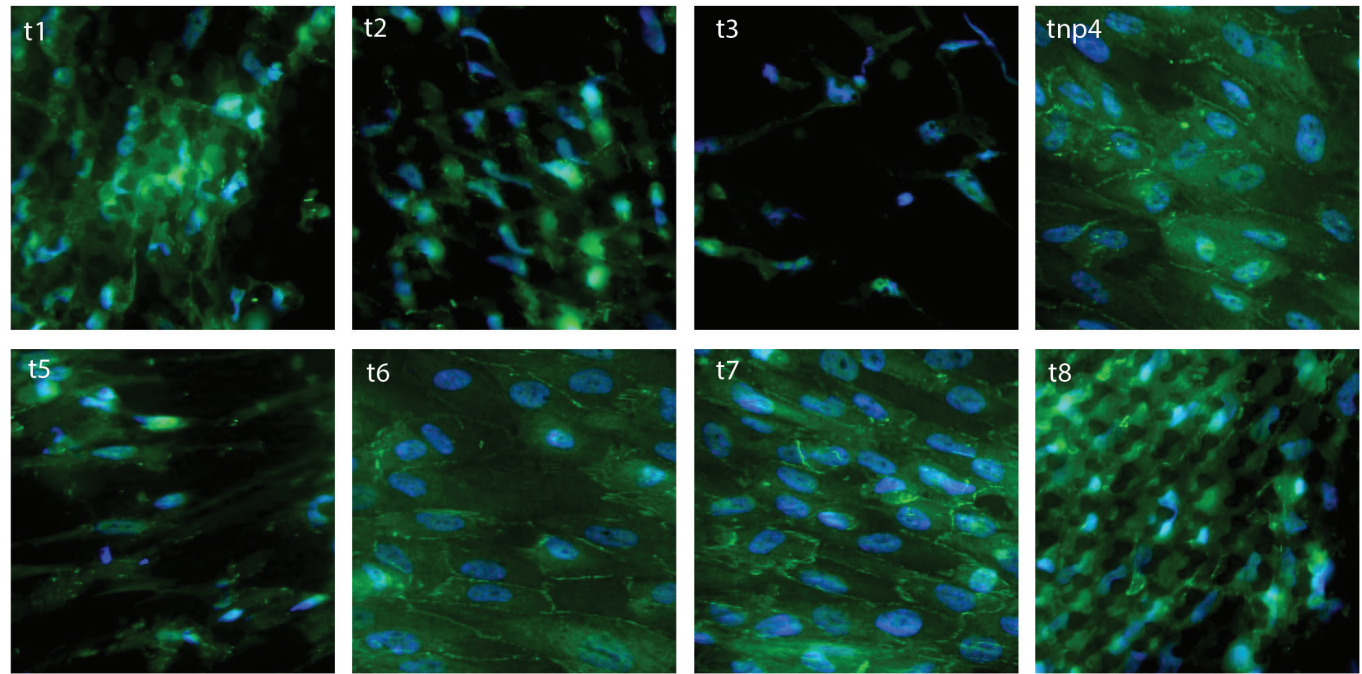

$100 \mu \mathrm{m}$

Figure 5: Conditionally immortalized kidney epithelial cells (ciPIEC) cultured on PES-PVP micro structured porous

membranes. a: Fluorescence microscopy image of ciPTEC cultured on uncoated PES-PVP membranes stained, with Dapi. b: SEM image of a topographical feature on a porous PES membrane. c: Fluorescence microscopy image of ciPTEC cultured on L-DOPA coated PES membranes and double coated membranes (L-DOPA + Collagen IV) PES membranes, stained with Dapi (blue) and ZO-1 (green) d: Representative images of ciPTEC cultured on L-DOPA coated porous PES micro structured membranes. 


\section{Cell culture on PES-PVP membranes}

To assess response of ciPTECS to the PES-PVP microstructured membranes the cells were cultured there for one week. Due to the non-fouling properties of PES the cell adhesion was extremely poor as only several cells were visible on an area as large as $1 \mathrm{~cm}^{2}$ (Fig. 5a). To address this issue, the membranes were coated with either L-DOPA or with a double coating of L-DOPA and Collagen IV. Earlier work showed that a double coating of L-DOPA and Collagen IV provided the best substrate for ciPTEC monolayer formation (9). The L-D0PA coating is expected to create a negatively charged surface, to increase membrane hydrophilicity $(9,14$, 34) and to increase adhesion of ECM proteins to the surface, thereby increasing cell adhesion. The coating(s) were applied to one side of the membrane only, leaving the other side of the membrane resistant to fouling. In this work, we optimized the coating procedure in order to achieve coating layers in the nanometer range and avoid changing the quality of the microstructured features (Fig. 5b). When we assessed the effect of the coatings on ciPTECs we observed that both the morphology and quality of cell monolayers on PES membranes coated only with L-DOPA or double coating L-DOPA / Coll IV are identical (Fig. 5C). This is probably due to the use of the ciPTEC clone 21.2 which were isolated from kidney tissue and can produce rather quickly their own ECM (22). In contrast to ciPTEcs isolated from urine (21) and other primary kidney cells (14) which require coating with Col IV, the 21.2 ciPTEC cells do not require this. Although more detailed investigation is required, this finding suggests that 21.2 cells are excellent candidates for application in a BAK without the need of expensive and time-consuming coatings of Coll IV.

When comparing ciPTEC morphology cultured on non-patterned PS and L-Dopa coated PES membranes, the cell monolayers look very similar and homogenous. On both materials the cells seem to form tight monolayers with clearly formed protein junctions between the cells, as concluded from the Z0-1 staining. When comparing the effect of the topography on the cells for both materials, the microstructured PES-PVP membranes seem to have a more dramatic effect on the ciPTEC morphology and monolayer integrity in comparison to PS. In fact, the large features $(t 1, t 2, t 5, t 8)$ have a more disruptive effect on the cell monolayer, big holes can be seen on the cell monolayer (Fig. 5d), whereas for the topographies $\mathrm{t} 1$, t2 and t8, the cells form small islands with poorly defined tight-junctions. The topography $t 3$, which has medium sized features with small spacing between them, has a low number of cells. However, for the topographies with smaller features, such as $t 6$ and $t 7$, a good cell monolayer is created, and in the case of $t 7$, the features also seem to provide cues for cell alignment.

\section{Creatinine transport through the microstructured PES-PVP membranes}

Earlier studies have shown that ciPTEC can achieve active transport of organic cations from the basolateral side to the apical side $(9,35)$. Here, we compare the transport properties of the microstructured membranes with topographies $\mathbf{3}$, $t 6$ and 77 to the non-patterned membranes (tnp4) and polyester (PE) control membrane (Transwell ${ }^{\circledR}$ ). The membranes with topographies t6 and t7 were selected since our earlier study showed that they seem to support formation of a ciPTEC monolayer, whereas the membrane with topography t3 was chosen as a negative control since it disrupts the monolayer. To determine active transport by the cells, the creatinine flux was also measured in the presence of cimetidine, which competes with creatinine as a substrate for the organic cation transport proteins. To estimate monolayer leakage, inulin flux was also measured for every membrane because it crosses the epithelial membranes only by diffusion (36). Supplementary Figure $\mathbf{S 1}$ shows the results of two experiments. The high variability between experiments greatly reduced the significance of the data. Still, clear trends can be seen. The non patterned (tnp4), the microstructured PES-PVP membranes with topography t7 and the commercial polyester membranes (PE nP), all coated with L-DOPA alone, can actively transport creatinine, which decreases with addition of cimetidine (see all membranes + cim). Based on these preliminary data, there 
is no improved active creatinine transport observed in ciPTEC cultured on micro patterned membrane.

\section{Discussion}

In this work, we investigated in detail the effects of the biomaterial surface topography on the adhesion, proliferation and morphology of kidney epithelial cells. For this, a set of microstructured PS (as a golden standard for cell culture studies) and PES based membranes (state of art material for (bio)artificial kidney devices) were developed and studied. A significant effort was put on the fabrication of excellent quality porous microstructured PES membranes. Important parameters to be tailored were the polymer concentration, temperature and the composition of the coagulation bath. In fact, a high polymer concentration, a decrease of the coagulation bath temperature from $20^{\circ} \mathrm{C}$ to $1^{\circ} \mathrm{C}$ and the use of non-solvent mixture of $50 \% / 50 \%(\mathrm{~V} / \mathrm{V}$ ) demi water/ NMP resulted in the production of porous (mean pore size of $0.8 \mu \mathrm{m}$ ) microstructured membranes with features of excellent quality.

The ciPTECS were successfully cultured on both PS and the PES-PVP membranes. An important finding of this work is that a good quality cell monolayer can be achieved on porous PES membranes with the application of a coating with L-DOPA, without the need of extra coating with collagen IV, as found in earlier stu-dies with ciPTECs isolated from human urine (9). This is likely because the ciPTEC clone used in this study, which was originally derived from kidney issue, shows higher expression of extracellular matrix genes collagen I and -IV as compared to ciPTEC that originated from urine (22). This may be a compelling explanation of why the ciPTEC in these experiments did not require additional Collagen IV coating.

Another important finding of this work is the striking difference between the response of ciPTECs on PS and PES membranes. While for PS the large topographic features did not adversely affect ciPTEC cell numbers and monolayer formation, the same features fabricated on PES disrupted the cell monolayer. For the PES membranes the topographies with small features did not disrupt the monolayer and were able to induce cell organization and alignment. Alignment of kidney epithelial cells makes them more susceptible to mechanical cues such as flow-induced shear stress, which improves tight junction formation $(19,20)$.

The literature concerning the effect of substrate topography on kidney cells is quite limited. Polystyrene ridged nanostructures with depths of $100 \mathrm{~nm}$ and periodicity of $430 \mathrm{~nm}$, created by laser irradiation were able to increase the alignment of human embryonic kidney cells (HEK-293) (18). Compared to our designed microstructures, the ridges in Rebolar et. al. (18) are more than 2 orders of magnitude smaller, yet the degree of alignment is very similar to ours. An interesting question for future work is whether the cell alignment is caused by the same mechanism at two different feature scales. In another study, PS ridges with a width and depth of 0.75 microns were able to support HK2 cells and induce alignment of up to $24 \%$ in static flow conditions (19). Some of our PS topographies are at least one order of magnitude larger and are able to induce alignment of up to $54 \%$. As mentioned earlier alignment of kidney epithelial cells can improve tight-junction formation. Ridge topographies with very similar dimensions were also applied to polycarbonate membranes with large pores between 3 to $12 \mu \mathrm{m}$ (20). There, the features were very small in comparison to the membrane pores, opposite to our case, where our membranes have large topographical features in comparison to the pores. This is possible because here the topography and porosity is formed in one step, instead of being created via subsequent embossing. This is actually an important advantage of PSHM in comparison to hot embosing post membrane fabrication, which can significantly alter the porosity, especiallly in the case of a membrane with relatively small pores. Despite these significant effects of membrane topography to cell morphology, preliminary transport experiments through selected microstructured PES membranes with good quality cell monolayer and cell orientation did not show improved active creatinine transport, in comparison to the non-patterned membranes. 
a

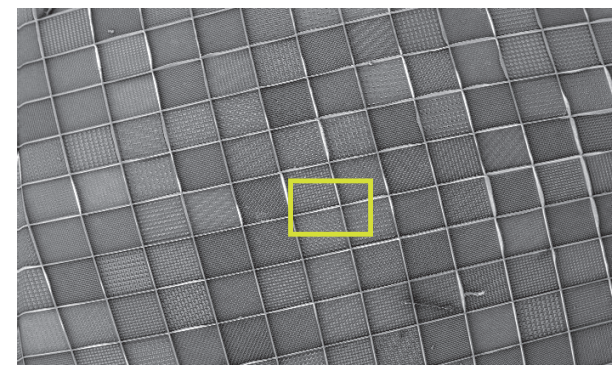

$500 \mu \mathrm{m}$
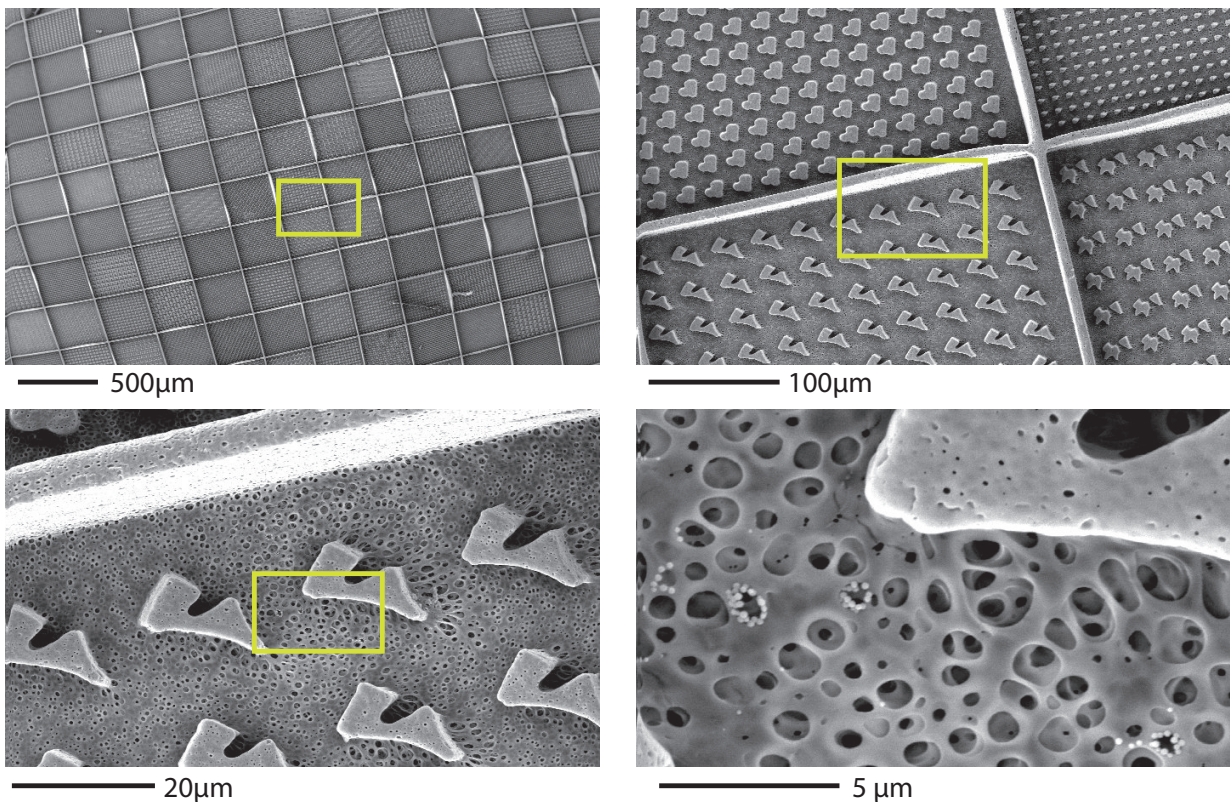

b
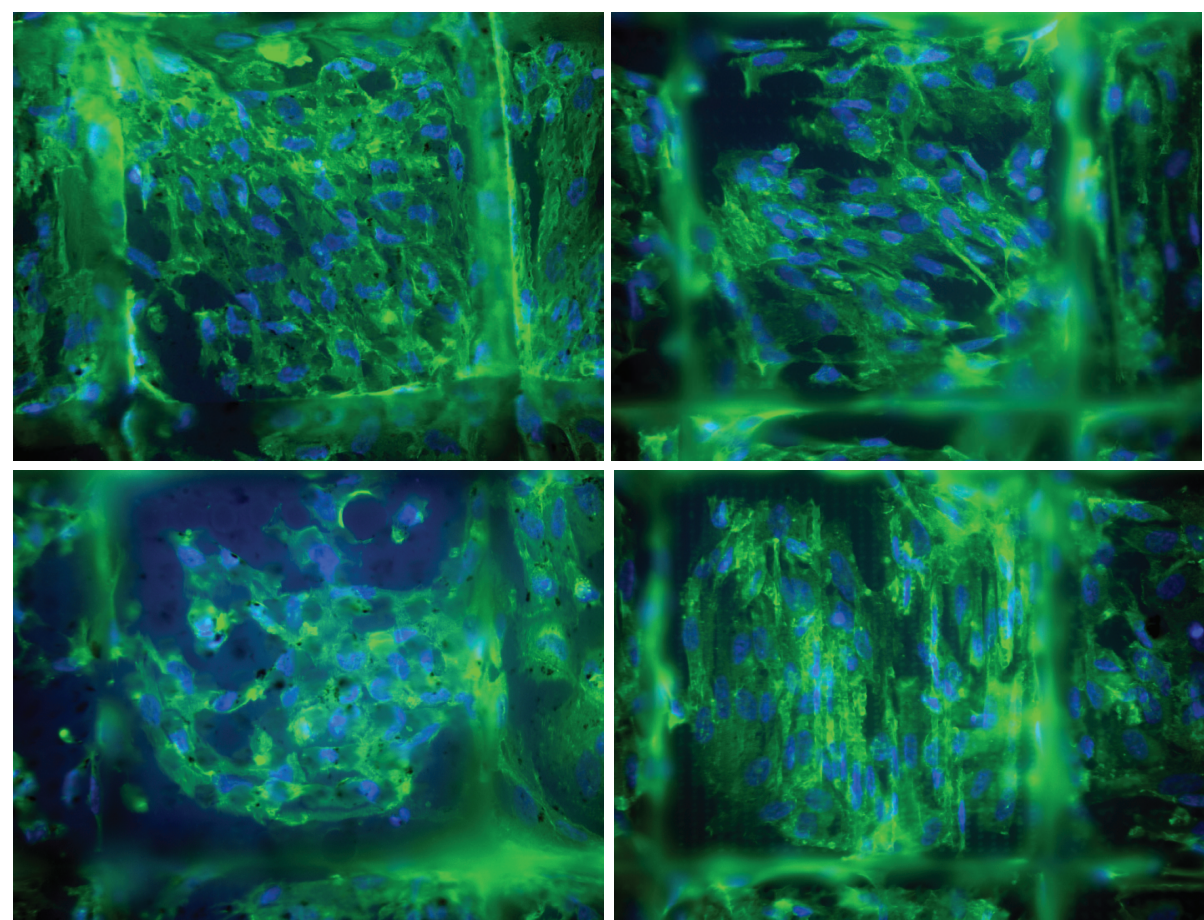

$100 \mu \mathrm{m}$

Figure 6: Porous PES TopoChips and culture of Kidney epithelial cells (ciPTECS).

a: SEM images of a porous PES TOPOChips. Yellow brackets indicate the location of the following zoomed in image. b: Fluorescence microscopy image of ciPTEC cultured on porous PES Topochips, stained with Dapi (blue) and oxZ0-1 (green). 


\section{Conclusion and outlook}

In this work, we investigated the effect of surface topography of PS and PES-PVP membranes on the morphology, adherence and function of kidney epithelial cells. For both materials the microtopography affects functional monolayer formation, morphology and alignment of ciPTECS and cell monolayer can be formed without the need of ECM coatings. In contrast to the non-porous PS topographies, for most of the porous PES-PVP membranes with large features the cell monolayer is disrupted. The PES-PVP membranes with small features and with large spacing between the features support the ciPTEC monolayer formation well. Here, only a limited number of topographies were studied while the Topochip high-throughput screening platform (4), from which this set was derived, has more than 2100 different topographies. Following the protocol developed here, we have also performed preliminary experiments of fabricating complete porous PES TopoChips (Fig. 6a). The ciPTEC culture on these porous Topochips (using only L-DOPA coating) also showed significant effect of the topography on the cells (Fig. 6b). Our future work will focus on developing a sensitive image based assay to screen the high number of topographies developed and to identify topographies beneficial for the development of a 'living membrane' for the BAK device.

\section{Acknowledgments}

JdB, DS and FH gratefully acknowledge the financial support of the NanoNext NL initiative. JdB acknowledges the financial support of the Dutch province of Limburg, This research was supported by BioMedical Materials research program (P3.01 BioKid), co-funded by the Dutch Ministry of Economic Affairs. Also, the financial contribution of the Dutch Kidney Foundation is gratefully acknowledged and the Marie Curie ITN project: BIOART (grant no.316690, EU-FP7-PEOPLE-ITN-2012).

\section{Supplementary figure}

a

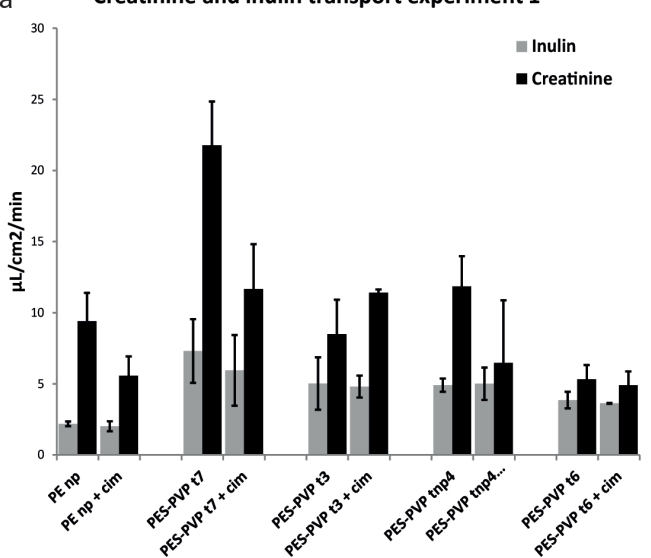

b

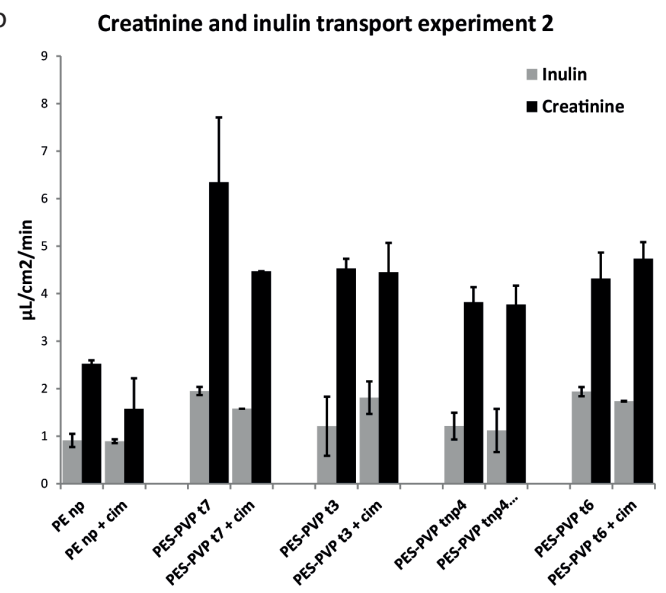

Supplementary Figure 1: Iransport of creatinine and inulin by ciPTEC cultured on porous PES membranes with various

topographies. The graphs show mean inulin and creatinine transport from two experiments. 


\section{Literature}

1. Lovmand J, Justesen J, Foss M, Lauridsen RH, Lovmand M, Modin C, et al. The use of combinatorial topographical libraries for the screening of enhanced osteogenic expression and mineralization. Biomaterials. 2009 Apr;30(11):2015-22. PubMed PMID: 19178942. Epub 2009/01/31. eng.

2. Justesen J, Lorentzen M, Andersen LK, Hansen O, Chevallier J, Modin C, et al. Spatial and temporal changes in the morphology of preosteoblastic cells seeded on microstructured tantalum surfaces. Journal of biomedical materials research Part A. 2009 Jun 15;89(4):885-94. PubMed PMID: 18465820. Epub 2008/05/10. eng.

3. Hoffman-Kim D, Mitchel JA, Bellamkonda RV. Topography, cell response, and nerve regeneration. Annu Rev Biomed Eng. 2010 Aug 15;12:203-31. PubMed PMID: 20438370. Pubmed Central PMCID: PMC3016849. Epub 2010/05/05. eng.

4. Unadkat HV, Hulsman M, Cornelissen K, Papenburg BJ, Truckenmuller RK, Carpenter AE, et al. An algorithmbased topographical biomaterials library to instruct cell fate. Proceedings of the National Academy of Sciences of the United States of America. 2011 Oct 4;108(40):16565-70. PubMed PMID: 21949368. Pubmed Central PMCID: 3189082. Epub 2011/09/29. eng.

5. Saito A, Sawada K, Fujimura S. Present status and future perspectives on the development of bioartificial kidneys for the treatment of acute and chronic renal failure patients. Hemodialysis international International Symposium on Home Hemodialysis. 2011 Apr;15(2):183-92. PubMed PMID: 21395969. Epub 2011/03/15. eng.

6. Buffington DA, Westover AJ, Johnston KA, Humes HD. The bioartificial kidney. Transl Res. 2014 Apr; 163(4):342-51. PubMed PMID: 24269374. Epub 2013/11/26. eng.

7. Humes HD, Buffington D, Westover AJ, Roy S, Fissell WH. The bioartificial kidney: current status and future promise. Pediatr Nephrol. 2014 Mar;29(3):343-51. PubMed PMID: 23619508. Epub 2013/04/27. eng.

8. Ni M, Teo JC, Ibrahim MS, Zhang K, Tasnim F, Chow PY, et al. Characterization of membrane materials and membrane coatings for bioreactor units of bioartificial kidneys. Biomaterials. 2011;32(6):1465-76.

9. Schophuizen CM, De Napoli IE, Jansen J, Teixeira S, Wilmer MJ, Hoenderop JG, et al. Development of a living membrane comprising a functional human renal proximal tubule cell monolayer on polyethersulfone polymeric membrane. Acta biomaterialia. 2015;14:22-32.

10. Fey-Lamprecht F, Gross U, Groth TH, Albrecht W, Paul D, Fromm M, et al. Functionality of MDCK kidney tubular cells on flat polymer membranes for biohybrid kidney. J Mater Sci Mater Med. 1998;9(12):711-5.

11. Fey-Lamprecht F, Groth T, Albrecht W, Paul D, Gross U. Development of membranes for the cultivation of kidney epithelial cells. Biomaterials. 2000;21(2):183-92.

12. Sato $\mathrm{Y}$, Terashima $\mathrm{M}$, Kagiwada $\mathrm{N}$, Tun $\mathrm{T}$, Inagaki $\mathrm{M}$, Kakuta $\mathrm{T}$, et al. Evaluation of proliferation and functional differentiation of LLC-PK1 cells on porous polymer membranes for the development of a bioartificial renal tubule device. Tissue engineering. 2005;11(9-10):1506-15.

13. Kanai N, Fujita Y, Kakuta T, Saito A. The effects of various extracellular matrices on renal cell attachment to polymer surfaces during the development of bioartificial renal tubules. Artificial Organs. 1999 //;23(1):114-8.

14. Zhang H, Tasnim F, Ying JY, Zink D. The impact of extracellular matrix coatings on the performance of human renal cells applied in bioartificial kidneys. Biomaterials. 2009;30(15):2899-911.

15. Fujita Y, Kakuta T, Asano M, Itoh J, Sakabe K, Tokimasa T, et al. Evaluation of Na+ active transport and morphological changes for bioartificial renal tubule cell device using Madin-Darby canine kidney cells. Tissue engineering. 2002;8(1):13-24.

16. IP TK, Aebischer P, Galletti PM. Cellular control of membrane permeability. Implications for a bioartificial renal tubule. ASAIO Transactions. 1988 //;34(3):351-5.

17. van der Aa MA, Helmerhorst TJ, Siesling S, Riemersma S, Coebergh JW. Vaginal and (uncommon) cervical cancers in the Netherlands, 1989-2003. International journal of gynecological cancer : official journal of the International Gynecological Cancer Society. 2010 May;20(4):638-45. PubMed PMID: 20686385. Epub 2010/08/06. eng.

18. Rebollar E, Frischauf I, Olbrich M, Peterbauer T, Hering S, Preiner J, et al. Proliferation of aligned mammalian cells on laser-nanostructured polystyrene. Biomaterials. 2008 Apr;29(12):1796-806. PubMed PMID: 18237776. Epub 2008/02/02. eng. 
19. Frohlich EM, Zhang X, Charest JL. The use of controlled surface topography and flow-induced shear stress to influence renal epithelial cell function. Integr Biol (Camb). 2012 Jan;4(1):75-83. PubMed PMID: 22139064. Epub 2011/12/06. eng.

20. Frohlich EM, Alonso JL, Borenstein JT, Zhang X, Arnaout MA, Charest JL. Topographically-patterned porous membranes in a microfluidic device as an in vitro model of renal reabsorptive barriers. Lab Chip. 2013 Jun 21;13(12):2311-9. PubMed PMID: 23636129. Epub 2013/05/03. eng.

21. Hulsman M, Hulshof F, Unadkat H, Papenburg BJ, Stamatialis DF, Truckenmuller R, et al. Analysis of highthroughput screening reveals the effect of surface topographies on cellular morphology. Acta biomaterialia. 2015 Mar;15:29-38. PubMed PMID: 25554402. Epub 2015/01/03. eng.

22. Charest JL, Garcia AJ, King WP. Myoblast alignment and differentiation on cell culture substrates with microscale topography and model chemistries. Biomaterials. 2007 Apr;28(13):2202-10. PubMed PMID: 17267031. Epub 2007/02/03. eng.

23. Luna JI, Ciriza J, Garcia-Ojeda ME, Kong M, Herren A, Lieu DK, et al. Multiscale biomimetic topography for the alignment of neonatal and embryonic stem cell-derived heart cells. Tissue Eng Part C Methods. 2011 May; 17(5):579-88. PubMed PMID: 21235325. Epub 2011/01/18. eng.

24. Lucker PB, Javaherian S, Soleas JP, Halverson D, Zandstra PW, McGuigan AP. A microgroove patterned multiwell cell culture plate for high-throughput studies of cell alignment. Biotechnol Bioeng. 2014 Dec;111(12): 2537-48. PubMed PMID: 24889796. Epub 2014/06/04. eng.

25. Papenburg BJ, Vogelar L, Bolhuis-Versteeg LA, Lammertink RG, Stamatialis D, Wessling M. One-step fabrication of porous micropatterned scaffolds to control cell behavior. Biomaterials. 2007 Apr;28(11):19982009. PubMed PMID: 17239436. Epub 2007/01/24. eng.

26. Vogelar L, Lammertink RG, Barsema JN, Nijdam W, Bolhuis-Versteeg LA, van Rijn CJ, et al. Phase separation micromolding: a new generic approach for microstructuring various materials. Small. 2005;1(6):645-55.

27. Jansen J, Schophuizen CM, Wilmer MJ, Lahham SH, Mutsaers HA, Wetzels JF, et al. A morphological and functional comparison of proximal tubule cell lines established from human urine and kidney tissue. Experimental cell research. 2014 Apr 15;323(1):87-99. PubMed PMID: 24560744. Epub 2014/02/25. eng.

28. Carpenter AE, Jones TR, Lamprecht MR, Clarke C, Kang IH, Friman 0, et al. CellProfiler: image analysis software for identifying and quantifying cell phenotypes. Genome biology. 2006;7(10):R100. PubMed PMID: 17076895. Pubmed Central PMCID: 1794559. Epub 2006/11/02. eng.

29. Schneider CA, Rasband WS, Eliceiri KW. NIH Image to Image): 25 years of image analysis. Nature methods. 2012 Jul;9(7):671-5. PubMed PMID: 22930834. Epub 2012/08/30. eng.

30. Flemming RG, Murphy CJ, Abrams GA, Goodman SL, Nealey PF. Effects of synthetic micro- and nano- structured surfaces on cell behavior. Biomaterials. 1999 Mar;20(6):573-88. PubMed PMID: 10213360. Epub 1999/04/23. eng.

31. Van Itallie CM, Fanning AS, Bridges A, Anderson JM. 20-1 stabilizes the tight junction solute barrier through coupling to the perijunctional cytoskeleton. Mol Biol Cell. 2009;20(17):3930-40.

32. Denker BM, Sabath E. The biology of epithelial cell tight junctions in the kidney. J Am Soc Nephrol. 2011;22(4):622-5.

33. Zhu LP, YU JZ, XU YY, Xi ZY, Zhu BK. Surface modification of PVDF porous membranes via poly(DOPA) coating and heparin immobilization. Colloids Surf B Biointerfaces. 2009;69(1):152-5.

34. Wilmer MJ, Saleem MA, Masereeuw R, Ni L, van der Velden TJ, Russel FG, et al. Novel conditionally immortalized human proximal tubule cell line expressing functional influx and efflux transporters. Cell and tissue research. 2010 Feb;339(2):449-57. PubMed PMID: 19902259. Pubmed Central PMCID: PMC2817082. Epub 2009/11/11. eng.

35. Schophuizen CM, Wilmer MJ, Jansen J, Gustavsson L, Hilgendorf C, Hoenderop JG, et al. Cationic uremic toxins affect human renal proximal tubule cell functioning through interaction with the organic cation transporter. Pflugers Archiv : European journal of physiology. 2013 Dec;465(12):1701-14. PubMed PMID: 23812163. Epub 2013/07/03. eng.

36. Perrone RD. Means of clinical evaluation of renal disease progression. Kidney international Supplement. 1992 May;36:S26-32. PubMed PMID: 1614064. Epub 1992/05/01. eng. 


\section{CHAPTER 6}

\section{HIGH-THROUGHPUT SCREENING OF CELL INSTRUCTIVE EFFECTS BY DESIGNED NANOSCALE TOPOGRAPHY}

Frits FB Hulshof 1,2, Yiping Zhao ${ }^{3}$, Aliaksei Vasilevich², Nick RM Beijer², Meint de Boer ${ }^{4}$, Bernke J Papenburg³, Clemens van Blitterswijk ${ }^{5}$, Dimitrios Stamatialis ${ }^{1}$, Jan de Boer².

${ }^{1}$ MIRA Institute for Biomedical Technology and Technical Medicine, Department of Biomaterials Science and Technology, University of Maastricht, University of Twente, Enschede, The Netherlands;

${ }^{2}$ MERLN Institute for Technology-Inspired Regenerative Medicine, Department of Cell Biology -inspired Tissue Engineering, Maastricht, The Netherlands;

${ }^{3}$ Materiomics BV, Maastricht, The Netherlands;

${ }^{4} \mathrm{MESA}$ +Institute for Nanotechnology, University of Twente, Enschede, The Netherlands;

${ }^{5}$ MERLN Institute for Technology-Inspired Regenerative Medicine, Department of Complex Tissue Regeneration, University of Maastricht, Maastricht, The Netherlands. 


\section{Abstract}

Surface topography is able to influence cell phenotype in numerous ways and offers opportunities to manipulate cells and tissues. In previous work we have demonstrated the Topochip high-throughput screening platform as a valuable tool to identify bioactive microscale topographies (micro-Topochip) and to infer cell-topography interactions. In this work, we develop the Nano-Topochip on polystyrene (PS) and study the cell instructive effects of nanoscale topographies. A combination of deep UV projection lithography and conventional lithography was used to fabricate a library of more than 1200 different defined nanotopographies in 400 by $400 \mu \mathrm{m}$ test areas separated by $30 \mu \mathrm{m}$ high walls. To investigate the cell instructive effects of nanotopography, actin-RFP labeled U20S osteosarcoma cells were cultured and imaged on the Nano-Topochip. A comparison between U20S cultured on the micro-Topochip and the Nano-TopoChip indicates that the nanoscale topographies mainly affect actin fiber morphology without the cell shape guidance, which occurs on the microtopographies. Subsequent automated image analysis shows that of many cell morphological parameters, cell spreading, cell orientation and actin morphology are most affected by the nanotopographies. Additionally, by using modeling, the changes of cell morphological parameters could by predicted by several feature shape parameters such as lateral size and spacing. This work overcomes the technological challenges of fabricating high quality defined nanoscale features on unprecedented large surface areas of a material relevant for tissue culture such as PS and the screening system is able to infer nanotopography-cell morphological parameter relationships. Our screening platform provides opportunities to identify and study the effect of nanotopography with beneficial properties for the culture of various cell types.

\section{Introduction}

Cells are known to respond to topographical cues of the substrate they come into contact with. Design of surface topography is a strategy to influence the response of cells and tissues to biomaterials. Understanding the underlying mechanisms of cell-topography interactions and applying them for biomaterial design is valuable not only to improve in vitro culture systems (1) but also to improve the interaction of biomedical devices with the human body (2). Microscale topography can strongly affect cellular and nuclear morphology. It can have a strong effect on cell adhesion, the organization of the (nucleo)cytoskeletal system and on fundamental aspects of cell physiology such as differentiation, proliferation, pluripotency and motility (3). Nanoscale topographies are in the size range of filopodia, focal adhesions, lipid rafts, endocytic vesicles and extracellular matrix fibers and may thus affect very different molecular mechanisms than microscale topographies. Molecular events affected are the spacing and clustering of transmembrane adhesion proteins such as integrins that are part of focal adhesion signaling complexes $(4,5)$. This is also supported by the fact that nanotopography affects downstream signaling events such as activation of the integrin-linked kinase/ß-catenin pathway (6). Cells are aligned through the spacing and alignment of focal adhesions by nanoscale wrinkled surfaces (7). Further downstream, nanotopographies affect cell migration (8), proliferation (9) and differentiation $(10,11)$. Additionally, nanotopography can enhance extracellular matrix (ECM) production (12) and may also affect the ECM architecture. Furthermore, nanotopography plays a role in vivo, for example: the surface structure of the basement membrane that interacts with epithelial cell layers (13).

Surface topographies can be fabricated by anisotropic techniques such as sand blasting, acid etching (14) and polymer phase separation (15), but this only gives limited control over the exact dimensions of the surface topographies. Controlled fabrication of designed microscale topographies is possible with microfabrication techniques such as photolithography and deep reactive ion etching (DRIE). Although 
the exact control over the dimensions of the surface topography allows manipulation of aspects of cell morphology, the correlation between cell shape and cell phenotype is largely unknown. Therefore we and others have employed screening approaches to learn about the relationship between topographical design and cellular response $(16,17)$. We previously created the Topochip high-throughput screening platform (18), which allows simultaneous screening of thousands of randomly generated microtopographies. This micro-Topochip contains topographies with height of $10 \mu \mathrm{m}$ and lateral dimensions that range between $5 \mu \mathrm{m}$ to $30 \mu \mathrm{m}$. We have shown that these topographies have significant effects on hMSC differentiation (Chapter 3), maintain pluripotency of induced pluripotent stem cells (1) and kidney epithelial alignment (Chapter 5). Additionally, the screening systems can be used to infer topography design-cell morphology/differentiation relationships (19).

In this work we aim to expand the high-throughput screening system for designed nanoscale topographies, which allows investigation of a library of nanoscale topographies to tap into a new array of cellular mechanisms to influence cell fate. To fabricate such nanotopographies for biological studies, high resolution beam writing techniques such as e-beam or ion-beam lithography are often used, because standard photo-lithography cannot reach the required resolutions. However, compared to lithography, beam writing has the disadvantage of long writing times that are required to cover large areas of multiple $\mathrm{cm}^{2}$, which can negatively affect reproducibility due to beam current fluctuations. These disadvantages severely limit the realistic area size, which can be patterned by these techniques. Systematic investigation into the effect of shape and dimensionality of nanotopography on cell response requires larger substrate areas to be able to measure enough cells to perform statistical analyzes on subtle changes. Advanced lithographic techniques such as Deep UV (DUV) and extreme UV (EUV) lithography are more suitable for the nanopatterning of large areas. While these techniques are being employed in the semi-conductor industry, they are not easily accessible by academic institutions due to their complexity and resulting high cost (20). Here, we use conventional UV lithography and DUV lithography in combination with a custom designed DRIE process to fabricate moulds for nano imprint lithography (NIL) to create the Nano-Topochip. Its design is made by computational pattern generation similar to the micro-Topochip generation algorithm (18). After successful production of the Nano-TopoChip, we first compare the effects of micro and nanotopography on u20S osteosarcoma cells. Subsequently we use bioinformatics tools to identify which cell morphological parameters are affected by the nanotopographies. Finally, the correlations between surface topography design and cell morphological parameters are investigated by predictive modeling to understand how feature dimensions affect cell morphology.

\section{Materials and Methods}

\section{Nano-Topochip design}

Similar to the design of the micro-TopoChip (18), a custom C++ script is used to randomly select 1246 unique topographies from an in silico library, with a theoretical design space of millions of topographies, that was generated by combining the primitive shapes triangle, rectangle and circle. A feature was generated by first randomly selecting parameter values for its size, the number of primitives to be used and the distribution over the different primitive types, the size of the primitives, and the degree to which the primitives were to be aligned. The parameter values were selected within the ranges that are shown in the supplemental table S1. Next, each primitive was placed at a random position inside the feature. Overlapping of primitives was allowed. The script creates a Clewin image file, which was applied on a photomask. The resulting Nano-TopoChip contains a surface of 20x $20 \mathrm{~mm}$ with 1246 unique topography designs in duplo in individual test surfaces of 390 by $390 \mu \mathrm{m}$ called TopoUnits, each separated by $30 \mu \mathrm{m}$ high, 10 
$\mu \mathrm{m}$ wide walls in a 50 by 50 grid. The nano features within each TopoUnit are comprised of primitives of lines, circles and triangles have minimum and maximum lateral dimensions of $200 \mathrm{~nm}$ and $700 \mathrm{~nm}$. Nano features with random shapes are generated with lateral dimensions ranging from $200 \mathrm{~nm}$ to $1000 \mathrm{~nm}$.

\section{Nano-Topochip silicon master-mould fabrication}

Figure 1 illustrates the silicon Nano-Topochip mould fabrication process. Due to the difference of wafer size compatibility between different lithography instruments, the $76 \mathrm{~mm}$ diameter Si wafers were attached to the $100 \mathrm{~mm}$ diameter Si wafers using Fomblin oil during plasma etching processes. Double side polished, <100> oriented, 76 mm diameter Si wafers were used as obtained (Fig. 1a). A bottom anti reflective coating (BARC) layer of $38 \mathrm{~nm}$, a DUV resist layer of $225 \mathrm{~nm}$, and a top anti reflective coating (TARC) layer of $90 \mathrm{~nm}$ were subsequently spin-coated and baked on the Si wafers (Fig. 1b). The exposure was performed by an ASML PAS5500/1100B 100 nm ArF scanner using the conventional exposure mode. After exposure, the resist layer was developed and the TARC layer was removed during development. Detailed information about lithography materials, wafer preparation and exposure settings are proprietary information of ASML (Fig. 1C). Next, the BARC layer was etched using mixed directional ion etching (DRIE, Alcatel AMS100SE Deep RIE system) by $\mathrm{CHF}_{3}$ and Ar flow of $50 \mathrm{sccm}$ respectively, automatic pressure control (APC) of 100\%, inductively coupled plasma (ICP) power of $700 \mathrm{~W}$, capacitively coupled plasma (CCP) power of $20 \mathrm{~W}$ with pulsed low frequency of $80 / 20$ (on/off) ms and electrode temperature of $-20^{\circ} \mathrm{C}$. The etch rate of BARC was approximately $60 \mathrm{~nm} / \mathrm{min}$ and therefore the $38 \mathrm{~nm}$ BARC was removed in $45 \mathrm{~s}$ (Fig. 1d). Si etching was performed using a nano Bosch DRIE (Alcatel AMS100SE Deep RIE system) by SF6 (etching) and $\mathrm{C}_{4} \mathrm{~F}_{8}$ (passivation) flow of $50 \mathrm{sccm}$ respectively and cycle times of $1.5 \mathrm{~s}$ and $0.5 \mathrm{~s}$ respectively. Other settings were kept the same for both gases: APC of 100\% ICP power of $1000 \mathrm{~W}$, CCP power of $30 \mathrm{~W}$ with pulsed low frequency of $10 / 90$ (on/off) ms and electrode temperature of $-40^{\circ} \mathrm{C}$. The etch rates of silicon and resist are approximately $200 \mathrm{~nm} / \mathrm{min}$ and $50 \mathrm{~nm} / \mathrm{min}$ respectively (Fig. 1e). Resist and BARC layers are stripped in $\mathrm{O}_{2}$ plasma (Tepla 300), followed by Piranha cleaning for 15 min $\left(\mathrm{H}_{2} \mathrm{SO}_{4}: \mathrm{H}_{2} \mathrm{O}_{2}=3: 1\right.$, $\mathrm{V} / \mathrm{V}, 96^{\circ} \mathrm{C}$ ). A fluorocarbon (CF) removal step was performed by first dry oxidizing the wafers at $800^{\circ} \mathrm{C}$ for $30 \mathrm{~min}$ followed by oxide removal in 50\% $\mathrm{HF}$ for $1 \mathrm{~min}$ (Fig. 1f). After standard cleaning (fumic $\mathrm{HNO}_{3}$ for $10 \mathrm{~min}$, and $69 \% \mathrm{HNO}_{3}$ at $96^{\circ} \mathrm{C}$ for $10 \mathrm{~min}$ and native oxide removal $1 \% \mathrm{HF}$ for $1 \mathrm{~min}$ ), the wafers were prepared with $600 \mathrm{~nm}$ low pressure chemical vapor deposition (LPCVD) TEOS (silicon oxide formed by decomposing TetraEthylOrthosilicate) followed by annealing at $1150^{\circ} \mathrm{C}$ in nitrogen environment for $3 \mathrm{~h}$ (Fig. 19). Adhesion promoter Hexamethyldisilane (HMDS) (Merck) and positive photo resist Olin 908-12 (Arch Chemicals) was spin-coated on the wafers (Fig. 1h). A chromium mask, created by laser beam pattern generation with a Heidelberg DWL 200, containing $400 \mu \mathrm{m} \times 400 \mu \mathrm{m}$ grids of 10 $\mu \mathrm{m}$ wide was used and the exposure was performed using conventional UV lithography (EVG 620) (Fig. 1i). Pattern transfer from photoresist to annealed TEOS layer was performed by mixed DRIE (Alcatel AMS100DE Deep RIE system) using $\mathrm{C}_{4} \mathrm{~F}_{8^{\prime}}$ He and $\mathrm{CH}_{4}$ flow of $20 \mathrm{sccm}, 150 \mathrm{sccm}$ and $15 \mathrm{sccm}$ respectively, pressure of $8.5 \mathrm{mbar}$, ICP power of $2800 \mathrm{~W}$, CCP power of $350 \mathrm{~W}$ and electrode temperature of $-10^{\circ} \mathrm{C}$. The etch rates of the annealed TEOS and photoresist were approximately $500 \mathrm{~mm} / \mathrm{min}$ and $50 \mathrm{~mm} / \mathrm{min}$ respectively (Fig. 1j). After photoresist removal using $\mathrm{O}_{2}$ plasma and Piranha cleaning (as described in step 6), silicon grids were etched using Alcatel AMS100 SE Deep RIE system by SF ${ }_{6}$ and $\mathrm{O}_{2}$ flow of $100 \mathrm{sccm}$ and $40 \mathrm{sccm}$ respectively, ICP power of $1000 \mathrm{~W}$, CCP of $20 \mathrm{~W}$ with pulsed low frequency of 20/80 (on/off) ms and electrode temperature of $-110^{\circ} \mathrm{C}$ (Fig. 1 k). The etch rates of Si was approximately $4.5 \mu \mathrm{m} / \mathrm{min}$. In the final step, the annealed TEOS layer was removed by etching in 50\% HF for 1 min (Fig. 1l). 
a

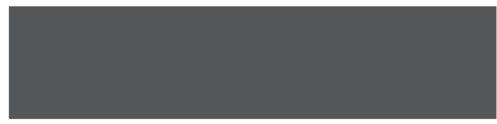

b

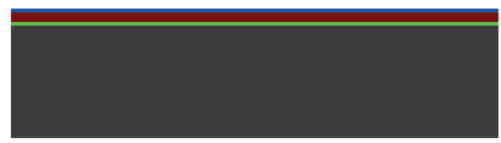

C

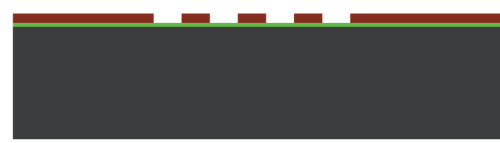

d

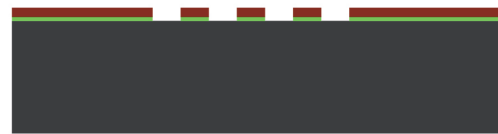

e

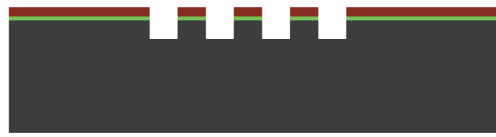

k

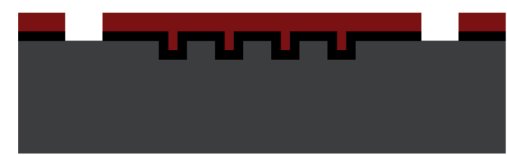

h

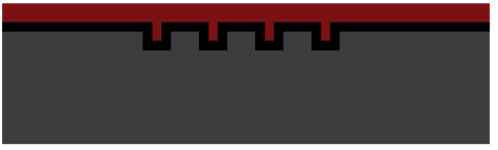

i
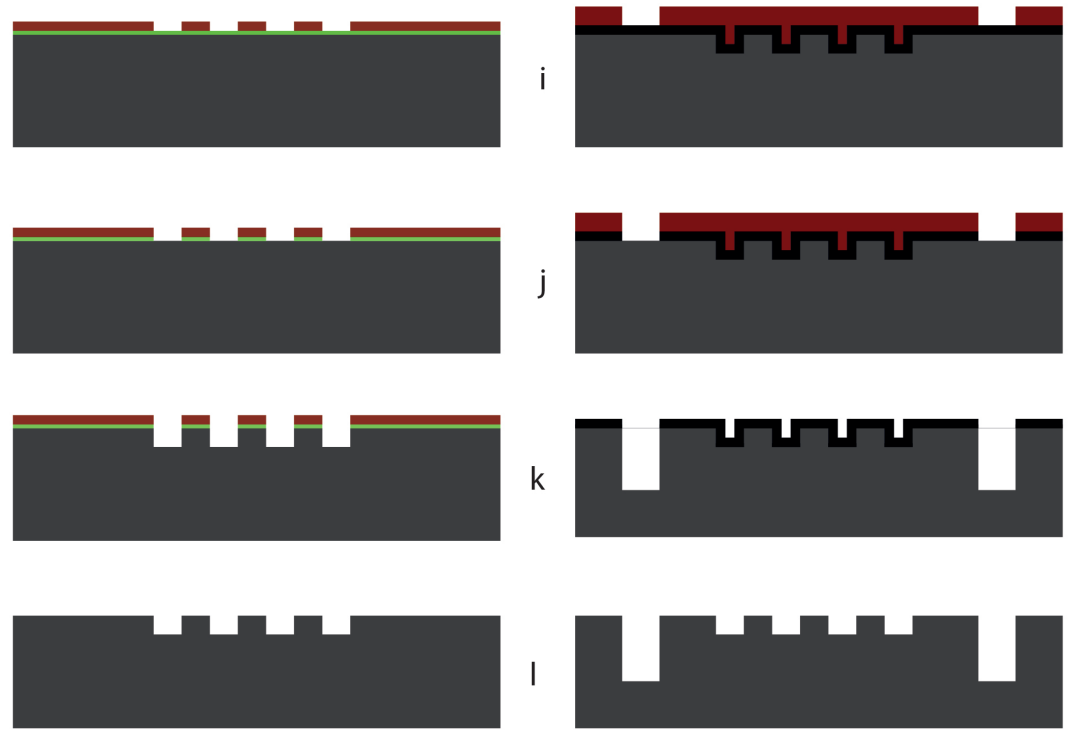

Si

- TARC

- TARF

BARC

- Olin 908-12

- TEOS

Figure 1. Fabrication scheme for lithography and etching of Si Nano-TopoChip mould. The process consists of the following steps (explained in detail in the methods section): Starting Double side polished, <100> oriented, 76 mm diameter Si wafer (a); spin-coating of resists (b); DUV lithography (c); removal of BARC layer (d); Si DRIE (e); resist removal (f); TEOS layer application (g); Spin-coating of photoresist (h); photolithography (i); TEOS DRIE (j); resist removal k; TEOS layer removal (I). 


\section{Ormostamp ${ }^{\circledR}$ mould fabrication.}

Figure 2 depicts the method to fabricate the Ormostamp ${ }^{\circledR}$ mould. The silicon master mould was replicated into Ormostamp moulds in two replication steps. OrmoStamp ${ }^{\circledR}$ (Micro Resist Technology GmbH, Germany) is a UV curable inorganic-organic hybrid polymer and OrmoPrime ${ }^{\circledR} 08$ (Micro Resist Technology $\mathrm{GmbH}$, Germany) is the adhesion promoter for OrmoStamp ${ }^{\circledR}$. Detailed information about these two polymers can be found in the manufacturer's processing protocols. Borofloat wafers (Borofloat ${ }^{\oplus} 33$ from Schott) of $100 \mathrm{~mm}$ diameter and $500 \mu \mathrm{m}$ thickness were used. After Piranha cleaning and dehydration baking on a hot plate at $120^{\circ} \mathrm{C}$ for a minimum of 10 min, OrmoPrime ${ }^{\circledR} 08$ was spin-coated on the Borofloat wafer at $4000 \mathrm{rpm}$ for $30 \mathrm{~s}$ and then baked on a hot plate at $150^{\circ} \mathrm{C}$ for $5 \mathrm{~min}$. In our experiments, a Borofloat wafer with OrmoPrime ${ }^{\circledR} 08$ was prepared right before the application of OrmoStamp ${ }^{\circledR}$ (Fig. 2a). A droplet of $1.5 \mathrm{~mL}$ OrmoStamp ${ }^{\circledR}$ was slowly dispensed on the Si master mould and was slowly brought into contact with the Borofloat wafer with OrmoPrime ${ }^{\circledR} 08$ coating. Slow spreading of the droplet between the Borofloat wafer and Si mould was required to avoid air bubbles getting trapped (Fig. 2b). The gap between the two substrates was completely filled by capillary force, which takes about 15-30 min. (Fig. 2c). The Si and Borofloat wafer stack was exposed to 350-450 nm UV light for $90 \mathrm{~s}$ with the light intensity set at $12 \mathrm{~W} / \mathrm{cm}^{2}$ (EVG 620 i-line exposure system) (Fig. 2d). The Si mould could easily be peeled off from the OrmoStamp ${ }^{\circledR}$ mould, after which the OrmoStamp ${ }^{\circledR}$ mould immediately followed a hard bake process at $130^{\circ} \mathrm{C}$ on a hot plate (ramping up from $20^{\circ} \mathrm{C}$ to $130^{\circ} \mathrm{C}$ with a ramping speed of $5^{\circ} \mathrm{C} / \mathrm{min}$ ). After hard baking for $30 \mathrm{~min}$ at $130^{\circ} \mathrm{C}$, this negative Ormostamp ${ }^{\circledR}$ mould was cooled down together with the hot plate to room temperature (Fig. 2e). Before the second replication step, the OrmoStamp ${ }^{\circledR}$ mould received a gentle $\mathrm{O}_{2}$ plasma treatment by reactive ion etching (RIE, home-build) at $10^{\circ} \mathrm{C}, 50 \mathrm{sccm} \mathrm{O_{2 }}$ flow, 75 mTorr pressure, and $50 \mathrm{~W}$ CCP power for $30 \mathrm{~s}$. After this a monolayer of fluoroctatrichlorosilane (FOTS) was deposited from gas phase under vacuum condition in a desiccator. For the second replication step another borofloat wafer was coated with OrmoPrime ${ }^{\circledR} 08$ as described in 3b (Fig. 2f). As before a droplet of $1.5 \mathrm{~mL}$ OrmoStamp ${ }^{\circledR}$ was slowly dispensed on the negative Ormostamp ${ }^{\circledR}$ mould and is slowly brought into contact with the Borofloat wafer with OrmoPrime ${ }^{\circledR} 08$ coating (Fig. 2g). Again, the gap between the two substrates was filled by capillary force (Fig. 2h). The wafer stack was exposed to UV light (Fig. 2i) after which the negative OrmoStamp ${ }^{\circledR}$ mould could easily be peeled off from the positive OrmoStamp ${ }^{\circledR}$ mould, after hardbaking (Fig. 2j).

\section{Polymer nanochip fabrication}

To prepare the Ormostamp ${ }^{\circledR}$ mould for hot embossing, a gentle $\mathrm{O}_{2}$ plasma treatment was performed using reactive ion etching (RIE, home-build) at $10^{\circ} \mathrm{C}, 50 \mathrm{sccm} \mathrm{O}$ flow, 75 mTorr pressure, and $50 \mathrm{~W}$ CCP power for $30 \mathrm{~s}$. A monolayer of FOTS was deposited from gas phase under vacuum condition in a desiccator (Fig. 2k). Commercially available bi-axially oriented PS films of $190 \mu \mathrm{m}$ (Goodfellow, United Kingdom) were used for hot embossing. PS hot embossing process was performed using the Obducat Eitre ${ }^{\circledR}$ Nano Imprint Lithography system (Obducat, Sweden). The PS film and OrmoStamp ${ }^{\circledR}$ mould were brought into contact (Fig. 2l)) and the hot embossing was performed at a temperature of 140 and a pressure of 10 bar for 5 min (Fig. 2m). Finally, the PS film was separated from the OrmoStamp ${ }^{\circledR}$ mould at $90^{\circ} \mathrm{C}$ (Fig. $2 \mathrm{n}$ ). The Ormostamp moulds ${ }^{\oplus}$ could be used 3 times for hot embossing after which a deterioration of feature and wall quality was observed. 
First replication step

a

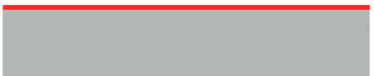

f

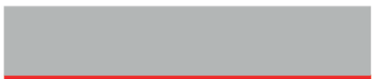

b
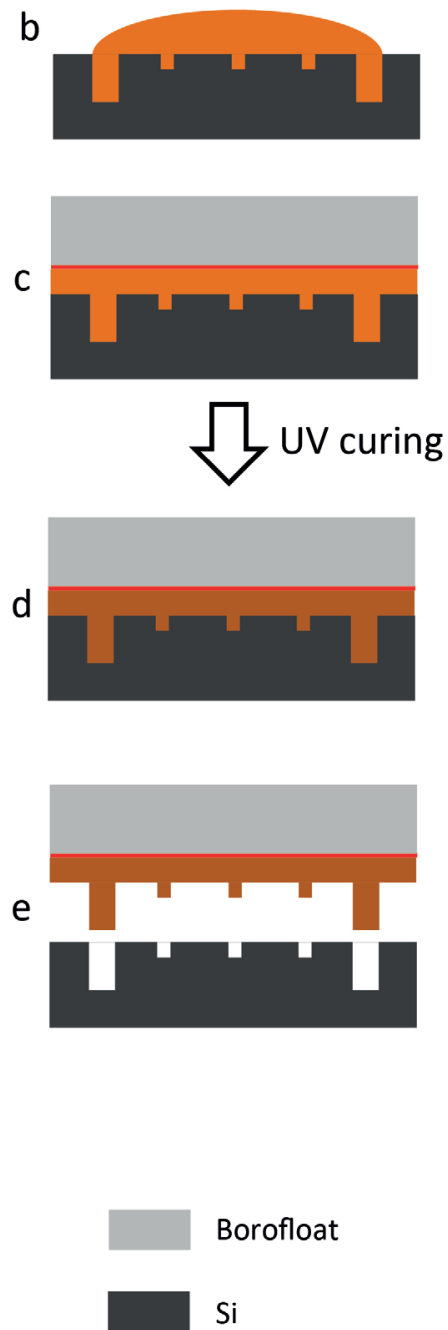

\section{Borofloat}

Si

g
Second replication step
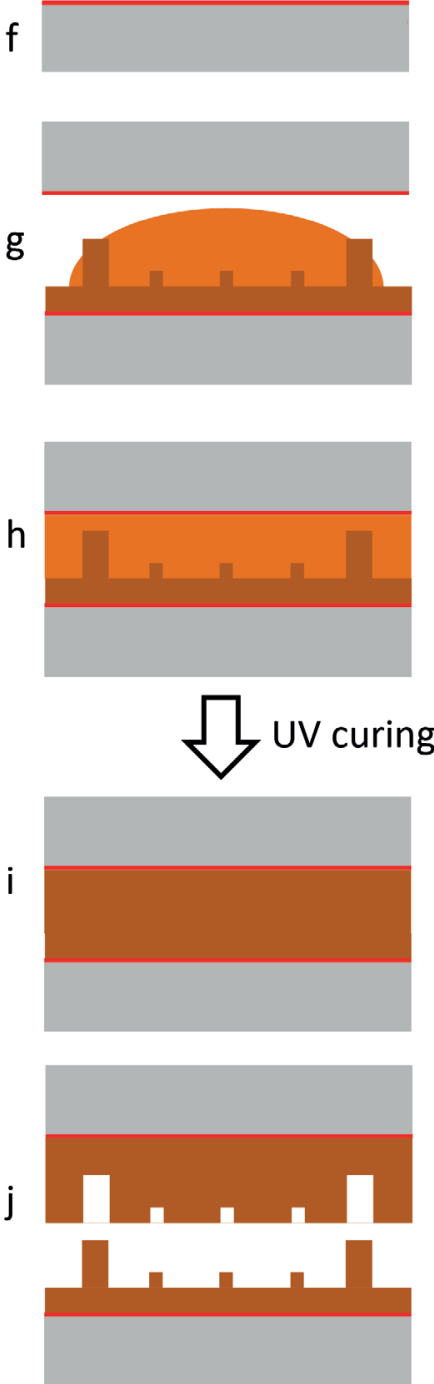

$\mathrm{n}$

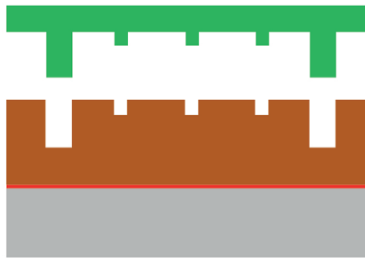

PS hot embossing
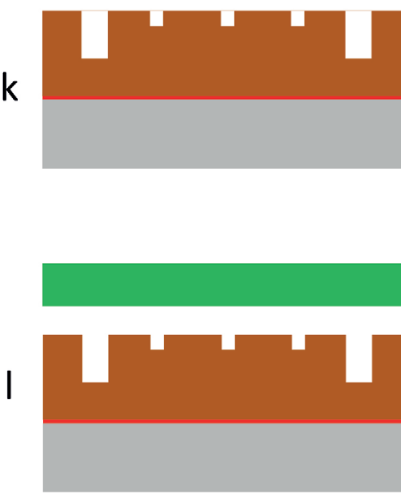

$\mathrm{m}$

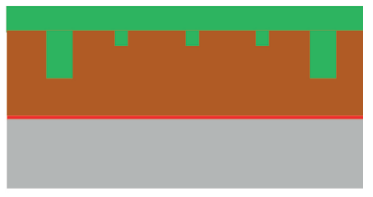

\section{Ormostamp}

Ormoprime 08

\section{Cured Ormostamp}

PS

Figure 2. Fabrication scheme for Ormostamp ${ }^{\circledR}$ mould fabrication and hot embossing of PS Nano-Topochips.

The process to create the first (positive) Ormostamp ${ }^{\circledR}$ mould consisted of the following steps (explained in detail in the methods section): OrmoPrime ${ }^{\oplus 8}$ is applied to a Borofloat ${ }^{\circledR} 33$ (a); application of OrmoStamp ${ }^{\oplus}$ on Si wafer (b); filling by capillary forces (c); UV curing (d); peeling of Ormostamp ${ }^{\circledR}$ mould (e). The second (negative) Ormostamp ${ }^{\circledR}$ mould was created in the following steps: OrmoPrime ${ }^{\circledR 08}$ is applied to a Borofloat ${ }^{\circledR} 33$ (f); application of Ormostamp ${ }^{\circledR}$ on first (positive) OrmoStamp ${ }^{\circledR}$ mould (g); filling by capillary forces (h); uV curing (i); peeling of the second Ormostamp ${ }^{\circledR}$ mould (j). The following steps were performed for the hot embossing process to fabricate the PS Nano-TopoChip: starting situation with the (negative) OrmoStamp ${ }^{\circledR}$ mould (k); application of PS film on the mould (l); hot embossing (m); peeling of NanoTopochip ( $)$. 


\section{Cell culture and imaging}

U20S cells, stably expressing Lamin B1 BFP, ox Tubulin GFP and Actin RFP (U20S LMNB1-TUBA1B-ACTB Sigma-Alldrich) were cultured in McCoys medium (Sigma-Alldrich) with 10\% FBS (Sigma-Alldrich), 100 $\mathrm{U} / \mathrm{ml}$ penicillin and $100 \mathrm{~g} / \mathrm{ml}$ streptomycin (Gibco). The cells were passaged according to manufacturer's protocol. In all experiments, medium was refreshed every two days. For the screening experiment, the cells were seeded at a density of 5000 cells $/ \mathrm{cm}^{2}$ on 6 Nano-TopoChips with a custom built seeding device. The device consists of a culture chamber that fits 2 Nano-TopoChips and creates a small space (500 $\mu \mathrm{m}$ height) on top of the Topochips. The limited space immobilizes the cell suspension by capillary force to achieve homogenous cell seeding for 4 hours after which the lid is removed to allow culture in a conventional volume of medium for adequate nutrient supply and gas exchange. After 3 days of culture, the cells were washed in phosphate buffered (PBS, Sigma Aldrich) and fixated with 1\% paraformaldehyde for 10 mins at $0^{\circ} \mathrm{C}$, followed by quenching with $50 \mathrm{mM}$ ammonium chloride (Sigma Aldrich). Next, the cells were washed with PBS and incubated with 1:10,000 4',6-Diamidino-2-phenylindole (Dapi, Life technologies) for 30 min. Finally the Nano-TopoChips were mounted on coverslips with Mowiol 4-88 (Sigma Aldrich) after washing with PBS twice. After sample drying, the chips were imaged using a Hamamatsu Nanozoomer. A complete image was generated from every Nano-Topochip, which were subsequently cut into smaller images from individual TopoUnits for each separate channel.

\section{Data analysis}

Prior to image analysis, flat-field correction and image normalization were performed as described previously (19). Open source software Cell Profiler (CP) was used for the image analysis (21). In order to perform the automated image analysis in $\mathrm{CP}$, a robust pipeline able to recognize different cell features was built. Data analysis was performed using R, a programming language and software environment for statistical computing and graphics (22). Potential mis-segmentation of cells was detected based on cell area and intensity of the nucleus. The cells were gated based on cell area and perimeter, after which the cells in upper right quadrant were excluded from further analysis (Supplementary fig. S1). To exclude imaging artifacts that could be mistakenly recognized as nuclei, cells were gated based on nuclear mean and integrated intensity, after which the cells in lower left quadrant were excluded from further analysis (Supplementary fig. S2).

For cell profiler features we took the distance the weighted median (23) across all cells in the TopoUnit. For the selected descriptors a rank of surfaces was created based on distance-weighted median calculated from the replicas. For the classification analysis, the top and bottom 100 surfaces were selected. Before training the model we used a recursive feature elimination step to identify features that were important to discriminate these 2 classes. To create the models we used 75\% of the TopoUnits and the accuracy of the model was accessed on the remaining 25\%. The models were trained with 10 fold cross validation in the "caret" package (24). The performance of the model was assessed by the accuracy which is the match between the predicted class, the actual class and thr ROC curve.

\section{Results}

\section{Nano-TopoChip fabrication}

The Nano-TopoChip design was created by computational pattern generation and has an area of $20 \mathrm{~mm}$ 
by $20 \mathrm{~mm}$, which contains 2500 TopoUnits of $390 \mu \mathrm{m}$ by $390 \mu \mathrm{m}$, each separated by $10 \mu \mathrm{m}$ thick walls. The nanotopographies were defined using an ASML PAS5500/1100B of $100 \mathrm{~nm}$ ArF scanner while the wall features were defined by conventional UV lithography. Therefore, a reticle for DUV exposure and a mask for conventional UV lithography were designed. The nanotopographies within the TopoUnits are comprised of primitives of squares, triangles and circles. Considering the maximum resolution of ASML PAS5500/1100B of $100 \mathrm{~nm}$ ArF scanner, the lateral dimension of the features range from $200 \mathrm{~nm}$ to $700 \mathrm{~nm}$. Four Nano-TopoChips with a row and column spacing of $2.4 \mathrm{~mm}$ and $2.56 \mathrm{~mm}$ are scanned on the wafer during Duv exposure. Accordingly, for the walls, grids of $20 \mathrm{~mm}$ by $20 \mathrm{~mm}$ area in the mask for conventional UV lithography were designed sharing the same row and column spacings. Wafers with testing features were first fabricated to test the DRIE BARC and DRIE processes. High resolution scanning electron microscopy (SEM) images of BARC layer before and after etching are shown in Figures $3 \mathrm{a}$ and 3b. The optimized etching processes (described in methods) were used to fabricate the Si mould which is the negative of the Nano-Topochip. The mould contains the $30 \mu \mathrm{m}$ deep trenches (Fig. 3c) to form the walls that will separate the TopoUnits and the nano-seized cavities (Fig. 3d) that will form the nanotopographies. The clearly defined feature shapes are recognizable in the cavities. The silicon moulds were replicated into OrmoStamp ${ }^{\circledR}$ moulds with a two-step process described in the methods section. In summary the silicon mould is first replicated in first (positive) Ormostamp ${ }^{\circledR}$ (Fig. 3e) which is subsequently replicated into a second (negative) Ormostamp (Fig. 3f). Ormostamp ${ }^{\circledR}$ moulds enable hot embossing of polystyrene (PS) films to create PS Nano-Topochips.

To demonstrate differences in dimensions between the features on the micro-Topochip and the Nano-TopoChip an image of the micro-TopoChip (Fig. $3 \mathrm{~g}$ ) is shown below the image of the first Nano-TopoChip Ormostamp ${ }^{\circledR}$ mould (Fig. 3e) of the same magnification.

The second Ormostamp ${ }^{\circledR}$ mould was used to fabricate PS Nano-Topochips by hot embossing. SEM was used for quality inspection of several topographical features of the Nano-TopoChip (Fig. 4). The features have a height of $800 \mathrm{~nm}$. Despite the high aspect ratio of features such as small pillars (Fig. 4ab), the features are stable and the replication is very good without having bending or breakage due to demoulding. Sharp angles of the features are maintained through the replication process. Complex defined shapes are produced with high quality and reproducibility at varying densities. The top of the features is quite flat and display nano-roughness similar to the non-patterned areas.

In conclusion, the Nano-Topochip was produced with excellent quality and reproducibility required for biological screening experiments. We think that the Nano-TopoChip can set a new benchmark for resolution, in comparison to state of the art topographies for biomedical studies, fabricated in PDMS $(17,25,26)$ or PS $(27)$

Figure 3. Quality control of Nano-TopoChip mould fabrication steps. a: SEM images of BARC layer before etching. b: SEM images of BARC layer after etching. SEM images of Nano-Topochip fabrication result. c: A zoom-in image of a nano feature with random contour and smooth bottom and sidewall profile. d: A zoom-out image of nano feature separated by $30 \mu \mathrm{m}$ grid trenches. e: SEM images of the first (positive) Ormostamp mould. f: SEM images of the second (negative) Ormostamp mould used for hot embossing of PS to create the Nano-TopoChips. g: SEM image of a Titanium coated micro-Topochip for size comparison to the first (positive) Ormostamp ${ }^{\circledR}$ mould with the same magnification. 
a

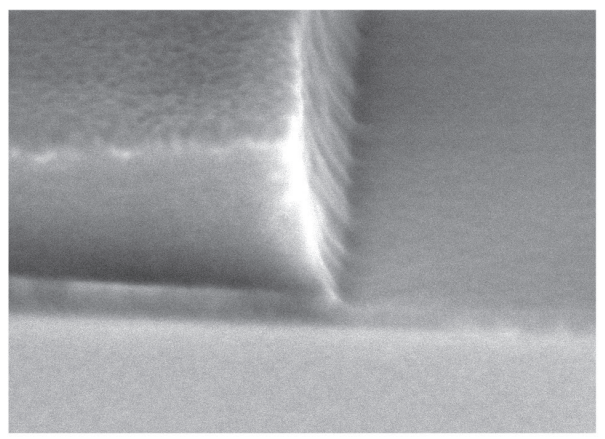

$100 \mathrm{~nm}$

C

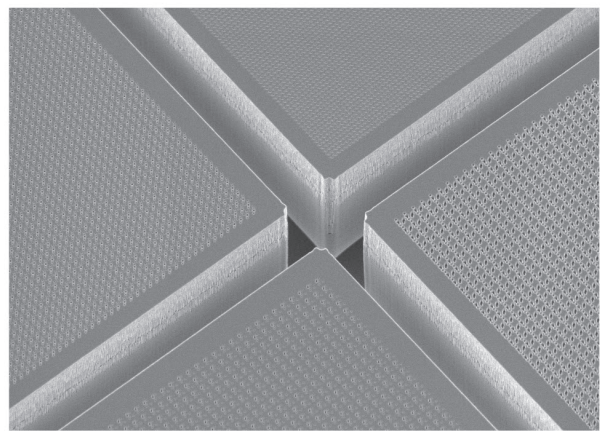

$50 \mu \mathrm{m}$

e

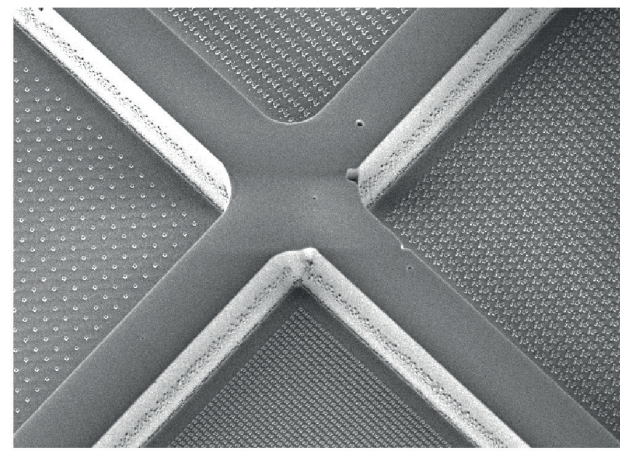

$-10 \mu \mathrm{m}$

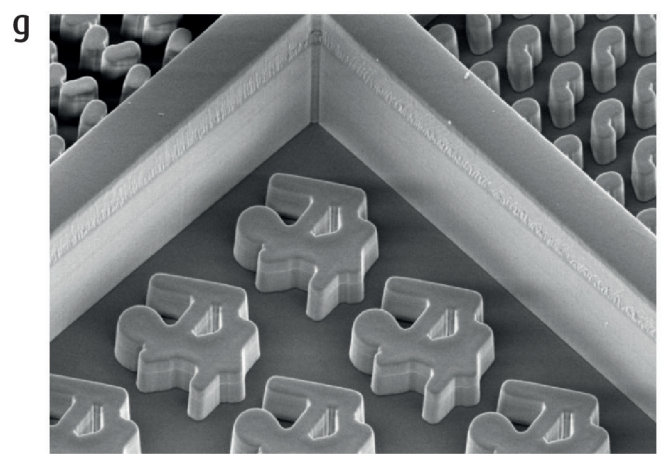

$-10 \mu \mathrm{m}$ b

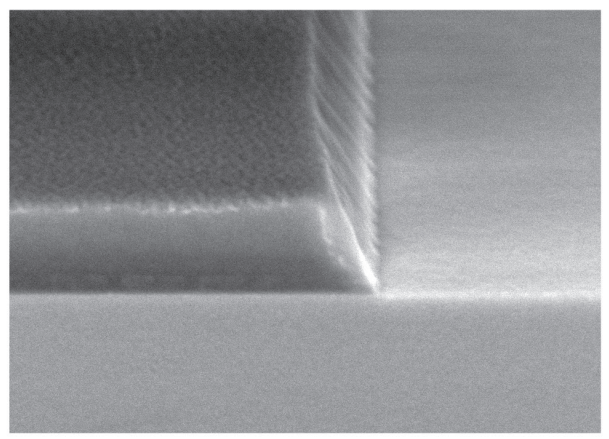

$100 \mathrm{~nm}$

d

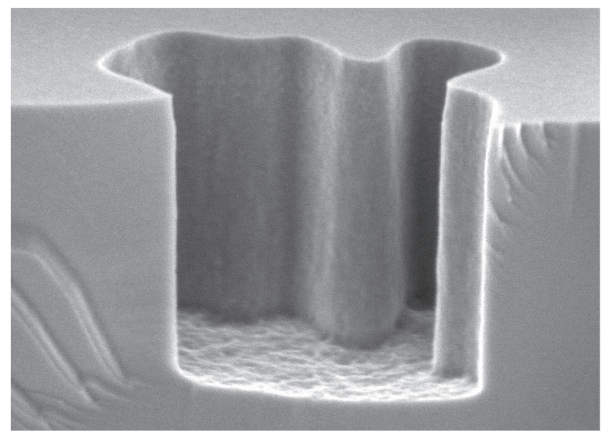

$200 \mathrm{~nm}$

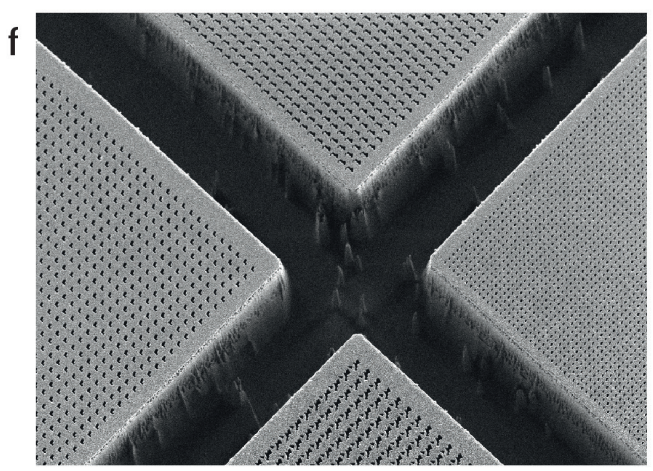

$\longrightarrow 10 \mu \mathrm{m}$ 
a

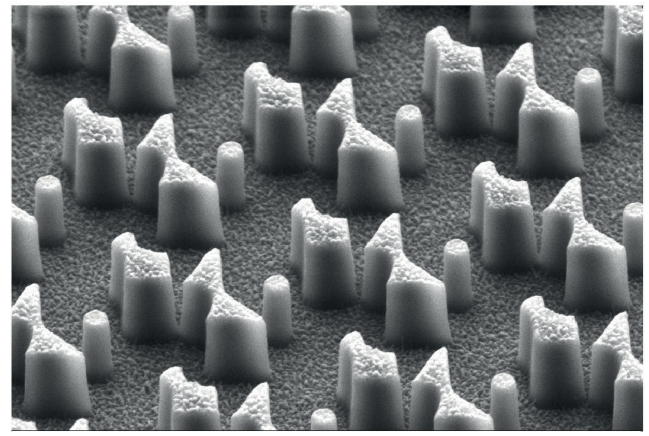

C

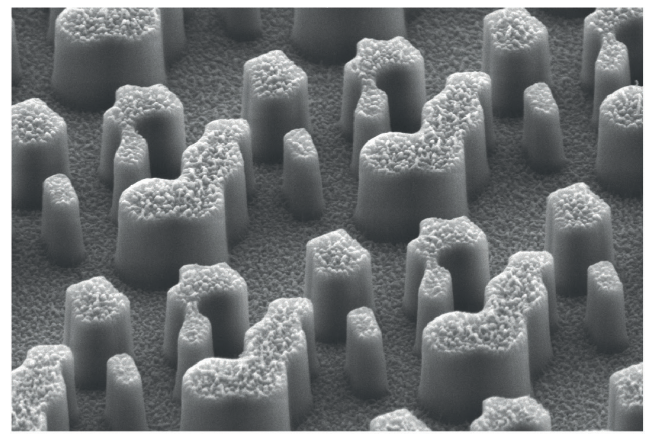

$1 \mu \mathrm{m}$ b

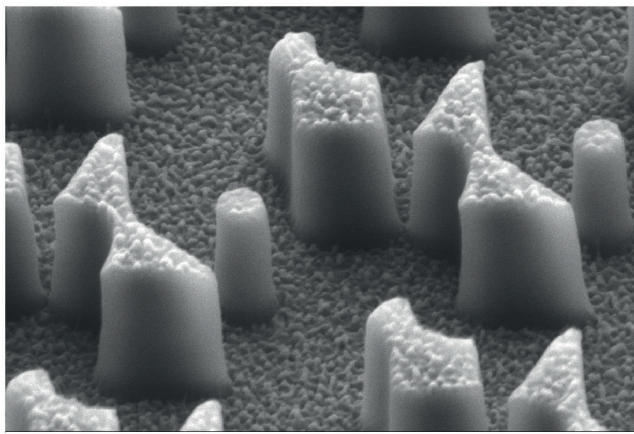

d

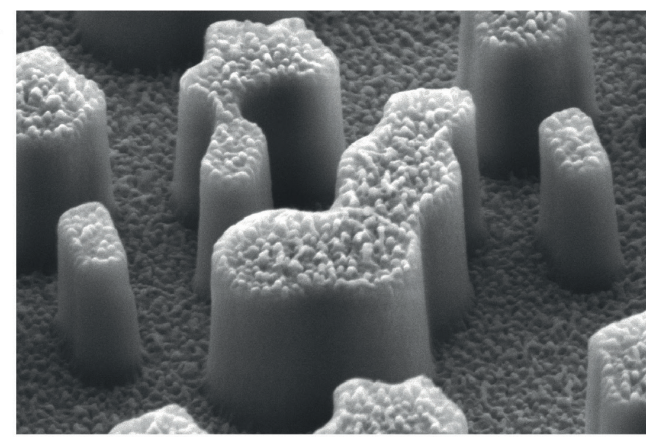

$500 \mathrm{~nm}$

Figure 4: Quality control of the PS Nano-TopoChip. Two topographical features $(a+b, c+d$,) were selected to demonstrate the fabrication quality of nanostructures that have various shapes and densities. Images b and $d$ are higher magnification images of topographies shown in a and c respectively.

\section{U20S Cell morphology on micro-TopoChip and Nano-TopoChip}

To compare the effect of the nanotopographies on cell morphology to the effect of the microtopographies from our current micro-TopoChip, we first performed an experiment with the transgenic U20S cell line, which stably expresses Actin-RFP, cultured on the micro-TopoChip (Fig. 5a). Similar to earlier observations using bone marrow-derived human mesenchymal stromal cells (Chapter 3), U20S cells show a large variety of morphologies on the micro-Topochip. The topographies mould the cells in various shapes and in some cases align the cells into grids (Fig. 5a, image 2). In many cases the actin fibers are combined into thick bundles with a clear orientation directed by the topography (Fig 5a, images 1,2,3). Noteworthy are the extreme nuclear morphologies (Fig. 5a, images 4,5) that are induced by yhe microtopographies. On the microtopographies a classic oval nuclear shape, common on standard culture plastics, is rarely encountered. In fact, the microtopographies induce strong bends in the nuclear membrane and in some cases the nucleus is forced around the features in thin curves shapes (Fig. 5a, image 4). 
a Micro-Topochip

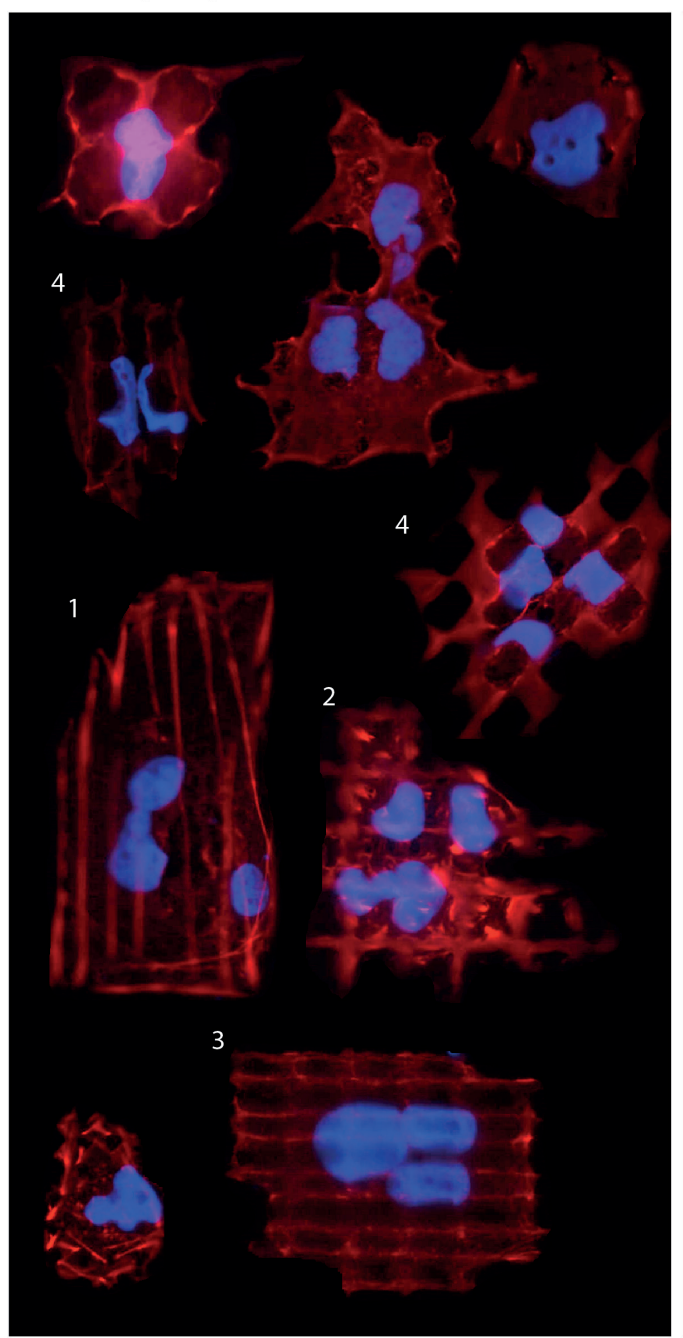

\section{b Nano-TopoChip}

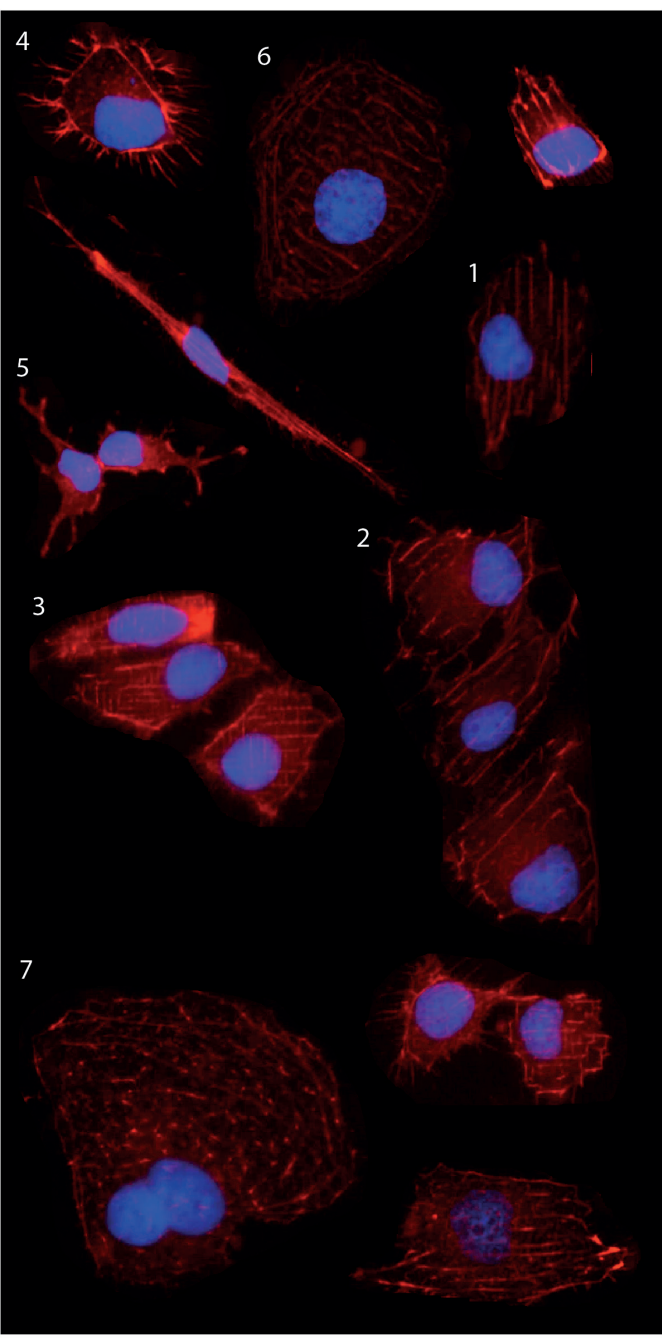

$100 \mu \mathrm{m}$

c non-patterned

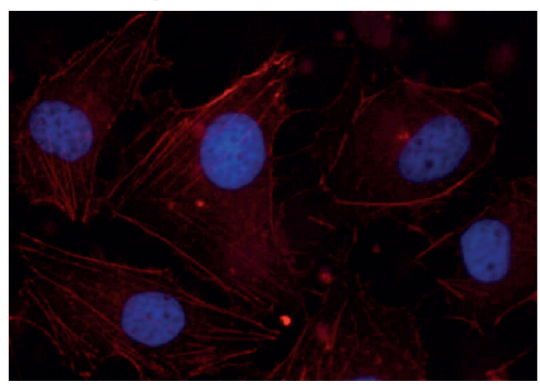

Figure 5: U20S Cell morphology on micro- and Nano-TopoChip.

a: Collage of various morphologies of U2OS cultured on the micro-Topochip.

b: Collage of various morphologies of U2OS cultured on the Nano-Topochip.

c: Image of U2OS cultured on non-patterned surface 
Next, to investigate the effect of nanotopography on cell morphology, u20S cells were cultured on 6 Nano-Topochips for three days. Visual inspection of the images shows that the nanotopographies also affect u20S morphology in various ways and that those are distinctly different from the micro-TopoChip (Fig. 5b). On the Nano-Topochip, we do not observe gross differences in cellular shapes but the nanotopographies seem to affect the extent of cell spreading and the organization of the actin localization. On the nano features, the actin fibers align in various different orientations and patterns (Fig. 5b, images 1,2,3). Interestingly, some features induce the formation of a greater number and larger size filopodia (Fig. 5b, images 4,5), while other nano features create very fine distinctly spaced actin fibers (Fig. 5b 3,6). The more spread cells have very distinct punctate patterns of actin fibers (Fig. 5b, image 7), while the less spread cells tend to have more cortical actin (Fig. 5b, image 4). In this initial observation, the morphology of U2OS nuclei does not seem to deviate much from the common 'classic' oval shape (Fig. 5c).

\section{Nano-Topochip screen}

To quantify the morphological effects of the nanotopographies, we analyzed the images from the 6 Nano-TopoChips using a combination of bioinformatics tools. First, images in the dataset were filtered for outliers caused by events such as focus artifacts and dust particles by using a power log-log slope method (see methods), which might otherwise cause extreme mis-segmentation artifacts.

Next, the images were segmented and parameters such as cell solidity, alignment, number, size and extent were measured by Cell profiler (21), resulting in a dataset for analysis containing 288 (including Metadata) features for each cell. In this experiment, more than 470 thousand cells were measured on a total of 15000 TopoUnits.

To identify the cell features with the highest variation within the whole dataset, a Kruskall-Wallis test was performed on the cell morphology parameters (Fig. 6a). In line with the visual observations the highest variation within the dataset is observed with parameters that determine cell area (Perimeter, Area, Axis Lengths, Diameter) and orientation (Orientation). When we performed the same test on nuclear morphology parameters, we noticed that only the orientation is affected by the topographies (Fig. 6b), whereas there is little effect on the nuclear morphology parameters such as shape and area. This is in line with our visual inspection, which showed that the nuclear morphology not affected by the nanotopographies, especially so when compared to the effect of microtopographies.

To confirm that we can screen for biologically meaningful parameters such as cell spreading, we also quantified the median cell area for every TopoUnit (Fig. 7a). The S-shaped curve shows that the cell area has a normal distribution with a minimum median size of $2460 \mu^{2}$ (17500 pixels) and a maximum median size of $4570 \mu^{2}$ (32500) pixels. The cells on the non-patterned TopoUnits (indicated by the blue dot) have a median size of $3234 \mu^{2}$ (23000 pixels), which is right in the middle of the distribution, showing that a non-patterned surface, on average, leads to a medium area cell size. Representative images of small area cells and large area cells as quantified by Cell profiler is shown in Figure 7b. The actin organization is really different between the large area and small area cells. The small area cells have a large amount densely aligned thin fibers, while the large cells have shorter, thicker fibers with isotropic orientations. 

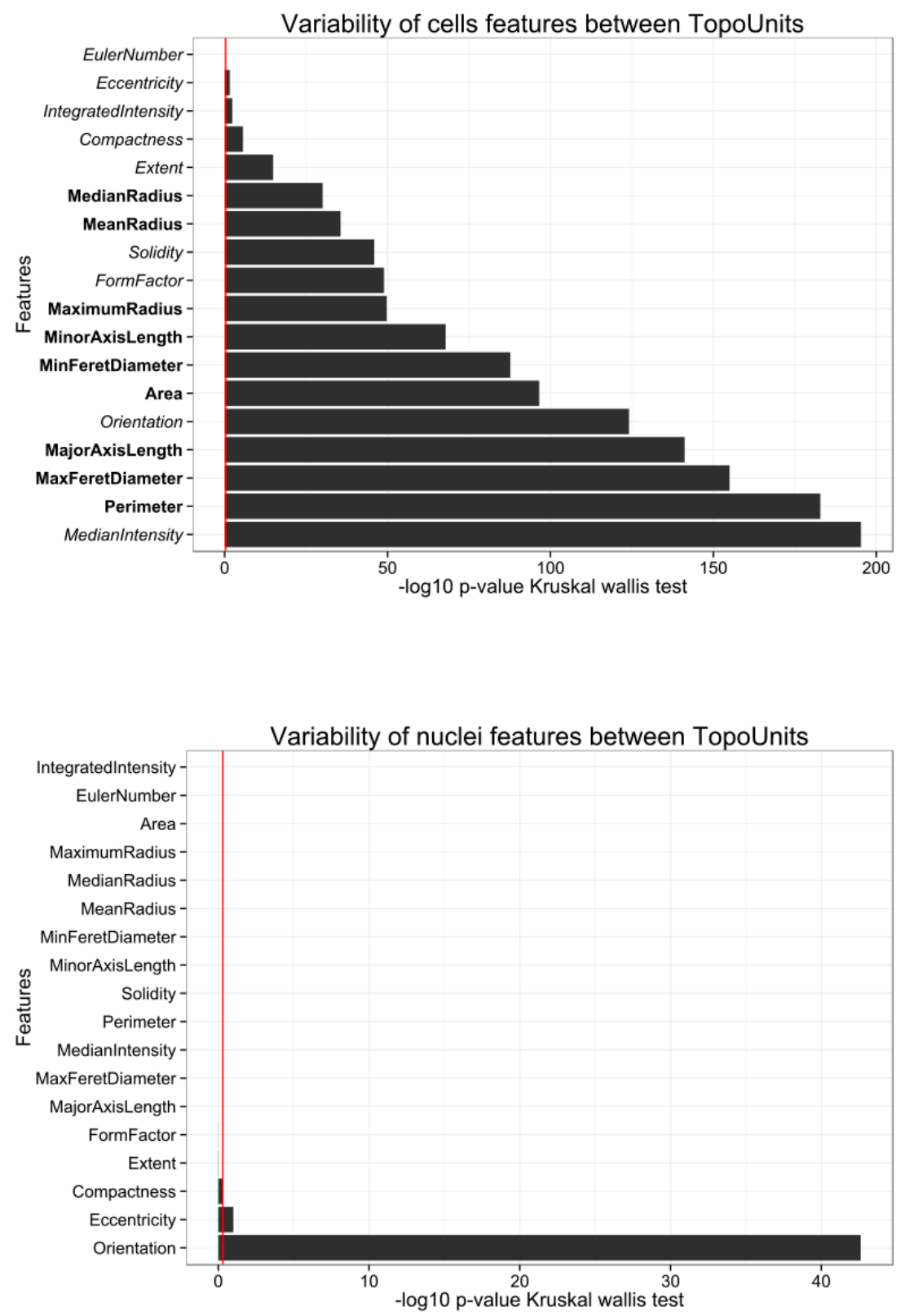

Figure 6: Nanotopographies influence many cell morphological parameters. a: Kruskall-wallis test to identify cell morphological parameters which show the most reproducible variation within the dataset. b: Kruskall-wallis test to identify nuclear morphological parameters which show the most reproducible variation within the dataset. 
To assess if we can accurately predict cell size by surface design parameters, we selected top and bottom ranked surfaces based on cell area as was discussed before (1). We found that random forest was able most accurately predict cell area (Fig. 7c). The area of the smallest individual structure, the 10th percentile of pillars size within the topography and the ratio between area covered by topographical features and the area not covered by topographical features (pattern density) calculated by 2 methods were the most predictive parameters. These surface design parameters were able to predict cell size with 98\% accuracy (Fig. 7d). Visual inspection of the topography designs confirms the model predictions (Fig. 7e). The topographies that induce small, more rounded cells, consist of relatively large topographies with large spacing between the topographies. The topographies which induce more spreading of the cells consist of smaller features which also cover a relatively small area. Other parameters such as cell number (Fig. 8a), actin localization (Fig. 8b) and fiber actin alignment (Fig. 8c) could also be predicted by feature design parameters, using the same methods.

\section{Discussion}

This work shows, for the first time, the fabrication of high quality designed nanometer scale features on a large area such as a $4 \mathrm{~cm}^{2}$ on the Nano-TopoChip, using deep UV projection lithography in combination with conventional lithography (20). Since large surface areas are required to accurately measure events in populations of cells the Nano-TopoChip is an important step towards development of a platform for HTS of events at this scale. In this work we fabricated the Nano-Topochip in PS as it is the golden standard in tissue culture and provides excellent optical properties, which facilitate high-throughput image-based screening experiments. However, one of the major advantages of our fabrication method is that it can be produced in many other different (biomedical) polymers. Surface chemistry strongly dictates cell response (28-30) and may play a critical role in how cells respond to topography.

Comparison of the influence of micro- and nanotopography on cell morphology yielded multiple important observations. The nanotopographies have a limited effect on nuclear morphology which can be extremely affected by micro topographies. The nanotopographies are not able to limit cell volume and shape by moulding the cells between the features such as is often the case with micro topographies. However, they are able to influence cell spreading and actin morphology. In stem cells subtle changes in actin morphology can be correlated to lineage fates (31). By controlling actin morphology we might be able to steer stem cell differentiation.

The machine learning methods used to analyze the large dataset generated in a Nano-Topochip screening experiment help to understand the relationship between nanotopography dimensions and shapes with the cell response. Many cell morphological parameters can be accurately predicted based on a few feature design parameters. With these methods we showed that size and spacing of the nanotopographies have a significant and reproducible effect on cell spreading. The nanotopographies cannot restrict the shape of the cells in contrast to the microscale topographies. This suggests that the nanotopographies must affect the availability and localization of cell attachment sites. Initially we hypothesized that this most likely occurs in an indirect manner, because unlike other studies that are able to restrict integrin binding domain availability by creating binding sites on a non-adhesive substrate (4), cells on the Nano-Topochip are expected to able to bind on the non-patterned areas. The cell attachment to the non-patterned area might be somewhat restricted because of the relatively high aspect ratio on small topographies with little spacing (200 nm width, $800 \mathrm{~nm}$ height), but otherwise the non-patterned areas have the same surface chemistry as the topographical features. Cells bind to serum ECM proteins, such a fibronectin, that adhere to the substrate material (32). It is possible that the nanotopographies influence the alignment of ECM proteins and 
a

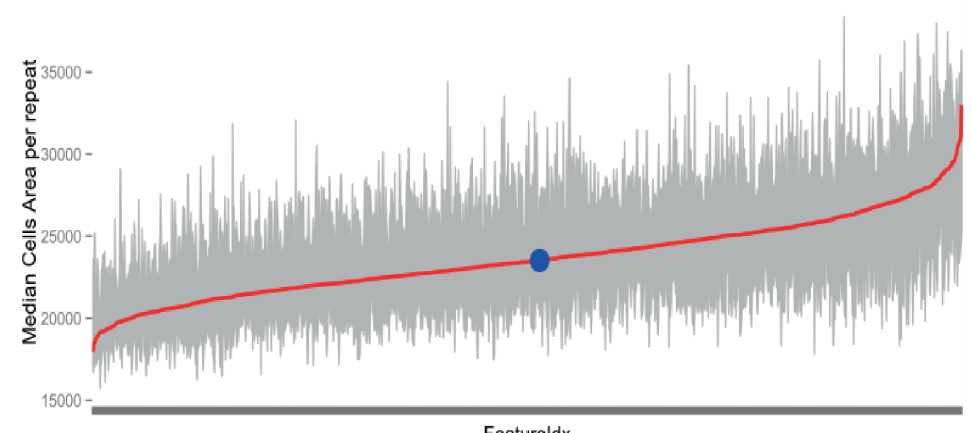

Featureldx

b

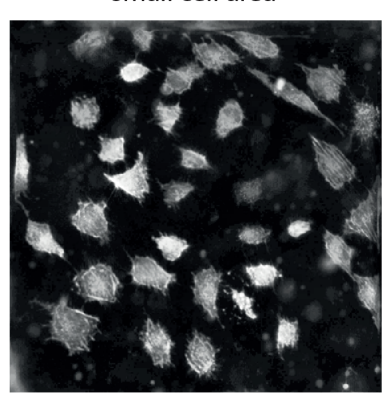

$100 \mu \mathrm{m}$

d

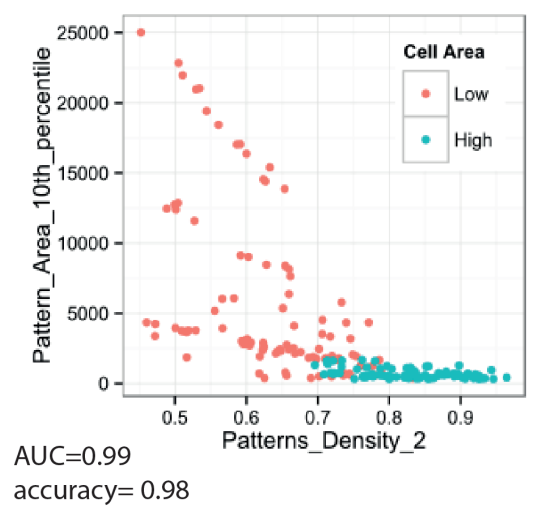

large cell area

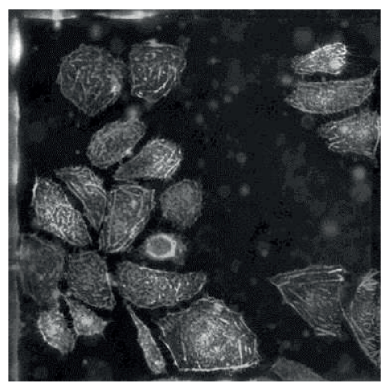

e

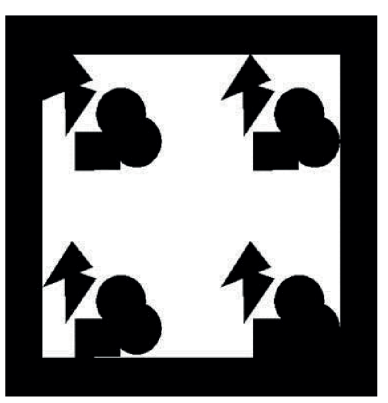

$1 \mu \mathrm{m}$

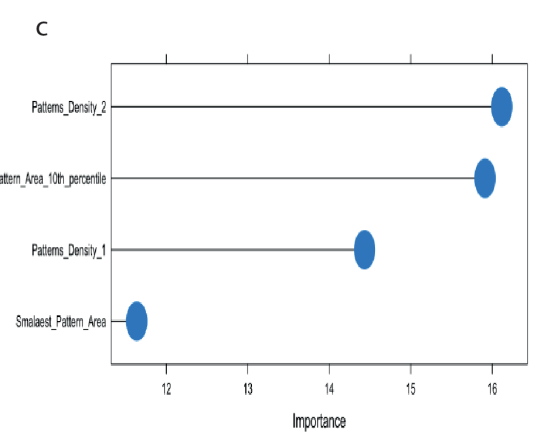

large cell area

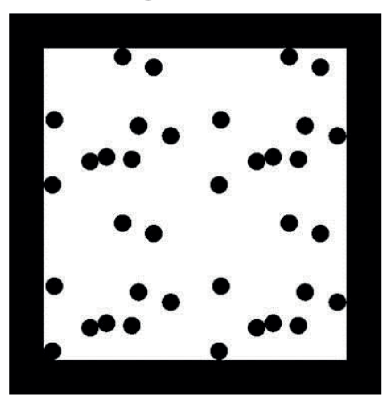

Figure 7: Nanotopographies affect cell area. a: S-curve of Cell area measurement distribution. b: Representative images from the screening experiment which show U2OS with small and large cell areas. c: graph which shows the importance measurements of feature design parameters which influence cell area, by the random forest algorithm. d: Separation of cells with low and high cell area by the '10th percentile area' parameter and the 'pattern density' parameter e: Representative images of features which influence cell area. 
a

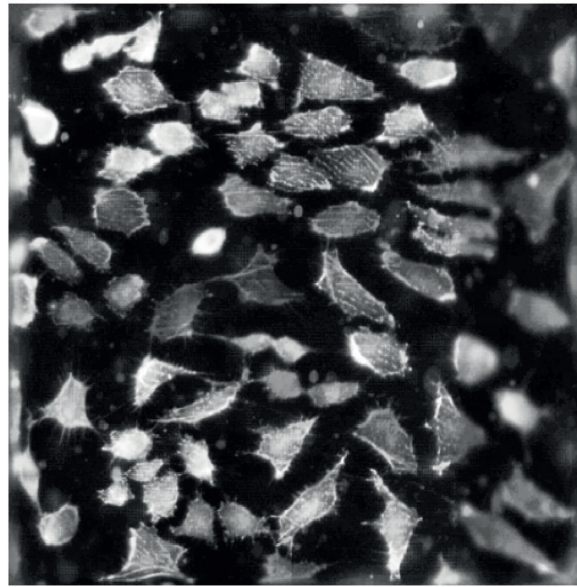

b

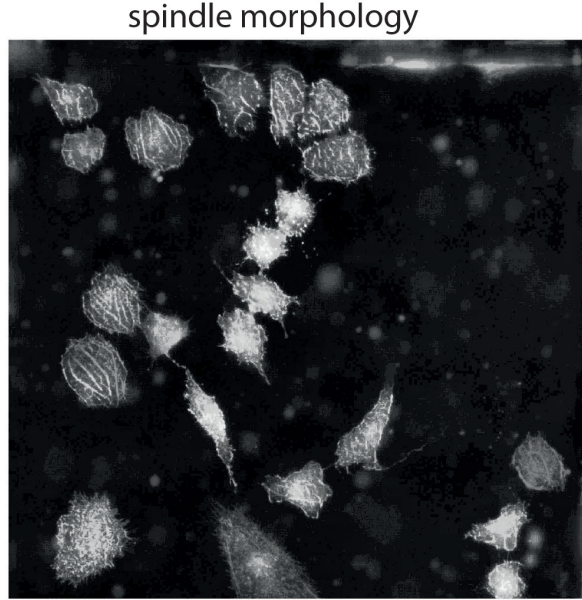

C

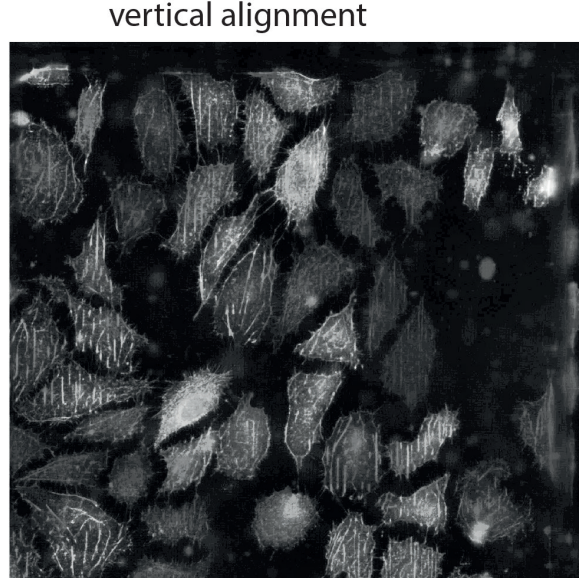

low cell number

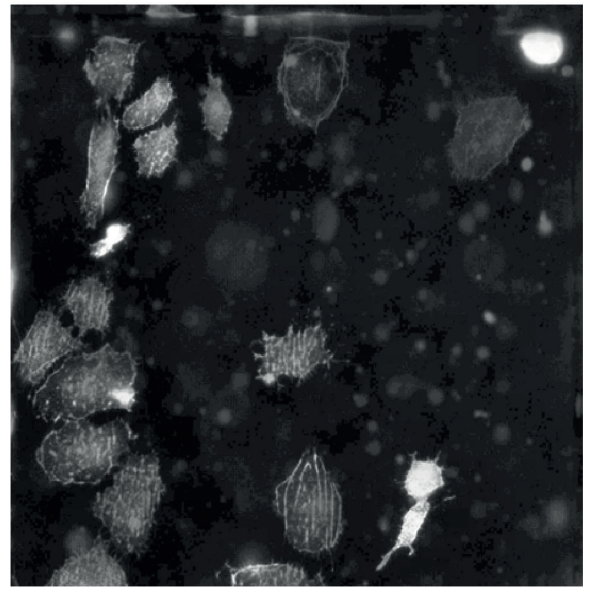

cortical morphology

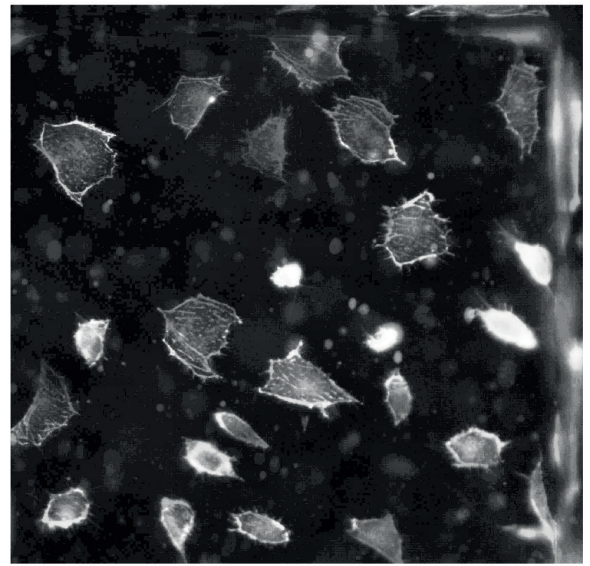

horizontal alignment

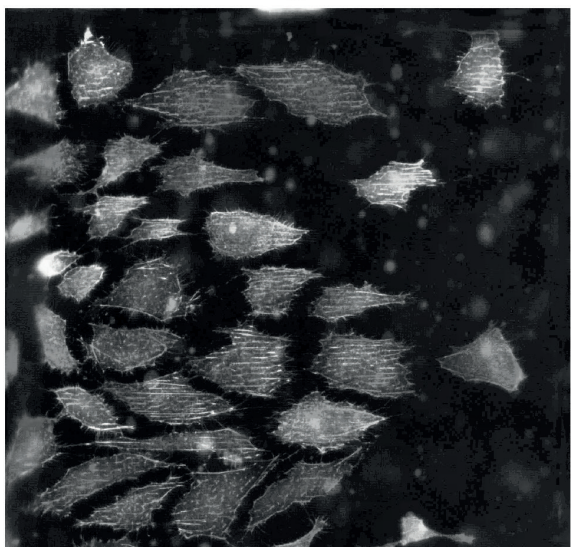

Figure 8: Cell parameters which are affected by nanotopograhy. a: Representative images from the screen of TopoUnits with low and high cell number. b: Representative images from the screen of cells cortical actin localization and actin spindle morphology. c: Representative images from the screen of cells with vertical and horizontal actin fiber orientation. 
In this way, the nanotopographies may indirectly affect binding to cell adhesion molecules such as integrins which are transmembrane proteins that play a key role in cell-ECM interactions (33). Clusters of integrins form the focal adhesion complexes that on the one side attach the cell to matrix proteins while on the other side anchor to the cytoskeleton through adaptor proteins such as talin, paxilin and focal adhesion kinase (34). Even the smallest features are large enough to provide the surface area required for the formation of a small integrin cluster $(4,35)$ required for focal adhesions. Nevertheless, the smallest nano features might limit the size of the integrin clusters. It is suggested that fewer, large integrin clusters result in less cell spreading and thick actin bundles while more, smaller integrin clusters with small spacing leads to more spread cells with smaller focal adhesion sites (36). If the cells in our experiments preferably attach to the top of the topographical features, this would be consistent with our findings which show that fewer large features lead to adhered cells with rounded morphology whereas many small topographical features lead to spread cells.

From these data we can conclude that the cell spreading is affected by the non-patterned area ratio. Although topographies with spread cells tend to have fewer cells, this was not due to space limitation because the cell density in all images was confirmed to be sub confluent. It appears that the fully spread cells have a lower proliferation rate. The integrins in the focal adhesion complexes are mechanically connected to the nucleus through the actin cytoskeleton, and in this way can affect nuclear processes such as transcription and the cell cycle progression (37). This data is quite preliminary. To verify possible effect of cell spreading on proliferation, one would need to repeat the screening experiment with lower initial cell seeding density and longer culture time.

In conclusion, this work showed the fabrication of the Nano-Topochip, which contains a large library of accurately defined, reproducible nanotopographies. Furthermore, a proof of principle screening experiment with U2OS cells demonstrated the Nano-TopoChip high-throughput screening platform as an excellent tool to investigate nanotopography - cell morphological parameter relationships. In future experiments the Nano-Topochip can be used to identify specific nanotopographical designs, which influence functional cell phenotypes such as differentiation states. Interesting candidates for such screening studies are epithelial cell types. Earlier studies have shown that microscale topographies can be very disruptive for epithelial cell layers by reducing cell-cell contacts (Chapter 5). For this reason we expect that cell guidance by surface topography probably works best in the submicron range for epithelial and endothelial cell types that depend on monolayer formation for their polarity, phenotype and function.

Concerning valorization, we think that nanotopographies with beneficial properties could be first applied to tissue culture products if the moulds for hot embossing or injection moulding can be fabricated in a cost effective manner. Later, with the development of new fabrication technologies it might become possible to apply high-resolution topographies to medical devices with complex shapes such as artificial joints.

\section{Acknowledgements}

JdB, DS and FH gratefully acknowledge the financial support of the NanoNext NL program 6c. JdB acknowledges the financial support of the Dutch province of Limburg, AV the European Union's Seventh Framework Program (FP7/2007-2013) (grant agreement 289720). DS acknowledges the financial support by the EU Marie Curie ITN Project BIOART (grant no.316690). FH thanks Mark Smithers from Mesa+ NanoLab for assistance with SEM. Materiomics BV acknowledges Jeroen Bolk, Robert Laar from Nanolab@TU/e, Eindhoven University of Technology, Eindhoven, The Netherlands. 


\section{Supplementary data}

Table S1. Overview of parameter values and ranges used to construct features

\begin{tabular}{|ll|}
\hline Parameter & Value or range \\
\hline Feature space side length $10 \mu \mathrm{m}, 20 \mu \mathrm{m}$, or $28 \mu \mathrm{m}$ & $1 \mu \mathrm{m}, 1.8 \mu \mathrm{m}, 2,4 \mu \mathrm{m}$ \\
\hline No. of primitives used $(1 \mu \mathrm{m})$ & $3-4$ \\
\hline No. of primitives used $(1.8 \mu \mathrm{m})$ & $3-8$ \\
\hline No. of primitives used $(2,4 \mu \mathrm{m})$ & $3-12$ \\
\hline Diameter of a circle primitive $(1 \mu \mathrm{m})$ & $200 \mathrm{~nm}-300 \mathrm{~nm}$ \\
\hline Diameter of a circle primitive $(1,8 \mu \mathrm{m})$ & $200 \mathrm{~nm}-500 \mathrm{~nm}$ \\
\hline Diameter of a circle primitive $(2,4 \mu \mathrm{m})$ & $200 \mathrm{~nm}-700 \mathrm{~nm}$ \\
\hline Shortest side length of a triangle primitive & $200 \mathrm{~nm}-300 \mathrm{~nm}$ \\
\hline Shortest side length of a triangle primitive & $200 \mathrm{~nm}-500 \mathrm{~nm}$ \\
\hline Shortest side length of a triangle primitive & $200 \mathrm{~nm}-700 \mathrm{~nm}$ \\
\hline Top angle of a triangle primitive & $36^{\circ}$ \\
\hline Length of a line primitive & $200 \mathrm{~nm}-300 \mathrm{~nm}$ \\
\hline Length of a line primitive & $200 \mathrm{~nm}-500 \mathrm{~nm}$ \\
\hline Length of a line primitive & $200 \mathrm{~nm}-700 \mathrm{~nm}$ \\
\hline Thickness of a line primitive & $200 \mathrm{~nm}$ \\
\hline Standard deviation for the rotation of a primitive & $0.0^{\circ}-180.0^{\circ}$ \\
\hline
\end{tabular}




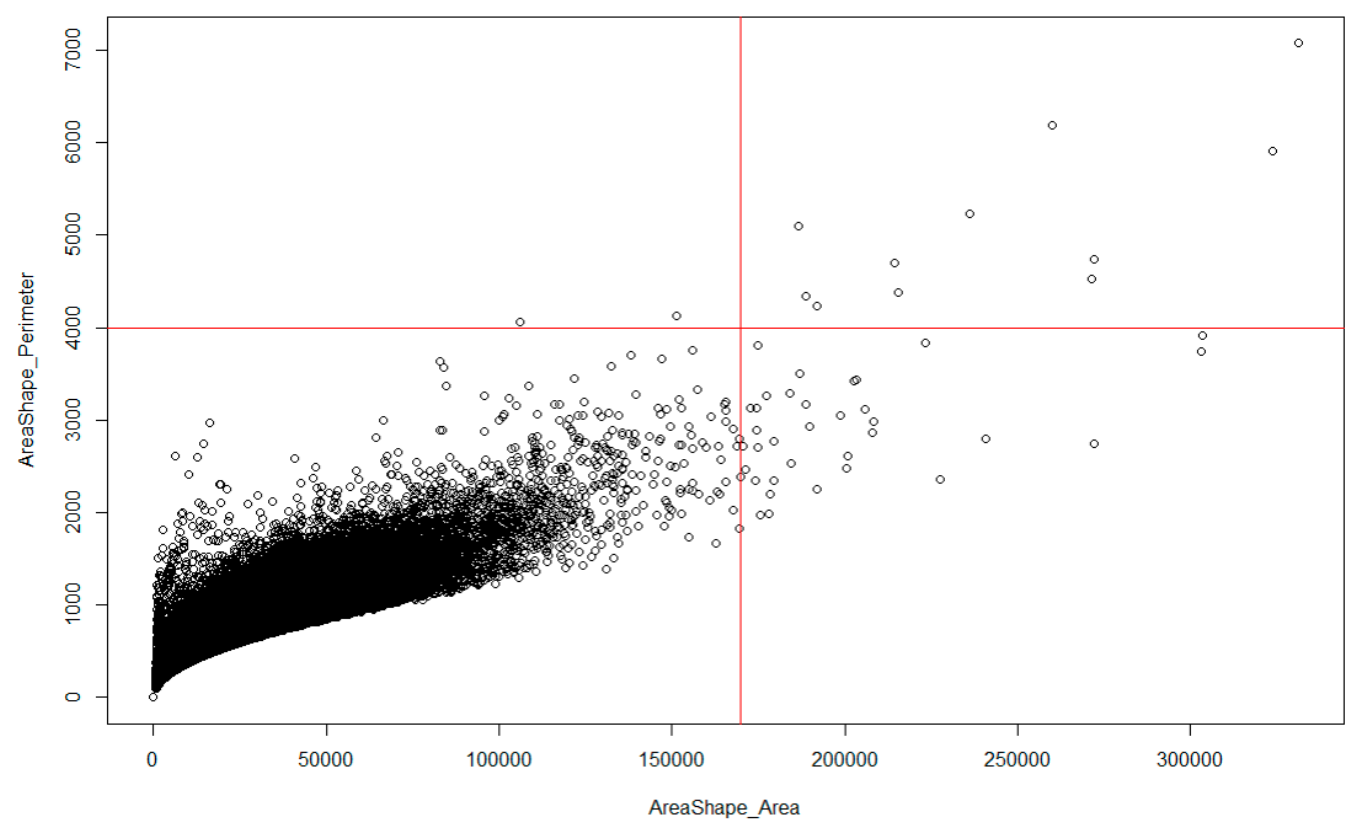

Supplementary figure 1: Outlier removal caused by mis-segmentation. Cells were gated based on cell area and perimeter and cells in upper right quadrant were excluded from further analysis.

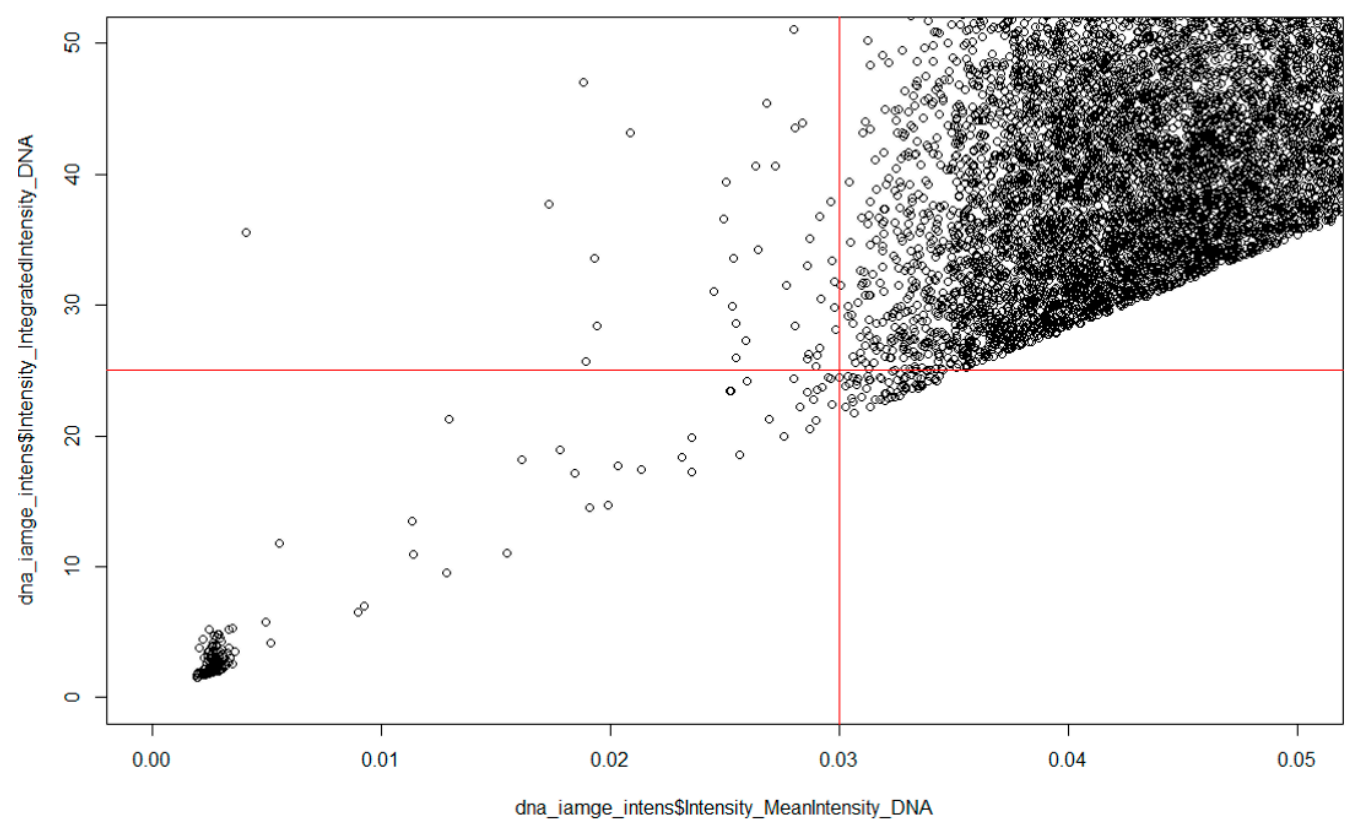

Supplementary figure 2: Outlier removal caused by imaging artifacts. Cells were gated based on nuclei mean and integrated intensity and cells in lower left quadrant were excluded from further analysis 


\section{Literature}

1. Reimer A, Vasilevich A, Hulshof F, Viswanathan P, van Blitterswijk CA, de Boer J, et al. Scalable topographies to support proliferation and Oct4 expression by human induced pluripotent stem cells. Scientific reports. 2016;6:18948. PubMed PMID: 26757610. Epub 2016/01/14. eng.

2. Arlin ML. Survival and success of sandblasted, large-grit, acid-etched and titanium plasma-sprayed implants: a retrospective study. Journal (Canadian Dental Association). 2007 Nov;73(9):821. PubMed PMID: 18028757. Epub 2007/11/22. eng.

3. Makhija E, Jokhun DS, Shivashankar GV. Nuclear deformability and telomere dynamics are regulated by cell geometric constraints. Proceedings of the National Academy of Sciences of the United States of America. 2016 Jan 5;113(1):E32-40. PubMed PMID: 26699462. Epub 2015/12/25. eng.

4. Schvartzman M, Palma M, Sable J, Abramson J, Hu X, Sheetz MP, et al. Nanolithographic control of the spatial organization of cellular adhesion receptors at the single-molecule level. Nano letters. 2011 Mar 9;11(3): 1306-12. PubMed PMID: 21319842. Pubmed Central PMCID: PMC3061283. Epub 2011/02/16. eng.

5. Cavalcanti-Adam EA, Volberg T, Micoulet A, Kessler H, Geiger B, Spatz JP. Cell spreading and focal adhesion dynamics are regulated by spacing of integrin ligands. Biophysical journal. 2007 Apr 15;92(8):2964-74. PubMed PMID: 17277192. Pubmed Central PMCID: PMC1831685. Epub 2007/02/06. eng.

6. Wang W, Zhao L, Wu K, Ma Q, Mei S, Chu PK, et al. The role of integrin-linked kinase/beta-catenin pathway in the enhanced MG63 differentiation by micro/nano-textured topography. Biomaterials. 2013 Jan;34(3): 631-40. PubMed PMID: 23107296. Epub 2012/10/31. eng.

7. Zhou Q, Kuhn PT, Huisman T, Nieboer E, van Zwol C, van Kooten TG, et al. Directional nanotopographic gradients: a high-throughput screening platform for cell contact guidance. Scientific reports. 2015;5:16240. PubMed PMID: 26572879. Pubmed Central PMCID: PMC4647116. Epub 2015/11/18. eng.

8. Diehl KA, Foley JD, Nealey PF, Murphy CJ. Nanoscale topography modulates corneal epithelial cell migration. Journal of biomedical materials research Part A. 2005 Dec 1;75(3):603-11. PubMed PMID: 16106433. Epub 2005/08/18. eng.

9. Park J, Bauer S, Schlegel KA, Neukam FW, von der Mark K, Schmuki P. TiO2 nanotube surfaces: 15 nm-an optimal length scale of surface topography for cell adhesion and differentiation. Small (Weinheim an der Bergstrasse, Germany). 2009 Mar;5(6):666-71. PubMed PMID: 19235196. Epub 2009/02/24. eng.

10. de Peppo GM, Agheli H, Karlsson C, Ekstrom K, Brisby H, Lenneras M, et al. Osteogenic response of human mesenchymal stem cells to well-defined nanoscale topography in vitro. International journal of nanomedicine. 2014;9:2499-515. PubMed PMID: 24904210. Pubmed Central PMCID: PMC4039423. Epub 2014/06/07. eng.

11. Dalby MJ, Gadegaard N, Tare R, Andar A, Riehle MO, Herzyk P, et al. The control of human mesenchymal cell differentiation using nanoscale symmetry and disorder. Nature materials. 2007 Dec;6(12):997-1003. PubMed PMID: 17891143. Epub 2007/09/25. eng.

12. Yamada M, Kato E, Yamamoto A, Sakurai K. A titanium surface with nano-ordered spikes and pores enhances human dermal fibroblastic extracellular matrix production and integration of collagen fibers. Biomedical materials (Bristol, England). 2016;11(1):015010. PubMed PMID: 26835848. Epub 2016/02/03. eng.

13. Garland SP, McKee CT, Chang YR, Raghunathan VK, Russell P, Murphy CJ. A cell culture substrate with biologically relevant size-scale topography and compliance of the basement membrane. Langmuir. 2014;30(8):2101-8.

14. Zhao G, Schwartz Z, Wieland M, Rupp F, Geis-Gerstorfer J, Cochran DL, et al. High surface energy enhances cell response to titanium substrate microstructure. Journal of biomedical materials research Part A. 2005 Jul 1;74(1):49-58. PubMed PMID: 15924300. Epub 2005/06/01. eng.

15. Maclaine SE, Gadhari N, Pugin R, Meek RM, Liley M, Dalby MJ. Optimizing the osteogenicity of nanotopography using block co-polymer phase separation fabrication techniques. Journal of orthopaedic research: official publication of the Orthopaedic Research Society. 2012 Aug;30(8):1190-7. PubMed PMID: 22294345. Epub 2012/02/02. eng. 
16. Kolind K, Dolatshahi-Pirouz A, Lovmand J, Pedersen FS, Foss M, Besenbacher F. A combinatorial screening of human fibroblast responses on microstructured surfaces. Biomaterials. 2010 Dec;31(35):9182-91. PubMed PMID: 20832853. Epub 2010/09/14. eng.

17. Moe AA, Suryana M, Marcy G, Lim SK, Ankam S, Goh JZ, et al. Microarray with micro- and nanotopographies enables identification of the optimal topography for directing the differentiation of primary murine neural progenitor cells. Small (Weinheim an der Bergstrasse, Germany). 2012 Oct 8;8(19):3050-61. PubMed PMID: 22807278. Epub 2012/07/19. eng.

18. Unadkat HV, Hulsman M, Cornelissen K, Papenburg BJ, Truckenmuller RK, Carpenter AE, et al. An algorithm-based topographical biomaterials library to instruct cell fate. Proceedings of the National Academy of Sciences of the United States of America. 2011 Oct 4;108(40):16565-70. PubMed PMID: 21949368. Pubmed Central PMCID: 3189082. Epub 2011/09/29. eng.

19. Hulsman M, Hulshof F, Unadkat H, Papenburg BJ, Stamatialis DF, Truckenmuller R, et al. Analysis of highthroughput screening reveals the effect of surface topographies on cellular morphology. Acta biomaterialia. 2015 Mar;15:29-38. PubMed PMID: 25554402. Epub 2015/01/03. eng.

20. Chen W, Shao Y, Li X, Zhao G, Fu J. Nanotopographical Surfaces for Stem Cell Fate Control: Engineering Mechanobiology from the Bottom. Nano today. 2014 Dec 1;9(6):759-84. PubMed PMID: 25883674. Pubmed Central PMCID: PMC4394389. Epub 2015/04/18. Eng.

21. Carpenter AE, Jones TR, Lamprecht MR, Clarke C, Kang IH, Friman 0, et al. CellProfiler: image analysis software for identifying and quantifying cell phenotypes. Genome Biol. 2006;7(10):R100. PubMed PMID: 17076895. Pubmed Central PMCID: 1794559. Epub 2006/11/02. eng.

22. Team RDC. R: A Language and Environment for Statistical Computing. the R Foundation for Statistical Computing http://wwwR-projectorg/. 2011.

23. Dodonov Y DY. Distance-weighted statistics: robust estimation of location, spread and association between variables. 2013.

24. Kuhn M. Building Predictive Models in R Using the caret Package. 2008. 2008 2008-11-10;28(5):26. Epub 2008-09-23.

25. Eichhorn M, Stannard C, Anselme K, Ruhe J. Nucleus deformation of SaOs-2 cells on rhombic micro-pillars. J Mater Sci Mater Med. 2015 Feb;26(2):108. PubMed PMID: 25665842. Epub 2015/02/11. eng.

26. Li W, Tang QY, Jadhav AD, Narang A, Qian WX, Shi P, et al. Large-scale Topographical Screen for Investigation of Physical Neural-Guidance Cues. Scientific reports. 2015 03/02 10/22/received01/29/accepted;5:8644. PubMed PMID: PMC4345323.

27. Muhammad R, Peh GS, Adnan K, Law JB, Mehta JS, Yim EK. Micro- and nanotopographyto enhance proliferation and sustain functional markers of donor-derived primary human corneal endothelial cells. Acta biomaterialia. 2015 Jun;19:138-48. PubMed PMID: 25796353. Epub 2015/03/23. eng.

28. Brodbeck WG, Nakayama Y, Matsuda T, Colton E, Ziats NP, Anderson JM. Biomaterial surface chemistry dictates adherent monocyte/macrophage cytokine expression in vitro. Cytokine. 2002 Jun 21;18(6):311-9. PubMed PMID: 12160519. Epub 2002/08/06. eng.

29. Shadanbaz S, Dias G). Calcium phosphate coatings on magnesium alloys for biomedical applications: a review. Acta biomaterialia. 2012 Jan;8(1):20-30. PubMed PMID: 22040686. Epub 2011/11/02. eng.

30. Mei Y, Saha K, Bogatyrev SR, Yang J, Hook AL, Kalcioglu ZI, et al. Combinatorial development of biomaterials for clonal growth of human pluripotent stem cells. Nature materials. 2010 Sep;9(9):768-78. PubMed PMID: 20729850. Pubmed Central PMCID: PMC3388774. Epub 2010/08/24. eng.

31. Treiser MD, Yang EH, Gordonov S, Cohen DM, Androulakis IP, Kohn J, et al. Cytoskeleton-based forecasting of stem cell lineage fates. Proceedings of the National Academy of Sciences of the United States of America. 2010 Jan 12;107(2):610-5. PubMed PMID: 20080726. Pubmed Central PMCID: PMC2818905. Epub 2010/01/19. eng.

32. Sethuraman A, Han M, Kane RS, Belfort G. Effect of surface wettability on the adhesion of proteins. Langmuir. 2004 Aug 31;20(18):7779-88. PubMed PMID: 15323531. Epub 2004/08/25. eng. 
33. Schwartz MA, Ginsberg MH. Networks and crosstalk: integrin signalling spreads. Nature cell biology. 2002 Apr;4(4):E65-8. PubMed PMID: 11944032. Epub 2002/04/11. eng.

34. Geiger B, Spatz JP, Bershadsky AD. Environmental sensing through focal adhesions. Nature reviews Molecular cell biology. 2009 Jan;10(1):21-33. PubMed PMID: 19197329. Epub 2009/02/07. eng.

35. Koo LY, Irvine DJ, Mayes AM, Lauffenburger DA, Griffith LG. Co-regulation of cell adhesion by nanoscale RGD organization and mechanical stimulus. Journal of cell science. 2002 Apr 1;115(Pt 7):1423-33. PubMed PMID: 11896190. Epub 2002/03/16. eng.

36. Huang J, Grater SV, Corbellini F, Rinck S, Bock E, Kemkemer R, et al. Impact of order and disorder in RGD nanopatterns on cell adhesion. Nano letters. 2009 Mar;9(3):1111-6. PubMed PMID: 19206508. Pubmed Central PMCID: PMC2669488. Epub 2009/02/12. eng.

37. Maniotis AJ, Chen CS, Ingber DE. Demonstration of mechanical connections between integrins, cytoskeletal filaments, and nucleoplasm that stabilize nuclear structure. Proceedings of the National Academy of Sciences of the United States of America. 1997 Feb 4;94(3):849-54. PubMed PMID: 9023345. Pubmed Central PMCID: PMC19602. Epub 1997/02/04. eng. 
CHAPTER 7

\section{GENERAL DISCUSSION AND FUTURE OUTLOOK}




\section{Introduction}

The motivation of the work described in this thesis was to identify and study micro- and nanoscale surface topographies with the ability to instruct cell fate and morphology. There is a great potential for innovation of biomaterials, driven by the need to improve their function. Firstly, there is the need for the optimal interaction of medical devices with the host body. For example, the bone integration of artificial hips could be improved to prevent micro motion and extend the implant lifetime. Stents could be improved by reducing protein binding and reducing risk of thrombosis. Secondly, there is the need to improve the function of tissue culture systems. Novel tissue culture systems will increase the predictive value of cell models, increase the yield of protein production systems and help maintain functionality ex vivo to serve in cellbased therapeutics. In vivo cells very rarely if not never encounter very flat surfaces such as the bottom of tissue culture plastics or the smooth surface of implants such as catheters. Yet, while there is a slow increase in demand for alternative tissue culture systems, most life scientists have been culturing cells on flat polystyrene (PS) substrates instead of more advanced materials with nano- and micro-architectures that more closely resemble in vivo situations. One reason for the relative lack of application of surface topography in products is probably that there is no unifying theoretical framework that can explain and predict the underlying mechanisms. To deal with this lack of theoretical framework we used a systematic high-throughput screening (HTS) approach with biomaterials which are similar to the intended (biomedical) application. For this, we used the state of the art Topochip HTS platform which provides a large library of topographies, with great flexibility of substrate biomaterial. This approach provides several topics for discussion in the Chapter: the effect of surface chemistry on the cell topographic interaction, implications of these chemistry effects on the underlying biomolecular mechanisms of cell-topography interactions, the role of cell type on the cell response to topographies, the role of HTS for biomaterials discovery, and upscaling strategies for bioactive topographies.

\section{Surface chemistry as a key player in cell-topography interactions}

The results in both in chapter 4 and in chapter 5 show that the surface chemistry is critical in the process that instructs cell via topography. The osteogenic effect of the topographies identified in chapter 3 was completely absent when these topographies were fabricated in polystyrene instead of titanium (Ti). In fact, the PS topographies inhibited mineralization of human mesenchymal stromal cells (hMSC). Furthermore, PS microtopographies are able to support kidney epithelial cell monolayers while polyether sulfone topographies disrupt the monolayer. This interaction of the effects of surface topography and surface chemistry on cell response makes studying the underlying mechanisms more complex. Looking at literature many different materials are used to study the biological impact of surface topography such as polydimethylsilixane (PDMS) (1), polyurethane (2), polystyrene (PS), (3) polymethyl methacrylate (PMMA) (4) tantalum coated silicon (5), stainless steel (6) and Ti (7). The variation in material choice is very likely dictated by availability of and the experience with existing manufacturing techniques. Most of the model topographies that are used in these investigations require micro-fabrication techniques. These fabrication techniques, usually adapted from the microelectronics field, are often developed for materials that are suitable to process and not because they are relevant in biomedical applications. Furthermore, to overcome the cell culture limitations of materials such as PDMS, ECM coatings such as fibronectin and collagen are often used to increase cell adhesion. Unfortunately, these coatings can obscure topographies and thereby compromise experimental outcomes. Another consequence of choosing inadequate biomaterials for in vitro studies is that they cannot easily be applied in the clinic. The results in this thesis emphasize the importance of biomaterial choice to study the effect the biological effect of surface topography. We made special efforts to conduct our studies in relevant materials which required the 
development of adequate fabrication techniques. The studies conducted in chapter 3 were all conducted on Ti or Ti coated substrates to mimic Ti bone implants. Chapter 5 demonstrated how high-quality microtopographies can be fabricated in porous PolyetherSulphone-Polyvinylpyrrolidone (PES-PVP) membranes to enable application in blood filtration systems. The fabrication of PS topographies was developed for two reasons. Firstly, PS is a practical model material for cell experiments for future studies into the underlying mechanisms of the cell topographies interactions. Secondly, the use of PS TopoChips to identify bioactive topographies readily enables translation into PS tissue culture products. The research in the topography field should be conducted with attention to the eventual application and should be performed with the same biomaterial. This means that that every application requires separate studies with distinct biomaterials. Alternatively it is viable to combine topographies with specialized surface coatings. In this way the bulk material can be something that is compatible with established fabrication methods while the surface chemistry can be tuned to meet future applications. This approach is used in this thesis in chapter 3 with the Ti coating of poly lactic acid. Other coatings could, for example, be calcium phosphate coatings for bone applications (8).

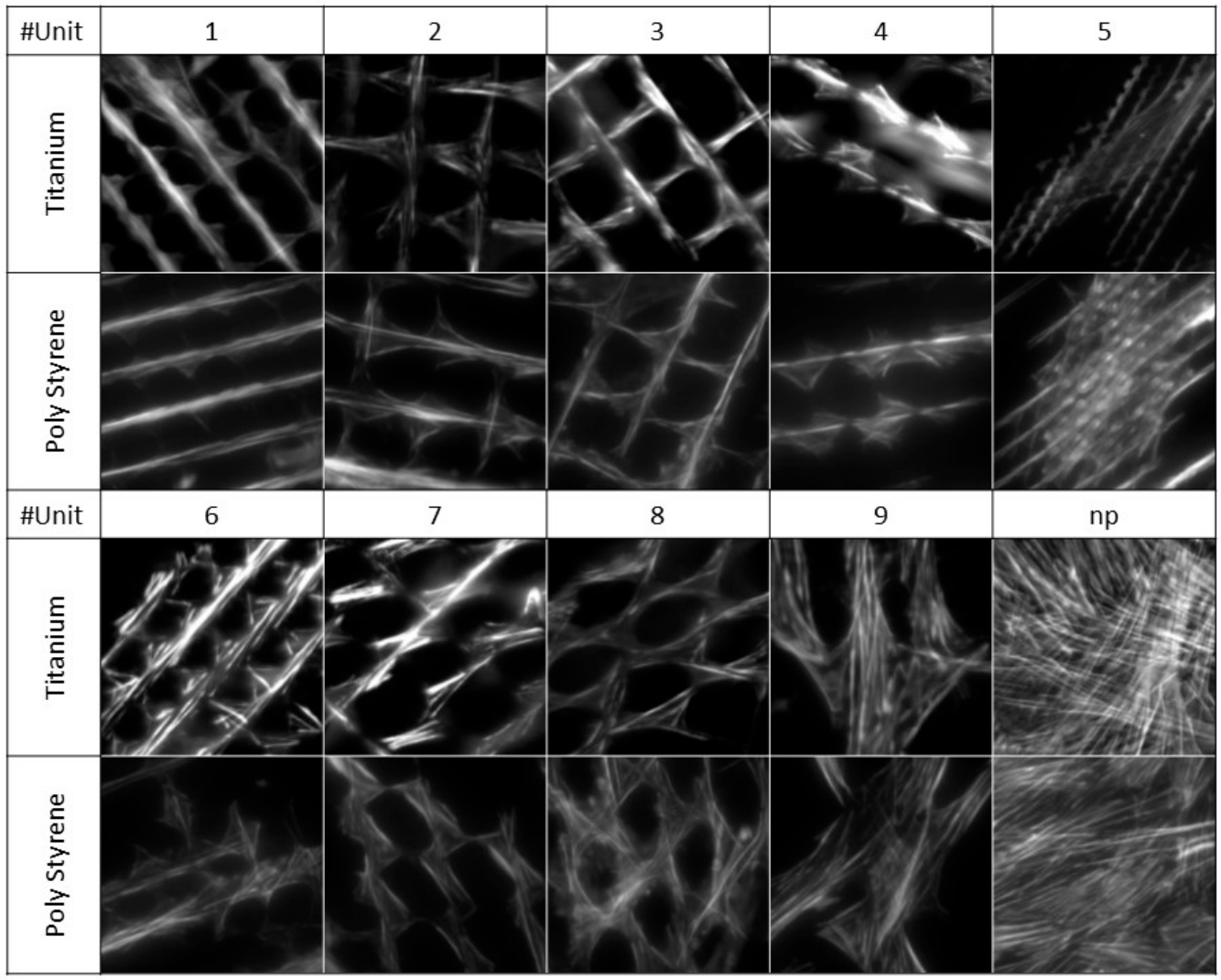

Figure 1: A comparison of cytoskeletal morphologies of hMSCs cultured on PS topographies and titanium topographies. Images from the same topographical feature of materials are paired together. 


\section{Hypotheses on the underlying molecular mechanisms}

In chapter 3 we showed that specific microtopographies are able to induce osteogenic response in vitro and improve bone bonding in vivo. This chapter provided a convincing demonstration of the cell instructive potential of microtopographies, but did not cover the responsible mechanisms. Even though this thesis contains very limited investigation into the underlying molecular mechanisms of the instructive effect of topography we still attempted to provide new hypotheses. The osteogenic topographies induced a cell phenotype that resembled long tube-like cells with thick actin fibers. In an effort to make hypotheses about the underlying mechanisms we speculated on the cellular differences caused by the tube-like morphology between the microstructures, compared to a non-patterned surface. We should also take into account the observation in chapter 4 that showed that this osteogenic affect appears to be material dependent. The same topographies which induced osteogenic response, when fabricated in titanium, inhibited mineralization when fabricated on PS. A visual comparison of the cell morphology induced by the topographies in both materials does not reveal any obvious differences (Fig. 1). This indicates that correlations between cell morphology and cell function are material specific in our case. For nuclear morphology, this phenomena is not in agreement with literature. Thin elongated nuclei (that are also often found on our microtopographies) cause mechanical stresses which are able to enhance Histone $\mathrm{H3}$ acetylation and methylation, independent of the manner and material in which the morphology is induced $(9,10)$. The histone modifications lead to more accessible DNA which increases the plasticity of the cells. Furthermore, increased histone acetylation leads to osteogenic differentiation of hMSCs (11). Nuclear deformation can have effects on chromatin organization (12). In this way cell phenotype could be affected through transcriptional regulation by chromatin remodeling. These mechanisms are very likely to increase the sensitivity to the osteogenic factors but we do not expect these to be the dominant elements in the topography response as elongated nuclei are also very prominent on the PS topographies that did not induce mineralization. To test these hypothesis the effect of histone deacetylase inhibitors and histone acyltransferase inhibitors on the mineralization of hMSCs cultured on titanium topographies could be investigated.

Considering that the induction of the osteogenic phenotype is material specific Integrins may be the key determinant in the bio-mechanical response. Integrins bind cytoskeletal proteins through the cytoplasmic domains and binds extracellular matrix (ECM) proteins with the extracellular domains (13). In this way the Integrins are able not only to act as a physical link between the cytoskeleton and the ECM but also as signaling molecules that enable cells to sense the chemical and mechanical properties of their surroundings. Considering that titanium binds more and perhaps even other proteins due to its high surface energy suggests that there needs to be a certain amount of cell adhesion before the topographies can assert their instructive function (14). This cell adhesion is governed by the amount and potentially even the type of integrin that is able to interact with the binding motifs presented by the proteins on the substrate. This theory is supported by observations that hMSc differentiation is steered by the amount of available RGD (a tripeptide composed of L-arginine, glycine, and L-aspartic acid) motifs and the binding affinity of these motifs. The RGD binding motif interacts with many of the more common integrin types. High amounts of RGD sequences induce osteogenic differentiation while low amount of RGD leads to adipogenic differentiation (15).

Our data in chapter 4 on hMSC adipogenesis supports the role of integrins. Critical for the presence of lipid vesicles in the cells appeared to be the space available between the topographies. The cells are either on top of the topographies when there is limited space between the features or the cells are located between the features when spacing allows it. How the cell 'chooses' between these two states 
probably is based on which of the two is the more energy efficient. Both states lead to very different cell and actin fiber morphologies, very likely associated with the previously described mechanotransduction pathways. Cells which are confined between the topographies appear to produce slightly fewer lipid vesicles. The strongest inhibition of the adipogenic phenotype on PS was observed in cells that bridged the small spaces between the topographies. The gap-bridging could induce a mechanical strain that inhibits adipogenesis. It has been reported that mechanical strain inhibits hMSC adipogenesis by reducing the activity of peroxisome proliferator-activated receptor 2 (PPARy2) (16). How PPARyz activity is regulated by mechanical stress had not been described. There was no formation of lipid vesicles of hMSCs cultured on the titanium substrates. This inhibition of adipogenesis is probably caused by an over-abundance of adhesions sites provided by the surface chemistry of Ti, which is in line with work that shows that limiting integrin binding promotes adipogenesis (15). To investigate the role of integrin amount, clustering and type in the cell response to topography, the microtopographies could be functionalized with various integrin ligands that bind specifically to sub-types of Integrins.

Because of the osteogenic effect of topographies in Chapter 3, another potential molecular factor responsible for the biomechanical response of hMSCs is the Yes kinase associated protein / transcriptional coactivator with PDZ-binding domain (YAP/TAZ) transcription regulating complex (17). Intracellular tension leads to the nuclear translocation of YAP/TAZ and the consequent activation of effector genes promotes the osteogenic response of hMSCs (18). Confinement of the topographies between the topographical features creates mechanical stresses not present on non-patterned substrates. The observation that certain microtopographies are able to limit cell-cell contacts further implicates the role of YAP/TAZ because it is part of the Hippo signaling pathway which is also affected by cell-cell contacts. We have to take into account that in literature, the intracellular tension which causes YAP/TAZ translocation was triggered by differences in substrate stiffness and not microtopography. A difference in stiffness between materials can lead to different cell phenotypes (19). However we do not think that this is a determining factor here, because the stiffness of both materials is relatively similar and neither can be considered as a 'soft' material such as polyacrylamide gels which were used in the before mentioned studies. Unfortunately the role of YAP/TAZ in mechano-transduction has only been established at very early time-points under very specific conditions. Therefore, it is important to consider that the mineralization phenotype occurs at a relatively late time point and at very high cell densities. it could be that the YAP/ TAZ plays in a role in early time point signaling events, which ultimately cascade into a late mineralization if the ECM. It might be interesting to investigate whether the osteogenic effect induces by topography is induced by YAP/TAZ by performing early time point gene expression knockdowns during mineralization on titanium topographies.

\section{The role of cell type in cell-topography interactions}

Although in this thesis no direct comparison between cell types made, several different cell-types were investigated. This allowed us to observe a number of striking differences in response, depending on cell type. In chapter 3 and chapter 4, hMScs were used as a model system to investigate titanium and PS topographies. Kidney epithelial cells were used in chapter 5 and U2OS with stably expressing transgenes were used in chapter 6. A central observation of all the work together is the striking difference between the mesenchymal cells and the epithelial cell types. Due to the fact that the epithelial cells tend to develop into tight clusters on the topographies the impact of the topography was very different compared to mesenchymal cell types. The microtopographies disrupted the epithelial monolayer formation, especially when they were fabricated in PES-PVP. In hindsight is seems logical that the epithelial cells have a superior phenotype on surfaces without large topographical features that have dimensions that are 
in the range of tens of micrometers as they normally interact with a relatively smooth basement membrane in vivo. Epithelial cells require the formation of a tight monolayer with correct junction proteins to maintain their polarity and function (20). The matrix of the basement membrane does have a nanoscale topography. Therefore it could be worthwhile to use a platform such as the NanoTopochip to identify and study nanotopographies which beneficial properties for epithelial cells. Very little literature covers the role of cell type in the instructive effect of topographies. There are some indications that different cell types react to topographical cues in different ways (21).

\section{High-throughput screening of biomaterials}

The design space of new biomaterials is virtually limitless. HTS of biomaterials is a valid approach to investigate many biomaterials or modifications of biomaterials. In this way biomaterials properties can by assayed as well as the biological response. The lack of underlying theoretical framework makes HTS for biomaterials with desired properties very appealing. These experiments may even provide the basis for future hypothesis regarding the underlying mechanisms due the amount of data that can be generated in a short amount of time. Compared to high-throughput screening in pharmaceutical industry, screening biomaterials adds more layers of complexity. The creation of material libraries is relative complex compared to a compound library for example. Most HTS assays rely on imaging as readout, due its speed and low cost. Opaque and/or auto fluorescent materials, such as the PES-PVP used in Chapter 5, often interfere with imaging readouts. For these reasons the HTS of materials has not yet become as common as compound screens or expression profiling. In this thesis we have demonstrated that the Topochip provides as readily accessible platform for HTS. Still, the Topochip is limited by the image-based readout. From the fabrication point of view the TopoChip can be fabricated from most biomedical polymers and metals but the potential auto fluorescence of these materials can make them incompatible with imaging. Another disadvantage is the limited availability and accuracy of existing markers and reporters suitable for imaging. This often leads to qualitative readouts such as the assessment of tight junction formation of kidney epithelial cells in Chapter 5. Because quantitative assays are preferred for screening approaches, robust markers for functional kidney epithelial cells will first have to be developed. First more research has to be conducted to define the molecular characteristics of a functional kidney epithelial cell in vitro before we can start looking biomaterial properties that improve these characteristics.

Moving away from image-based assays could make HTS of biomaterials more powerful. It will likely result in a reduction of throughput but the readouts will provide a lot more information. An example of such a system is the development of the TopoWellplate, which is based on the fabrication technology of the TopoChip. In this system, almost 90 topographies are created on the bottom of standard 96 well plates which can by assayed by techniques such as expression profiling or mass-spectrometry. For example, the L1000 platform (22), which is able to create expression profiles of hundreds or even thousands of samples in tandem should be compatible with the TopoWellplate. Combining HTS technologies such as these will be the future of biomaterials research. Another future prospect is development of more sophisticated screening platforms such as the Nano-TopoChip, shown in chapter 6, made possible by the increased accessibility to state of the art fabrication technologies. In addition to studying cells and tissues, the Nano-Topochip could be a very useful platform to study the effect of surface topography on bio-fouling and bacterial attachment as the topographies have the same size range as many bacteria. Already nanotopographies have been demonstrated to reduce the formation of bacterial biofilms (23). Identifying and applying nanotopographies with anti-bacterial effects could be a viable approach to combat biomaterial associated infections. 


\section{Upscaling of surface topography}

The titanium osteogenic topographies show potential to improve the integration of orthopedic implants. However, so far the topographies have only been tested in rabbits which are only a good model for initial in vivo bone-bonding studies. To improve the evidence for clinical benefit, a logical next step would be to test the osteogenic topographies in a large animal model such as a goat or a sheep which have bone metabolisms that more closely resemble that of humans (24). During these studies it should also be possible to look at long term effects of the designed topographies. Before doing this however it should be decided whether a 'model implant' is used or a 'real implant'. If application of the topographies on a 'model implant' with currently available fabrication technologies can accurately represent a real functional implant this is probably the most economically viable path to take. Alternatively, the topographies have to first be applied to a commonly used bone implant, to be able to test the topographies in a relevant medical intervention. Applying the topographies to an existing bone implant such a dental implant will require the development of new fabrication methods which will require significant investments. One approach is to create topography designs on existing implants by laser-based writing techniques (25) or lithography techniques. Another approach is to use rapid-prototyping techniques such as 3D printing to fabricate the whole implant with the micro-patterns in one step. Although this is currently not yet viable due to lack of resolution, the field of 3D printing of metals for orthopedic implants is advancing rapidly as 3D printed titanium screws have already been implanted (26).

In comparison to up-scaling of topographies for biomedical implants it will be easier to do the up-scaling for cell culture plastics. The production techniques necessary to fabricate PS topographies on large surface areas already exists. For industrial scale production the injection moulding methods will have to be adapted with new moulds which contain the microtopographies. In appendix 2 we conduct a technology assessment to gain more insight into the requirements for the application of microtopographies on tissue culture plastics. In the search for better cell culture substrates it would be interesting to apply topographies to softer materials such as hydrogels. Currently, most of the materials used to study topography are much stiffer than organs and tissues. To mimic the in vivo stiffness with culture substrates it could be interesting to create topographies in hydrogels. For example a Topochip can be made from synthetic hydrogels. In preliminary experiments Topochips have already been made with various hydrogels such as chemically crosslinked polyacrylamide or photo-crosslinked poly D,L lactide/polyethylene glycol macromer networks. Combining microstructures with soft gel systems might be an interesting approach to improve culture substrates for (induced pluripotent) stem cells, as these cells are very sensitive to the culture substrate. Up-scaling such gel systems should be relatively simple. For future applications large stamps could be fabricated and supplied in combination with synthetic hydrogel precursors for in situ topographic gel formation in petri dishes.

\section{Conclusion}

HTS of biomaterials provides many opportunities to study cell-biomaterial interactions such as the cell instructive effect of topography. Not only can bioactive topographies be identified for future applications but the multi-parametric datasets can be used to elucidate relationships between feature dimensions, cell morphology and marker expression. Knowledge of these relationships in combination with information of the surface chemistry can be used to formulate new hypotheses on the underlying molecular mechanisms. To improve the relevance and applicability of bioactive topographies it is critical to use the right biomaterials and cell models. As a biomaterial property topography has great potential for application in medical devices and tissue culture products. 


\section{Literature}

1. Mata A, Kim E), Boehm CA, Fleischman AJ, Muschler GF, Roy S. A three-dimensional scaffold with precise micro-architecture and surface micro-textures. Biomaterials. 2009;30(27):4610-7.

2. Ahn EH, Kim Y, Kshitiz, An SS, Afzal J, Lee S, et al. Spatial control of adult stem cell fate using nanotopographic cues. Biomaterials. 2014 Mar;35(8):2401-10. PubMed PMID: ISI:000331679200001. English.

3. Wang PY, Li WT, YU J, Tsai WB. Modulation of osteogenic, adipogenic and myogenic differentiation of mesenchymal stem cells by submicron grooved topography. J Mater Sci Mater Med. 2012;23(12):3015-28.

4. Dalby MJ, Gadegaard N, Tare R, Andar A, Riehle MO, Herzyk P, et al. The control of human mesenchymal cell differentiation using nanoscale symmetry and disorder. Nature materials. 2007 Dec;6(12):997-1003. PubMed PMID: 17891143. Epub 2007/09/25. eng.

5. Lovmand J, Justesen J, Foss M, Lauridsen RH, Lovmand M, Modin C, et al. The use of combinatorial topographical libraries for the screening of enhanced osteogenic expression and mineralization. Biomaterials. 2009 Apr;30(11):2015-22. PubMed PMID: 19178942. Epub 2009/01/31. eng.

6. De Luca AC, Zink M, Weidt A, Mayr SG, Markaki AE. Effect of microgrooved surface topography on osteoblast maturation and protein adsorption. Journal of biomedical materials research Part A. 2015;28(10):35407.

7. Bornstein MM, Harnisch $\mathrm{H}$, Lussi A, Buser D. Clinical performance of wide-body implants with a sandblasted and acid-etched (SLA) surface: results of a 3-year follow-up study in a referral clinic. The International journal of oral \& maxillofacial implants. 2007 Jul-Aug;22(4):631-8. PubMed PMID: 17929525. Epub 2007/10/13. eng.

8. Narayanan R, Seshadri SK, Kwon TY, Kim KH. Calcium phosphate-based coatings on titanium and its alloys. Journal of biomedical materials research Part B, Applied biomaterials. 2008 Apr;85(1):279-99. PubMed PMID: 17853421. Epub 2007/09/14. eng.

9. Li Y, Chu JS, Kurpinski K, Li X, Bautista DM, Yang L, et al. Biophysical regulation of histone acetylation in mesenchymal stem cells. Biophysical journal. 2011 Apr 20;100(8):1902-9. PubMed PMID: 21504726. Pubmed Central PMCID: PMC3077706. Epub 2011/04/21. eng.

10. Downing TL, Soto J, Morez C, Houssin T, Fritz A, Yuan F, et al. Biophysical regulation of epigenetic state and cell reprogramming. Nature materials. 2013 Dec;12(12):1154-62. PubMed PMID: 24141451. Epub 2013/10/22. eng.

11. de Boer J, Licht R, Bongers M, van der Klundert T, Arends R, van Blitterswijk C. Inhibition of histone acetylation as a tool in bone tissue engineering. Tissue engineering. 2006 Oct;12(10):2927-37. PubMed PMID: 17518660. Epub 2007/05/24. eng.

12. Makhija E, Jokhun DS, Shivashankar GV. Nuclear deformability and telomere dynamics are regulated by cell geometric constraints. Proceedings of the National Academy of Sciences of the United States of America. 2016 Jan 5;113(1):E32-40. PubMed PMID: 26699462. Epub 2015/12/25. eng.

13. Humphries JD, Byron A, Humphries MJ. Integrin ligands at a glance. Journal of cell science. 2006 Oct 1;119 (Pt 19):3901-3. PubMed PMID: 16988024. Pubmed Central PMCID: PMC3380273. Epub 2006/09/22. eng.

14. Zhao G, Schwartz Z, Wieland M, Rupp F, Geis-Gerstorfer J, Cochran DL, et al. High surface energy enhances cell response to titanium substrate microstructure. Journal of biomedical materials research Part A. 2005 Jul 1;74(1):49-58. PubMed PMID: 15924300. Epub 2005/06/01. eng.

15. Kilian KA, Mrksich M. Directing stem cell fate by controlling the affinity and density of ligand-receptor interactions at the biomaterials interface. Angewandte Chemie (International ed in English). 2012 May 14;51 (20):4891-5. PubMed PMID: 22505230. Pubmed Central PMCID: PMC3754806. Epub 2012/04/17. eng.

16. Case N, Thomas J, Xie Z, Sen B, Styner M, Rowe D, et al. Mechanical input restrains PPARgamma2 expression and action to preserve mesenchymal stem cell multipotentiality. Bone. 2013 Jan;52(1):454-64. PubMed PMID: 22951341. Pubmed Central PMCID: PMC3535265. Epub 2012/09/07. eng.

17. Aragona M, Panciera T, Manfrin A, Giulitti S, Michielin F, Elvassore N, et al. A mechanical checkpoint controls multicellular growth through YAP/TAZ regulation by actin-processing factors. Cell. 2013;154(5):1047-59. 
18. Dupont $\mathrm{S}$, Morsut L, Aragona M, Enzo E, Giulitti S, Cordenonsi M, et al. Role of YAP/TAZ in mechanotransduction. Nature. 2011;474(7350):179-83.

19. Engler AJ, Sen S, Sweeney HL, Discher DE. Matrix elasticity directs stem cell lineage specification. Cell. 2006 Aug 25;126(4):677-89. PubMed PMID: 16923388. Epub 2006/08/23. eng.

20. Giepmans BN, van ljzendoorn SC. Epithelial cell-cell junctions and plasma membrane domains. Biochimica et biophysica acta. 2009 Apr;1788(4):820-31. PubMed PMID: 18706883. Epub 2008/08/19. eng.

21. Biela SA, Su Y, Spatz JP, Kemkemer R. Different sensitivity of human endothelial cells, smooth muscle cells and fibroblasts to topography in the nano-micro range. Acta biomaterialia. 2009 Sep;5(7):2460-6. PubMed PMID: 19410529. Epub 2009/05/05. eng.

22. Peck D, Crawford ED, Ross KN, Stegmaier K, Golub TR, Lamb J. A method for high-throughput gene expression signature analysis. Genome biology. 2006;7(7):R61. PubMed PMID: 16859521. Pubmed Central PMCID: PMC1779561. Epub 2006/07/25. eng.

23. Feng G, Cheng Y, Wang SY, Hsu LC, Feliz Y, Borca-Tasciuc DA, et al. Alumina surfaces with nanoscale topography reduce attachment and biofilm formation by Escherichia coli and Listeria spp. Biofouling. 2014;30 (10):1253-68. PubMed PMID: 25427545. Epub 2014/11/28. eng.

24. von Rechenberg B. Animal models in bone repair. Drug Discovery Today: Disease Models. 2014/Autumn;13:23-7.

25. Ertorer E, Vasefi F, Keshwah J, Najiminaini M, Halfpap C, Langbein U, et al. Large area periodic, systematically changing, multishape nanostructures by laser interference lithography and cell response to these topographies. Journal of biomedical optics. 2013 Mar;18(3):035002. PubMed PMID: 23460125. Epub 2013/03/06. eng.

26. Yang J, Cai H, LV J, Zhang K, Leng H, Wang $Z$, et al. Biomechanical and histological evaluation of roughened surface titanium screws fabricated by electron beam melting. PloS one. 2014;9(4):e96179. PubMed PMID: 24788866. Pubmed Central PMCID: PMC4005762. Epub 2014/05/03. eng. 
APPENDIX 1

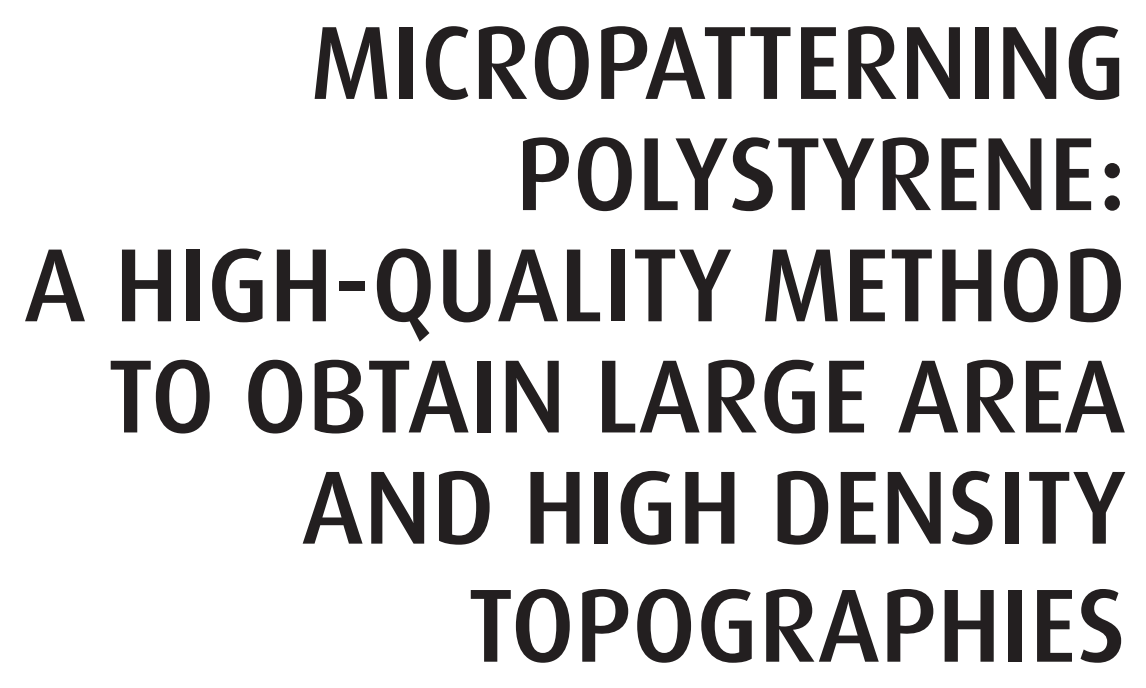

Developed by Yiping Zhao. 


\section{Materials and fabrication Process scheme}

Before starting the PS microfabrication process, the silicon master mould, which contains the inverse pattern of the topographies, is fabricated by silicon micromachining technologies as is described in Chapter 3. The The silicon mould is first cleaned in Piranha solution $\left(\mathrm{H}_{2} \mathrm{SO}_{4}: \mathrm{H}_{2} \mathrm{O}_{2}=3: 1 \mathrm{~V} / \mathrm{V}\right.$, at a temperature of about $100^{\circ} \mathrm{C}$ ) for $30 \mathrm{~min}$, rinsed with deionized (DI) water, spun dry with $\mathrm{N}_{2}$ and finally a monolayer of trichloro(1H, 1H, 2H, 2H-perfluorooctyl)silane (FOTS, Sigma-Aldrich) is deposited on the mould from gas phase under vacuum conditions in a desiccator. Afterwards, the fabrication of intermediate PDMS and OrmoStamp ${ }^{\circledR}$ moulds and PS hot embossing follows the scheme as illustrated in Figure 1. The complete process scheme consists of phases: (1) PDMS mould fabrication; (2) Ormostamp ${ }^{\circledR}$ mould fabrication; (3) PS hot embossing.

\section{PDMS mould fabrication}

The curing agent and the base (Sylgard $184^{\circledR}$ silicone elastomer kit, Dow Corning Corporation) are well mixed (1:10, w/w) and degassed in a desiccator. $12 \mathrm{~mL}$ PDMS pre-polymer is cast on a full 100 $\mathrm{mm}$ silicon wafer to receive a 1-1.5 mm thick PDMS mould. After casting the prepolymer on the silicon mould (Fig. 1b), it is cured on a leveled hot plate or oven at $80^{\circ} \mathrm{C}$ for a minimum of $8 \mathrm{~h}$ (Fig. 1C). The PDMS film is peeled off from the silicon mould (Fig. 1d) and can be readily used to fabricate Ormostamp ${ }^{\circledR}$ mould.

\section{OrmoStamp ${ }^{\circledR}$ mould fabrication}

OrmoStamp ${ }^{\circledR}$ and OrmoPrime ${ }^{\circledR} 08$ are bought from Micro Resist Technology GmbH and can readily be used. OrmoStamp ${ }^{\circledR}$ is an UV curable inorganic-organic hybrid polymer and OrmoPrime ${ }^{\circledR} 08$ is the adhesion promoter for Ormostamp ${ }^{\circledR}$. Detailed information about these two polymers can be found in the manufacturer's processing guidelines. The fabrication process starts with Borofloat wafers (Borofloat ${ }^{\circledR} 33$ from Schott) of $100 \mathrm{~mm}$ diameter and $500 \mu \mathrm{m}$ thickness (Fig. 1e). To prepare the OrmoStamp ${ }^{\circledR}$ mould for hot embossing, a gentle $\mathrm{O}_{2}$ plasma treatment is performed using reactive ion etching (RIE, home-build) at 10 ${ }^{\circ} \mathrm{C}$, $50 \mathrm{sccm} \mathrm{O}_{2}$ flow, 75 mTorr pressure, and 50W CCP power for $30 \mathrm{~s}$. A monolayer of FOTs is deposited from gas phase under vacuum condition in a desiccator).

\section{Polystyrene film preparation and hot embossing}

Biaxially oriented PS films of $190 \mu \mathrm{m}$ were bought from Goodfellow, United Kingdom and readily used. The PS hot embossing process is performed using the Obducat Eitre ${ }^{\circledR} 6$ Nano Imprint Lithography system (Obducat, Sweden). To prepare the OrmoStamp ${ }^{\circledR}$ mould (Fig. 1j) contains the pattern that is transferred to the PS film. The PS film and OrmoStamp ${ }^{\circledR}$ mould are brought into contact (Fig. 1k) and the hot embossing is performed at a temperature of $140^{\circ} \mathrm{C}$ and a pressure of 10 bar for 5 min (Fig. 1l). Finally, the imprinted PS film is separated from the OrmoStamp ${ }^{\circledR}$ mould at $90^{\circ} \mathrm{C}$ (Fig. 1m). 
PDMS mould fabrication

a

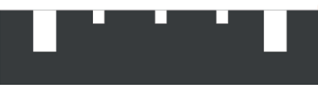

b

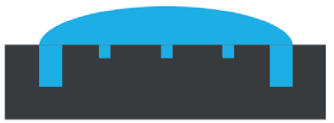

C

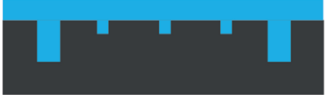

d
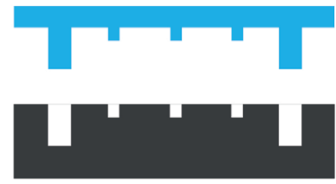

OrmoStamp ${ }^{\circledR}$ mould fabrication

e
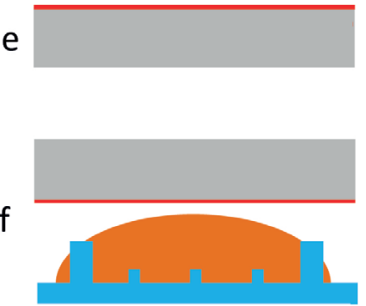

k

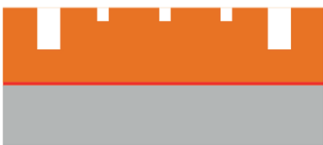

g
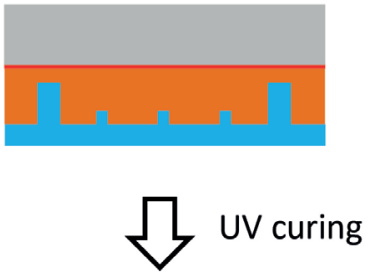

h
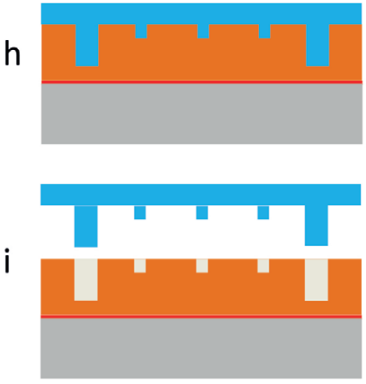

m
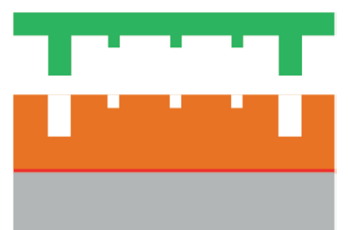
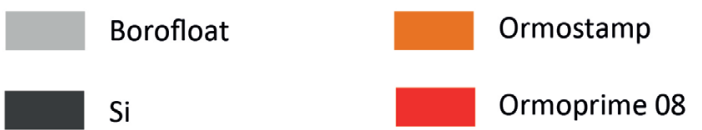

PDMS

PS

\section{Figure 1. Polystyrene topography microfabrication scheme.}

After Piranha cleaning (procedure as described in section 1) and dehydration bake on a hot plate at $120^{\circ} \mathrm{C}$ for a minimum of 10 min, OrmoPrime 08 is spin-coated on the Borofloat wafer at $4000 \mathrm{rpm}$ for $30 \mathrm{~s}$ and then baked on a hot plate at $150^{\circ} \mathrm{C}$ for $5 \mathrm{~min}$. In our experiment, Borofloat wafer with OrmoPrime ${ }^{\circledR} 08$ is prepared right before the application of OrmoStamp ${ }^{\circledR}$. A droplet of $1.5 \mathrm{~mL}$ Ormostamp ${ }^{\circledR}$ is slowly dispensed on the PDMS mould (Fig. 1f) and is slowly brought into contact with the Borofloat wafer with OrmoPrime ${ }^{\otimes} 08$ coating. Slow spreading of the droplet between the Borofloat wafer and PDMS mould avoids air bubbles getting trapped. Filling of the gap between the two substrates is completed by capillary force and takes about 15-30 min (Fig. 1g). The PDMS and Borofloat wafer stack is exposed to 350-450 nm UV light for $90 \mathrm{~s}$ with the light intensity set at $12 \mathrm{~W} / \mathrm{cm}^{2}$ (EVG $620 \mathrm{i}$-line exposure system) (Fig. 1h). The PDMS mould can easily be peeled off from the OrmoStamp ${ }^{\circledR}$ mould, after which the Ormostamp ${ }^{\circledR}$ mould immediately follows a hard bake process at $130^{\circ} \mathrm{C}$ on a hot plate (ramping up from $20^{\circ} \mathrm{C}$ to $130^{\circ} \mathrm{C}$ with a ramping speed of $5^{\circ} \mathrm{C} / \mathrm{min}$ ). After hard baking for $30 \mathrm{~min}$ at $130^{\circ} \mathrm{C}$, the Ormostamp ${ }^{\circledR}$ mould is cooled down together with the hot plate to room temperature

(Fig. 1i). The complete hard bake takes about $3 \mathrm{~h}$. 


\section{APPENDIX 2}

\section{PLURIPOTENCY INDUCING TOPOGRAPHY FOR TISSUE CULTURE: A TECHNOLOGY ASSESSMENT ON THE TRANSLATION OF DESIGNED TOPOGRAPHY TOWARDS A CELL-CULTURE PRODUCT}

Frits Hulshof and Verena Stimberg 
We have used high-throughput screening to identify several surface topography designs with significant biological effect which could be interesting for cell culture plastics or to structure the surface of medical implants. One question is if this technology can be applied to create a product that can fulfill a market demand. Topographies will most likely sooner be applied to tissue culture products than to medical implants because of two main reasons. Firstly, it is much cheaper to prove in vitro efficacy required of a tissue culture product than to prove clinical efficacy required of a medical implant. Large scale clinical trials usually cost many millions and can take many years to determine the long term effects. Secondly, the fabrication methods that are used to create the topographical libraries are much closer technologically to the production methods of tissue culture plastics than medical implants. Applying a surface modification to a device with relatively complex dimensions such as an artificial hip is a lot more complex than applying a surface modification to a two dimensional tissue culture plate. For these reasons we want to investigate the possibility and the potential to commercialize the topographies into a tissue culture product in the form of a brief technology assessment by making use of a socio-technical map.

Conventional flat culture plastics have been the standard for decades even though one could argue that they have extreme biophysical effects on cells, compared to an in vivo situation (1). Providing cells with microstructures that allow cell attachment in more than one dimension creates a different biophysical environment. In this thesis we have presented multiple examples of the strong effects of surface microtopography on various cell types. The biomechanical effects of surface topography are cell-specific. If we aim to sell a tissue culture product with topographies it should work on a cell type that is widely used. For this reason we propose specialized tissue culture plastics for the widely used induced pluripotent stem cells (iPSC) as a case for our technology assessment. The socio-technical maps of state of the art tissue culture plastics and the potential tissue culture product with bioactive topography that were created during a technology assessment exercise are shown in Figure 1. The socio-technical map shows the technology, regulation, user practices, cultural meaning and infrastructure of a socio-technical system (2) and serves as an overview of the elements of a technology that are required for a product and its societal embedding. The two socio-technical maps are used to compare existing technology, in this case the standard tissue culture plastics, with the map of a new technology (topographic culture plastics). By comparing these maps you can look at the differences which give indications about possible obstacles that need to be addressed in order to introduce such a product (red text), the improvements that such a new technology would induce (green text), and the aspects where little change is anticipated (black text).

Studies with our topography libraries have identified topographical designs that are able to maintain pluripotency proliferation of iPSC maintenance in vitro (3). The topographies serve as a xeno-free alternative to the ECM proteins. Typically, the survival, proliferation and pluripotency of iPSC relies on cell attachment to ECM components, which are usually derived from animal sources. For clinical application of iPSC it is necessary to find alternatives to ECM proteins to work with xeno-free products and to increase reproducibility. Culture of iPSC without the need for coatings is assumed to be the main selling point of the microstructured tissue culture product. iPSC are expected to be the future of many stem cell based therapies.

This is because iPSC can be generated from the patients owns cells. The idea is that these patient specific iPSC can be both used to model the disease to identify and characterize successful therapeutics and also as a therapeutic agent themselves. Many neurological, hematologic and metabolic diseases that are currently untreatable are prime candidates for iPSC based therapies. Efficient iPSC xeno-free production will be very important for the development and production of these therapies. For this reason we believe there is a current and certainly a future market for specialized iPSC tissue culture products both in life science and pharmaceutical industry. 


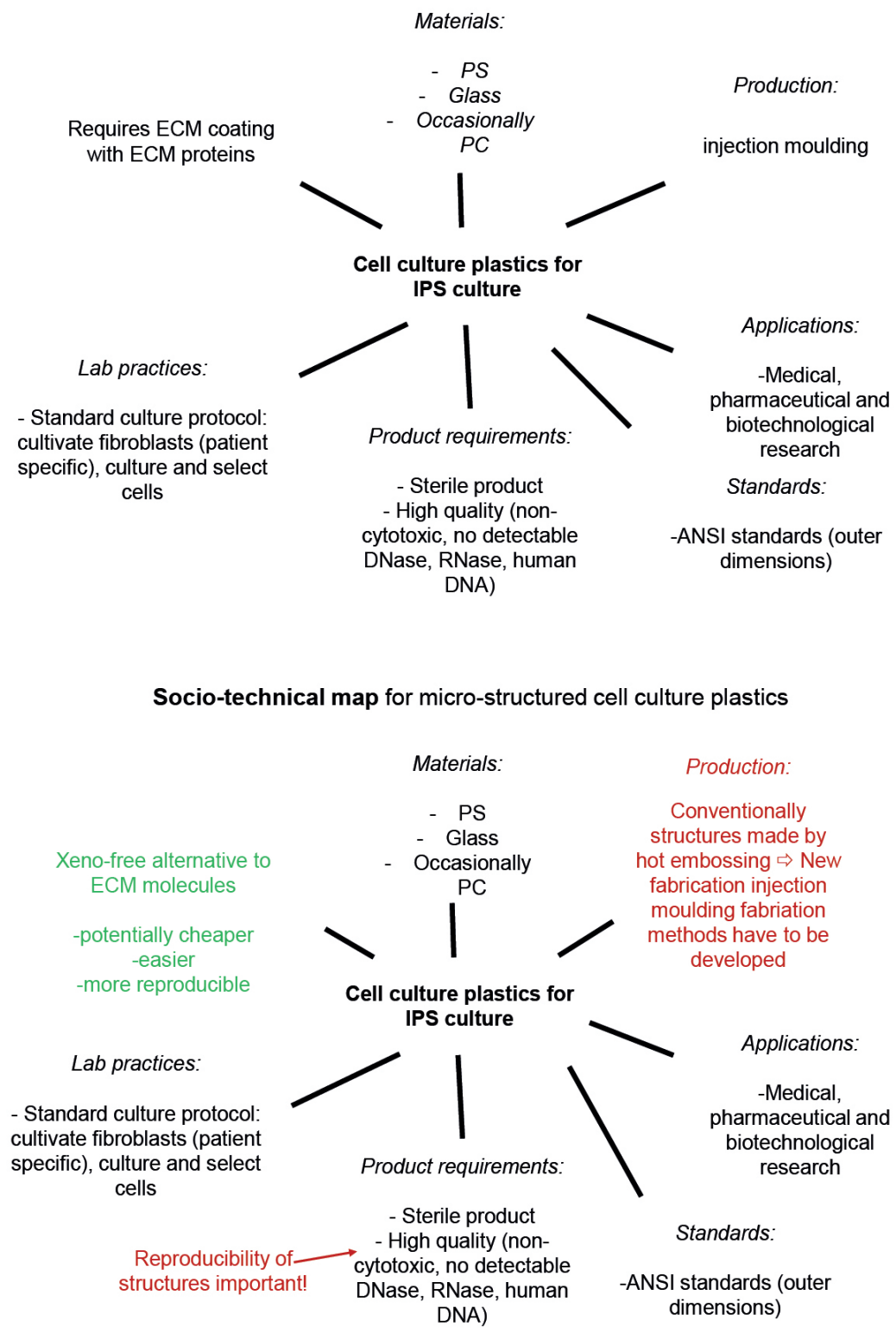

Figure 1: Socio-technical maps of tissue culture plastics (top) and tissue culture plastics modified with microtopographies (bottom). Red text indicates possible obstacles that need to be addressed in order to introduce such a product. Green text indicates the improvements that such a new technology would induce. Aspects were little change is expected are shown in black. 
As displayed in the socio-technical map, most of the current tissue culture products are produced from high molecular weight polystyrene (PS). The experiments that were performed to identify the iPSC maintenance topography designs used topographical libraries produced in PS. The aim is to produce the topographies in the same PS that is currently being used for tissue culture products. Because the material stays the same we anticipate that the product will be more interesting for potential customers who might not want to change from their current material.

Concerning the production method it is a necessary step to translate the fabrication method of the topographies, which uses hot embossing, to an injection-moulding method which is currently used to produce culture plastics and which is suitable for large scale production. The moulds could perhaps be made in stainless steel with laser cutting. This probably means a large initial cost of mould development and fabrication, and adaptation to current state of the art production processes in tissue culture plastic industry. For these reasons we expect the resulting product to have a higher production cost of conventional plastic ware. However, the potential to culture without the need of ECM products will probably make customers willing to pay a higher price because they will save on ECM products.

We expect that the topographical designs can be implemented within the ANSI standards of the existing cell culture dish formats such as 6, 12, 24, 28 and 96 well plates as well as flasks. Within our group we already have a working 'TopoWell' prototype of a 96 well-plate which contains a relatively small library of topographies, compared to the Topochip but provided a much larger surface area, suitable for RNA isolation or proteomics studies. The TopoWell plate is created by hot embossing and has a very good reproducibility of the microstructures, which is also deemed necessary for culture plastics fabrication.

The socio-technical map was created by us, Frits Hulshof and Verena Stimberg, based on public sources of information and discussions. The next step is to take into account the perspective from a party that is more involved in the actual process of commercially producing/selling such a product. When we approached tissue culture manufacturers with the question to give some feedback to our technology assessment exercise we received various replies. Some companies indicated that providing feedback to our plan would reveal too much sensitive information which might indicate that they are in the process of developing a similar product (direct quote from a company reply: "The topic of structured cell culture surfaces is to closely connected to our interests. We would disclose too much of our plans, if we answer the questionnaire."). An R\&D researcher from another company explained to us that although in general our idea is good he foresees that the production of moulds for injection moulding would be too expensive. He was afraid that the small size of the current market would not justify these costs. Yet, due to the relatively large scale of the topographies (larger than $10 \mu \mathrm{m}$ ) the costs might be lower than expected. He suggested that it would perhaps be more viable to use the existing hot embossing method to produce films with topographies which can be used as inlay for conventional products so that the small-scale production rate might be adequate for a small market.

We think that one important step to convince industry to use our topographies in a novel tissue culture product is to show that the topographical designs work when they are up-scaled to larger surface areas and that they perform their function over longer periods of time. On the other hand, customers of the cell culture community are not easily willing to accept new, even improved, cell culture products but rather stick to established products. Here, a more in-depth technology assessment is required to confirm who would potentially buy the product and what their specific demands are. In a next step, various stakeholders such as technology developers, manufacturers and potential customers can be brought together to learn about each other's expectations and to take that into account for future developments.

In summary, we expect that the new products will fit in the established working procedures (in terms of materials, dimensions, compatibility with working procedures/chemicals etc.), and that they fulfill the standards and regulations. On the other hand, fabrication processes must be adapted. Therefore, it has to be determined if the investment in the adaptation of excisting production methods can be earned back by higher product prizes that can be demanded for the ability to culture in xeno-free conditions. Possibly 
the potential market of iPSC users first has to grow before a large investment in production technology is warranted. Additionally, customer demands must be taken into account to ensure acceptance of the new product.

\section{Literature}

1. Gasiorowski JZ, Murphy CJ, Nealey PF. Biophysical cues and cell behavior: the big impact of little things. Annual review of biomedical engineering. 2013;15:155-76. PubMed PMID: 23862676. Epub 2013/07/19. eng.

2. Geels FW. The dynamics of transitions in socio-technical systems: A multi-level analysis of the transition pathway from horse-drawn carriages to automobiles (1860-1930). Technology Analysis \& Strategic Management. 2005 2005/12/01;17(4):445-76.

3. Reimer A, Vasilevich A, Hulshof F, Viswanathan P, van Blitterswijk CA, de Boer J, et al. Scalable topographies to support proliferation and Oct4 expression by human induced pluripotent stem cells. Scientific reports. 2016;6:18948. PubMed PMID: 26757610. Epub 2016/01/14. eng. 


\section{Summary}

The control of biomaterial surface topography is emerging as a tool to influence cells and tissues. Due to a lack a theoretical framework of the underlying molecular mechanisms high-throughput screening (HTS) technology is valuable to identify and study bioactive surface topographies. In Chapter $\mathbf{1}$ the methods and techniques required for HTS of topographies, such as the Topochip topographical library are briefly explained. The Topochip, which is employed throughout this thesis, relies heavily on high-content imaging techniques to study the biomaterials-cell interactions. Chapter 2 provides a clear tutorial and highlights the many possibilities, advantages and disadvantages that come with high-throughput, high-content imaging.

Allready, surface topography is applied to titanium orthopedic implants to improve clinical outcome. Chapter 3 demonstrates how the titanium coated TopoChip is used to identify osteogenic topographies that are able to enhance osteogenic differentiation of human mesenchymal stromal cells (hMSc) in vitro and are able to improve titanium bone implant performance in vivo. Furthermore, the chapter describes how the screening system allows identification of instructive topographies and also provides the opportunity to control design parameters and monitor the effects which allows the inference of relationships between topographical feature shapes, cell morphology and cell differentiation, analogous to identification of drug identification by computational chemistry. In Chapter 4 we studied the instructive effect of Polystyrene (PS) on differentiation of hMSCs. we showed that the microtopographies are able to strongly inhibit osteogenesis by lowering ALP expression and mineralization, without affecting cell density. Additionally adipogenesis is inhibited by several microtopography designs, depending on spacing of the features. Finally, when comparing the differentiation of hMSC cultured on PS topographies to hMSCs cultured on the exact same Ti topographies the contrasting outcomes demonstrate the importance of substrate material choice in these studies.

Chapter $\mathbf{5}$ describes the effects of membrane chemistry and surface topography on kidney epithelial cells in order to improve functional monolayer formation. For this we fabricated designed microtopographies with high resolution and reproducibility on PS films on PolyetherSulphone-Polyvinylpyrrolidone (PES-PVP) porous membranes. A conditionally immortalized proximal tubule epithelial cell line (ciPTEC) was cultured on both the PS topographies and the PES-PVP topographical membranes. Our results showed that L-dopamine coating of the PES-PVP was sufficient to support ciPTEC monolayer formation. The designed microtopographies of both materials affected ciPTEC morphology in different ways. Whereas the PS topographies with large features were able to align the cells in various patterns without significantly disrupting monolayer formation, the PES-PVP topographies with large features easily disrupted the monolayer. PES-PVP topographies with small features and with large spacing supported the ciPTEC monolayer formation well.

Chapter 6 outlines the development of the Nano-TopoChip which has topographical features of lateral dimensions between $200 \mathrm{~nm}$ and $2 \mu \mathrm{m}$, which is more than 50 times smaller than the micro-Topochip. For the fabrication of nano-sized features on a large area of $4 \mathrm{~cm}^{2}$ the use of deep UV lithography, normally only used in chip industry, was required. The cell morphological effect of the nanotopographies was quantified with actin-RFP labeled U2OS osteosarcoma cells that were cultured and imaged on the Nano-Topochip. First, a comparison between U20S cultured on the micro-TopoChip and the Nano-TopoChip indicated that the nanoscale topographies mainly affect actin fiber morphology without the cell shape guidance which occurs on the microtopographies. Subsequent automated image analysis showed that of many cell morphological parameters, cell spreading, cell orientation and actin morphology were most affected by the nanotopographies. Additionally, by using modelling, the changes of cell morphological parameters could by predicted by several feature shape parameters such as lateral size and spacing. 
Chapter 7 presents general discussion of the thesis findings and an outlook of the work. A technology assessment on concept of applying micro topographies to tissue culture plastics is performed in Appendix 2. 


\section{Samenvatting}

De controle over de oppervlakte topografie van biomaterialen is een opkomende manier om cellen en weefsels te sturen. Omdat alomvattende, bruikbare kennis over de onderliggende biomoleculaire mechanismen nog ontbreekt, is 'high-throughput screening' (HTS) een waardevolle manier om bioactieve oppervlakte topografieën te identificeren en te bestuderen. HTS is een aanpak waarbij er in één experiment zeer veel, meestal meer dan enkele duizenden, metingen worden gedaan, waardoor er een zeer grote dataset gegenereerd wordt. In hoofdstuk 1 worden de methoden en technieken die nodig zijn voor HTS van biomateriaal eigenschappen zoals de Topochip beschreven. De Topochip technologie die veel gebruikt wordt in deze thesis is sterk afhankelijk van microscopische technieken waarbij er zeer veel metingen worden aan grote sets van afbeeldingen, ook wel 'high-content imaging' genoemd. Hoofdstuk 2 geeft een introductie over high-content imaging warbij de vele mogelijkheden, voordelen en nadelen worden belicht.

Oppervlakte topografie wordt al in de kliniek toegepast om de functie van orthopedische bot implantaten te verbeteren. Hoofdstuk 3 laat zien hoe titanium gecoate Topochips worden gebruikt om topografieën te ontdekken die de osteogene differentiatie van humane mesenchymale stromale cellen (hMSC) bevorderen. Deze osteogene topografieën blijken bovendien de botvorming in een proefdiermodel te verbeteren. Verder laten we zien hoe de grote dataset, die verkregen is met de systematische screening techniek, gebruikt kan worden om modellen te maken die de relaties laten zien tussen de ontwerp parameters van de topografieën, de cel morfologie en de differentiatie van deze cellen. In hoofdstuk 4 staat hoe we het cel sturende effect van topografieën, gemaakt in polystyreen (PS), op de differentiatie van hMSc hebben bestudeerd. We laten zien dat de topografieën in staat zijn om de osteogene differentiatie van hMSC sterk te remmen door de expressie van Alkaline fosfatase te verlagen en de mineralisatie te verminderen, zonder de celdichtheid sterk te beïnvloeden. Ook de adipogene differentiatie van hMSC werd geremd door te topografieën, afhankelijk van de dimensies en ruimte tussen de structuren. Ten slotte bleek dat de PS topografieën het tegenovergestelde effect hadden op hMSC als titanium topografieën wanneer deze direct vergeleken werden. Hieruit kunnen we concluderen dat het materiaal waarin in de topografie gemaakt is een cruciale factor is bij de manier waarop de topografieën de cellen beïnvloeden.

Hoofdstuk 5 beschrijft de effecten van membraan chemie en oppervlakte topografie op nier epitheel cellen met het doel om de vorming van functionele cel monolagen te verbeteren. Hiervoor hebben we specifieke microtopografieën gemaakt op PS films en Polyethersulfone-Polyvinylpyrrolidone (PES-PVP) poreuze membranen. Een conditioneel onsterfelijk gemaakte proximale tubulus epitheel cellijn (ciPTEC) was gekweekt op zowel de PS films als de PES-PVP membranen. De resultaten lieten zien dat een L-Dopamine coating nodig was voor de kweek van ciPTEC op de PES-PVP membranen. De topografieën, gemaakt in de beide materialen beïnvloedden de ciPTEC morfologie op verschillende manieren. Terwijl de PS topografieën met grote structuren in staat waren om de cellen richting één lijn te oriënteren zonder daarbij de monolaag te verstoren, verstoorden de PES-PVP topografieën de monolaag wel. PES-PVP topografieën met kleine structuren en veel ruimte tussen de structuren waren wel in staat om ciPTEC monolagen mogelijk te maken.

Hoofstuk 6 omschrijft de ontwikkeling van de Nano-TopoChip die topografieën heeft met laterale dimensies die variëren tussen de $200 \mathrm{~nm}$ en $2 \mu \mathrm{m}$, wat meer dan 50 keer kleiner is dan de (micro) Topochip. Om zulke kleine structuren te kunnen maken op een relatief groot oppervlak van $4 \mathrm{~cm}^{2}$ was het nodig om diep UV lithografie te gebruiken, een techniek die normaal gesproken alleen wordt toegepast in de chipindustrie. Het effect van de nanotopografieën op cel morfologie werd gemeten door actine-RFP gelabelde U2OS cellen te kweken en microscopisch te fotograferen op zowel de micro Topochip als de Nano-Topochip. Eerst hebben we UzOS morfologie vergeleken tussen de twee verschillende TopoChips. Het bleek dat de 
nanotopografieën vooral de actine fiber morfologie beïnvloedde in tegenstelling tot de microtopografieën die vooral de totale vorm van de cellen beïnvloedde. De geautomatiseerde fotoanalyse liet zien dat van de vele cel morfologische parameters die er gemeten waren, vooral de cel spreiding, cel oriëntatie en actine morfologie beïnvloed werden door de nanotopografieën. Daarnaast bleken we in staat om met behulp van modellen de cel morfologische parameters te voorspellen aan de hand te ontwerpparameters van de nanostructuren zoals laterale grootte en de ruimte tussen de structuren.

Hoofdstuk 7 bestaat uit een algemene discussie van de bevindingen in deze thesis en ook een vooruitblik op toekomstig werk. Een zogenaamde 'Technology Assessment' over het idee om microschaal topografieën toe te passen in kweekplastics wordt uitgevoerd in Appendix 2. 


\section{Dankwoord - Acknowledgements}

Een promotie gaat een stuk sneller dan je denkt en toch zijn er in die tijd een groot aantal mensen belangrijk geweest in deze tijd. In dit laatste hoofdstuk zal ik proberen iedereen te hiervoor bedanken.

Ten eerste wil ik beginnen met het bedanken van mijn twee promotoren Jan de Boer en Dimitrios Stamatialis. Jan, je hebt me veel geholpen met het samen brainstormen naar creatieve oplossingen voor de vele obstakels die je tegenkomt tijden een promotieonderzoek. Hoewel het soms lastig bleek om tijd te kunnen plannen door je drukke schema, sabbatical naar Boston en de verhuizing naar Maastricht denk ik dat onze gesprekken altijd heel productief zijn geweest. In tijden dat ik toch wat pessimistisch was over enkele aspecten van mijn onderzoek was het jouw optimisme dat me toch weer een duwtje in de rug gaf. Vaak moest je me vertellen om blij en trots te zijn op resultaten waarvan ik zelf vond dat het 'wel aardig' was omdat het toch in mijn aard ligt om te kijken naar alles wat er nog moet gebeuren in plaats van te beseffen wat er allemaal al bereikt is. Dimitrios, you helped me a lot with the process of turning all the results in appealing stories. My dry factual writing style needed constant corrections. You would often help me when I was stuck with a piece of text of which I could not make sense of the storyline. You are one of the most professional people I know and I have a lot of respect for the way in which you combine your work with your family. Especially so during the times were things are difficult due to health problems in your family. I hope they are doing better now and will continue to do so. Clemens van Blitterswijk wil ik hier bedanken voor deelname aan mijn promotiecommissie. Het was door een gesprek met jou na het zien van jouw presentatie dat ik contact heb opgenomen met jan over de mogelijkheid om een promotie te gaan doen.

De Vakgroep BST heeft een grote rol gespeeld tijden mijn promotie. Mede door de goede sfeer hier ging ik elke dag met plezier naar de Universiteit. Dirk, je zorgde ervoor dat ik me thuis voelde in jouw vakgroep, bedankt daarvoor. Karin, je hebt me veel geholpen met de hele papierwinkel. Je bent een onmisbare kracht die zorgt dat alles altijd goed geregeld is. Ook bedank ik Aga, Shariar, Marlon, Seb, Suvi, Mark, Denys, Natalia, Odyl, Thijs, Jos, Andre, Aysun, Ilaria, Kasia, Marlon, Tony, Gijs, Vincent, Lydia, Hetty, Anita, Marc en alle masterstudenten voor de gezelligheid tijdens pauzes, borrels en andere activiteiten.

De vakgroep TR die later verhuisde naar Maastricht en daar het MERLN instituut heeft gevormd was de andere belangrijke groep tijdens mijn promotie. Ik had vooral veel contact met de mensen uit Jans groep. Natalie, we hebben vaak goede discussies gehad over experimenten. We konden samen ook fijn mopperen op alles wat er volgens ons niet klopte om lekker af te reageren. Bach, you were a great guy to work with. You practically lived in the lab and no experiment was ever too big to perform for you. Alex, we worked a lot together as our projects relied on using many of the same experimental pipelines. You have developed yourself very well into a valuable asset of the cBite team with your bioinformatics skills. Nick, je begon bij mij als master student en door je goede fit in het team begon je niet snel daarna op een project dat sterk gerelateerd is aan dat van mij. Ik vond het altijd fijn om met je samen te werken, vooral toen ik op het einde van mijn contract (officieel) het lab niet meer op mocht en je me hiermee uit de brand hielp voor de laatste foto's. Ik vond het gezellig om je bij ons op kantoor te krijgen na je adoptie door BST. Andere mensen die ik wil bedanken zijn: Marloes en Christien.

Buiten cBite wil ik Anouk bedanken voor de steun in het lab, vooral in het begin. Tom, ik heb altijd fijn met je samengewerkt zowel toen je bij Materiomics werkte als wanneer je overging naar TR. Ik moet nog altijd lachen als ik terugdenk hoe je het graag wilde dat ik er even naast ging zitten wanneer je een bepaald probleem wilde oplossen met een microscoop omdat je de indruk had dat microscopen al beter werkten als ik er naast zat. Andere mensen uit deze grote voormalig TR groep die ik wil bedanken (en waarvan ik bang 
ben dat ik er waarschijnlijke enkele hier vergeet te vermelden) zijn: Erik, Bjorn, Ana, Eelco, Anne, Ziryan, Ivan, Angad, Paul, Mijke, Andrea, Giulia, Wim, Tim, Vanessa, Corina, David, Wim, Hugo, Lorenzo, Pamela, Roman, en Honglin.

Ik heb veel samengewerkt met enkele mensen van Materiomics. Zonder hen zou deze thesis een stuk minder mooi zijn geworden. Bernke, je was erg belangrijk voor de totstandkoming van deze thesis, vooral in de beginfase, omdat jij eigenlijk de enige was zelf had gewerkt met de Topochip. Ik vind het leuk dat dat je ook deel uitmaakt van mijn promotiecommissie. Yiping, your arrival at Materiomics improved the fabrication process of the Topochips significantly. Your expertise has been invaluable, especially for the development of the Nano-TopoChip. Ook wil ik Natalie, Marloes, James, Niloofar en Joris bedanken.

De goede instructies en hulp van cleanroom personeel zorgden ervoor dat een bioloog in staat was om met een beperkt protocol de microfabricatie technieken te kunnen gebruiken om Topochips te maken. Hiervoor wil ik met name Huib, René, Ton en Hans bedanken. Ook wil ik Mark bedanken voor alle SEM sessies die op het einde nodig waren om de laatste fabricatieproblemen met de Nano-Topochip op te lossen en de mooie foto's die het uiteindelijk heeft opgeleverd.

Bij de Technology assessment aan het einde van dit proefschrift heb ik veel hulp gehad van Verena stimberg. Heel erg bedankt hier voor.

Ons kantoor ZH248 was voor mij de meest bezochte plek tijdens mijn promotie en voelde voor mij dan ook als een tweede huis. Erwin, Bas, Nick en Mike waren gezellige kantoorgenoten en we hebben samen veel gelachen. Omdat ik de automatenkoffie niet kon verteren hadden we de koffie en thee zelf goed geregeld. Een goed muziekje (Als Bas niet te enthousiast werd met zijn soms wat uiteenlopende muzieksmaak) hielp voor zowel de sfeer als de productiviteit. Ik denk dat de goede sfeer op het kantoor één van de redenen was dat we samen een aantal leuke dingen hebben georganiseerd zoals enkele BST groepsuitjes en een editie van de MIRA triatlon.

Mijn paranimfen zijn dan ook de heren warmee ik het langst op kantoor heb gezeten, Erwin en Bas. Beide hebben mij enthousiast gemaakt voor het genieten van lekkere whisky's, iets wat we samen moeten blijven doen. Daarnaast delen we interesses in de flauwe humor en de mooie auto's van Top Gear. Ook speelden we af samen oude computerspelletjes zoals Red Alert voor de ultieme gevoelens van nostalgie. Erwin, toen ik net begon konden we al snel goed met elkaar opschieten. Je betrok me bij jouw activiteiten zoals fitness en bierdrinken met de jongens van 92. Ik heb veel van je geleerd en zal je hiervoor altijd dankbaar zij. Ik hoop dat het je goed zal gaan samen met Jobke en Esmé. Bas, we hebben altijd goede gesprekken en kunnen samen goed dingen organiseren. Je bent denk ik een van de weinige mensen waarvan ik kan zeggen dat hij nog eigenwijzer is dan ikzelf en dat vind ik mooi. Je laat je niet van de wijs brengen, als ik je wilde muzieksmaak of je favoriete voetbalteam afkraak. Sinds kort heeft je leven een nieuwe positieve wending gekregen door je relatie met Magda en ik wens jullie veel geluk samen.

Een belangrijke factor naast mijn promotie was mijn tijd bij Korbalclub E.K.C. Nääs. Ik heb altijd met veel plezier in de verschillende teams gespeeld. Sport is voor mij een belangrijke vitlaatklep voor mijn emoties, die ik normaal niet makkelijk uit. Soms heeft dit wel eens gevolg dat ik tijdens het sporten wat te heftig reageer op mijn teamgenoten die het hierdoor soms iets te hard te verduren krijgen (vooral tijdens de meer stressvolle periodes van mijn promotie). Ik wil jullie bedanken voor jullie begrip. Naast de sportieve aspecten van de vereniging neem ik graag deel aan de meer gezellige aspecten zoals uitjes met het team en de vaste gezelligheid van de donderdagavonden met de Willingenboys. Hier wil ik Ties gelijk even 
bedanken voor de hulp bij het vormgeven van dit boekje. Met het bestuur hebben we het ook meestal gezellig. We hebben een samen heftige periode gehad tijden het mislukte fusieproces, waar ik toentertijd toch een paar nachten slecht van heb kunnen slapen. Ik ben zeer trots op hoe de vereniging er uiteindelijk uit is gekomen en hoe mooi we de boel nu voor elkaar hebben.

Ik wil mijn familie bedanken voor de steun en interesse tijdens mijn promotie. Vooral Rob, vanaf het begin liet je al blijken dat je erg graag bij mijn promotie wilde zijn. Tot mijn schrik ging dat plan in maart bijna mis en ik ben dan ook zeer blij dat je er bij kan zijn.

Iris, je bent een topzus en ik been heel trots op wat je allemaal bereikt heb de afgelopen 5 jaar. Ik wens je veel geluk samen met Jeroen. Pap, bedankt voor het altijd klaarstaan voor allerlei meer praktische zaken zoals verhuizingen en doe het zelf klussen. Ik vind het altijd gezellig om samen met je te werken aan allerlei klusprojecten. Ook Ati, Irene, Reinier en Aliki bedankt.

Mam, bedankt voor het altijd klaar staan op moeilijke momenten. De afgelopen 4 jaar waren voor jouw niet makkelijk. De ziekte en het overlijden van Fred waren zwar voor veel mensen maar vooral voor jouw. Je mist hem nog elke dag en ik moet toegeven dat op het moment dat ik dit schrijf dat ik hem nu ook weer mis, vooral als ik bedenk dat hij de gene was mij meenam naar de presentatie die er toe leidde dat ik uiteindelijk deze promotie heb gedaan! Ik hoop dat je het geluk weer gaat vinden.

Lieve Taiga, bedankt voor je liefde en interesse voor alles wat ik doe. Sinds ik met je samen ben heb je een nieuwe dimensie toegevoegd aan mijn leven. Te amo. 


\section{List of publications}

Unadkat HV, Rewagad RR, Hulsman M, Hulshof GFB, Truckenmüller RK, Stamatialis DF, Reinders MJ, Eijkel JC, van den Berg A, van Blitterswijk CA, de Boer J. A modular versatile chip carrier for high-throughput screening of cell-biomaterial interactions. J R Soc Interface. 2013 Jan 6;10(78):20120753.

Hulshof GFB, Liu E, Negro A, Gobaa S, Lutolf MP, Moghe PV, Fernandes H. High-content imaging. Materiomics, Cambridge University Press, 2013, ISBN 978-1-107-01677-4

Hulsman M, Hulshof GFB, Unadkat H, Papenburg BJ, Stamatialis DF, Truckenmüller R, van Blitterswijk C, de Boer J, Reinders MJ. Analysis of high-throughput screening reveals the effect of surface topographies on cellular morphology. Acta Biomater. 2015 Mar;15:29-38.

Reimer A, Vasilevich A, Hulshof GFB, Viswanathan P, van Blitterswijk CA, de Boer j, Watt FM. Scalable topographies to support proliferation and Oct4 expression of human induced pluripotent stem cells. Scientific reports. 2016; 6:18948

Hulshof GFB, Hulsman M, Vasilevic A, Zhao Y, Levers M, Fekete N, Papenburg B, de Boer M, Yuan H, Signh S, Bray M, Logan D, Reinders M, Carpenter A, van Blitterswijk C, Stamatialis D, de Boer J. Mining for osteogenic surface topographies: In silico design to in vivo osseo-integration (In preparation)

Hulshof GFB, Mihajlovich M, de Boer J, Masereeuw R, Stamatialis D. New insights into the effects of biomaterial chemistry and topography on morphology and function of kidney epithelial cells (In preparation)

Hulshof GFB, Zhao Y, Vasilevic A, Papenburg B Stamatialis D, de Boer J. The Nano-TopoChip: High-throughput screening platform for nanotopographies. (In preparation) 


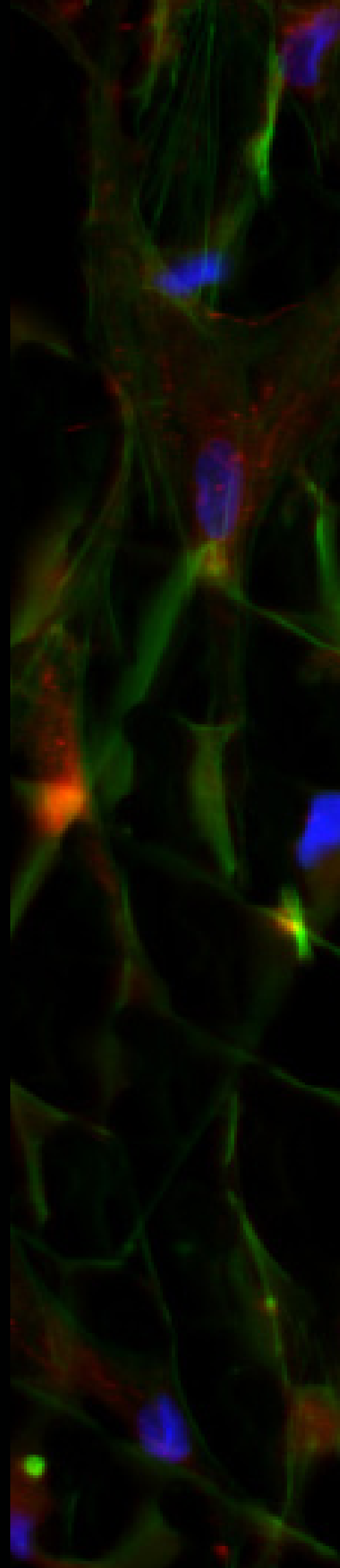

